LA-7792-NS

Informal Report

UC-32

Issued: April 1979

\title{
A Relativity Primer for Particle Transport A LASL Monograph
}

\author{
C. J. Everett \\ E. D. Cashwell
}

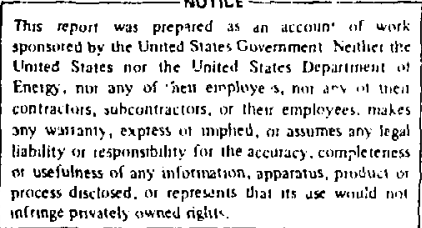




\section{PREFACE}

An attempt is made to present the principles of special relativity, insofar as it applies to systems of particles, in a form which clarifies their possible transmutations, and makes explicit the computational methods of dealing with such phenomena, from the viewpoint of Monte Carlo particle transport. Some mathematical side-lights are included which are logicaily valid; however, any resemblance to physics is purely coincidental.

We are greatly indebted to Leah Peterson for typing the long and difficult manuscript, and to Shirley Cashwe11. for providing the many excellent figures. 
CONTENTS

Chapter I. PARTICLE DYNAMICS

1. The parameters of a particle

2. A relativistic gas

3. Charged particle ballistics

4. Motion against "friction"

5. The Lorentz transformation

6. Time dilatation

7. Fitzgerald contraction

8. The velocity transformation

9. The direction transformation

10. Transformation of the energy 4-vector $(c P, E)$

11. The force transformation

12. Energy transformation and Doppler effect

13. The momentum ellipsoid

Chapter II. CLASSES DF SYSTEMS.

14. Systems of particles

15. The class of a system

16. The two kinds of classes

17. The $\Sigma^{\prime}$ frame of a class

18. Systems of zero total momentum

19. The main existence theorem

20. Systems of two particles

21. Systems of many particles

Chapter III. TRANSMUTATIONS OF SYSTEMS

22. Transmutations

23. The Q-value

24. Decay

25. Decay into two particles

26. Decay into three particles

27. Collisions with target at rest (TAR)

28. Fusion (TAR, $I=1$ ) 
Chapter III. (continued)

29. Two particle product systems (TAR, $I=2$ )

30. Elastic collision (TAR, $I=2$ )

31. Elastic collision (TAR, $I=2, e_{i}=0$, Compton)

32. Elástic co:lision (TAR, $I=2, e_{1}=0$, polarized case)

33. Pair production (TAR, I=3)

34. Collisions with target in motion

35. Colliding beams

Chapter IV. CROSS SECTIONS

36. Mean free path in a gas

37. Transformation of differential cross sections

\section{APPENDICES}

I. COORDINATES AND ROTATIONS

II. A STANDARD DEVICE FOR SCATTERING

III. A GENERAL LORENTZ TRANSFORMATION

\section{TABLES}

I. SOME PHYSICAL CONSTANTS

II. SOME ASTRONOMICAL CONSTANTS

III , SOME "FUNDAMENTAL" PARTICI.ES

IV. SOME NEUTRAL ATOM REST MASSES

V. SOME FORMULAS 


\begin{abstract}
The basic principles of special relativity involved in Monte Carlo transport problems are developed with emphasis on the possible transmutations of particles, and on computational methods. Charged particle ballistics and polarized scattering are included, as well as a discussion of colliding beams.
\end{abstract}


Chapter 1

PARTICLE DYNAMICS

1. The parameters of a particle. In an inertiai frame $\Sigma$ of events $(R, t)$, a definite type of particle is assigned a constant proper rass $\mathrm{m} \geqq 0$. For a matcrial particle (electron, meson, nucleon, . . .) $\mathrm{m}$ is positive, being the rest mass, or mass of the particle at rest in $\Sigma$, whereas an immaterial particle (photon, neutrino,... .) has a proper $\operatorname{mass} m=0$.

A particle moving on a trajectory $(R(t), t)$ has velocity $V=\dot{R}$, and speed $v=|v| \geqq 0$, its direction $\because$ (if $v>0$ ) being determined by the relation $V=v \psi$, where $|\psi|=1$. If $\mathrm{ds} / \mathrm{dt}>0$ for the arc length $\mathrm{s}$, it follows from the identity

$$
v^{\Psi}=V=\frac{\mathrm{dR}}{\mathrm{ds}} \frac{\mathrm{d} s}{\mathrm{dt}}
$$

that $v=\mathrm{ds} / \mathrm{dt}$ and $\psi=\mathrm{dR} / \mathrm{ds}$, the dircction of the trajectory tangent.

If $\mathrm{m}=0$, the speed $\mathrm{v}$ has the constant value $\mathrm{c} \cong 3 \times 10^{10} \mathrm{~cm} / \mathrm{sec}$, whereas $0 \leq v<c$ if $m>0$. It is customary to write $\beta=v / c \leq 1$, and also, for $m>0$,

$$
\gamma=1 /\left(1-\beta^{2}\right)^{\frac{1}{2}} \geq 1
$$

Some relations frequently used are

$$
\gamma^{-2}+\beta^{2}=1, \quad \beta=\left(1-\gamma^{-2}\right)^{\frac{1}{2}}, \quad \gamma \beta=\left(\gamma^{2}-1\right)^{\frac{1}{2}}, \quad \gamma(1-\beta) \cdot \gamma(1+\beta)=1
$$

At time $t$, a particle has a positive mass $M$. For a material particle, $M$ is speed dependent, being determined by 


$$
M=m Y \geq m>0
$$

However, Li.: hites $M$ of an immaterial particlc is an independent parameter, which may have any positive valua.

The momentum of a particle is the vector

$$
P=M V
$$

of magnitude $p=|P|=M v$. Since $V=v \Psi$, we infer that $P=M v \Psi=p \Psi$, so that $P$ also determines the direction $\Psi$.

Particles of both kinds, with $m>0$, may be treated in a uniform way by virtue of the "validity condition:"

A number $M$ and vector $P$ are possible values of the mass and momentum of a particle of proper mass $m \geq 0$ iff they satisfy the conditions

$$
M>0 \quad 1^{2}=c^{2}\left(M^{2}-m^{2}\right)
$$

(The non-relativistic analogue is $\mathrm{P}^{2}=2 \mathrm{mk}$. )

The energy of a particle is defined as $E=M c^{2}$, its proper energy ("rest energy" if $m>n$ ) being $e=m c^{2} \geq 0$. The excess $k=E$-e of $E$ over $e$ is called the kinetic energy of the particle. Note that $k=E=c p$ if $m=0$, while $k=e(\gamma-1) \geq \frac{1}{2} M v^{2} \geq \frac{1}{2} m v^{2}$ for a naterial particle. In fact, $\frac{1}{2} M v^{2}=$ $\frac{1}{2} E \beta^{2}=\frac{1}{2} e \gamma \beta^{2}$, and $\frac{i}{2} \gamma \beta^{2} \leq \gamma-1$ is easily verified from (1).

The "nechanical" parameters $(P, M, m)$ subject to (3) determine all other parameters of a point particle. So do its energy parameters ( $P, E, e)$, which are more convenient in computation. The latter satisfy the important relation. 


$$
\begin{gathered}
E \beta=c p=\left(E^{2}-e^{2}\right)^{\frac{1}{2}} \quad(m>0) \\
\gamma=E / e, \quad \beta=c p / E, \quad \gamma \beta=c p / e, \quad \gamma-1=k / e \quad(m>0)
\end{gathered}
$$

In Monte Carlo practice, one may employ the parameters $(\Psi, k, e)$ for which we have (for e>0)

$$
\gamma=1+(\mathrm{k} / \mathrm{e}), \quad \beta=\left(1-\gamma^{-2}\right)^{\frac{1}{2}}, \quad E=\mathrm{e} \gamma, \quad \mathrm{cp}=\mathrm{E} \beta, \quad \mathrm{cP}=\mathrm{cp} \Psi, \quad \mathrm{v}=\mathrm{c} \beta
$$

The net force acting on a particle is by definition $F=\dot{P}$, a "free" particle being. cre with $F \equiv 0$, hence with $P, M$ (cf. (3)), and $V=M^{-1} P$ constant on its linear trajectory $R(t)=R_{0}+V_{0} t$.

Differentiating in (3) shows that $(\mathrm{MV}) \cdot \dot{\mathrm{P}} \equiv \mathrm{P} \cdot \dot{\mathrm{P}}=\mathrm{c}^{2} \mathrm{M} \dot{\mathrm{M}}$, and hence we have the power input

$$
\mathrm{F} \cdot \mathrm{V}=\dot{\mathrm{P}} \cdot \mathrm{V}=\mathrm{c}^{2} \dot{\mathrm{M}}=\dot{\mathrm{E}}=\dot{\mathrm{k}}
$$

Moreover, if $F=-\operatorname{grad} \phi(R)$, we also have

$$
F \cdot V=(-\operatorname{grad} \phi) \cdot V=-\dot{\phi}
$$

and the combined relations

$$
\dot{\mathrm{k}}=\mathrm{F} \cdot \mathrm{V}=-\dot{\phi}
$$

showing that $k+\phi$ is constant on the trajectory. The work done by a force $F$ on a particle, between points 0 and 1 of its resulting path, is given by 


$$
W=\int_{0}^{1} F \cdot \Psi d s=\int_{0}^{1} F \cdot V d t \quad(d s / d t>0)
$$

From above, we see that

$$
k_{1}-k_{0}=W=\phi_{0}-\phi_{1}
$$

For example, a positive electron, of charge $q \in s u$, in an electrostüic field

$$
F=-\operatorname{grad} \hat{\phi}(\text { dyne; } \cdot s u=v o l t / \mathrm{cm}) \text {, due to a potential }
$$

$\hat{\phi}(\mathrm{R})($ rolt $=\mathrm{erg} / \mathrm{esu})$, is subject to a force

$$
F={ }_{\varrho} F=-\operatorname{grad}(\hat{q \phi}) \text { dyne }
$$

If the difference in (electrical) potential $\hat{\phi}$ between points 0,1 of its resulting trajectory is 1 Volt $\left(=10^{8} / \mathrm{c}\right.$ esu volt), the corresponding $k . e$. increase is

$$
\mathrm{k}_{1}-\mathrm{k}_{\mathrm{o}}=\hat{\mathrm{q}}_{\mathrm{o}}-\mathrm{q} \hat{\phi}_{1}=\mathrm{q}\left(10^{8} / \mathrm{c}\right)=1.6022 \times 10^{-12} \mathrm{erg}
$$

a unit of energy called the electron-Volt $(\mathrm{eV})$.

$$
1 \mathrm{keV}=10^{3} \mathrm{eV} \quad 1 \mathrm{MeV}=10^{6} \mathrm{eV} \quad 1 \mathrm{BeV}=1 \mathrm{GeV}=10^{9} \mathrm{eV}
$$

Thus the electron rest energy $\mathrm{e}=\mathrm{mc}^{2}$ is $.51100 \mathrm{MeV}$

(See the TABLES for physical constants.) 
Every particle is assigned a frequency $v=E / h$, and (if $v>0$ ) a wavelength $\lambda=h / p$, where $h$ is Planck's constant. Clearly we have

$$
\lambda \nu=E / p=c / B \geqq c
$$

with equality iff $\beta=1, m=0$. Thus $\lambda$ is the ordinary wavelength $(\mathrm{c} / \mathrm{v})$ for a photon, and the "de Broglie" wavelength $h /$ Mv for a material particle. The equation

$$
\lambda(\mathrm{cp})=\mathrm{hc}
$$

yields the simple numerical reition

$$
\lambda(\text { fermi }) \times \operatorname{cp}(\mathrm{BeV})=1.2399
$$

where $c p=\sqrt{E^{2}-e^{2}} \quad(=E$ when $m=0)$.

Thus a $1.24 \mathrm{BeV}$ (k.e.) electron has wavelength $\simeq 1$ fermi $=10^{-13} \mathrm{~cm}$.

The Compton wave length $\lambda_{C}$ of a material particle of rest energy $\mathrm{e}>0$ is an intrinsic parameter, defined as the wavelength of a photon having energy equal to the rest energy of the particle. Such a photon has the wavelength

$$
\lambda_{\mathrm{C}}=\mathrm{c} / \mathrm{v}=\mathrm{hc} / \mathrm{hv}=\mathrm{hc} / \mathrm{e}=\mathrm{h} / \mathrm{mc} \text {. }
$$

Applying (8) to the photon shows that

$$
\lambda_{\mathrm{C}}(\text { fermi }) \times \mathrm{e}(\mathrm{BeV})=1.2399
$$

numerically. From the above value of e for the electron one finds its 
Compton wavelength to be $\lambda_{C}=.02426 \AA$. The relation $\lambda_{C} e=h c=\lambda c p$ shows tha

$$
\begin{aligned}
& \lambda_{\mathrm{C}} / \lambda=\mathrm{cp} / \mathrm{e}=\gamma \beta=\left(\gamma^{2}-1\right)^{\frac{1}{2}}, \text { and therefore } \\
& \lambda_{\mathrm{C}} \gtreqless \lambda \quad \text { as } \gamma \gtreqless \sqrt{2} \text { or as } \beta \gtreqless 1 / \sqrt{2} .
\end{aligned}
$$

Thus the wavelength of any material particle becomes less than its Compton wavelength $\lambda_{C}$ when $\gamma=1+k /$ e exceeds $\sqrt{2}$, while its kinetic energy $\mathrm{k}$ becomes greater than its rest energy e when $\gamma$ exceeds 2. For an electron the critical values of $\mathrm{k}$ are $212 \mathrm{keV}$ and $.511 \mathrm{MeV}$ respectively.

Note 1. It is sometimes convenient to replace the time $t$ by the distance parameter $\tau=c t$, with the corresponding dimensionless "velocity"

$$
\bar{V}=d R / d \tau=V / c=\left(\beta_{x}, \beta_{y}, \beta_{z}\right)=\beta \cdot \Psi
$$

in terms of which we have

$$
c P=E \bar{V}
$$

Example 1. A particle of rest energy $e=500 \mathrm{MeV}$ and k.e. $k=800 \mathrm{MeV}$ has

$$
\begin{aligned}
& \gamma=13 / 5, \beta=12 / 13, E=1300 \mathrm{MeV}, \mathrm{cp}=1200 \mathrm{MeV} \\
& \lambda=1.03 \mathrm{f}<\lambda_{\mathrm{C}}=2.48 \mathrm{f}, \mathrm{v}=2.77 \times 10^{10} \mathrm{~cm} / \mathrm{sec}, \text { and } \\
& \mathrm{p}=6.41 \times 10^{-14} \mathrm{gm} \mathrm{cm} / \mathrm{sec} \text {. Note that } \\
& \lambda \nu=3.25 \times 1 \mathrm{c}^{10}>\mathrm{c}, \mathrm{k}=.800>\frac{1}{2} \mathrm{Mv}^{2}=\frac{1}{2} \mathrm{E} \beta^{2}=554>\frac{1}{2} \mathrm{mv}^{2}=\frac{1}{2} \mathrm{e} \beta^{2}=213 \mathrm{M}
\end{aligned}
$$


2. A relativistic gas. Consider a homogeneous isotropic gas of $\mathbf{n}$ particles $/ \mathrm{cm}^{3}$, each of proper mass $m(\supseteq 0)$, of which the fraction $f(k) d k$ have $k$.e. on $(k, k+d k), 0<k<\infty$. The numerical flux of particles, in the indicated ranges of $k$, and spherical coordinates $(\theta, \phi)$ for direction, is seen from Fig. 1 to be

$$
\begin{aligned}
& N(k, \theta, \phi) d k d \theta d \phi= \\
& n(\Delta A \cdot v \Delta t \cos \theta) f(k) d k((\sin \theta d \theta d \phi) / 4 \pi) / \Delta A \Delta t= \\
& (n / 4 \pi) v f(k) d k \sin \theta \cos \theta d \theta d \phi \text { per } \mathrm{cm}^{2} \sec
\end{aligned}
$$

where $v=v(k)$ is the speed as a function of k.e.

Successive integrations, on $0 \leq \phi \leq 2 \pi, 0 \leq \theta \leq \pi / 2$, and $0<k<\infty$, show the various resulting (one-way) fluxes to be

$$
\begin{aligned}
& N(k, \theta) d k d \theta=(n / 2) \vee f(k) d k \sin \theta \cos \theta d \theta \\
& N(k) d k=(n / 4) v f(k) d k \\
& N=(n / 4) \bar{v} \quad \text { per } \mathrm{cm}^{2} \sec
\end{aligned}
$$

while the k.e. flux is

$$
\phi=\int_{0}^{\infty} k N(k) d k=(n / 4) \overline{k v} \quad \mathrm{erg} / \mathrm{cm}^{2} \mathrm{sec}
$$

Regarding the pressure $P$ at a "wall" as the total change of normal component of momentum per sec per $\mathrm{cm}^{2}$ we find from (1) 


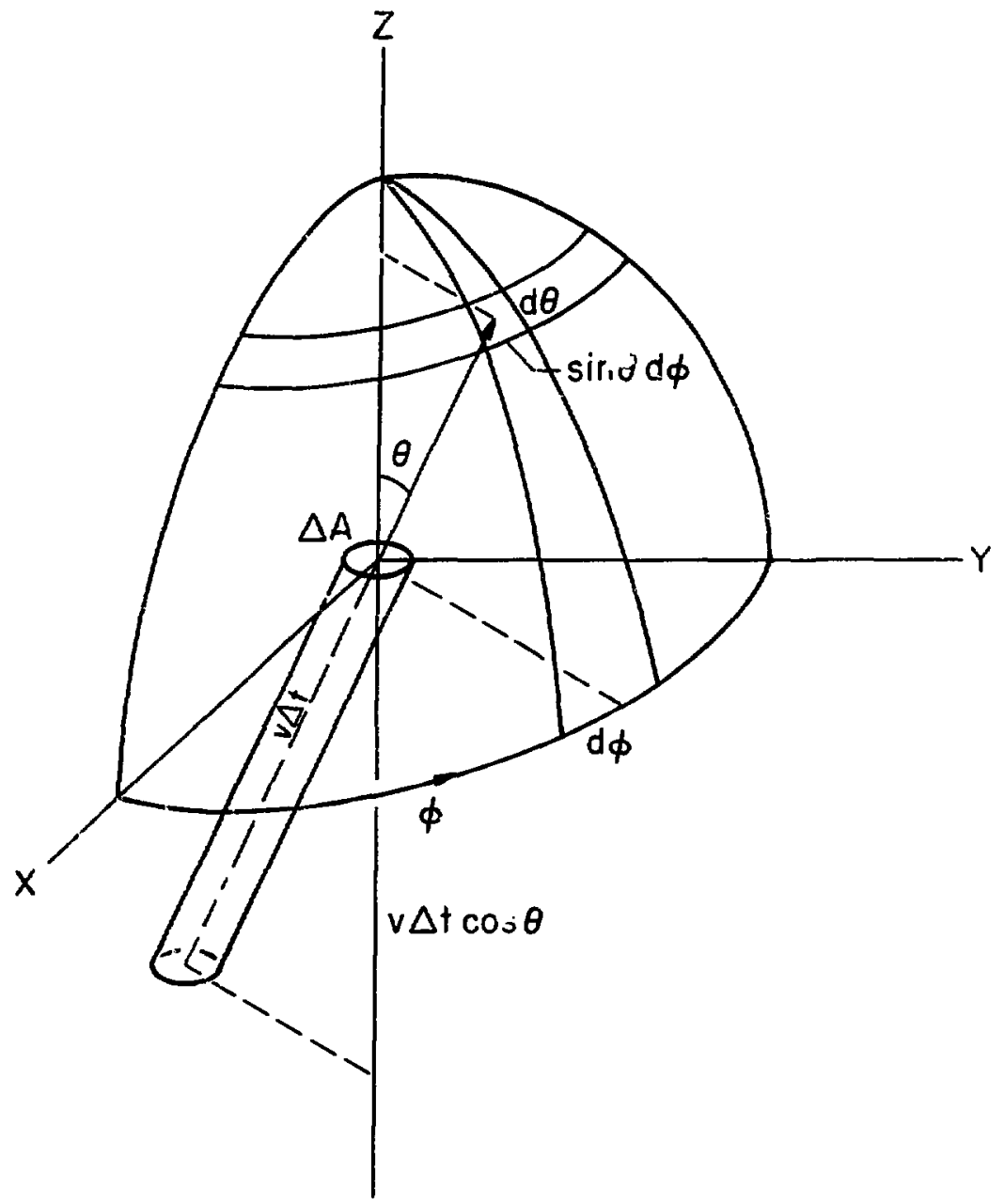

Fig. 1 .

F1ux of a Gas 


$$
P=\int_{6}^{\infty} \int_{0}^{\pi / 2}(2 p \cos \theta) N(k, \theta) d k d \theta=(n / 3) \overline{p v}
$$

in dyne $/ \mathrm{cm}^{2}=\mathrm{erg} / \mathrm{cm}^{3}$, with $\mathrm{p}=\mathrm{p}(\mathrm{k})$.

The k.e. per $\mathrm{cm}^{3}$ on $(k, k+d k)$ is

$$
Y(k) d k=k \cdot n f(k) d k
$$

with the total energy density

$$
K=\int_{0}^{\infty} K(k) d k=n \bar{k} \quad \operatorname{erg} / \mathrm{cm}^{3}
$$

For a gas of photons $(m=0, v=c, k=E=c p=h \nu)$ at "temperature" $\theta=k T$, the function $K(k)$ in (5) is the "Planck density" $(0<k<\infty)$

$$
K(k) d k=8 \pi(h c)^{-3} k^{3} d k /\left(e^{k / \theta}-1\right) \quad \mathrm{erg} / \mathrm{cm}^{3}
$$

With this as a starting point, we infer from (6) that

$$
n f(k) d k=8 \pi(h c)^{-3} k^{2} d k /\left(e^{k / \Theta}-1\right)
$$

and upon integration (Note 1) fi.ld that

$$
\mathrm{n}=15 \pi \zeta(3)(\mathrm{hc})^{-3} \Theta^{3} \quad \text { photons } / \mathrm{cns}^{3}
$$

is the (temperature dependent!) numerical density. Hence from (9), the probability of $k$ on $(k, k+d k)$ is

$$
f(k) d k=\left(2 \zeta(3) \Theta^{3}\right)^{-1} k^{2} d k /\left(e^{k / \theta}-1\right)
$$


On the other hand, integration of (8) shows the total energy density to be

$$
K=(8 / 15) \pi^{5}(h c)^{-3} \Theta^{4}=n \bar{k}
$$

so from (10) and (12) the average photon energy is

$$
\bar{k}=k / n=\pi^{4} \Theta / 30 \zeta(3) \quad \text { erg }
$$

Evaluation of (3), (4), (5) is now trivial, since $v(k) \equiv c$, and $p v=c p=k$. Thus, the nunerical flux is

$$
N=(n / 4) c=4 \pi \zeta(3) c(h c)^{-3} \theta^{3} \quad \text { photons } / \mathrm{cm}^{2} \mathrm{sec}
$$

carrying an energy

$$
\phi=(n / 4) c \bar{k}=(c / 4) K=(2 / 15) \pi^{5} c(h c)^{-3} \theta^{4} \equiv \sigma T^{4}
$$

$\mathrm{erg} / \mathrm{cm}^{2}$ sec, where $\sigma$ is the "Stefan-Boltzmann" constant. Finally, we have for the radiation pressure

$$
P=(\mathrm{n} / 3) \bar{k}=\mathrm{K} / 3 \quad \mathrm{erg} / \mathrm{cm}^{3}
$$

where $\mathrm{K}$ is given by (12).

Note 1. The values given for $n$ and $k$ may be verified from tha formula

$$
I(s)=\int_{0}^{\infty} x^{s-1} d x /\left(e^{x}-1\right)=\Gamma(s) \zeta(s), \quad s>1,
$$


where $\Gamma(s) \equiv \int_{0}^{\infty} x^{s-1} e^{-x} d x(s>0)$ is the $\Gamma$-function, with values $\Gamma(s)=(s-1) !$, $\mathrm{s}=1,2,3, \ldots,(0 ! \equiv 0)$, and $\zeta(\mathrm{s})=\Sigma_{\mathrm{m}=1}^{\infty} \mathrm{m}^{-\mathrm{s}}(\mathrm{s}>1)$ is the Riemann $\zeta$-function.

One knows $\zeta(3)=1.2021$ and $\zeta(4)=\pi^{4} / 90$. The formula (17) results from termwise integration using the geometric series

$$
e^{-x} /\left(1-e^{-x}\right)=\sum_{m=1}^{\infty} e^{-m x}, x>0
$$

Note 2. For a relatjvistic material gas, the functions $v=v(k), p=p(k)$ may be obtiined explicitly from the relations

$$
\begin{array}{ll}
\gamma=1+k / c & \beta=\left(1-\gamma^{-2}\right)^{\frac{1}{2}} \\
v=c \beta & c p=\gamma \beta e
\end{array}
$$

Note 3. For comparison, we list below the main results for the Planck photon gas and the non-relativistic material Maxwell gas. The averages for the latter are easily derived from the Maxwell energy distribution, the relations $v=(2 \mathrm{k} / \mathrm{m})^{\frac{1}{2}}, p v=2 \mathrm{k}$, and the properties of the $\Gamma$-function (Table $v$ ). 


$$
\begin{aligned}
& n=16 \pi \zeta(3)(h c)^{-3} o^{3}, \quad n=0 \quad n, m>0 \text { given constants } \\
& f(k) d k=\left(2 \zeta(3) 0^{3}\right)^{-1} k^{2} d k /\left(c^{k / 0}-1\right) \quad f(k) d k=\frac{2}{\sqrt{3}}()^{-3 / 2} k^{1 / 2} e^{-k / \theta} d k \\
& \bar{v}=c \quad \bar{v}=2(2 / \pi m)^{\frac{1}{2}} 0^{\frac{1}{2}} \\
& \bar{k}=\pi^{4} 0 / 30 \zeta(3) \quad \bar{k}=3 / 2 \theta \\
& \overrightarrow{\mathrm{k}} \overrightarrow{\mathrm{v}}=\mathrm{c} \overline{\mathrm{k}} \\
& \overline{\mathrm{kv}}=4(2 / \pi \mathrm{m})^{1 / 2} \theta^{3 / 2} \\
& \overrightarrow{p v}=\bar{k} \\
& \overrightarrow{p V}=2 \vec{k}=30 \\
& k=n \bar{k}=(8 / 15) \pi^{5}(\text { hc })^{-3} 0^{4} \\
& \mathrm{k}=\mathrm{n} \overline{\mathrm{k}}=\frac{3}{2} \mathrm{n} \theta \\
& \mathrm{N}=\frac{\mathrm{n}}{4} \overline{\mathrm{v}}=\frac{\mathrm{c}}{4} \mathrm{n} \\
& \mathrm{N}=\frac{n_{1}}{4} \overline{\mathrm{v}}=(\mathrm{n} / 2)(2 / \pi \mathrm{m})^{\frac{1}{2}} \theta^{\frac{1}{2}} \\
& \phi=\frac{n}{4} \overline{k v}=\frac{c}{4} K \\
& \phi=\frac{n}{4} \overline{k v}=n(2 / \pi m)^{1 / 2} \Theta^{3 / 2} \\
& P=\frac{n}{3} \overrightarrow{p v}=k / 3 \\
& P=\frac{n}{3} \overline{p V}=n \Theta=\frac{2}{3} \mathrm{~K}
\end{aligned}
$$

Note 4. The Maxwe11 densi-y $\mathrm{f}(\mathrm{k})$ of Note 3 may be deduced from two nypotheses concerning the velocity distribution, and the perfect gas equation of state $P V=R T$. Let $P\left(v_{x}, v_{y}, v_{z}\right)$ he the probability density for the velocity $V=\left(v_{x}, v_{y}, v_{z}\right)$. From the isotropy assumption

$$
\text { I. } \quad P\left(v_{x}, v_{y}, v_{z}\right)=F(v) ; \quad v=|v|
$$

follows the identity of the marginal densities

$$
p_{x}(w) \equiv p_{y}(w) \equiv p_{z}(w) \equiv p(w)
$$


From this and the independence hypothesis

$$
\text { II. } \quad P\left(v_{x}, v_{y}, v_{z}\right)=p_{x}\left(v_{x}\right) \cdot p_{y}\left(v_{y}\right) \cdot p_{z}\left(v_{z}\right)
$$

we obtain the equations

$$
\begin{aligned}
& p\left(v_{x}\right) \cdot p\left(v_{y}\right) \cdot p\left(v_{z}\right) \equiv F(v) \\
& v_{x}^{2}+v_{y}^{2}+v_{z}^{2} \equiv v^{2}
\end{aligned}
$$

For an arbitrary curve $V=V(s)$, through the point $\left(v_{x}, v_{y}, v_{z}\right)$, and 1:ing on the sphere $V \equiv v$, we have then

$$
\begin{aligned}
& \frac{p^{\prime}\left(v_{x}\right) v_{x}^{\prime}}{p\left(v_{x}\right)}+\frac{p^{\prime}\left(v_{y}\right) v^{\prime} y}{p\left(v_{y}\right)}+\frac{p^{\prime}\left(v_{z}\right) v_{z}^{\prime}}{p\left(v_{z}\right)} \equiv 0 \\
& v_{x} v_{x}^{\prime}+v_{y} v_{y}^{\prime}+v_{z} v_{z}^{\prime} \equiv 0
\end{aligned}
$$

It follows that

$$
\left(p^{\prime}\left(v_{x}\right) / p\left(v_{x}\right), p^{\prime}\left(v_{y}\right) / p\left(v_{y}\right), p^{\prime}\left(v_{z}\right) / p\left(v_{z}\right)\right) \equiv\left(v_{x}, v_{y}, v_{z}\right) \cdot K
$$

where $K$ is a constant. Hence

$$
p^{\prime}(w) / p(w)=-w / \sigma^{2}, \quad \sigma>0 \text { constant }
$$

and

$$
p(w)=A e^{-w^{2} / 2 \sigma^{2}}
$$

where

$$
A=\left(2 \pi \sigma^{2}\right)^{-\frac{1}{2}} \text { is determined from }
$$




$$
1=\int_{-\infty}^{\infty} p(w) d w
$$

Hence $P\left(v_{x}, v_{y}, v_{z}\right) d v_{x} d v_{y} d v_{z}$

$$
=A^{3} e^{-v^{2} / 2 \sigma^{2}} d v_{x} d v_{y} d v_{z} \equiv Q(v, \theta, \phi) d v d \theta d \phi
$$

where the latter is the density for speed and direction in spherical coordinates. This yields

$$
Q(v, \theta, \phi) d v d \theta d \phi=A^{3} e^{-v^{2} / 2 \sigma^{2}}\left(v^{2} \sin \theta\right) d \theta d \phi d v
$$

whence

$$
q(v) d v=4 \pi A^{3} v^{2} e^{-v^{2} / 2 \sigma^{2}} d v
$$

gives the marginal speed density. From $f(k) d k=q(v) d v$, where $k=\frac{1}{2} m v^{2}$, then follows

$$
f(k) d k=(2 / \sqrt{\pi})\left(\sigma^{2} m\right)^{-3 / 2} k^{1 / 2} e^{-k / \sigma^{2} m} d k
$$

The mean of $\mathrm{pv}=m v^{2}=2 \mathrm{k}$ is therefore

$$
\overline{p v}=2 \int_{0}^{\infty} k f(k) d k=3 \sigma^{2} m
$$

This serves to evaluate the constant $\sigma^{2}$ by appeal to the equation $P V=R T=$ $N_{0} K T=N_{2} \Theta, \quad$ or

$$
P=n \Theta
$$

For, we know from (5) that 
$-15-$

$$
P=\left(\frac{\mathrm{n}}{3}\right) \overline{\mathrm{pv}}
$$

and hence

$$
n \Theta=P=\frac{n}{3} \overline{p v}=\left(\frac{n}{3}\right)\left(3 \sigma^{2} m\right)=n\left(\sigma^{2} m\right) .
$$

Thus $\sigma^{2} \mathrm{~m}=\theta$, and we therefore have

$$
f(k) d k=(2 / \sqrt{\pi}) \theta^{-3 / 2} k^{1 / 2} e^{-k / \theta} d k
$$


3. Charged particle ballistics. In this section, we derive the trajectory of a non-radiating charged particle in the presence of constant uniform fields of the following types: (I) electrostatic $E$, (II) magnetic $H$, (III) $E$ and $H$ superimposed in orientations which are (A) paralle1, (B) perpendicular (with $|H| \gtreqless|E|)$, (C) arbitrary non-parallel and non-perpendicular.

The independent variable $t$ (sec) will be replaced by the distance parameter $\tau=c t$, and the velocity $V$ by the dimensionless vector

$$
\bar{V}=d R / d t=V / c=\left(\beta_{x}, \beta_{y}, \beta_{z}\right)=\beta \Psi,
$$

with $|\bar{v}|=\beta$. We also transform $\tau$ to the variable $\lambda$ via the monotone increasing function

$$
\lambda=\lambda(\tau)=\int_{0}^{\tau} \mathrm{d} \tau / \gamma(\tau)
$$

which implies that $d \tau / d \lambda=\gamma \geq 1$. Adopting the component notation $\mathrm{dR} / \mathrm{d} \lambda=(\mathrm{X}, \mathrm{Y}, \mathrm{Z})$, we then have

$$
(X, Y, Z)=d R / d \lambda=\frac{d R}{d \tau} \frac{d \tau}{d \lambda}=\gamma \bar{V}=\gamma \beta \Psi,
$$

and rence

$$
X^{2}+Y^{2}+z^{2}=\gamma^{2} \beta^{2}=\gamma^{2}-1
$$

These conventions enable us to write the nomentum as

$$
P=m \gamma(d R / d t)=\frac{e}{c} \frac{d R}{d \tau} \cdot \frac{d \tau}{d \lambda}=\frac{e}{c}(d R / d \lambda)
$$


and its time derivative

$$
\dot{\mathrm{p}}=\mathrm{e} \cdot \frac{\mathrm{d}}{\mathrm{d \tau}}\left(\frac{\mathrm{dR}}{\mathrm{d} \lambda} ;=\mathrm{er}^{-1}\left(\frac{\mathrm{d}^{2} \mathrm{R}}{\mathrm{d} \lambda^{2}}\right)\right.
$$

The force law $\dot{\mathrm{p}}=\mathrm{F}$ then assumes the form

$$
d^{2} R / d \lambda^{2}=e^{-1} \gamma F
$$

Thus the equations of motion may be written as

$$
\frac{d}{d \lambda}(X, Y, Z)=e^{-1} \gamma F
$$

where

$$
\frac{d}{d} \lambda(x, y, z)=(X, Y, Z)
$$

The basic relations for the cgs units employed are indicated by the schematic equations

$$
Q E=F=Q(\bar{V} \times H)
$$

$Q$ charge $(<0)$ in esu $(Q= \pm n q)$

$E$ electrostatic field (dyne $/$ esu $=v 01 t / c m$ )

$H$ magnetic field (gauss)

$Q E$ electrostatic force (dyne)

$Q(\bar{V} \times H)$ Lorentz force (dyne) 
Since $\bar{V}$ is dimensionless, $E$ and $H$ have the same units. We also define the constants

$$
\begin{aligned}
\mathrm{E}_{1}=|E| \cdots & H=|H| \\
\varepsilon=\mathrm{QE}_{1} / \mathrm{e} & \mu=\mathrm{QH} / \mathrm{e} \quad\left(\mathrm{cm}^{-1}\right)
\end{aligned}
$$

where $e=m c^{2}$ (erg) is the rest energy of the particle.

In computation, $\varepsilon$ and $\mu$ may be evaluated in terms of $E_{1}^{\prime}$ in MV (million Volt) per $\mathrm{cm}, \mathrm{H}$ in gauss, and $\mathrm{e}^{\prime}$ in $\mathrm{MeV}$ by observing that

$$
\begin{aligned}
& \varepsilon=Q_{1} / e=(\underline{n q})\left(E_{1}^{\prime} 10^{6}\right)\left(\frac{10^{8}}{c}\right) /\left(e^{\prime} 10^{6}\right)\left(\mathrm{q} \frac{10^{8}}{\mathrm{c}}\right)= \pm \mathrm{nE}_{1} / \mathrm{e}^{\prime} \\
& \mu=\mathrm{QH} / \mathrm{e}=\left(\underline{+\mathrm{nq})} \mathrm{H} /\left(\mathrm{e}^{\prime} 10^{6}\right)\left(\mathrm{q} \frac{10^{8}}{\mathrm{c}}\right)= \pm \frac{\mathrm{nHc}}{\mathrm{e}^{\prime}} 10^{-14} \simeq \pm 3 \mathrm{n} \times 10^{-4}\left(\mathrm{H} / \mathrm{e}^{\prime}\right)\right.
\end{aligned}
$$

We also note here that an equation of form

$$
\begin{gathered}
k-k_{0}=Q_{1}\left(x-x_{0}\right) \\
\text { implies } k-k_{0}=e\left(Q_{1} / e\right)\left(x-x_{0}\right)=e\left(+n E_{1} / e^{\prime}\right)\left(x-x_{0}\right)
\end{gathered}
$$

and hence the numerical relation

$$
k^{\prime}-k_{0}^{\prime}= \pm n E_{1}^{\prime}\left(x-x_{0}\right)
$$


with $k^{\prime}, k_{0}^{\prime}$ in $M e V$ and $E_{1}^{\prime}$ in $M V / c m$.

Our objective is to find the k.e. $k^{\prime}$, direction $\Psi=\left(a_{x}, a_{y}, a_{z}\right)$ and position $R=(x, y, z)$ at time $t$, in terms of their initial values $k_{0}^{\prime}, \Psi_{0}=\left(a_{x}^{o}, a_{y}^{o}, a_{z}^{o}\right)$, and $R_{0}=\left(x_{0}, y_{0}, z_{0}\right)$. Other initial values may be obtained from the relations

$$
\begin{aligned}
& \gamma_{0}=1+\left(k_{0}^{\prime} / e^{\prime}\right), \quad \beta_{0}=\left(1-\gamma_{0}^{-2}\right)^{\frac{1}{2}} \\
& \left(\beta_{x}^{0}, \beta_{y}^{0}, \beta_{z}^{0}\right)=\beta_{0}\left(a_{x}^{0}, a_{y}^{0}, a_{z}^{0}\right) \\
& \left(X_{0}, Y_{0}, Z_{0}\right)=\gamma_{0}\left(\beta_{x}^{0}, \beta_{y}^{0}, \beta_{z}^{0}\right)
\end{aligned}
$$

The final values are obtained by integrating the equations (8) and (9) by various devices. Since

$$
(X, Y, Z)=\gamma B \Psi
$$

the direction $\Psi$ is always obtainable from $(X, Y, Z)$.

I. Motion in electrostatic field $E$. Suppose a particle of charge $Q= \pm n q$, rest energy e and k.e. $k_{0}^{\prime}>0$ starts from $R=R_{0}$ at time $t=0$ in a direction $\Psi_{0}$, and is subject thereafter to a constant electrostatic field $E=\left(E_{1}, 0,0\right), E_{1}>0$. Its trajectory is then determined by the law

$$
\dot{\mathrm{p}}=\mathrm{F}=\left(\mathrm{QE}_{1}, 0,0\right)=-\operatorname{grad} \phi ; \quad \phi=-\mathrm{QE}_{1} \mathrm{X}
$$

In our rstation, this reads $d(X, Y, Z) / d \lambda=e^{-1} \gamma\left(Q E_{1}, 0,0\right)$ 
or

$$
d(X, Y, Z) / d \lambda=(\varepsilon \gamma, 0,0)
$$

Since $d X / d \lambda=\gamma d X / d \tau$, this integrates to

$$
(X, Y, Z)=\left(\varepsilon \tau+X_{0}, Y_{0}, Z_{0}\right)
$$

Moreover, we have

$$
\left(\varepsilon \tau+X_{0}\right)^{2}+Y_{0}^{2}+Z_{o}^{2}=\gamma^{2}-1
$$

which determines

$$
\gamma=\left\{\left(\varepsilon \tau+x_{0}\right)^{2}+w_{0}^{2}\right\}^{\frac{1}{2}}
$$

where

$$
w_{0}^{2}=Y_{0}^{2}+z_{0}^{2}+1=\gamma_{0}^{2}-x_{0}^{2}=\gamma_{0}^{2}\left(1-\left(\beta_{x}^{0}\right)^{2}\right)
$$

Hence we have the explicit relation

$$
\lambda=\int_{0}^{\tau} d \tau / \gamma=\varepsilon^{-1} \ln \left\{\left[\left(\varepsilon \tau+x_{0}\right)+\gamma\right] /\left(x_{0}+\gamma_{0}\right)\right\}
$$

Now from $F=-\operatorname{grad} \phi$ in $(20)$, we infer that

$$
k-k_{0}=\phi_{0}-\phi=Q E_{1}\left(x-x_{0}\right)
$$




$$
\gamma-\gamma_{0}=\varepsilon\left(x-x_{0}\right)
$$

From (28) and (22) we obtain the position

$$
R=R_{0}+\left(\varepsilon^{-1}\left(\gamma-\gamma_{0}\right), Y_{0} \lambda, z_{0} \lambda\right)
$$

where $\gamma$ and $\lambda$ are given by (24) and (26). The energy $k$ is given by (27) in terms of $x$ in (29).

The trajectory (29) is a curve in the plane of $E$ and $\Psi_{0}$, with $y$ and $z$ monotone. For $Q>0, \varepsilon>0, \gamma$ in (24) and hence $x$ in (29) increase without bound in case $x_{0} \geqq 0, a_{x}^{0} \geqslant 0$. However, if $x_{0}<0, a_{x}^{0}<0, \gamma$ and $x$ first decrease to their minimum values at the turning point of the trajectory, where

$$
\begin{aligned}
& x^{*}=\varepsilon \tau^{*}+x_{0}=0=\gamma_{x}^{*} \beta_{x}^{*}, v_{x}^{*}=0 \\
& \gamma^{*}=W_{0}=\gamma_{0}\left(1-\left(\beta_{x}^{o}\right)^{2}\right)^{\frac{1}{2}} \\
& \lambda^{*}=(2 \varepsilon)^{-1} \ln \left\{\left(1-\beta_{x}^{o}\right) /\left(1+\beta_{x}^{o}\right)\right\} \\
& R^{*}=R_{0}+\left(\varepsilon^{-1}\left(\gamma^{*}-\gamma_{0}\right), Y_{0} \lambda^{*}, Z_{0} \lambda^{*}\right)
\end{aligned}
$$

Thereafter, $\gamma$ and $x$ increase without limit. The trajectory is therefore a parabola-1ike curve in the plane of $E$ and $\psi_{0}$. The case $k=0$ (start from rest) is considered in Note 1.

Guide to computation $I$. $\left(E_{1}, 0,0\right)$

Given $R_{0}, \Psi_{0}, k_{0}^{\prime}>0, e^{\prime}, E_{1}^{\prime}, \varepsilon= \pm n E_{1}^{\prime} / e^{\prime}, \tau($ final $)$ 

a. $\gamma_{0}=1+\left(k_{0}^{\prime} / e^{\prime}\right), \quad \beta_{0}=\left(1-\gamma_{0}^{-2}\right)^{\frac{1}{2}}$
b. $\quad A=\gamma_{0} / \varepsilon, \quad T=\tau / A$
c. $\quad\left(B_{x}^{0}, B_{y}^{0}, B_{z}^{0}\right)=\beta_{0}\left(a_{x}^{0}, a_{y}^{0}, a_{z}^{0}\right)$
d. $\quad B=\left\{\left(T+\beta_{x}^{0}\right)^{2}+\left(\beta_{y}^{0}\right)^{2}+\left(\beta_{z}^{0}\right)^{2}\right\}^{\frac{3}{2}}$
e. $\quad\left(a_{x}, a_{y}, a_{z}\right)=\left(T+\beta_{x}^{0}, \beta_{y}^{0}, \beta_{z}^{0}\right) / B$
f. $\quad G=\left\{1+T\left(2 \beta_{x}^{0}+T\right)\right\}^{\frac{1}{2}}\left(=\gamma / \gamma_{0}\right)$
g. $x=x_{0}+A(G-1), k^{\prime}=k_{0}^{\prime}+n E_{1}^{\prime}\left(x-x_{0}\right)$
h. $L=\ln \left\{\left(T ; \beta_{x}^{\circ}+G\right) /\left(1+\beta_{x}^{o}\right)\right\}$
i. $y=y_{0}+A B_{y}^{o} L, \quad z=z_{0}+A B_{z}^{O} L$

Note 1. If the particle starts from rest at $R_{0}=0$, we have the initial conditions $\beta_{0}=0, \gamma_{0}=1, k_{0}^{\prime}=0$, and find at $\tau>0$

$$
\begin{aligned}
& \gamma=\left(\varepsilon^{2} \tau^{2}+1\right)^{\frac{1}{2}} \rightarrow \infty, \quad \Psi=(1,0,0) \\
& x=\varepsilon^{\prime 1}(\gamma-1), \quad y=0, \quad z=0, \quad k^{\prime}= \pm n E_{1}^{\prime} x
\end{aligned}
$$

Elimination of $\varepsilon$ shows that

$$
(x / \tau)^{2}=(\gamma-1) /(\gamma+1)+1, \quad x / t \rightarrow c
$$

Since $(\gamma-1) /(\gamma+1)<\beta^{2}=v^{2} / c^{2}$, this implies

$$
x / t<v \rightarrow c
$$


As an illustration, consider $\mathrm{a}^{+}$particle $\left(\mathrm{e}^{\prime}=140 \mathrm{MeV}\right)$ in a linear accelerator with a potential drop of $5 \mathrm{MN}$ over 10 meters. Then

$$
E_{1}^{\prime}=5 \times 10^{-3} \mathrm{MV} / \mathrm{cm}, \varepsilon=E^{\prime} / \mathrm{e}^{\prime}=3.57 \times 10^{-5} \mathrm{~cm}^{-1}
$$

At the end of the trip, $x=10^{3} \mathrm{~cm}$, and we find from (31; that $\gamma=1.0357$, $k^{\prime}=5 \mathrm{MeV}$. However, (30) shows that the trip would require $t=25 \times 10^{\circ} \mathrm{sec}$ whereas $\pi^{+}$has a mean intrinsic life of only $2.0 \times 10^{-8} \mathrm{sec}$ (Cf. Notes 6.1 , $6.2)$

For orientation, $: \mathrm{e}$ include below the non-relativistic framework underlying the idea of the Bohr magneton. This involves the motion of a charged particle in a central electrostatic field.

Note 2. A particle of mass $m$ with the circular trajectory

$$
R=\left(r_{0} \cos \omega t, r_{0} \sin \omega t, 0\right)
$$

$\left(\mathbf{r}_{0}, \omega>0\right.$ constant) has a speed $v_{0}=|\dot{R}|=\omega r_{0}$, and an acceleration $\ddot{k}=-\omega^{2} R$. It therefore satisfies the Newtonian force law

$$
\ddot{m P}=F=\frac{-K}{r^{2}} \cdot \frac{R}{r}=-\operatorname{grad} \phi ; \phi=-\frac{K}{r}
$$

provided $-m \omega^{2}=-\frac{k}{r_{0}^{3}}$ where $\omega=v_{0} / r_{0}, i . e .$, in case

$$
r_{0} m v_{0}^{2}=K
$$

Its crbital k.e. is therefore

$$
k_{o}=\frac{1}{2} m v_{o}^{2}=\frac{1}{2}\left(K / r_{o}\right)
$$


and its total energy has the constant value

$$
\mathrm{T}_{\mathrm{o}}=\mathrm{k}_{\mathrm{o}}+\phi_{\mathrm{o}}=-\frac{1}{2}\left(\mathrm{~K} / \mathrm{r}_{\mathrm{o}}\right)
$$

For an arbitrary trajectory just permitting escape, from the same radial distance $r_{o}$, one must have a k.e. $k_{e}$ such that $k_{e}+\phi_{0}=k_{\infty}+\phi_{\infty}=$ $0+0=0$, or

$$
k_{e}=-\phi_{0}=K / r_{0}=2 k_{o}
$$

For the corresponding velocities therefore

$$
v_{e}=\sqrt{2} v_{o}=\sqrt{2} \cdot \sqrt{2 \mathrm{k}_{o} / \mathrm{m}}=\sqrt{2} \sqrt{\mathrm{K} / \mathrm{r}_{\mathrm{o}} \mathrm{m}}
$$

In case of an earth-rocket system, we note in passing the approximate escape velocity

$$
v_{\epsilon}=\sqrt{\frac{2 G M m}{r_{o} m}}=\sqrt{\frac{2 G M}{r_{o}} r_{o}}=\sqrt{2 g r_{o}}=\sqrt{2\left(\frac{32.2}{5280}\right)(4000)} \cong 7 \mathrm{mi} / \mathrm{sec}
$$

Note 3. The circular motion of Note 2 , with $K=Z q^{2}$, was the basis for Bohr's 1-electron atom of atomic number $z$. The $r$ biting electron is assumed to satisfy the classical relation

$$
r_{0} m_{0}^{2}=K=Z q^{2}
$$

(which permits any radius $r_{0}$ with the required $v_{0}$ ), and also the restricting condition 


$$
r_{0} m v_{0}=n \hbar, h \equiv h / 2 \pi
$$

where $n$ is an integer $1,2,3, \ldots$

The latter expresses the quantization of angular momentum $\mathrm{r}_{0} \mathrm{mv}_{0}$, or equivalently the de Broglie idea

$$
2 \pi r_{0}=n \lambda=n\left(h / m v_{0}\right)
$$

Thus $h$ becomes a unit of angular momentum.

The two relations together uniquely determine the radius $r_{n}$ and all other parameters for a stipulated integer $n$; thus

$$
\begin{aligned}
& v_{n}=z q^{2} / \mathrm{nh}^{\prime}=2.2 \times 10^{8}(\mathrm{z} / \mathrm{n}) \mathrm{cm} / \mathrm{sec} \\
& r_{n}=\mathrm{n}^{2} \mathrm{~h}^{2} / \mathrm{mzq}^{2}=.53 \times 10^{-8}\left(\mathrm{n}^{2} / \mathrm{z}\right) \mathrm{cm} \\
& \mathrm{T}_{\mathrm{n}}=-\frac{1}{2}\left(\mathrm{zq} / \mathrm{r}_{\mathrm{n}}\right)=-\mathrm{mz} \mathrm{z}^{2} / 2 \mathrm{n}^{2} \mathrm{~h}^{2}=-13.6\left(\mathrm{z}^{2} / \mathrm{n}^{2}\right) \mathrm{eV}
\end{aligned}
$$

For $n=1,7=1$, we obtain the "fine-structure" constant

$$
\beta_{1} \equiv v_{1} / c=q^{2} / \mathrm{hc} \cong 1 / 137
$$

Note 4. (a) A bar magnet of length $L$ and pole strength $\tilde{m}$ (cgs unit = esu charge) at angle $\theta$ with a uniform magnetic field $H$ (gauss) is subject to a torque $2 \mathrm{R} \times \mathrm{F}=2 \mathrm{R} \times \tilde{\mathrm{mH}}$ ( $\mathrm{cm}$ dyne=erg), with a magnitude

$$
|2 R \times \tilde{m} H|=\tilde{L}|H| \sin \theta \quad \operatorname{erg}
$$

as in rig. 1. The quantity Lm (erg/gauss) is called its magnetic moment. Note the simple relations of cgs units revealed by the schematic equation 


$$
Q|E|=Q^{\prime} / r^{2}=F=m^{n} / r^{2}=\tilde{m}|H|
$$

(b) A plane loop of area A carrying a current $i$ (esu/sec), in the same field $H$, experiences a torque of magnitude

$$
(A i / c)|H| \sin \theta \quad \text { erg }
$$

due to the Lorentz force $L$ of the field on the moving charges. By analogy, $\mathrm{Ai} / \mathrm{c}$ (erg/gauss) is called the magnetic moment of the loop. (Note the units of $(\mathrm{Ai} / \mathrm{C})|\mathrm{H}|$ :

$$
\left.\frac{L^{2}(Q / T)}{(L / T)}|H|=\tilde{L}|H|=L \times \text { dyne }=\text { erg }\right)
$$

(c) The orbital electron in Bohr's $H$-atom $(z=1, n=1)$, if regarded as producing a current

$$
i=q\left(v_{1} / 2 \pi r_{1}\right) \quad \text { esuisec }
$$

in a loop of area $A=\pi r_{1}^{2}$, should give rise to a magnetic moment

$$
\begin{aligned}
\mu_{B}=A i / c=q r_{1} v_{1} / 2 c & =q\left(r_{1} m_{1}\right) / 2 m c=q h / 2 m c \\
& =9.274 \times 10^{-21} \mathrm{erg} / \text { gauss }
\end{aligned}
$$

a unit called the Bohr magneton. 
$-27-$
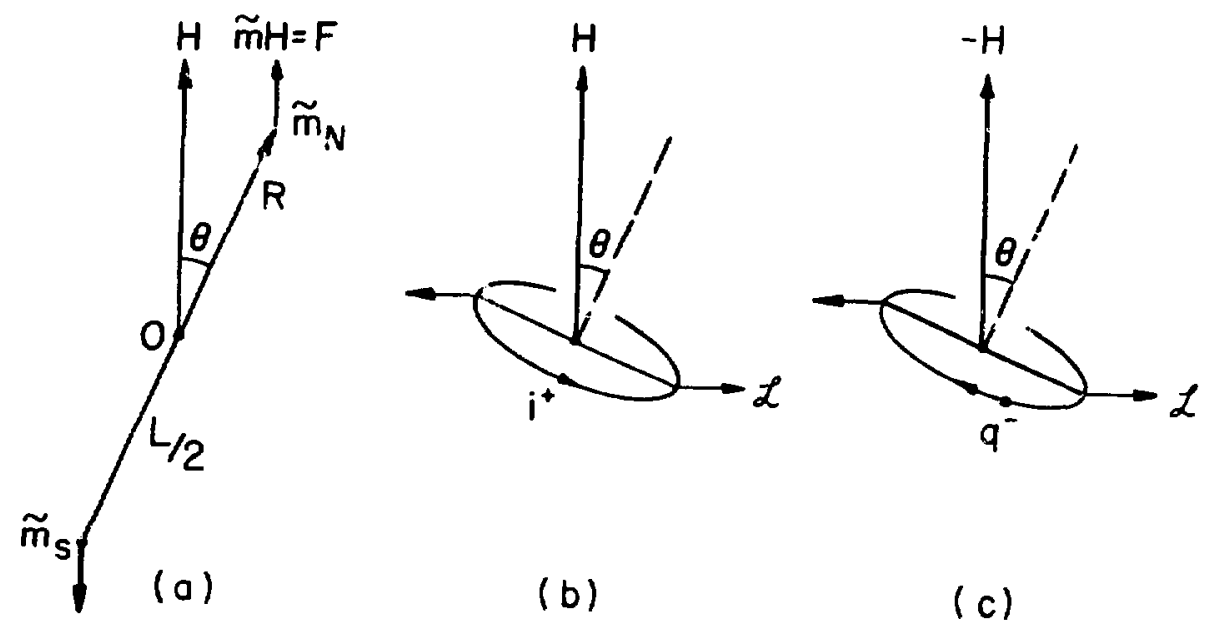

(b)

(c)

Fig. 1 .

Magnetic Moments 
II. Motion in magnetic field $H$. The force equation is now

$$
\dot{P}=F=Q(\bar{V} \times H)
$$

where $F$ is the Lorentz force, for which

$$
\dot{i}=F \cdot V=0
$$

by $(1.6)$, since $V \cdot(\bar{V} \times H)=H(V \times \bar{V})$. Thus $k$ and all other scalar parameters retain their initial values on the resulting trajectory. In particular,

$$
k \equiv k_{0}, \quad \gamma \equiv \gamma_{0}, \quad \beta \equiv \beta_{0},
$$

and hence

$$
\lambda=\int_{0}^{\tau} \mathrm{d} \tau / \gamma \equiv \tau / \gamma_{0}
$$

For a constant magnetic field

$$
H=(-H, 0,0) ; \quad H>0
$$

we may write (32) in cur notation as $d(X, Y, Z) / d \lambda=e^{-1} \gamma Q(\bar{V} \times H)=$ $\mu(X, Y, Z) \times(-1,0,0)$ or

$$
d(X, Y, Z) / d \lambda=(0,-\mu Z, \mu Y)
$$

Thus we have at once

$$
x=x_{0} \quad x=x_{0}+x_{0} \lambda
$$


Moreover, we infer from (36) that

$$
\mathrm{d}^{2}(\mathrm{Y}, \mathrm{Z}) / \mathrm{d} \lambda^{2}=-\mu^{2}(\mathrm{Y}, \mathrm{Z})
$$

and therefore

$$
\begin{aligned}
& Y=Y_{0} \cos \mu \lambda-Z_{0} \sin \mu \lambda \\
& Z=Z_{0} \cos \mu \lambda+Y_{0} \sin \mu \lambda
\end{aligned}
$$

The first constants are obviously necessary, while the second pair is determined by (36), with $\lambda=0$.

Since $(Y, Z)=\mathrm{d}(y, z) / \mathrm{d} \lambda$, integration of (39) gives

$$
F=R_{0}+\left(X_{0} \lambda, \mu^{-1}\left(Z-Z_{0}\right),-\mu^{-1}\left(Y-Y_{0}\right)\right)
$$

Using the initial relations $\left(X_{0}, Y_{0}, z_{0}\right)=\gamma_{0} \beta_{0}\left(a_{x}^{o}, a_{y}^{0}, a_{z}^{o}\right)$ these results can be written in the form

$$
\begin{aligned}
& x=\gamma_{0} \beta_{0}^{a} x \\
& Y=\gamma_{0} \beta_{o}\left(a_{y}^{o} \cos \mu \lambda-a_{z}^{o} \sin \mu \lambda\right) \\
& z=\gamma_{0} \beta_{0}\left(a_{z}^{0} \cos \mu \lambda+a_{y}^{0} \sin \mu \lambda\right) \\
& x=x_{0}+\gamma_{0} \beta_{0}^{a} a_{x}^{\alpha} \lambda \\
& y=\eta_{0}+\mu^{-1} z \quad \eta_{0} \equiv y_{0}-\mu^{-1} \gamma_{0} \beta_{0} a_{z}^{o} \\
& z=\zeta_{0}-\mu^{-1} Y \quad \zeta_{0} \equiv z_{0}+\mu^{-1} \gamma_{0} \beta_{0} a_{y}^{0}
\end{aligned}
$$


where we know $\lambda=\tau / \gamma_{0}$. Since $\gamma, \beta$ are constant, (41) is equivalent to

$$
\begin{aligned}
& a_{x}=a_{x}^{0} \\
& a_{y}=a_{y}^{0} \cos \mu \lambda-a_{z}^{0} \sin \mu \lambda \\
& a_{z}=a_{z}^{0} \cos \mu \lambda+a_{y}^{0} \sin \mu \lambda
\end{aligned}
$$

If $\Psi_{0}=( \pm 1,0,0)$, the particle is unaffected by the field, the trajectory being the line

$$
x=x_{0} \pm v_{0} t \quad y=y_{0} \quad z=z_{0}
$$

parallel to $H$. For $\psi_{0} \neq( \pm 1,0,0)$, we write

$$
\Psi_{0}=\left(a_{x}^{0}, a_{y}^{o}, a_{z}^{o}\right)=\left(\cos \psi_{0}, \sin \psi_{0} \cos \phi_{0}, \sin \psi_{0} \sin \phi_{0}\right)
$$

in terms of spherical coordinates. Relations $(42,43)$ then assumie the more transparent form:

$$
\begin{aligned}
& x=x_{0}+\gamma_{0} \beta_{0}\left(\cos \psi_{0}\right) \lambda \\
& y=\eta_{0}+\mu^{-1} \gamma_{0} \beta_{0} \sin \psi_{0} \sin \left(\phi_{0}+\mu \lambda\right) \\
& z=\zeta_{0}-\mu^{-1} \gamma_{0} \beta_{0} \sin \psi_{0} \cos \left(\phi_{0}+\mu \lambda\right) \\
& a_{x}=\cos \psi_{0} \\
& a_{y}=\sin \psi_{0} \cos \left(\phi_{0}+\lambda \mu\right) \\
& a_{z}=\sin \psi_{0} \sin \left(\phi_{0}+\lambda \mu\right)
\end{aligned}
$$


For $a_{x}^{0}=\cos \psi_{0} \frac{1}{r} 0$, this is a spiral of uniform pitch, with axis parallel to $H$, through the noint $\left(x_{0}, \eta_{0}, \zeta_{0}\right)$, and radius

$$
r_{0}=|\mu|^{-1} \gamma_{0} \xi_{0} \sin \psi_{0}
$$

The time of revolution is given by $\mu \lambda=2 \pi$, namely

$$
t_{0}=2 \pi \gamma_{0} / c|\mu|
$$

In the special case of an initial direction perpendicular to the field $\left(a_{x}^{0}=0, \sin \psi_{0}=1\right)$, the curve reduces to a circle of radius

$$
r_{0}=\gamma_{0} \beta_{0} /|\mu|
$$

in the plane $x=x_{0}$, with center at $\left(x_{0}, n_{0}, \zeta_{0}\right)$. The (cyclotron) period and frequency are then

$$
t_{0}=2 \pi \gamma_{0} / c|\mu| \quad f_{0}=1 / t_{0}=c|\mu| / 2 \pi \gamma_{0}
$$

(Note that $2 \pi r_{0} / t_{0}=\beta_{o} c=v_{o}$, the constant speed.)

We recall here the numerical relation

$$
\mu= \pm 3 n \times 10^{-4}\left(\mathrm{H} / \mathrm{e}^{\prime}\right)
$$

with $\mathrm{H}$ in gauss, $\mathrm{e}^{\prime}$ in $\mathrm{MeV}$. 
Guide to computation I I. $\quad(-\mathrm{H}, 0,0)$

Given $R_{0}, \Psi_{0}, k_{0}^{\prime}, e^{\prime}, \quad \mu= \pm 3 n \times 10^{-4}\left(H / e^{\prime}\right), \tau$
a. $\gamma_{0}=1+\left(k_{0}^{\prime} / e^{\prime}\right), \quad \beta_{0}=\left(1-\gamma_{0}^{-2}\right)^{\frac{1}{2}}, \quad \omega=: 1 / \gamma_{0}$

b. $\quad C=\cos \omega \tau, \quad S=\sin \omega \tau$

c. $\left(a_{x}, a_{y}, a_{z}\right)=\left(a_{x}^{o}, a_{y}^{o} \mathrm{C}-a_{z}^{o} S, a_{z}^{o} \mathrm{C}+a_{y}^{o} S\right)$

d. $x=x_{0}+\beta_{0} a_{x}^{o} t$

$$
\begin{aligned}
& y=y_{o}+w^{-1} B_{o}\left(a_{y}^{o} S-a_{z}^{o}(1-C)\right) \\
& z=z_{0}+w^{-1} B_{o}\left(a_{z}^{o} S+a_{y}^{o}(1-C)\right)
\end{aligned}
$$

e. $k^{\prime}=k_{0}^{\prime}$ 
Note 5. In the case of circular motion $\left(\mathrm{a}_{\mathrm{x}}^{\mathrm{o}}=0\right)$, a $500 \mathrm{MeV} \pi^{+}$paricle $\left(e^{\prime}=140 \mathrm{MeV}\right)$ in a field of $H=16000$ gauss makes a circuit of $r_{0}=i 30 \mathrm{~cm}$ in a time $t_{0}=2.8 \times 10^{-8} \mathrm{sec}$.

In fact,

$$
\begin{aligned}
\mu & =3 \times 10^{-4}\left(\mathrm{H} / \mathrm{e}^{\prime}\right)=.0343 \mathrm{~cm}^{-1} \\
\gamma_{0} & =1+\left(\mathrm{k}^{\prime} / \mathrm{e}^{\prime}\right)=4.57 \\
\gamma_{0} \beta_{0} & =\left(\gamma_{0}^{2}-1\right)^{\frac{1}{2}}=4.46 \\
r_{0} & =\gamma_{0} \beta_{0} /|\mu|=130 \mathrm{~cm} \\
t_{0} & =2 \pi \gamma_{0} / \mathrm{c}|\mu|=2.79 \times 10^{-8} \mathrm{sec} .
\end{aligned}
$$


III. Motion in superimposed fields.

A charged particle in superimposed fields $E, H$ is governed

by the law

$$
\dot{P}=F=Q E+Q \bar{V} \times H
$$

If $Q E=-$ grad $\phi$, then necessarily by $(1.6)$,

$$
\dot{k}=F \cdot V=Q E \cdot V+0=(-\operatorname{grad} \phi) \cdot V=-\dot{\phi}
$$

even though $F$ itself is not derivable from a potential. Hence, for the constant field

$$
E=\left(E_{1}, 0,0\right), \quad E_{1}>0
$$

which we always assume, with

$$
Q E=-\operatorname{grad} \phi, \quad \phi=-\mathrm{QE}_{1} \mathrm{X}
$$

we have throughout Part III the relations

$$
\begin{aligned}
& k-k_{0}=Q E_{1}\left(x-x_{0}\right) \\
& \gamma-\gamma_{0}=E\left(x-x_{0}\right)
\end{aligned}
$$

just as in Part I. In the rest of this section, we consider the possible orientations of $H$ relative to the stipulated field $E$. 


\section{III. (A) Parallel Case.}

We assume

$$
H=(-H, 0,0), H>0
$$

(The case $(H, 0,0)$ is obtained by changing $H$ to $-H$ and $\mu$ to $-\mu$

throughout.) We may now write (50) in our notation as

$$
d(X, Y, Z) / d \lambda=(\varepsilon \gamma,-\mu Z, \mu Y)
$$

Comparing this with (21) and (36), we have as in I and II,

$$
\begin{aligned}
& X=\varepsilon \tau+X_{0} \\
& Y=Y_{0} \cos \mu \lambda-Z_{0} \sin \mu \lambda \\
& Z=Z_{0} \cos \mu \lambda+Y_{0} \sin \mu \lambda \\
& R=R_{0}+\left(\varepsilon^{-1}\left(\gamma-Y_{0}\right), \mu^{-1}\left(Z-Z_{0}\right),-\mu^{-1}\left(Y-Y_{0}\right)\right)
\end{aligned}
$$

Since $\left(X_{0}, Y_{0}, Z_{0}\right)=\gamma_{o} \beta_{o}\left(a_{x}^{0}, a_{y}^{0}, a_{z}^{0}\right)$, we may write (58), (59) in the form

$$
\begin{aligned}
& X=\varepsilon \tau+X_{0} \\
& Y=\gamma_{0} \beta_{0}\left(a_{y}^{0} \cos \mu \lambda-a_{z}^{0} \sin \mu \lambda\right) \\
& Z=\gamma_{0} \beta_{0}\left(a_{z}^{0} \cos \mu \lambda+a_{y}^{o} \sin \mu \lambda\right) \\
& x=x_{0}+\varepsilon^{-1}\left(\gamma-\gamma_{0}\right) \\
& y=\eta_{0}+\mu^{-1} z \quad \eta_{0} \equiv y_{0}-\mu^{-1} \gamma_{0} \beta_{0} a_{z}^{o}
\end{aligned}
$$




$$
z=\zeta_{0}-\mu^{-1} Y \quad \zeta_{0} \equiv z_{0}+\mu^{-1} \gamma_{0} \beta_{0} a_{y}^{0}
$$

Moreover from (58), we see that

$$
\begin{aligned}
& \gamma^{2}-1=X^{2}+Y^{2}+Z^{2}=\left(\varepsilon \tau+X_{0}\right)^{2}+Y_{0}^{2}+Z_{0}^{2}, \text { whence } \\
& \gamma=\left\{\left(\varepsilon \tau+X_{0}\right)^{2}+W_{0}^{2}\right\}^{\frac{1}{2}}, \quad W_{0}^{2}=\gamma_{0}^{2}\left(1-\left(\beta_{X}^{0}\right)^{2}\right) \\
& \lambda=\int_{0}^{\tau} d \tau / \gamma=\varepsilon^{-1} \ln \left[\left[\left(\varepsilon \tau+X_{0}\right)+\gamma\right] /\left(X_{0}+\gamma_{0}\right)\right\}
\end{aligned}
$$

just as in Part $I$, and again we obtain

$$
k=k_{0}+Q E_{1}\left(x-x_{0}\right)
$$

from $x$.

It appears from (61), (62), (63), (64) that the behavior of $x$ and $k$ is precisely that described in Part $I$, while the $y, z$ components of the trajectory are similar to those of Part II but depend on an angle of revolution non-linear in time.

If $\Psi_{0}=(+1,0,0)$, the trajectory is the line

$$
x=x_{0}+\varepsilon^{-1}\left(\gamma-\gamma_{0}\right), \quad y=y_{0}, \quad z=z_{0}
$$

the motion being exactly that in the absence of $H$.

If $\Psi_{0} \not t(+1,0,0)$, then defining spherical coordinates $\psi_{0}, \phi_{0}$ as in (44), we see that 


$$
\begin{aligned}
& x=\varepsilon \tau+x_{0} \\
& Y=\gamma_{0} \beta_{0} \sin \psi_{0} \cos \left(\phi_{0}+\mu \lambda\right) \\
& Z=\gamma_{0} \beta_{0} \sin \psi_{0} \sin \left(\phi_{0}+\mu \lambda\right) \\
& x=x_{0}+\varepsilon^{-1}\left(\gamma-\gamma_{0}\right) \\
& y=\eta_{0}+\mu^{-1} \gamma_{0} \beta_{0} \sin \psi_{0} \sin \left(\phi_{0}+\mu \lambda\right) \\
& z=\zeta_{0}-\mu^{-1} \gamma_{0} \beta_{0} \sin \psi_{0} \cos \left(\phi_{0}+\mu \lambda\right)
\end{aligned}
$$

This is a spiral on the same cylinder as in the absence of $E$ (Part II), but of non-uniform pitch, the $x$-displacement being exactly that in the absence of H (Part I). 
Guide to Computation I I (A) $\left(E_{1}, \dot{*}, 0\right),(-H, 0,0)$

Given $R_{0}, \Psi_{0}, k_{0}^{\prime}, e^{\prime}, E_{1}^{\prime}, \varepsilon= \pm n E_{1}^{\prime} / e^{\prime}, \quad \mu= \pm 3 n \times 10^{-4}\left(H / e^{\prime}\right), \tau$

a. $\quad \gamma_{0}=1+\left(k_{0}^{\prime} / e^{\prime}\right), \quad \beta_{0}=\left(1-\gamma_{0}^{-2}\right)^{\frac{1}{2}}$

b. $\quad A=\gamma_{0} / \varepsilon, T=\tau / A, \quad \omega=\mu / \gamma_{0}$

c. $\quad \mathrm{G}=\left\{1+T\left(2 B_{0} a^{o}+T\right)\right\}^{\frac{1}{2}} \quad\left(=\frac{\gamma}{\gamma_{0}}\right)$

d. $\quad J=\ln \left\{\left(T+\beta_{0} a_{x}^{o}+G\right) /\left(1+\beta_{0} a_{x}^{o}\right)\right\}$

e. $\quad C=\cos \mu \varepsilon^{-1} L, \quad S=\sin \mu \varepsilon^{-1} L$

f. $\quad B=\left(B_{0}^{-1} T+a_{x}^{o}\right)^{2}+\left(a_{y}^{0}\right)^{2}+\left(a_{z}^{o}\right)^{2}$

g. $\left(a_{x}, a_{y}, a_{z}\right)=\left(B_{0}^{-1} T+a_{x}^{o}, a_{y}^{o} C-a_{z}^{o} S, a_{z}^{o} C+a_{y}^{o} S\right) / B$

h. $x=x_{0}+A(G-1), \quad k^{\prime}=k_{0}^{\prime} \pm n E_{1}^{\prime}\left(x-x_{0}\right)$

i. $y=y_{0}+w^{-1} \beta_{0}\left(a_{y}^{o} S-a_{z}^{o}(1-C)\right)$

$==z_{0}+\omega^{-1} B_{0}\left(a_{z}^{o} S+a_{y}^{o}(1-C)\right)$

Note 6. The non-relativistic version of the motion in the parallel case IIIA results from the force law

$$
\mathrm{d}\left(\mathrm{mv}_{\mathrm{x}}, \mathrm{mv_{y }}, \mathrm{mv}_{z}\right) / \mathrm{dt}=\left(\mathrm{QE}_{1},-Q H v_{z} / \mathrm{c}, Q H v_{y} / \mathrm{c}\right)
$$

or, in our notation 


$$
d\left(\beta_{x}, \beta_{y}, \beta_{z}\right) / d \tau=\left(E,-\mu \beta_{z}, \mu \beta_{y}\right)
$$

This implies the trajectory

$$
\begin{aligned}
& x=x_{0}+\beta_{0} a_{x}^{0} \tau+\frac{1}{2} \varepsilon \tau^{2} \\
& y=\eta_{0}+\mu^{-1} \beta_{0}\left(a_{z}^{0} \cos \mu \tau+a_{y}^{0} \sin \mu \tau\right), \eta_{0}=y_{0}-\mu^{-1} \beta_{0} a_{z}^{0} \\
& z=\zeta_{0}-\mu^{-1} \beta_{o}\left(a_{y}^{0} \cos \mu \tau-a_{z}^{0} \sin \mu \tau\right), \zeta_{0}=z_{0}+\mu^{-1} \beta_{0} a_{y}^{0}
\end{aligned}
$$

For the initial conditions $R_{0}=(0,0,0), \Psi_{0}=(0,1,0)$ perpendicular to the fields, and $\beta=\beta_{0}>0$, we see that

$$
\begin{aligned}
& x=\frac{1}{2} \varepsilon \tau^{2} \\
& y=\mu^{-1} \beta_{0} \sin \mu \tau \\
& z=\mu^{-1} \beta_{0}(1-\cos \mu \tau)
\end{aligned}
$$

This is a spiral of non-uniform pitch, on a circular cylinder of radius $\mu^{-1} \beta_{0}$, and axis parallel to the fields through the point $\left(0,0, \mu^{-1} \beta_{0}\right)$. If for $\mu \tau \ll 1$ we use the approximations

$$
x=\frac{1}{2} \varepsilon \tau^{2} \quad y=\mu^{-1} \beta_{0}(\mu \tau)=\beta_{0} \tau \quad z=\mu^{-1} \beta_{0}\left(\frac{1}{2} \mu^{2} \tau^{2}\right)=\frac{1}{2} \beta_{0} \mu \tau^{2}
$$

we see that the trajectory cuts the plane $y=L$ at $\tau=\beta_{0}^{-1} L$, in a point with

$$
x=\frac{1}{2} \epsilon \beta_{0}^{-2} L^{2} \quad z=\frac{1}{2} \mu \beta_{0}^{-1} L^{2}
$$


Elimination of $B_{0}$ gives

$$
z^{2}=\left(\frac{1}{2} E^{-1} \mu^{2} L^{2}\right) x=\left(\frac{1}{2} E_{1}^{-1} H^{2} L^{2}\right)(Q / e) x
$$

This is the parabolic locus of the intersections of the trajectories with the plane $y=L$ for particles with the same charge $Q$ but different initial velocities $\left(0, B_{0} c, 0\right)$. Otjervation of the parabolic trace permits evaluation of the charge to mass ratio $Q / m$ of the particles, a method used by J. J. Thomson. 
III B. Perpendicular Case. Assuming $H=(0, H, 0), H>0$, the force law (50) may be written as

$$
d(X, Y, Z) / d \lambda=(\varepsilon Y-\mu Z, 0, \mu X)
$$

Trivially therefore

$$
Y=Y_{0} \quad y=Y_{0}+Y_{0} \lambda
$$

Now the relation $y-Y_{0}=\varepsilon\left(x-x_{0}\right)$ implies that

$$
d \gamma / d \lambda=\varepsilon d x / d \lambda=\varepsilon X
$$

Hence, differentiating the equations

$$
\mathrm{dX} / \mathrm{d} \lambda=\varepsilon \gamma-\mu \mathrm{d} \quad \mathrm{d} Z / \mathrm{d} \lambda=\mu \mathrm{X}
$$

results in the system

$$
d^{2} x / d \lambda^{2}+\left(\mu^{2}-\varepsilon^{2}\right) x=0 \quad d^{2} z / d \lambda^{2}+\mu^{2} z=\mu \varepsilon \gamma
$$

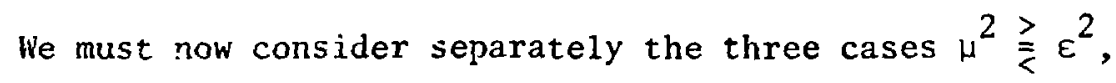
(i.e., $H \gtreqless E_{1}$ ) which result in quite different motions. In all three, we shall employ the folloning uniform strategy:

a. Solution of (71a) for $X(\lambda)$, determining its two constants from $x(0)=x_{0}$, and substitution in (70a) with $\lambda=0$. 
b. Determination of $x(\lambda)$ by integration of

$$
\mathrm{dx} / \mathrm{d} \lambda=\mathrm{x}(\lambda)
$$

c. Obtaining $\gamma(\lambda)$ and $k(\lambda)$ from

$$
\gamma-\gamma_{0}=\varepsilon\left(x-x_{0}\right), \quad k-k_{0}=Q E_{1}\left(x-x_{0}\right)
$$

d. Finding $Z(\lambda)$ by substitution of $d x / d \lambda$ and $\gamma(\lambda)$ in (70a)

e. Determination of $z(\lambda)$ by integration of

$$
d z / d \lambda=z(\lambda)
$$

In this way, no use is made of (71b).

These steps may be carried through in explicit form to give all variables as functions of $\lambda$. Unfortunately the dependence of $\lambda$ on $\tau$ is of an implicit nature. Since $d \tau / d \lambda=\gamma$, we have

$$
\tau=\int_{0}^{\lambda} \gamma(\lambda) \mathrm{d} \lambda \equiv \Gamma(\lambda)
$$

in terms of the known $\gamma(\lambda)$. Thus we require also the step

$$
\text { f. } \lambda=\Gamma^{-1}(\tau)
$$

a relation requiring approximation methods in the cases $H_{<}^{>} E_{1}$. Such methods are not discussed below. 


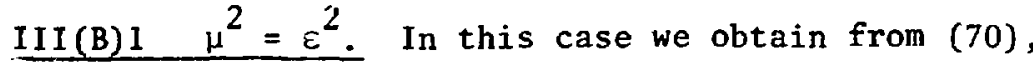

$$
\begin{aligned}
& d X / d \lambda=\mu(\gamma-Z) \\
& d^{2} X / d \lambda^{2}=0
\end{aligned}
$$

Following the strategy indicated, we find

$$
\begin{aligned}
& X(\lambda)=x_{0}+x_{0}^{\prime} \lambda \quad x_{0}^{\prime} \equiv \mu\left(Y_{0}-z_{0}\right) \\
& x(\lambda)=x_{0}+X_{0} \lambda+\frac{1}{2} X_{0}^{\prime} \lambda^{2} \\
& \gamma(\lambda)=\gamma_{0}+\mu X_{0} \lambda+\frac{1}{2} \mu X_{0}^{\prime} \lambda^{2} \\
& Z(\lambda)=z_{0}+\mu X_{0} \lambda+\frac{1}{2} \mu X_{0}^{\prime} \lambda^{2} \\
& z(\lambda)=z_{0}+z_{0} \lambda+\frac{1}{2} \mu X_{0} \lambda^{2}+\frac{1}{6} \mu X_{0}^{\prime} \lambda^{3} \\
& Y=Y_{0} y=y_{0}+Y_{0} \lambda \\
& \tau(\lambda)=Y_{0} \lambda+\frac{1}{2} \mu X_{0} \lambda^{2}+\frac{1}{6} \mu X_{0}^{\prime} \lambda^{3}
\end{aligned}
$$

Guide to Computation. III (B) $1 \quad\left(E_{1}, 0,0\right),(0, H, 0) \quad H=E_{1}$

Given $R_{0}, \Psi_{0}, k_{0}^{\prime}, e^{\prime}, E_{1}^{\prime}, \mu= \pm 3 n \times 10^{-4}\left(H / e^{\prime}\right), \tau$

a. $\gamma_{0}=1+\left(k_{0}^{\prime} / e^{\prime}\right) \quad \beta_{0}=\left(1-\gamma_{0}^{-2}\right)^{\frac{1}{2}}$

b. $\quad\left(\beta_{x}^{0}, \beta_{y}^{0}, \beta_{z}^{0}\right)=\beta_{0}\left(a_{x}^{0}, a_{y}^{0}, a_{z}^{0}\right)$

c. $\tau=\gamma_{0} \lambda \div \frac{1}{2} \mu \gamma_{0} \beta_{x}^{0} \lambda^{2}+\frac{1}{6} \mu^{2} \gamma_{0}\left(1-\beta_{z}^{0}\right) \lambda^{3} \equiv \Gamma(\lambda)$

$$
\lambda=\Gamma^{-1}(\tau)
$$


d. $B_{x}=\beta_{x}^{O}+\mu \lambda\left(1-B_{z}^{O}\right)$

$$
\begin{aligned}
& \left.B_{z}=\beta_{z}^{O}+i\right)\left\{\beta_{x}^{O}+\frac{1}{2}\left(\lambda\left(1-Q_{z}^{O}\right)\right\}\right. \\
& B=B_{x}^{2}+\left(\beta_{y}^{O}\right)^{2}+B_{z}^{2}
\end{aligned}
$$

e. $\quad\left(a_{x}, a_{y}, a_{z}\right)=\left(B_{x}, B_{y}^{O}, B_{z}\right) / B$

f. $\Delta x=\gamma_{o} \lambda\left\{\beta_{x}^{0}+\frac{1}{2} \mu \lambda\left(1-B_{z}^{O}\right)\right\}$

$$
x=x_{0}+\Delta x \quad k^{\prime}=k_{0}^{\prime} \pm n E_{j}^{\prime} \Delta x
$$

g. $\quad y=y_{0}+\gamma_{0} \beta_{y}^{o} \lambda$

h. $z=z_{0}+\tau-Y_{0}\left(1-\beta_{z}^{0}\right) \lambda$

Note 7. The routine for the perpendicular cases $I I I(B) 1,2,3$ require solution of an equation

$$
\tau=\int_{0}^{\lambda} \gamma(\lambda) \mathrm{d} \lambda \equiv \Gamma(\lambda)
$$

for $\lambda>0$ in terms of a given $\tau>0$, the function $\Gamma(\lambda)$ being strictly increasing with

$$
\Gamma^{\prime}(\lambda)=\gamma(\lambda) \geq 1, \quad \Gamma(0)=0
$$

If desired, this can be done algebraically in the present case $(\mu=\varepsilon)$, since from (82) 


$$
\tau=\gamma_{0} \lambda+\frac{1}{2} \mu X_{0} \lambda^{2}+\frac{1}{6} \mu X_{0}^{\prime} \lambda^{3} ; \quad X_{0}^{\prime}=\mu\left(\gamma_{0}-Z_{0}\right)
$$

which may be written as the cubic equation

$$
\xi^{3}+\mathrm{b} \xi^{2}+\mathrm{c} \xi+\mathrm{d}:=0, \quad \xi=\mu \lambda
$$

where

$$
\begin{array}{ll}
b=3 \alpha / \beta & c=6 / \beta \quad d=-0 \mu \tau / \gamma_{0} \beta \\
\alpha \equiv \beta_{x}^{0} & \beta \equiv 1-\beta_{z}^{0}>0
\end{array}
$$

For $\xi=\eta-b / 3=\eta-\alpha / \beta$ we obtain the reduced cubic

$$
n^{3}+p n+q=0
$$

where $p=c-b^{2} / 3=3\left(2 \beta-\alpha^{2}\right) / \beta^{2}$

$$
q=d-\frac{b}{9}(c+2 p)=-\frac{6 \mu \tau}{\gamma_{0} \beta}-\frac{2 \alpha}{\beta^{3}}\left(3 \beta-\alpha^{2}\right)
$$

Note that $\beta=1 \cdots \beta_{z}^{0}>0$ and $p=3\left(2 \beta-\alpha^{2}\right) / \beta^{2}=\frac{3}{\beta^{2}}\left(2-2 \beta_{z}^{0}-\left(\beta_{x}^{0}\right)^{2}\right) .>0$. In fact,

$$
\begin{aligned}
0 & \leq\left(\beta_{x}^{0}\right)^{2} \leq \beta_{0}^{2}-\left(\beta_{z}^{0}\right)^{2}=-\left(1-\beta_{0}^{2}\right)+\left(1-\left(\beta_{z}^{0}\right)^{2}\right) \\
& <1-\left(\beta_{z}^{O}\right)^{2}<2\left(1-\beta_{z}^{0}\right) .
\end{aligned}
$$

It follows that $w=(p / 3)^{3}+(q / 2)^{2}>0$, and such a cubic has just one real root, namely 
$-46-$

$$
\begin{aligned}
& \eta=\hat{H}+\hat{J} \\
& \hat{H}=\left(-\frac{q}{2}+w^{1 / 2}\right)^{1 / 3}, \quad \hat{J}=-p / 3 H
\end{aligned}
$$

Hence from $\beta_{x}^{0}, 1-\beta_{z}^{o}, \mu, \gamma_{0}$, and a given $\tau$, one may compute $\alpha, \beta, p, q, w$, $\hat{H}, \hat{J}, \eta, \xi=\eta-\alpha / \beta$, and

$$
\lambda=\xi / \mu
$$


III(B) 2. $\mu^{2}>\varepsilon^{2}$. Equations (70), (71) now yield

$$
\begin{aligned}
& d x / d \lambda=\varepsilon \gamma-\mu Z \\
& d^{2} x / d \lambda^{2}+\delta^{2} x=0, \quad \delta^{2} \equiv \mu^{2}-\varepsilon^{2}>0
\end{aligned}
$$

Our method shows that

$$
\begin{aligned}
& x(\lambda)=x_{0} \cos \delta \lambda+x_{0}^{*} \sin \delta \lambda, \quad x_{0}^{*} \equiv \delta^{-1}\left(\varepsilon y_{0}-\mu z_{0}\right) \\
& x(\lambda)=\xi_{0}+\delta^{-1}\left(x_{0} \sin \delta \lambda-x_{0}^{*} \cos \delta \lambda\right), \quad \xi_{0}=x_{0}+\delta^{-1} x_{0}^{*} \\
& \gamma(\lambda)=G_{0}+\varepsilon \delta^{-1}\left(x_{0} \sin \delta \lambda-x_{0}^{*} \cos \delta \lambda\right) \\
& G_{0}=\gamma_{0}+\varepsilon \delta^{-1} x_{0}^{*}=\mu \delta^{-2}\left(\mu \gamma_{0}-\varepsilon Z_{0}\right)>0 \\
& Z(\lambda)=\varepsilon G_{0} / \mu+\mu \delta^{-1}\left(x_{0} \sin \delta \lambda-X_{0}^{*} \cos \delta \lambda\right) \\
& z(\lambda)=\zeta_{0}+\varepsilon \mu^{-1} G_{0} \lambda-\mu \delta^{-2}\left(X_{0} \cos \delta \lambda+X_{0}^{*} \sin \delta \lambda\right) \\
& \zeta_{0}=z_{0}+\mu \delta^{-2} x_{0} \\
& \tau(\lambda)=\varepsilon \delta^{-2} X_{0}+G_{o} \lambda-\varepsilon \delta^{-2}\left(X_{0} \cos \delta \lambda+X_{o}^{*} \sin \delta \lambda\right) \equiv \Gamma(\lambda) \\
& Y=Y_{0} \quad y=y_{0}+Y_{0} \lambda
\end{aligned}
$$

Assuming $x_{0}^{2}+x_{0}^{* 2}>0\left(\beta_{x}^{\circ} \neq 0, \varepsilon \neq \mu B_{z}^{0}\right)$ we may define

$$
A_{0}=\left(x_{0}^{2}+x_{0}^{*}\right)^{\frac{1}{2}}, \cos \theta_{0}=x_{0} / A_{0}, \sin \theta_{0}=x_{0}^{*} / A_{0}
$$




$$
\begin{aligned}
& x=\xi_{0}+\delta^{-1} A_{0} \sin \left(\hat{o} \lambda-\theta_{0}\right) \\
& y=y_{0}+\gamma_{0} \lambda \\
& z=\zeta_{0}+\varepsilon \mu^{-1} \sigma_{0} \lambda-\mu \delta^{-2} A_{0} \cos \left(\delta \lambda-\theta_{0}\right)
\end{aligned}
$$

This may be visualized as an elliptical spiral with axis in the direction of $H$, undergoing a drift in the $z$-direction.

In the very special case $x_{0}^{2}+x_{0}^{* 2}=0$, the trajectory is the straight ] ine

$$
x=x_{0}, \quad y=y_{0}+Y_{0} \lambda, \quad z=z_{0}+\varepsilon \mu^{-1} \gamma_{0} \lambda
$$

in the plane $x=x_{0}$.

Guide to Computation. III (B) 2. $\left(E_{1}, 0,0\right),(0, \mathrm{H}, 0), \mathrm{H}>\mathrm{s}_{1}$

Given $R_{0}, \psi_{0}, k_{o}^{\prime}, e^{\prime}, E_{1}^{\prime}, \delta=\left(\mu^{2}-\varepsilon^{2}\right)^{\frac{1}{2}}, \varepsilon_{1}=\varepsilon / \delta, \mu_{1}=\mu / \delta, \mu_{11}=\mu / \delta^{2}, \tau$

a. $\gamma_{0}=1+\left(k_{0}^{\prime} / e^{\prime}\right) \quad \beta_{0}=\left(1-\gamma_{0}^{-2}\right)^{\frac{1}{2}}$

b. $\quad\left(\beta_{x}^{0}, \beta_{y}^{0}, \beta_{z}^{0}\right)=\beta_{0}\left(a_{x}^{o}, a_{y}^{o}, a_{z}^{o}\right)$

c. $\quad X_{1}^{*}=\varepsilon_{1}-\mu_{1} \beta_{z}^{o} \quad G_{1}=\varepsilon_{1}\left(\mu_{1}-\varepsilon_{1} \beta_{2}^{o}\right)$

d. $\tau=\gamma_{0}\left\{\left(\mu_{1} / \varepsilon_{1}\right) G_{1} \lambda+\left(E_{1} / \delta\right)\left[\beta_{x}^{o}(1-\cos \delta \lambda)-x_{1}^{*} \sin \delta \lambda\right]\right\} \equiv \Gamma(\lambda)$

$$
\lambda=\Gamma^{-1}(\tau)
$$

e. $\quad \mathrm{C}=\cos \delta \lambda \quad S=\sin \delta \lambda$ 
f. $B_{x}=B_{x}^{o} C+X_{1}^{*} S \quad B_{z}=G_{1}+\mu_{1}\left(B_{x}^{o} S-X_{1}^{*} C\right)$

$$
B=\left\{B_{x}^{2}+\left(B_{y}^{0}\right)^{2}+B_{z}^{2}\right\}^{\frac{1}{2}}
$$

g. $\quad\left(a_{x}, a_{y}, a_{z}\right)=\left(B_{x}, B_{y}^{O}, B_{z}\right) / B$

h. $\Delta x=\delta^{-1} \gamma_{0}\left\{B_{X}^{o} S+X_{1}^{*}(1-C)\right\}$

$$
\mathrm{x}=\mathrm{x}_{\mathrm{o}}+\Delta \mathrm{x} \quad \mathrm{k}^{\prime}=\mathrm{k}_{0}^{\prime} \pm \mathrm{nE}_{1}^{\prime} \Delta \mathrm{x}
$$

i. $\quad y=y_{0}+\gamma_{0} \beta_{y}^{o} \lambda$

j. $\quad z=z_{0}+Y_{0}\left\{G_{1} \lambda+\mu_{11}\left[\beta_{x}^{o}(1-C)-X_{1}^{*} S\right]\right\}$

Note 8. While we include no approximation methods for inverting the relation $\because=\Gamma(\lambda)$, it may be noted that (90) may be written in the form

$$
\begin{gathered}
\delta \tau / \gamma_{0}=\varepsilon_{1} \beta_{x}^{0}+\mu_{1} C_{11} \xi-\varepsilon_{1}\left(\beta_{x}^{0} \cos \xi+B_{11} \sin \xi\right) \equiv F(\xi) ; \quad \xi=\delta \lambda \\
\varepsilon_{1}=\varepsilon / \delta \quad \mu_{1}=\mu / \delta \quad \mu_{1}^{2}-\varepsilon_{1}^{2}=1 \\
C_{11}=\mu_{1}-\varepsilon_{1} \beta_{z}^{0}>0 \quad B_{11}=\varepsilon_{1}-\mu_{1} \beta_{z}^{0}
\end{gathered}
$$

The function $F(\xi)$ is strictly increasing, with

$$
F(0)=0 \quad F^{\prime}(\xi)=\gamma / \gamma_{0} \geq 1 / \gamma_{0}>0 \quad F^{\prime}(0)=1 \quad F^{\prime \prime}(0)=\varepsilon_{1} \beta_{x}^{0}
$$


III (B) $3 \quad \mu^{2}<\varepsilon^{2}$. We now have from (70), (71)

$$
\begin{aligned}
& \mathrm{dX} / \mathrm{d} \lambda=\varepsilon \gamma-\mu \mathrm{Z} \\
& \mathrm{d}^{2} \mathrm{x} / \mathrm{d} \lambda^{2}-\delta^{2} \mathrm{x}=0 \quad \delta^{2}=\varepsilon^{2}-\mu^{2}>0
\end{aligned}
$$

and obtain by the standard method

$$
\begin{aligned}
& x(\lambda)=A e^{\delta \lambda}+B e^{-\delta \lambda} \quad A=\frac{1}{2}\left(X_{0}+X_{0}^{*}\right), \quad B=\frac{1}{2}\left(X_{0}-X_{0}^{*}\right) \\
& X_{0}^{*} \equiv \delta^{-1}\left(\varepsilon \gamma_{0}-\mu Z_{0}\right)>0 \\
& x(\lambda)=\xi_{0}+\delta^{-1}\left(A e^{\delta \lambda}-B e^{-\delta \lambda}\right), \quad \xi_{0}=x_{0}-\delta^{-1} x_{0}^{*} \\
& \gamma(\lambda)=G_{0}+\varepsilon \delta^{-1}\left(A e^{\delta \lambda}-B e^{-\delta \lambda}\right) \quad G_{0}=\gamma_{0}-\varepsilon \delta^{-1} x_{0}^{*}=-\mu \delta^{-2}\left(\mu \gamma_{0}-\varepsilon Z_{0}\right) \\
& Z(\lambda)=\varepsilon G_{0} / \mu+\mu \delta^{-1}\left(A e^{\delta \lambda}-B e^{-\delta \lambda}\right) \\
& z(\lambda)=\zeta_{0}+\varepsilon \mu^{-1} G_{0} \lambda+\mu \delta^{-2}\left(A e^{\delta \lambda}+B e^{-\delta \lambda}\right), \zeta_{0}=z_{0}-\mu \delta^{-2} X_{0} \\
& Y=Y_{0} \quad y=y_{0}+Y_{0} \lambda \\
& \tau(\lambda)=-\varepsilon \delta^{-2} x_{0}+G_{0} \lambda+\varepsilon \delta^{-2}\left(A e^{\delta \lambda}+B e^{-\delta \lambda}\right) \equiv \Gamma(\lambda)
\end{aligned}
$$


$-51-$

Guide to Computation. III (B) $3 \quad\left(E_{1}, 0,0\right),(0, H, 0), H<E_{1}$

Given $R_{0}, \Psi_{0}, k_{0}^{\prime}, e^{\prime}, E_{1}^{\prime}, \delta=\left(\varepsilon^{2}-\mu^{2}\right)^{\frac{1}{2}}, \varepsilon_{1}=\varepsilon / \delta, \mu_{1}=\mu / \delta, \mu_{11}=\mu / \delta^{2}, \tau$

a. $\gamma_{0}=1+\left(k_{0}^{\prime} / e^{\prime}\right) \quad \beta_{0}=\left(1-\gamma_{0}^{-2}\right)^{\frac{1}{2}}$

b. $\quad\left(\beta_{x}^{0}, \beta_{y}^{0}, \beta_{z}^{0}\right)=\beta_{j}\left(a_{x}^{0}, a_{y}^{0}, a_{z}^{0}\right)$

c.

$$
\begin{array}{ll}
x_{1}^{*}=\varepsilon_{1}-\mu_{1} \beta_{z}^{0}, & G_{1}=-\varepsilon_{1}\left(\mu_{1}-\varepsilon_{1} \beta_{z}^{\circ}\right) \\
A_{1}=\frac{1}{2}\left(\beta_{x}^{0}+X_{1}^{*}\right), & B_{1}=\frac{1}{2}\left(\beta_{x}^{0}-x_{1}^{*}\right)
\end{array}
$$

d. $\quad r=\gamma_{0}\left\{\left(\mu_{1} / \epsilon_{1}\right) G_{1} \lambda+\left(\varepsilon_{1} / \delta\right)\left[-\beta_{x}^{o}+A_{1} e^{\delta \lambda}+B_{1} e^{-\delta \lambda}\right]\right\} \equiv \Gamma(\lambda)$

$$
\lambda=\Gamma^{-1}(\tau)
$$

e. $\quad c=e^{\delta \lambda}, \quad s=i / z$

f. $\quad B_{x}=A_{1} C+B_{1} S, B_{z}=G_{1}+\mu_{1}\left(A_{1} C-B_{1} S\right)$

$$
B=\left\{B_{x}^{2}+\left(\beta_{y}^{0}\right)^{2}+B_{z}^{2}\right\}^{\frac{1}{2}}
$$

g. $\left(a_{x}, a_{y}, a_{z}\right)=\left(B_{x}, B_{y}^{O}, B_{z}\right) / B$

h. $\Delta x=\gamma_{0} \delta^{-1}\left(-x_{1}^{*}+A_{1} C-B_{1} S\right)$

$$
x=x_{0}+\Delta x \quad k^{\prime}=k_{0}^{\prime} \pm n_{1}^{\prime} \Delta x
$$

i. $y=y_{0}+\gamma_{0} \beta_{y}^{o} \lambda$

j. $\quad z=z_{c}+\gamma_{0}\left\{G_{1} \lambda+\mu_{11}\left(A_{1} C+B_{1} S-B_{x}^{0}\right)\right\}$ 
Note 9. In the inversion of $\tau=\Gamma(\lambda)$, we note that (102) may be written in the form

$$
\begin{gathered}
\delta \tau / \gamma_{0}=-\varepsilon_{1} \beta_{x}^{o}+\mu_{1} C_{11} \xi+\varepsilon_{1}\left(A_{11} e^{\xi_{3}+B_{11}} e^{-\xi}\right) \equiv F(\xi) ; \quad \xi=\delta \gamma \\
\varepsilon_{1}=\varepsilon / \delta \quad \mu_{1}=11 / \delta \quad \varepsilon_{1}^{2}-\mu_{1}^{2}=1 \\
C_{11}=\varepsilon_{1} \beta_{z}^{o}-\mu_{1} \\
A_{11}=\left(\beta_{x}^{o}+D_{11}\right) / 2 \quad B_{11}=\left(\beta_{x}^{o}-D_{11}\right) / 2 \quad D_{11}=\varepsilon_{1}-k_{1} \beta_{z}^{o}>0
\end{gathered}
$$

The function $F(\xi)$ is strictly increasing, with

$$
F(0)=0 \quad F^{\prime}(\xi)=\gamma / \gamma_{0} \geq 1 / \gamma_{0}>0 \quad F^{\prime}(0)=1 \quad F^{\prime \prime}(0)=\varepsilon_{1} \beta_{x}^{0} .
$$

It can be shown* that $B_{11}<0<A_{11}$, and therefore

$$
F^{\prime \prime}(\xi) \equiv \varepsilon_{1}\left(A_{11} e^{\xi}+B_{11} e^{-\xi}\right)=0 \text { for } e^{2 \xi}=-B_{11} / A_{11}>0
$$

Moreover $-B_{11} / A_{11}>1$ iff $A_{11}+B_{11}=B_{x}^{O}<0$. Thus $F(x)$ is concave up for al1 $\xi>0$ if $\beta_{x}^{0} \geq 0$, and has a ringle inflection point at $\xi=(1 / 2) \log \left(-B_{11} / A_{11}\right)$ if $\beta_{x}^{\circ}<0$.

$*_{0}<\gamma_{0}^{-2}+C_{11}^{2} \equiv D_{11}^{2}-\left(\beta_{0}^{2}-\left(\beta_{z}^{0}\right)^{2}\right) \leq D_{11}^{2}-\left(\beta_{x}^{0}\right)^{2}$ 
III(C). Arbitrary Orientation. Having considered in III(A), (B) the parallel and perpendicular cases, it is clear that all other orientations of the superimposed fields are included under the equation

$$
\dot{\mathrm{P}}=\mathrm{F}=\mathrm{QE}+\mathrm{Q} \overline{\mathrm{V}} \times \mathrm{H}
$$

where $E=\left(E_{1}, 0,0\right), E_{1}>0$, and

$$
\begin{array}{ll}
H=(H C, H S, 0), & H>0 \\
C=\cos \theta, & S=\sin \theta \\
0<\theta<\pi, & \theta \neq \pi / 2
\end{array}
$$

We recall that the relations

$$
k-k_{0}=Q_{1}\left(x-x_{0}\right), \quad \gamma-\gamma_{0}=\varepsilon\left(x-x_{0}\right), \quad d \gamma / d \lambda=\varepsilon X
$$

are still valid. For the stipulated fields, the force law in our notation becomes

$$
\left(X^{\prime}, Y^{\prime}, Z^{\prime}\right)=(E Y-\mu S Z, \mu C Z, \mu(S X-C Y))
$$

where primes hereafter indicate derivatives with respect to $\lambda$. Differentiating in (103), we see that

$$
\begin{array}{ll}
X^{\prime \prime}=A_{1} X+B_{1} Y & A_{1}=\varepsilon^{2}-\mu^{2} S^{2} \quad B_{1}=\mu^{2} S C \neq 0 \\
Y^{\prime \prime}=B_{1} X+C_{1} Y & C_{1}=-\mu^{2} C^{2}<0
\end{array}
$$


the determinant of the system being

$$
\Delta=A_{1} C_{1}-B_{1}^{2}=-\mu^{2} \varepsilon^{2} C^{2}<0
$$

and moreover

$$
Z^{\prime \prime}+\mu^{2} Z=\mu E S \gamma(\lambda)
$$

The form of (104) suggests solutions $X, Y$ which are linear combinations of trigonometric and exponential functions. If we assume

$$
\begin{array}{rlr}
X=u & +V \\
U & =U_{1} \cos K \lambda+U_{2} \sin K \lambda & K>0 \\
V & =V_{1} e^{L \lambda}+V_{2} e^{-L \lambda} & L>0
\end{array}
$$

with $Y=\hat{U}+\hat{V}$ a similar combination, substitution in (104) requires

$$
\begin{aligned}
& -K^{2} U+L^{2} V=A_{1}(U+V)+B_{1}(\hat{U}+\hat{V}) \\
& -K^{2} \hat{U}+L^{2} \hat{V}=B_{1}(U+V)+C_{1}(\hat{U}+\hat{V})
\end{aligned}
$$

This will be true provided

$$
Y=\hat{u}+\hat{V}=c U+d V
$$

where

$$
c=-\left(K^{2}+A_{1}\right) / B_{1} \quad d=\left(L^{2}-A_{1}\right) / B_{1}
$$


and $\left(K^{2}+A_{1}\right) / B_{1}=B_{1} /\left(K^{2}+C_{1}\right), \quad\left(L^{2}-A_{1}\right) / B_{1}=B_{1} /\left(L^{2}-C_{1}\right)$.

The latter require that (cf (104))

$$
\begin{gathered}
\mathrm{K}^{2}=\frac{1}{2}\left\{-\left(\varepsilon^{2}-\mu^{2}\right)+\mathrm{R}\right\}, \quad \mathrm{L}^{2}=\frac{1}{2}\left\{\left(\varepsilon^{2}-\mu^{2}\right)+\mathrm{R}\right\} \\
\mathrm{R} \equiv\left\{\left(\varepsilon^{2}-\mu^{2}\right)^{2}+4 \mu^{2} \varepsilon^{2} C^{2}\right\}^{\frac{1}{2}}
\end{gathered}
$$

Hence for the positive values of $\mathrm{K}, \mathrm{L}$ in (110), and the resulting values of $c, d$ in (109), we obtain a solution $X, Y$ from (107) and (108) involving four arbitrary constants

$$
U_{i}, v_{i} ; \quad i=1,2
$$

The following relations are readily verified from the definitions. Some are useful in evaluating later constants.

$$
\begin{array}{ll}
K^{2}+A_{1}>0 & L^{2}-A_{1}>0 \\
\varepsilon^{2}+\mu^{2}>R \quad & \mu^{2}>K^{2} \\
K^{2}-L^{2}=\mu^{2}-\varepsilon^{2} \quad K^{2} L^{2}=\mu^{2} \varepsilon^{2} C^{2} \\
d-c=\left(K^{2}+L^{2}\right) / B_{1}=R / B_{1} \quad c d=-1 \\
c K^{2}+d L^{2}=-\mu^{2} C^{2}\left(K^{2}+L^{2}\right) / B_{1} \\
d K^{2}+c L^{2}=-A_{1}\left(K^{2}+L^{2}\right) / B_{1}
\end{array}
$$

It remains to determine the four constants $u_{i}, v_{i}$. Using (107), (108), and (103), we obtain the linear equations 


$$
\begin{aligned}
& \mathrm{u}_{1}+\mathrm{v}_{1}+\mathrm{v}_{2}=\mathrm{x}_{\mathrm{o}} \\
& c U_{1}+d V_{1}+d v_{2}=Y_{0} \\
& \mathrm{KU}_{2}+L V_{1}-L V_{2}=X_{0}^{\prime}=\varepsilon \gamma_{0}-\mu S Z_{0} \\
& \mathrm{cKU}_{2}+\mathrm{dLV}_{1}-\mathrm{dLV}_{2}=\mathrm{Y}_{\mathrm{o}}^{\prime}=\mu \mathrm{CZ} \mathrm{o}_{\mathrm{o}}
\end{aligned}
$$

with determinant

$$
\Delta^{\prime}=2 K L(d-c)^{2}=2 K L\left(K^{2}+L^{2}\right)^{2} / B_{1}^{2}>0
$$

Solving the above system gives the explicit constants

$$
\begin{aligned}
& U_{1}=-\left(Y_{0}-d X_{0}\right) /(d-c) \\
& U_{2}=-\left(Y_{0}^{\prime}-d X_{0}^{\prime}\right) / K(d-c) \\
& V_{1}=\left\{L\left(Y_{0}-c X_{0}\right)+\left(Y_{0}^{\prime}-c X_{0}^{\prime}\right)\right\} / 2 L(d-c) \\
& V_{2}=\left\{L\left(Y_{0}-c X_{0}\right)-\left(Y_{0}^{\prime}-c X_{0}^{\prime}\right)\right\} / 2 L(d-c)
\end{aligned}
$$

with $X_{0}^{\prime}, Y_{0}^{\prime}$ as in (111).

Integration of $\mathrm{d} x / \mathrm{d} \lambda=x(\lambda)$ now yields

$$
\begin{gathered}
x=\xi_{0}+K^{-1}\left(U_{1} \sin K \lambda-U_{2} \cos K \lambda\right)+L^{-1}\left(V_{1} e^{L \lambda}-V_{2} e^{-L \lambda}\right) \\
\xi_{0}=x_{0}+x_{1}, \quad x_{1} \equiv K^{-1} U_{2}-L^{-1}\left(V_{1}-V_{2}\right)=-\gamma_{0} / \varepsilon
\end{gathered}
$$

From this and the relation $\gamma-\gamma_{0}=\varepsilon\left(x-x_{0}\right)$, we obtain 


$$
\gamma=\varepsilon K^{-1}\left(U_{1} \sin K \lambda-U_{2} \cos K \lambda\right)+\varepsilon L^{-1}\left(V_{1} e^{L \lambda}-V_{2} e^{-L \lambda}\right)
$$

and therefore from $\tau=\int_{0}^{\lambda} \gamma(\lambda) d \lambda$

$$
\begin{aligned}
\tau=T_{1}-\varepsilon K^{-2} U+\varepsilon L^{-2} V \equiv \Gamma(\lambda) \\
T_{1}=\varepsilon\left\{K^{-2} U_{1}-L^{-2}\left(V_{1}+V_{2}\right)\right\}=-\left(C X_{0}+S Y_{0}\right) / \varepsilon C
\end{aligned}
$$

Frois: $d y / d \lambda=Y$ and (108) we find

$$
\begin{aligned}
& y=\eta_{0}+c K^{-1}\left(U_{1} \sin K \lambda-U_{2} \cos K \lambda\right)+d L^{-1}\left(V_{1} e^{L \lambda}-V_{2} e^{-L \lambda}\right) \\
& \eta_{0}=y_{0}+Y_{1}, \quad Y_{1}=c K^{-1} U_{2}-d L^{-1}\left(V_{1}-V_{2}\right)=\left(\varepsilon Z_{0}-\mu S \gamma_{0}\right) / \mu \varepsilon C
\end{aligned}
$$

Since $Y^{\prime}=\mu C Z$ in $(103)$, we may bypass $(106)$ and obtain $Z=(\mu C)^{-1} Y^{\prime}$. In this way, we find from (108),

$$
z=\frac{c K}{\mu C}\left(-U_{1} \sin K \lambda+U_{2} \cos K \lambda\right)+\frac{d L}{\mu C}\left(V_{1} e^{L \lambda}-V_{2} e^{-L \lambda}\right)
$$

and since $d z / d \lambda=Z(\lambda)$, we have

$$
\begin{aligned}
& z=\zeta_{0}+\frac{c}{\mu C} U+\frac{d}{\mu C} V \\
& \zeta_{0}=z_{0}-\left(\frac{c U_{1}}{\mu C}+\frac{d\left(V_{1}+V_{2}\right)}{\mu C}\right)=z_{0}-\frac{Y_{0}}{\mu C}
\end{aligned}
$$

It may be shown that

$$
c / \mu C=-\varepsilon^{2} \mu S / K^{2}\left(\mu^{2}-K^{2}\right), \quad d / \mu C=\varepsilon^{2} \mu S / L^{2}\left(\mu^{2}+L^{2}\right)
$$


(The bailistic trajectories of this section were obtained in a more complicated way in an earlier report LA-4967-MS. 
4. Motion against "friction." If a particle of pruper energy $e \geq 0$ starts from $R=0$ at time $t=0$ with initial momentum $P_{0}=p_{0} \Psi_{0}$, and is suhject thereafter to a "friction" $F=-H P, H>0$ constant, then the force law $\dot{\mathrm{P}}=\mathrm{F}=-\mathrm{HP}$ implies

$$
P=P_{0} \varepsilon^{-H t}
$$

Since $\mathrm{P}=\mathrm{p} \Psi$, we have for the magnitude and direction

$$
\mathrm{p}=\mathrm{p}_{0} \varepsilon^{-\mathrm{Ht}}, \quad \Psi \equiv \Psi_{0}
$$

Hence from $d R / d:=V=v \Psi=v_{0}^{\Psi}$ follows the 1inear trajectory

$$
R=s(t) \Psi_{0}, \quad s(t)=\int_{0}^{t} v(t) d t
$$

It remains to evaluate $v(t)$ and $s(t)$.

Case 1. e >0. For a material particle we know

$$
\begin{aligned}
& \left(\gamma^{2}-1\right)^{\frac{1}{2}}=\gamma \beta=c p / e=\frac{c p_{o}}{e} \cdot \varepsilon^{-H t}, \text { whence } \\
& v / c=\beta=\left(1-\gamma^{-2}\right)^{\frac{1}{2}}=\left\{1+\left(e / c p_{o}\right)^{2} \varepsilon^{2 H t}\right\}^{-\frac{1}{2}} \rightarrow 0
\end{aligned}
$$

Making the substitution

$$
\zeta=\zeta(\mathrm{t})=\left\{1+\left(\mathrm{e} / \mathrm{cp}_{\mathrm{o}}\right)^{2} \varepsilon^{2 \mathrm{Ht}}\right\}^{\frac{1}{2}}
$$




$$
s=\int_{0}^{t} v d t=\frac{c}{2 H} \ln \frac{(\zeta(0)+1)(\zeta(t)-1)}{(\zeta(0)-1)(\zeta(t)+1)}+\frac{c}{2 H} \ln \frac{\zeta(0)+1}{\zeta(0)-1}
$$

In computation based on initial k.e. $k_{0}$, one may note that $\gamma_{0}=1+\left(k_{0} / 2\right)$ and $\left(c_{0} / e\right)^{2}=\gamma_{0}^{2} B_{0}^{2}=\gamma_{0}^{2}-1$.

Case 2. $\quad \mathrm{e}=0$. As a purely mathematical exercise, which introduces some basic ideas of cosmology, we may consider the effect of such a friction on a photon of initial energy $E_{0}=c p_{0}=h v_{0}=h\left(c / \lambda_{0}\right)$. In this case we have trivially

$$
v \equiv c, \quad s=c t, \quad R=c t \Psi_{0}, \quad p=p_{0} \varepsilon^{-H t}
$$

and find an energy degradation expressed by the equation

$$
\begin{gathered}
E=E_{0} \varepsilon^{-H t}=E_{0} \varepsilon^{-\frac{H}{C} S}=E_{0} \varepsilon^{-S / S} \\
S \equiv \approx / H
\end{gathered}
$$

This would result in a longer wave length

$$
\lambda=\lambda_{0} \varepsilon^{s / S}>\lambda_{0}
$$

and consequently a "red shift"

$$
z \equiv \frac{\lambda-\lambda_{0}}{\lambda_{0}}=\varepsilon^{s / S}-1=(s / S)+(s / S)^{2} / 2 !+\ldots .
$$

Thus a friction $F=-H P$, acting on the $N_{0}$ photons, of average energy $E_{0}$, 
emitted per secund by a source of luminosity $L_{0}=\mathrm{N}_{0} \mathrm{E}_{0}$, at constant distance $s(M p c)$, would produce a flux

$$
\phi=N_{0} E_{0} \varepsilon^{-s / S} / 4 \pi s^{2}=L_{o} / 4 \pi s^{2} E^{s / S} \text { erg/sec }(M p c)^{2}
$$

exhibiting a red shift $z=\epsilon^{S / S}-1$. The distance indicated by an observed shift $z$ is therefore

$$
s=s \ln (1+z)
$$

Elimination of $s$ between (9) and (10) yields the flux-shift relation

$$
\phi=L_{0} / 4 \pi S^{2}(1+z) \ln ^{2}(1+z)
$$

Defining the apparent and absolute magnitudes $m, M$ of the source in the usual way by

$$
10^{-2 m / 5}=\phi / \phi_{c} \quad 10^{-2 M / 5}=\left(L_{o} / 4 \pi 10^{-10}\right) / \phi_{0}
$$

where $\phi_{0}\left(\operatorname{erg} / \sec (M p c)^{2}\right)$ is a standard flux, we may write (11) in either of the forms

$$
\begin{gathered}
\mathrm{m}=M+25+5 \log S+5(\log \varepsilon)\left\{\frac{1}{2} \ln (1+z)+\ln \ln (1+z)\right\} \\
\mathrm{m}=M+25+5 \log S-5 \log \log \varepsilon+5\left\{\frac{1}{2} \log (1+z)+\log \log (1+z)\right\}
\end{gathered}
$$


It is customary to replace the variable $z$ by $w=\log k z, k=3 \times 10^{5}$, in terms of which one may verify from (13) that

$$
\mathrm{dm} / \mathrm{dw}=(\mathrm{dm} / \mathrm{dz}) /(\mathrm{dw} / \mathrm{dz})=5\left\{\frac{1}{2} \frac{z}{1+z}+\frac{z}{(1+z) \ln (1+z)}\right\} \leq 5
$$

with limit 5 as $z \rightarrow 0$. Similarly.

$$
\mathrm{d}^{2} \mathrm{~m} / \mathrm{dw} w^{2}=\frac{5}{\log \varepsilon}\left\{\frac{z}{2(1+z)^{2}}-\frac{z[z-\ln (1+z)]}{(1+z)^{2} \ln ^{2}(1+z)}\right\} \leq 0
$$

with corresponding limit 0 .

In reality, a red shift $z \simeq \mathrm{Hs} / \mathrm{c} \equiv \mathrm{s} / \mathrm{S}$ is observed in light from galaxies at distances $s \ll S$ (Hubble's law), where $H=3.24 \times 10^{-18} \mathrm{sec}^{-1}$ is "Hubble's constant," and $S \equiv c / H=3000 \mathrm{Mpc}$.

Taking $S=3000 \mathrm{Mpc}$ in (14) and (10) gives

$$
\begin{aligned}
& m=M+44.20+5\left\{\frac{1}{2} \log (1+z)+\log \log (1+z)\right\} \\
& s=2.303(3000) \log (1+z) \quad M p c
\end{aligned}
$$

Thus, in a static universe with a friction $F=-H P\left(H=3.24 \times 10^{-18} \mathrm{sec}^{-1}\right)$ acting on photons, (15) relates the observables $m, z$, for a source of absolute magnitude $M$, while (16) gives the (unobservable) distance to the source.

It is curious that the apparent slope limits of the observed function $m(w)$ are those noted above for the function in (15), and moreover, (15) with $M=-20.3$ is in fair agreement with the observations, considering the inaccuracies in M and S. (M. L. Humason et al., Astron. J. 61. (1956) p. 149.) 
(For an "average" galaxy, $L_{0}=10^{10} L_{\odot}=4 \times 10^{43} \mathrm{erg} / \mathrm{sec}, M=-20.3$, )

The equations (15) and (16) may be used to give the absolute magnitude and distance in terms of an observed $m$ and $z$. Thus the quasar 3C9 (reported $\mathrm{m}=18.2, z=2.012$ ) would have $M=-25.6, \mathrm{~s}=3308 \mathrm{Mpc}$, while for quasar PKS $0237(m=16.63, z=2.223), M=-27.4, s=3512 \mathrm{Mpc}$.

Note 1. (Olbers' paradox and friction) Suppose infinite Euclidean space has a uniform density of $n_{0}$ motionless point galaxies per $\mathrm{cm}^{3}$, each of luminosity $L_{0}=N_{0} E_{0}$ erg/sec. Such a galaxy, at distance $s(\mathrm{~cm})$ from earth, produces a flux $\phi=L_{0} / 4 \pi \mathrm{s}^{2} \mathrm{erg} / \mathrm{sec} \mathrm{cm}^{2}$, in the simplest model, of which the earth, of radius $r$, receives an energy $\pi r^{2}$ times this. Multiplying by the nurnber $n_{0}\left(4 \pi s^{2} d s\right)$ of galaxies in the "s-shell" about earth, integration on $r<s<\infty$, and division by the earth's surface area $4 \pi r^{2}$, gives the infinite result

$$
\phi_{e}=\int_{r}^{\infty} \frac{1}{4} n_{0} L_{0} d s \quad \text { erg/sec } \operatorname{cn}^{2}
$$

for the earth's surface flux (Olbers' paradox).

Assuming the "friction" $F=-H P$ of Case 2 zbove, we should replace the above flux $\phi$ by $L_{0} e^{-s / S} / 4 \pi s^{2}(S \equiv c / H)$, obtaining the finite result

$$
\phi_{\epsilon}=\int_{r}^{\infty} \frac{1}{4} n_{0} L_{0} e^{-s / s} d s \simeq \frac{1}{4} n_{0} L_{o} s \text { erg/sec } \mathrm{cm}^{2}
$$

With $\mathrm{n}_{\mathrm{o}}=10^{-75}$ galaxies $/ \mathrm{cm}^{3}, L_{0}=4 \times 10^{43} \mathrm{erg} / \mathrm{sec}(M=-20.3)$, and $\mathrm{S}=.93 \times 10^{28} \mathrm{~cm}$, one finds $\phi_{\mathrm{e}} \simeq .93 \times 10^{-4} \mathrm{erg} / \mathrm{sec} \mathrm{cm}^{2}$, which is far too large. (Just visible flux $\phi=10^{-7} \mathrm{erg} / \mathrm{sec} \mathrm{cm}^{2}, m=6$. ) 
5. The Lorentz Transformation. Up to this point, we have considered particles and events in a single inertial frame $\sum$ of events $(R, t)$. Now suppose $\Sigma$ and $\Sigma^{\prime}$ are two such frames, each subject to the provisos of $\xi 1$, the position space of $\Sigma$ ' having constant velocity $v_{0}=v_{0} \Psi_{0}, v_{0}=\left|v_{0}\right|<c$, relative to that of $\Sigma$. We use throughout the notations

$$
\beta_{0}=v_{0} / c<1, \quad \gamma_{0}=\left(1-\beta_{0}^{2}\right)^{-\frac{1}{2}} \geq 1
$$

The Lorentz transformation then sets up a one-one correspondence

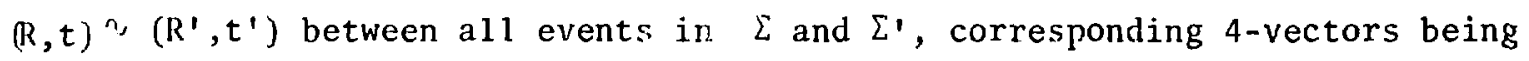
regarded as the "same event," as it appears in the two frames.

Adopting (paralle1 spatial) standard axes $S, S^{\prime}$ (Fig. 1), having coincident $x, X^{\prime}$ axes, with $\Psi_{0}=(1,0,0)_{S}$, and agreeing that the events $(0,0)_{\Sigma}$ and $(0,0)_{\Sigma}$, correspond, the Lorentz transformation assumes the simple form

$$
\begin{aligned}
& x=\gamma_{0}\left(x^{\prime}+v_{0} t^{\prime}\right) \\
& y=y^{\prime} \\
& z=z^{\prime} \\
& t=\gamma_{0}\left(\frac{v_{0}}{c^{2}} x^{\prime}+t^{\prime}\right)
\end{aligned}
$$

which is a linear transformation of determinant

$$
\gamma_{0}^{2}\left(1-\beta_{0}^{2}\right)=1
$$

Its mathematical inverse (and that of other transformations derived from it) 


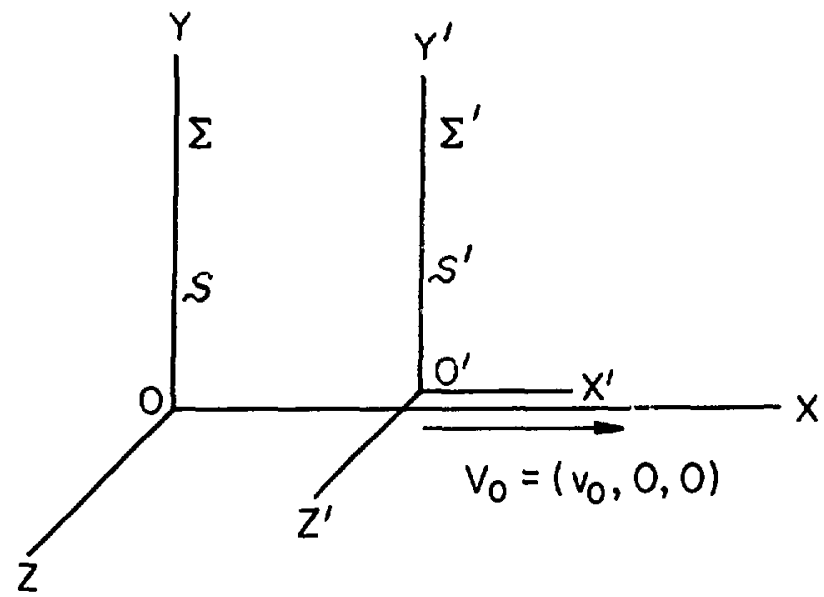

Fig. 1 .

Standard Axes 
is obtained by changing $v_{0}$ to $-v_{0}$ and interchanging primed and unprimed variables.

If we intraduce the variables $\tau=c t, \tau^{\prime}=c t^{\prime}$, we can write (2) in the more elegant form

$$
\begin{aligned}
& x=\gamma_{0}\left(x^{\prime}+\beta_{0} \tau^{\prime}\right) \\
& y=y^{\prime} \\
& z=z^{\prime} \\
& \tau=\gamma_{0}\left(\beta_{0} x^{\prime}+\tau^{\prime}\right)
\end{aligned}
$$

Two mathematical properties of the transformation (matrix) itself are easily verified, namely

$$
\begin{aligned}
& R^{2}-\tau^{2}=R^{\prime 2}-\tau^{2} \\
& |R| \leq \tau \text { and } \tau>0 \text { iff }\left|R^{\prime}\right| \leq \tau^{\prime} \quad \tau^{\prime}>0 .
\end{aligned}
$$

The first is an obvious consequence of (4). As for (6), it suffices to note that $\left|R^{\prime}\right| \leq \tau^{\prime}$ implies $|R| \leq \tau$ by (5), and moreover that $\tau^{\prime}>0$ then implies

$$
\tau=\gamma_{0}\left(\beta_{0} x^{\prime}+\tau^{\prime}\right) \geq \gamma_{0}\left(-\beta_{0}\left|R^{\prime}\right|+\tau^{\prime}\right) \geq \gamma_{0}\left(-\beta_{0} \tau^{\prime}+\tau^{\prime}\right)=\gamma_{0}\left(1-\beta_{0}\right) \tau^{\prime}>0
$$

The inverse transformation yields the converse. 
In practice, given spatial axes are usually not in the standard configuration of Fig. 1, and auxiliary rotations are required to apply the simple Lorentz transformations. These are discussed in App. I, and will be referred to when necessary. An alternative device is afforded by the "vector form" of the transformations. These are included in the appropriate sections.

The space-time Lorentz tranisformation may be written in vector form, applicable to any set of parallel spatial axes $A, A^{\prime}$ (Fig. 2). We first decompose the vectors $R, R^{\prime}$ into components parallel (/I) and perpendicular (1) to the (unit) direction vector $\Psi_{0}$ in the usual way (Fig. 3),

$$
\begin{aligned}
& R=R_{\|}+R_{\rfloor}, \quad R_{\|} \equiv\left(R \cdot \Psi_{0}\right) \Psi_{0}^{\prime}, \quad R_{\perp} \equiv R-R \| \\
& R^{\prime}=R_{\mid}^{\prime}+R_{1}^{\prime}, \quad R_{||}^{\prime}=\left(R^{\prime} \cdot \Psi_{0}\right) \Psi_{0}, \quad R_{I}^{\prime}=R^{\prime}-R_{\mid}^{\prime}
\end{aligned}
$$

The transformation (4) then asserts that

$$
\begin{aligned}
\left(R \cdot \Psi_{0}\right) & =\gamma_{0}\left(\left(K^{\prime} \cdot \Psi_{0}\right)+B_{0} \tau '\right) \\
R_{L} & =R_{j}^{\prime} \\
\tau & =\gamma_{0}\left(B_{0}\left(R^{\prime} \cdot \Psi_{0}\right)+\tau '\right)
\end{aligned}
$$

\section{Therefore}

$$
\begin{aligned}
R & =\left(R \cdot \Psi_{0}\right) \Psi_{0}+R_{\perp}=\gamma_{0}\left(\left(R^{\prime} \cdot \Psi_{0}\right)+B_{0} \tau^{\prime}\right) \Psi_{0}+R^{\prime} \\
& =R^{\prime}+\left\{\left(\gamma_{0}-1\right)\left(R^{\prime} \cdot \Psi_{0}\right)+\gamma_{0} B_{0} \tau^{\prime}\right\} \Psi_{0}
\end{aligned}
$$




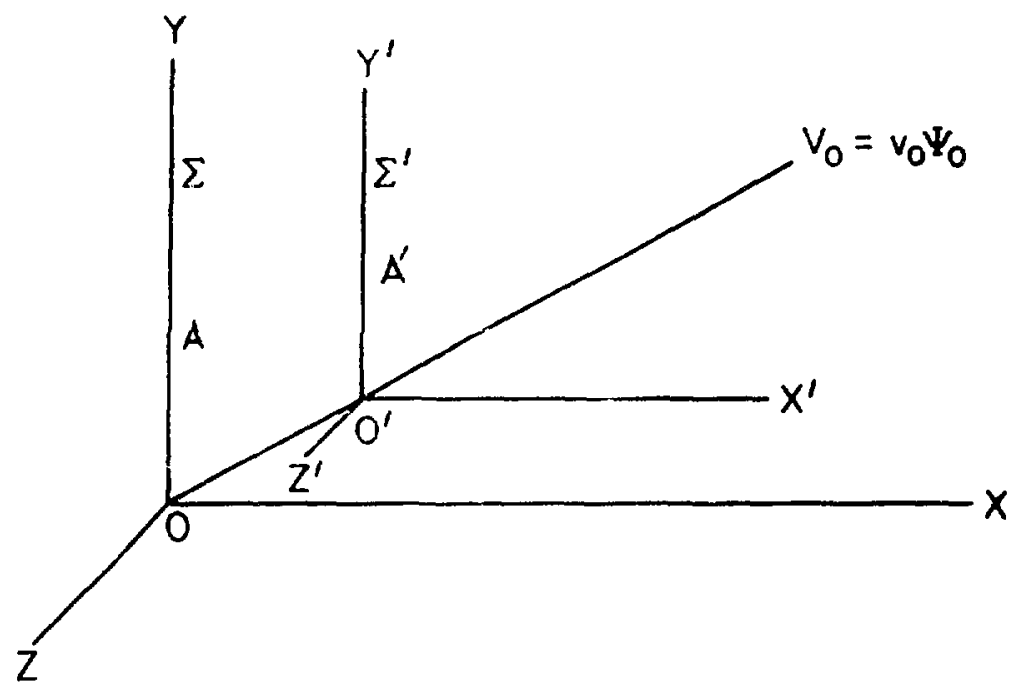

Fig. 2 .

Gencral Parallel Axes

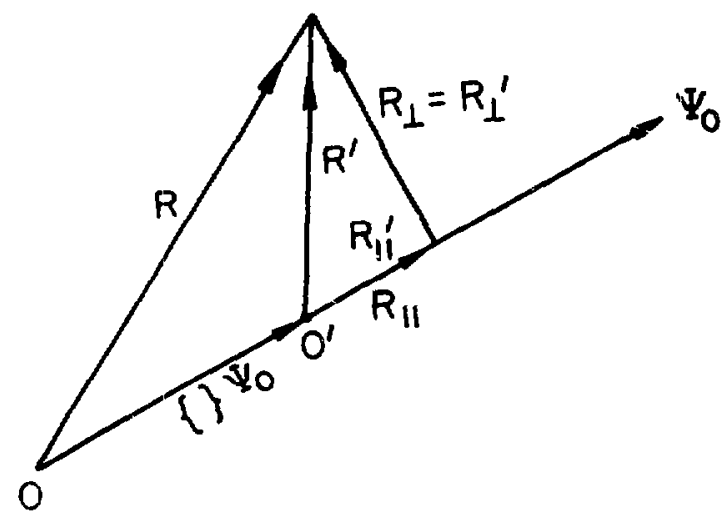

Fig. 3.

Decomposition of $R, R^{\prime}$ 
Thus we have the parallel axis Lorentz transformation

$$
\begin{aligned}
& R=R^{\prime}+\left\{\delta_{0}\left(R^{\prime} \cdot \Psi_{0}\right)+\gamma_{0} \beta_{0} \tau^{\prime}\right\} \Psi_{0}, \quad \delta_{0} \equiv\left(\gamma_{0}-1\right) \\
& \tau=\gamma_{0}\left\{\beta_{0}\left(R^{\prime} \cdot \Psi_{0}\right)+\tau^{\prime}\right\}
\end{aligned}
$$

where

$$
\begin{aligned}
R & =(x, y, z)_{A^{\prime}} \quad R^{\prime}=\left(x^{\prime}, y^{\prime}, z^{\prime}\right)_{A^{\prime}} \quad \text { and } \\
\Psi_{0} & =\left(a_{x}^{0}, a_{y}^{0}, a_{z}^{0}\right)_{A} .
\end{aligned}
$$

For some purposes it is convenient to introduce the dimensionless "velocity" $\bar{v}_{0}=\beta_{0} \Psi_{0}$ of the $\Sigma$ ' frame, and to write (7) in the form

$$
\begin{aligned}
& R=R^{\prime}+\left\{\Gamma_{0}\left(R^{\prime} \cdot \bar{V}_{0}\right)+\gamma_{0} \tau^{\prime}\right\} \bar{V}_{0} \\
& \tau=\gamma_{0}\left\{\left(R^{\prime} \cdot \bar{V}_{0}\right)+\tau^{\prime}\right\}
\end{aligned}
$$

where

$$
\Gamma_{0}=\delta_{0} / \beta_{0}^{2}=\left(\gamma_{0}-1\right) /\left(1-\gamma_{0}^{-2}\right)=\gamma_{0}^{2} /\left(\gamma_{0}+1\right)
$$

Note 1. In writing the transformation (2) from $\Sigma^{\prime}$ to $\Sigma$, it is tacitly assumed that the constant $c$, occurring explicitly, and implicitly in $\gamma_{0}$, represents the speed limit of $\xi 1$ as it appears in $\Sigma$. Due to the physically necessary condition that the mathematical inverse of (2) and the Lorentz transformation from $\Sigma$ to $\Sigma$ ' should agree, it is easy to show that the speed limit $c$ must be identical in both frames, a fundamental postulate of relativity. 
Note 2. From (5) it appears that $\left|R^{\prime}\right|=c\left|t^{\prime}\right|$ iff $|R|=c|t|$, picturesquely, "the Lorentz transformation takes the light cone into the light cone." This may be regarded as the basic feature of the transformation. Indeed, it is well known that an arbitrary non-singular linear transformation of two 4-spaces with this formal property assumes the simple form when spatial axes are p:operly aligned by rotations and units suitably standardized. For a generalization to m-space, see App. III.

6. Time dilatation. Consider a pair of corresponding events

$$
\left(R_{1}, t_{1}\right) \sim\left(\hat{R}^{\prime}, t_{1}^{\prime}\right), \quad\left(R_{2}, t_{2}\right) \sim\left(\hat{R}^{\prime}, t_{2}^{\prime}\right)
$$

occurring at the same point $\hat{R}^{\prime}$ of $\Sigma^{\prime}$. From (5.4) we obtain

$$
\begin{aligned}
& x_{2}-x_{1}=\gamma_{0} \beta_{0}\left(\tau_{2}^{\prime}-\tau_{1}^{\prime}\right) \\
& y_{2}-y_{1}=0 \\
& z_{2}-z_{1}=0 \\
& \tau_{2}-\tau_{1}=\gamma_{0}\left(\tau_{2}^{\prime}-\tau_{1}^{\prime}\right)
\end{aligned}
$$

Thus $x_{2}-x_{1}=\beta_{0}\left(\tau_{2}-\tau_{1}\right)=v_{0}\left(t_{2}-t_{1}\right)$, as is to be expected, but we have also the time dilatation effect

$$
\tau_{2}-\tau_{1}=\gamma_{0}\left(\tau_{2}^{\prime}-\tau_{1}^{\prime}\right)>\tau_{2}^{\prime}-\tau_{1}^{\prime}
$$

which states that "a moving clock appears to run slow." 
It follows that a free material particle, fixed at $\hat{R}^{\prime}$ in its own rest frame $\hat{\Sigma}^{\prime}$, with an intrinsic life span $T^{\prime}$, and moving with constant velocity $v_{0}$ in $\Sigma$, appears in the latter frame to have a life span $T$ and to travel a distance $D$ given by

$$
T=Y_{0} T^{\prime}>T^{\prime}, \quad D=\gamma_{0} B_{0} c T^{\prime}=B_{0} c T
$$

Note here the identity of the transformation parameters with those of the particle in $\Sigma$, and the significant relation

$$
\mathrm{T} / \mathrm{T}^{\prime}=\gamma_{0}=\gamma=\mathrm{E} / \mathrm{e}
$$

(picturesquely, energy in $\Sigma$ is the secret of longevity in $\Sigma$.)

Note 1. A free $5 \mathrm{MeV} \pi^{+}$particle $(e=140 \mathrm{MeV})$ of intrinsic life span $T^{\prime}=2.6: 10^{-S} \mathrm{sec}$ travels $210 \mathrm{~cm}$ in a $\Sigma$ life span of $\mathrm{T}=2.7 \times 10^{-8} \mathrm{sec}$. At $500 \mathrm{MeV}$ it could attain $3480 \mathrm{~cm}$ in $\mathrm{T}=11.9 \times 10^{-8} \mathrm{sec} .\left(\gamma_{0}=1+\mathrm{k} / \mathrm{e}\right.$, $\left.\gamma_{0} \beta_{0}=\left(\gamma_{0}^{2}-1\right)^{\frac{1}{2}}\right)$

These $\sum$ life spans may be compared with the travel times of Note 3.1 , where $k=5 \mathrm{MeV}$ was terminal, and Note 3.5, where $k=500 \mathrm{MeV}$ was constant. Note 2. Strictly speaking, special relativity does not give the $\Sigma$ life span of a particle moving with non-constant velocity $\mathrm{V}$. However, integration of $d \tau^{\prime}=d \tau / Y(\tau)$, the instantaneous version of $(1)$, leads to the formula

$$
\tau^{\prime}=\int_{0}^{\tau} \mathrm{d} \tau / \gamma(\tau)
$$

With $\tau^{\prime}=c T^{\prime}, \tau=c T$, this gives a possible generalization of the relation 
$T^{\prime}=T / Y_{0}$ of (2). For the circular motion of Note 3.5, $Y$ is constant, and (4) yields the same $\Sigma$ span $T$ as does (2) for the corresponding constant velocity. However, for the accelerated 1inear motion of Note 3.1 , (4) gives

$$
\tau^{\prime}=\int_{0}^{\tau} \mathrm{d} \tau /\left(1+\varepsilon^{2} \tau^{2}\right)^{\frac{1}{2}}=\varepsilon^{-1} \ln \left(\varepsilon \tau+\left(1+\varepsilon^{2} \tau^{2}\right)^{\frac{1}{2}}\right)
$$

whence

$$
T=(1 / \varepsilon c) \sinh \left(\varepsilon c T^{\prime}\right)>T^{\prime}
$$

In the example cited, $T$ is only slightly greater than $T^{\prime}$.

Note 3. A $545 \mathrm{MeV} \equiv{ }^{0}$ particle $(e \simeq 1315 \mathrm{MeV})$ traveling $3 \mathrm{~cm}$ in its $\sum 1$ ife span, has an instrinsic life span of $10^{-10} \mathrm{sec}$. In fact

$$
T^{\prime}=D / Y_{0} B_{0} c=D / c\left(Y_{0}^{2}-1\right)^{\frac{1}{2}} \simeq 10^{-10}
$$

since

$$
\gamma_{0}=1+(k / e) \simeq 1.414 \simeq \sqrt{ } 2
$$

Note 4. An earth man with $40 Y$ to 1 ive can reach $\alpha$-Centauri, 4 LY away, at a speed of $c / \sqrt{ } 101$. If his rest mass is $10^{5}$ gm (neglecting the ship) this requires a k.e. $\simeq 500 \mathrm{c}^{2}$ erg $\simeq 9$ megatons high explosive. (1 kt H.E. $\simeq 5 \mathrm{x}$ $\left.10^{19} \mathrm{erg}\right)$. For if $Y i$ the number of seconds in a year, we have

$$
\begin{aligned}
& \left(\gamma_{0}^{2}-1\right)^{\frac{1}{2}}=\gamma_{0}^{\beta_{0}}=\mathrm{D} / \mathrm{cT}=4 \mathrm{cY} / \mathrm{c}(40 Y)=1 / 10, \quad \gamma_{0}^{2}=101 / 100, \\
& \beta_{0}=\left(1-\gamma_{0}^{-2}\right)^{\frac{1}{2}}=1 / \sqrt{101,} \quad v_{0}=c / \sqrt{ } 101, \quad k=e\left(\gamma_{0}-1\right) \simeq \mathrm{e} / 200 .
\end{aligned}
$$

Note 5. If two identical particles $A$ and $A^{\prime}$, each of intrinsic life span $T_{0}$ 
in its own rest frame $\Sigma, \Sigma^{\prime}$, are created simultaneously at $(0,0) \Sigma \sim(0,0)_{\Sigma}$, , and separate linearly with relative speed $v_{0}$, then $A$ dies before $A^{\prime}$ in $\Sigma$, but after $A^{\prime}$ in $\Sigma^{\prime}$. In fact the events of their deaths are given by (5.4) as

$$
\begin{array}{cccc} 
& \Sigma & \Sigma^{\prime} \\
D\left(A^{\prime}\right) & \left(\gamma_{0} \beta_{0} \tau_{0}, \gamma_{0} \tau_{0}\right) & \sim & \left(0, \tau_{0}\right) \\
D(A) & \left(0, \tau_{0}\right) & \sim & \left(-\gamma_{0} \beta_{0} \tau_{0}, \gamma_{0} \tau_{0}\right)
\end{array} \quad \tau_{0} \equiv \mathrm{cT}
$$

Since (5.4) is linear, this implies

$$
\left(\gamma_{0} \beta_{0} \tau_{0},\left(\gamma_{0}-1\right) \tau_{0}\right) \sim\left(\gamma_{0} \beta_{0} \tau_{0},-\left(\gamma_{0}-1\right) \tau_{0}\right)
$$

Why does this not contradict (5.6)? The situation is schematized in Fig. 1. Note 6. Another instance of the time dilatation effect is seen in the frequency of arrival of photons from a receding source. If a photon source at $0^{\prime}$ in $\Sigma^{\prime}$ emits two photons in the direction $\psi^{\prime}=(-1,0,0)_{S}$, at time $t_{i}^{\prime}<t_{2}^{\prime}$, then the $\sum$ events of emission are $x_{i}=\gamma_{0} \beta_{0} c t_{i}^{\prime}, t_{i}=\gamma_{0} t_{i}^{\prime}$, and hence the times of arriva $i$ at 0 in $\sum$ are

$$
T_{i}=t_{i}+\left(x_{i} / c\right)=\gamma_{0}\left(1+B_{0}\right) t_{i} ; \quad i=1,2
$$

with a time interval

$$
\Delta T=\gamma_{0}\left(1+\beta_{0}\right) \Delta t^{\prime} .
$$




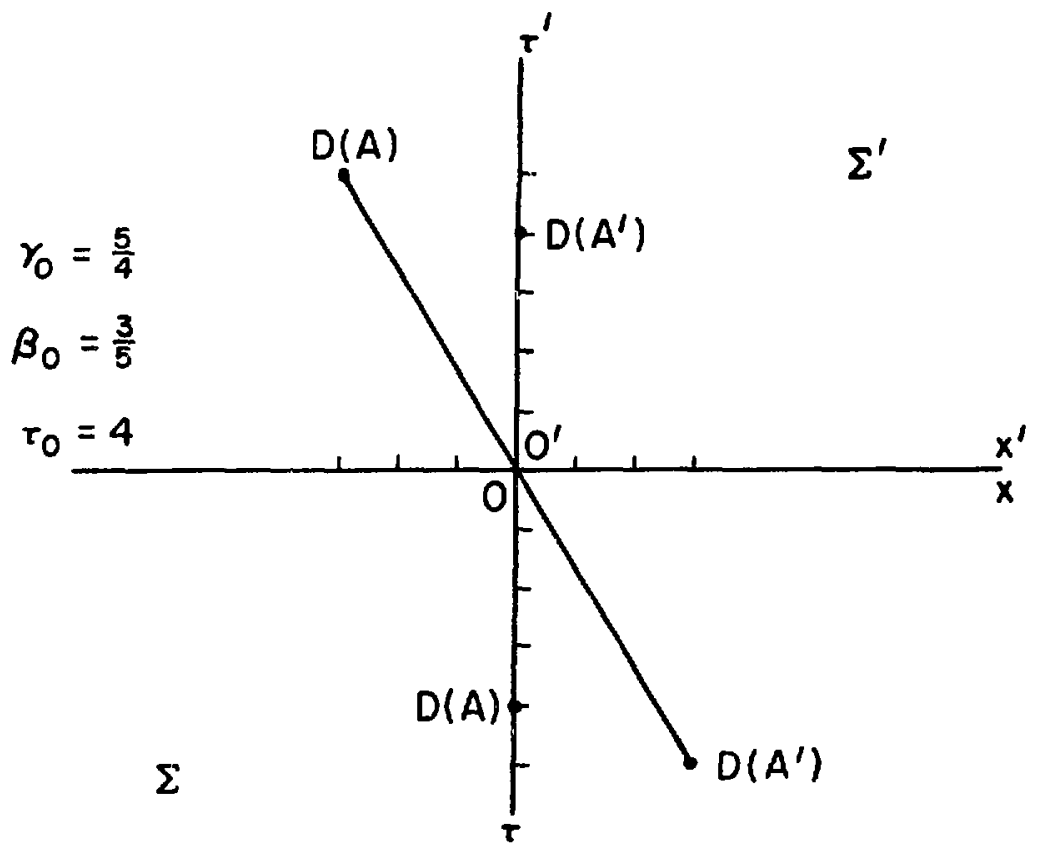

Fig. 1 .

Time Reversal 
If the source emits $N^{\prime}$ such photons per sec. in $\Sigma^{\prime}$, then the number per sec. received at 0 in $\Sigma$ is

$$
N=1 / \Delta T=1 / \gamma_{0}\left(1+\beta_{0}\right) \Delta t^{\prime}=\gamma_{0}\left(1-\beta_{0}\right) N^{\prime}<N^{\prime}
$$

This is precisely the transformation governing the energy degradation of each photon due to the Doppler effect (\$12) but is an independent additional phenomenon decreasing the energy flux at 0 in $\Sigma$.

7. Fitzgerald contraction. To emphasize the mathematical analogy with time dilatation, we consider the pair of corresponding events

$$
\left(R_{1}, t_{1}\right) \sim\left(R_{1}^{\prime}, \hat{t}^{\prime}\right), \quad\left(R_{2}, t_{2}\right) \sim\left(R_{2}^{\prime}, \hat{t}^{\prime}\right)
$$

occurring at the same time $\hat{t}^{\prime}$ in $\Sigma^{\prime}$. From $(5.4)$ we have

$$
\begin{aligned}
& x_{2}-x_{1}=\gamma_{0}\left(x_{2}^{\prime}-x_{1}^{\prime}\right) \\
& y_{2}-y_{1}=y_{2}^{\prime}-y_{1}^{\prime} \\
& z_{2}-z_{1}=z_{2}^{\prime}-z_{1}^{\prime} \\
& \tau_{2}-\tau_{1}=\gamma_{0} \beta_{0}\left(x_{2}^{\prime}-x_{1}^{\prime}\right)
\end{aligned}
$$

Hence,

$$
x_{2}-x_{1}=\gamma_{0}\left(x_{2}^{i}-x_{1}^{i}\right)>\left(x_{2}^{i}-x_{1}^{\prime}\right) \text {, or equivalent } 1 y
$$




$$
x_{2}^{\prime}-x_{1}^{\prime}=\left(x_{2}-x_{1}\right) / \gamma_{0}<x_{2}-x_{1}
$$

which embodies the Fitzgerald contraction effect.

Physically interpreted, it shows that a thin rod, rigid and fixed in its own rest frame $\Sigma$, and undergoing translation at speed $v_{0}$ in $\Gamma_{1}^{\prime}$, appears in the latter frame with dimensions

$$
\begin{aligned}
& \Delta x^{\prime}=\Delta x / \gamma_{0}<\Delta x \\
& \Delta y^{\prime}=\Delta y \\
& \Delta z^{\prime}=\Delta z
\end{aligned}
$$

where $\Delta x^{\prime}, \Delta x$ are the dimensions in the direction of motion, (standard axes!) and the ends of the rod are observed simultaneously in $\Sigma^{\prime}$.

Very roughly speaking, we may say that a moving time interval appears longer, while a moving space interval appears shorter. The apparent symmetry is imperfect in that the first statement refers to a common position in the rest frame of an observed object (clock), whereas the second refers to a common time not in the rest frame of the observed object (rod).

From (2) we may infer the transformations

$$
\begin{array}{ll}
v^{\prime}=V / \gamma_{0}<v & \mathrm{~cm}^{3} \\
n^{\prime}=n \gamma_{0}>n & \mathrm{~cm}^{-3} \\
\rho^{\prime}=\rho \gamma_{0}^{2}>\rho & \text { gm } \mathrm{cm}^{-3}
\end{array}
$$

for volume $V^{\prime}$, numerical density $n^{\prime}$, and density $\rho^{\prime}$ in terms of their proper values in their rest frame $\Sigma$. 
Note 1. A graphite $\operatorname{rod}\left(\rho=2.25 \mathrm{gm} / \mathrm{cm}^{3}, M=12\right)$ nar. numerical density $\mathrm{n}=\rho /\left(\mathrm{M} / \mathrm{N}_{\mathrm{o}}\right)=1.13 \times 10^{23} \mathrm{C}$ atoms per $\mathrm{cm}^{3}$ in its rest frame. If translated at speed $v_{0}=c / \sqrt{ } 2\left(\gamma_{0}=\sqrt{2}\right)$, its numerical density appears to be $1.6 \times 10^{23}$ (anisotropic!).

Note 2. A "car" of length $5 \mathrm{~cm}$ in its own rest frame $\Sigma$, and moving with speed $(3 / 5) c$ in $\Sigma^{\prime}\left(-X^{\prime}\right.$ direction on $\left.S^{\prime}\right)$ appears to have 1 ength $4 \mathrm{~cm}$ in $\Sigma^{\prime}$ when its ends are observed simuitaneously in $\Sigma^{\prime}$. When $t^{\prime}=5 / \mathrm{c}$ sec, it just fits into a "garage" $4 \mathrm{~cm}$ long, and the door is slammed on it. An observer in $\sum$ sees the $5 \mathrm{~cm}$ car at rest, with a $3-1 / 5 \mathrm{~cm}$ garage approaching it (ends of garage observed simultaneously in $\Sigma$ ). What happens in $\Sigma$ ? The reader may puzzle over Fig. 1, which we give without comment.

8. The velocity transformation. For trajectorjes

$$
(R(t), t) \sim\left(R^{\prime}\left(t^{\prime}\right), t^{\prime}\right)
$$

corresponding event by event in the frames $\Sigma, \Sigma^{\prime}$ of $\$ 5$, the Lorentz transformation (5.4) provides a $\tau^{\prime}$-parameterization of the trajectory $(R(t), t)$ in terms of the trajectory $\left(R^{\prime}\left(t^{\prime}\right), t^{\prime}\right)$, namely

$$
\begin{aligned}
& x=\gamma_{0}\left(x^{\prime}\left(\tau^{\prime}\right)+\beta_{0} \tau^{\prime}\right) \\
& y=y^{\prime}\left(\tau^{\prime}\right) \\
& z=z^{\prime}\left(\tau^{\prime}\right) \\
& \tau=\gamma_{0}\left(\beta_{0} x^{\prime}\left(\tau^{\prime}\right)+\tau^{\prime}\right)
\end{aligned}
$$

relative to standard axis $\mathcal{S}, \mathcal{S}^{\prime}$. 


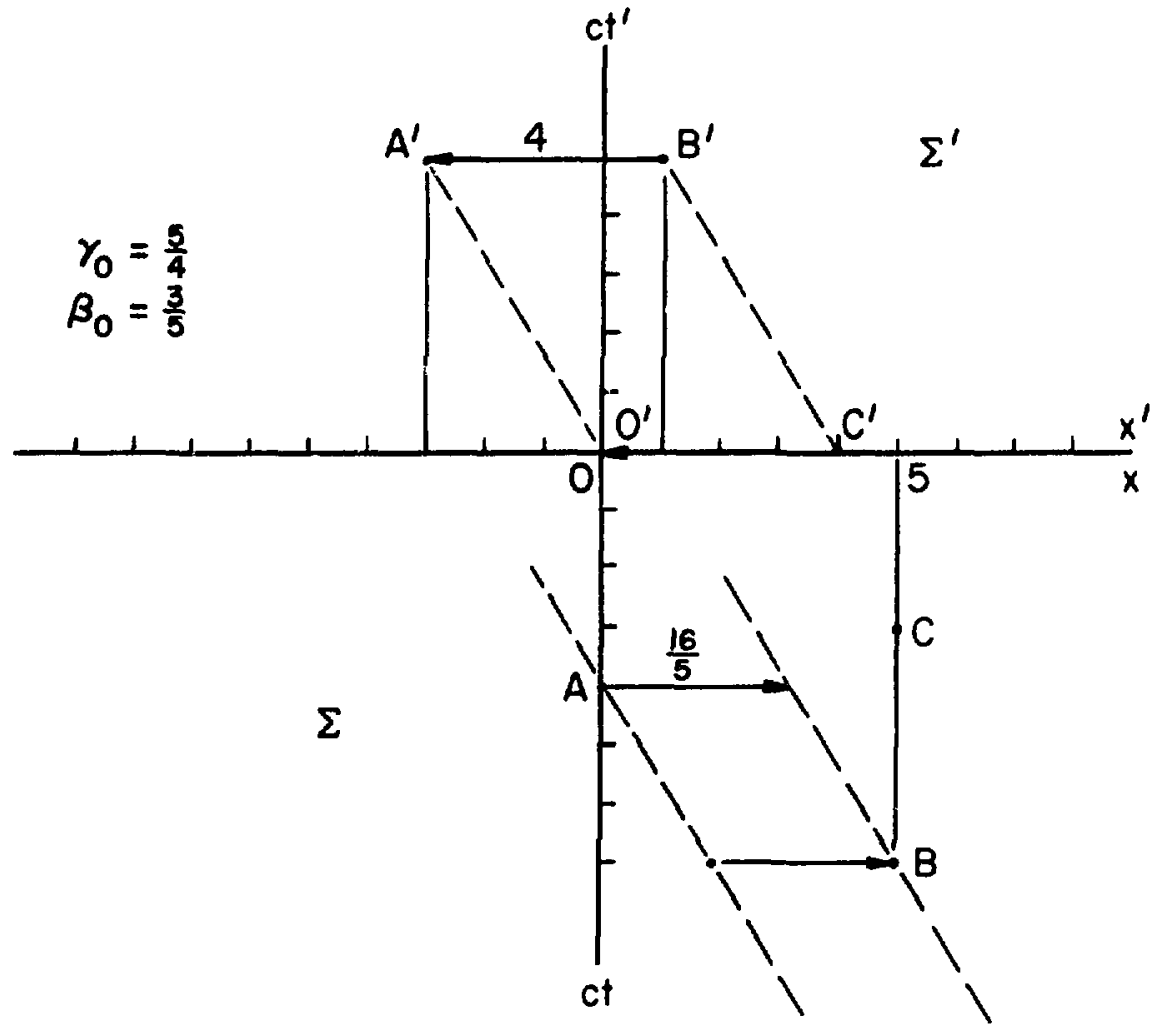

Fig. 1 .

The Car and the Garage 
It is convenient to introduce here the dimensionless velocities

$$
\begin{aligned}
& \mathrm{dR} / \mathrm{d} \tau=V / c=\left(\beta_{x}, \beta_{y}, \beta_{z}\right)=\beta \Psi=\bar{v} \\
& \mathrm{dR} \cdot / \mathrm{d} \tau^{\prime}=V^{\prime} / c=\left(\beta_{x}^{\prime}, \beta_{y}^{\prime}, \beta_{z}^{\prime}\right)=\beta^{\prime} \psi^{\prime}=\bar{V}^{\prime}
\end{aligned}
$$

Before deriving from (1) the transformation for velocities, we first obtain some preliminary results. From (1), (2), and the identity

$$
\begin{aligned}
& (\mathrm{dx} / \mathrm{d} \tau)\left(\mathrm{d} \tau / \mathrm{d} \tau^{\prime}\right)=\mathrm{dx} / \mathrm{d} \tau^{\prime} \text {, we have } \\
& \beta_{x} \cdot \gamma_{0}\left(\beta_{0} \beta_{x}^{\prime}+1\right)=\gamma_{0}\left(\beta_{x}^{\prime}+\beta_{0}\right)
\end{aligned}
$$

Hence for the important quantities

$$
d^{\prime}=1+\beta_{0} \beta_{x}^{\prime} \quad d=1-\beta_{0} \beta_{x}
$$

we see from (3) that $d^{\prime} d=d^{\prime}-\beta_{0} \beta_{x} d^{\prime}=\left(1+\beta_{0} \beta_{x}^{\prime}\right)-\beta_{0}\left(\beta_{x}^{\prime}+\beta_{0}\right)=1 / Y_{0}^{2}$, whence

$$
\left(\gamma_{0} d^{\prime}\right)\left(\gamma_{0} d\right) \equiv 1
$$

Since $d=1-\beta_{0} \beta_{x} \geq 1-\beta_{0} \beta \geq 1-\beta_{0}>0$, we have both

$$
\mathrm{d}>0 \text { and } \mathrm{d}^{\prime}>0
$$

Now from (1) and the identity $d R / d \tau=\left(d R / d \tau^{\prime}\right) /\left(d \tau / d \tau^{\prime}\right)$ we obtain the 
$\bar{V}, \bar{V}^{\prime}$ transformation on standard axes $S, S^{\prime}$ :

$$
\begin{array}{ll}
\beta_{x}=\gamma_{0}\left(\beta_{x}^{\prime}+\beta_{0}\right) / \gamma_{0} d^{\prime} & \\
\beta_{y}=\beta_{y}^{\prime} / \gamma_{0} d^{\prime} & \\
\beta_{z}=\beta_{z}^{\prime} / \gamma_{0} d^{\prime} & d^{\prime} \equiv 1+\beta_{0} \beta_{x}^{\prime}>0
\end{array}
$$

with the inverse

$$
\begin{aligned}
& \beta_{x}^{\prime}=\gamma_{0}\left(\beta_{x}-\beta_{0}\right) / \gamma_{0} d \\
& \beta_{y}^{\prime}=\beta_{y} / \gamma_{0} d \\
& \beta_{z}^{\prime}=\beta_{z} / \gamma_{0} d \quad d \equiv 1-\beta_{0} \beta_{x}>0
\end{aligned}
$$

From (7), we compute $\beta_{x}^{2}+\beta_{y}^{2}+\beta_{z}^{2}-1=\left\{\gamma_{0}^{2}\left(\beta_{x}^{\prime}+\beta_{0}\right)^{2}+\beta_{y}{ }^{2}+\beta_{z}^{\prime 2}-\gamma_{0}^{2}\left(1+\beta_{0} \beta_{x}^{\prime}\right)^{2}\right\} / \gamma_{0}^{2} d^{2}$

$=\left\{\gamma_{0}^{2}\left(1-\beta_{0}^{2}\right) \beta_{x}^{\prime 2}+\beta_{y}^{\prime 2}+\beta_{z}^{\prime 2}-\gamma_{0}^{2}\left(1-\beta_{0}^{2}\right)\right\} / \gamma_{0}^{2} d^{\prime 2}$

which shows that

$$
\beta_{x}^{2}+\beta_{y}^{2}+\beta_{z}^{2}-1=\left(\beta_{x}^{\prime 2}+\beta_{y}^{\prime 2}+\beta_{z}^{\prime 2}-1\right) / Y_{o}^{2} d^{\prime 2}
$$

or

$$
1-\beta^{2}=\left(1-\beta^{\prime 2}\right) / \gamma_{0}^{2} d^{2}
$$


This is in effect the transformation 1 aw for speed. Since $B=v / c$, $B^{\prime}=v^{\prime} / c$, this points up the fact that the speed limit $c$ must be the same in $\Sigma$ and $\Sigma^{\prime}$. Moreover, $v=c$ iff $v^{\prime}=c$, so that an immaterial particle in $\Sigma^{\prime}$ must so appear in $\Sigma$, i.e.,

$$
m=0=m^{\prime}
$$

For a material particle, with $B^{\prime}<1, B^{\prime}<1$, we see from (10) that the $\gamma, \gamma^{\prime}$ transformation is

$$
\gamma=\gamma^{\prime} \gamma_{0} d^{\prime}
$$

where we recall the definition

$$
d^{\prime}=1+\beta_{0} \beta_{x}^{\prime}=1+\beta_{0}\left(\overline{V^{\prime}} \cdot \Psi_{0}\right)
$$

If we agree that rest mass $m>0$ is invariant:

$$
\mathrm{m}=\mathrm{m}^{\prime}>0 \quad \mathrm{e}=\mathrm{e}^{\prime}>0
$$

then we may infer from (12) the mass and energy transformations

$$
M=M^{\prime} \gamma_{0} d^{\prime}, \quad E=E^{\prime} \gamma_{0} d^{\prime} \quad(m>0)
$$

for a material particle. 
By the same method we may derive from (5.7) the vector form of the $\bar{V}, \bar{V}^{\prime}$ transformation on the parallel axes $A, A^{\prime}$, namely

$$
\begin{aligned}
& \bar{V}=\left(\gamma_{0} d^{\prime}\right)^{-1}\left[\overline{V^{\prime}}+\left\{\delta_{0}\left(\bar{V} \cdot \cdot \Psi_{0}\right]+\gamma_{0} \beta_{0}\right\} \Psi_{0}\right] \\
& d^{\prime} \equiv\left\{1+\beta_{0}\left(\bar{V} \cdot \cdot \Psi_{0}\right)\right\}, \quad \delta_{0} \equiv \gamma_{0}-1
\end{aligned}
$$

where

$$
\bar{V}=\left(B_{x}, B_{y}, B_{z}\right)_{A} \quad \bar{V}^{\prime}=\left(B_{x}^{\prime}, B_{y}^{\prime}, B_{z}^{\prime}\right)_{A^{\prime}}
$$

Again, with $\bar{V}_{0}=B_{0} \Psi_{0}$, this may be written as

$$
\begin{aligned}
& \bar{V}=\left(\gamma_{0} d^{\prime}\right)^{-1}\left[\bar{V}^{\prime}+\left\{\Gamma_{0}\left(\bar{V}^{\prime} \cdot \bar{V}_{0}\right)+\gamma_{0}\right\} \bar{V}_{0}\right] \\
& d^{\prime}=\left\{1+\left(\bar{V}^{\prime} \cdot \cdot \bar{V}_{0}\right)\right\} \quad \Gamma_{0}=\gamma_{0}^{2} /\left(\gamma_{0}+1\right)
\end{aligned}
$$

where

$$
\vec{v}_{0}=\left(B_{x}^{0}, B_{y}^{0}, B_{z}^{0}\right)_{A}
$$

Example 1. A particle of rest energy 4 moves with speed $(5 / 13) \mathrm{c}$ at $60^{\circ}$ with the $O X^{\prime}$ axis of $\Sigma^{\prime}$, while $0 X^{\prime}$ travels on $0 x$ in $\Sigma$ with speed (3/5)c. Then

$$
\begin{aligned}
& B_{0}=3 / 5, \quad \gamma_{0}=5 / 4, \\
& B^{\prime}=5 / 13, \quad \gamma^{\prime}=13 / 12, \quad E^{\prime}=\mathrm{e} \gamma^{\prime}=4(13 / 12)=13 / 3, \text { and } \\
& E=E^{\prime} \gamma_{0}\left(1+\beta_{0} \beta^{\prime} \cos \psi^{\prime}\right)=(13 / 3)(5 / 4)(1+(3 / 5)(5 / 13)(1 / 2))=145 / 24
\end{aligned}
$$


If it travels at $180^{\circ}$ with $O x^{\prime}$ with speed $(3 / 5) c$ then $E=4$. Why:

9. The direction transformation. At corresponding events on the trajectories of 58 , we have

$$
\bar{V}=B^{\prime} \sim \bar{V}^{\prime}=B^{\prime} \Psi^{\prime} \quad\left(B B^{\prime} \neq 0\right)
$$

where $\Psi, \Psi \cdot$ are the directions of the particle as they appear in the two frames. The $\bar{v}, \bar{v}$ ' transformation (8.7) therefore yields the $\Psi, \Psi$ ' transformation on standard axes:

$$
\begin{aligned}
& a_{x}=\gamma_{0}\left(a_{x}^{\prime}+\rho^{\prime}\right) / \gamma_{0} D^{\prime}, \quad \rho^{\prime} \equiv \beta_{0} / B^{\prime} \\
& a_{y}=a_{y}^{\prime} / \gamma_{0} D^{\prime} \\
& a_{z}=a_{z}^{\prime} / \gamma_{0} D^{\prime}, \quad D^{\prime} \equiv d^{\prime} B / B^{\prime}=\left\{\left(a_{x}^{\prime}+\rho^{\prime}\right)^{2}+\gamma_{0}^{-2}\left(1-a_{x}^{\prime 2}\right)\right\}^{\frac{1}{2}}
\end{aligned}
$$

The above evaluation of $D^{\prime} \equiv \mathrm{d}^{\prime} B / \beta^{\prime}$ may be verified using (8.7) thus:

$$
\begin{aligned}
& \gamma_{0}^{2} d^{\prime}{ }^{2} \beta^{2}=\gamma_{o}^{2} d^{\prime}{ }^{2}\left(\beta_{x}^{2}+\beta_{y}^{2}+\beta_{z}^{2}\right)=\gamma_{0}^{2}\left(\beta_{x}^{p}+\beta_{o}\right)^{2}+\beta_{y}^{2}+\beta_{z}^{\prime 2} \\
& =\gamma_{0}^{2}\left(\beta_{x}^{\prime}+\beta_{0}\right)^{2}+\left(\beta^{\prime 2}-\beta_{x}^{2}\right) . \text { Therefore } \\
& D^{\prime 2}=d^{\prime 2} \beta^{2} / \beta^{\prime 2}=\left(a_{x}^{\prime}+\left(\beta_{0} / \beta^{\prime}\right)\right)^{2}+\gamma_{0}^{-2}\left(1-a_{x}^{\prime 2}\right)
\end{aligned}
$$

In like fashion, one obtains from (8.8) the inverse transformation 


$$
\begin{aligned}
& \left.a_{x}^{\prime}=y_{0}\left(a_{x}-\left(B_{0} / \theta\right)\right) / r_{0} 0\right) \\
& \left.a_{y}^{\prime}=a_{y} / y_{0}\right) \\
& \left.a_{z}^{\prime}=a_{z} / r_{0}\right) \quad \quad b=\left(\left(a_{x}-\left(b_{0} /(b)\right)^{2}+y_{0}^{-2}\left(1-a_{x}^{2}\right)\right)^{\frac{1}{2}}\right.
\end{aligned}
$$

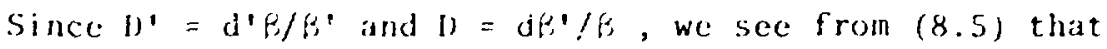

$$
\left.\left(r_{0}(1)\right)\left(\gamma_{0} l\right)\right)=1
$$

By differentiating the first equation of $(2)$, with $\ell^{\prime}=|\bar{v}|$ fixed, we obtain

$$
\mathrm{da}_{x} / \mathrm{da} a_{x}^{\prime}=\left(1+p^{\prime} a_{x}^{\prime}\right) / \gamma_{0}^{2} b^{3}
$$

a relation required in transforming differential cross sections.

For an immaterial particle, we have $\beta=1=B^{\prime}, D^{\prime}=d^{\prime}=1+B_{0} a^{\prime}$, and (2), (5) become simply

$$
\begin{aligned}
& a_{x}=\gamma_{0}\left(a_{x}^{\prime}+\beta_{0}\right) / \gamma_{0} d^{\prime} \\
& a_{y}=a_{y}^{\prime} / \gamma_{0} d^{\prime} \\
& a_{z}=a_{z}^{\prime} / \gamma_{0} d^{\prime} \quad d^{\prime}=1+\beta_{0} a_{x}^{\prime} \quad(m=0) \\
& d a_{x} / d a_{x}^{\prime}=1 / \gamma_{0}^{2} d^{\prime} \quad \quad(m=0)
\end{aligned}
$$

The vector form of the $\Psi, \Psi^{\prime}$ transformation on parallel axes $A, A^{\prime}$ appears from $(8.16)$ to be 


$$
\begin{aligned}
& \psi=\left(\gamma_{0} D^{\prime}\right)^{-1}\left[\Psi^{\prime}+\left\{\delta_{0}\left(\Psi^{\prime} \cdot \psi_{0}^{\prime}\right)+\gamma_{0} \rho^{\prime}\right\} \psi_{0}\right], \quad n^{\prime}=\beta_{0} / B^{\prime} \\
& \eta^{\prime}=\left[\left(\left[y^{\prime} \cdot \psi_{0}\right)+w^{\prime}\right]^{2}+\gamma_{0}^{-2}\left[1-\left(\psi^{\prime} \cdot \psi_{0}^{\prime}\right)^{2}\right]\right\}^{\prime 2}=\mathrm{d} \cdot \beta / \beta^{\prime} \\
& d^{\prime}=1+z_{0}^{\prime} \prime^{\prime}\left(y^{\prime} \cdot \psi^{\prime}{ }_{0}\right)
\end{aligned}
$$

Setting $\sigma=\nabla^{\prime}=1$ gibes the trancformation for an immaterial particle:

$$
\begin{aligned}
& \gamma=\left(\gamma_{0} d^{\prime}\right)^{-1}\left[\varphi^{\prime}+\left\{\delta_{0}\left(\psi^{\prime} \cdot \psi_{0}\right)+\gamma_{0} \beta \beta_{0}\right\} \Psi_{0}\right] \\
& d^{\prime}=1+P_{0}\left(w^{\prime} \cdot \Psi_{0}\right) \quad(m=0)
\end{aligned}
$$

10. Transformation of the energy 4-vector (cP, E). We have seen in $\$ 8$ that the kinematics of the Lorentz transformation, together with the assumption of rest mass invariance, implies the energy transformation

$$
E=E^{\prime} \gamma_{0} d^{\prime}, \quad d^{\prime} \equiv\left(1+\beta_{0} \beta_{x}^{\prime}\right)
$$

for a naterial particle. Since the energy of an immaterial particle is an independent parameter, it is clear that no such transformation can be derived from the space-time Lorentz transformation, and we shall take (1) as axiomatic for all particles (which implies the invariance $m=m$ ' for material particles in view of $(8.12))$.

If we now observe that $c P=c P \Psi=E B \Psi=E \bar{V}$, then (1) and the $\bar{V}, \bar{V}$ ' transformation $(8,7)$ yield the transformation for the energy 4-vector (cP,E) ح ( $\left(P^{\prime}, E^{\prime}\right)$, which is not only linear, but has the same matrix as the Lorentz transformation itself, namely 


$$
\begin{aligned}
& c p_{x}=\gamma_{0}\left(C P_{x}^{\prime}+B_{0} E^{\prime}\right) \\
& c p_{y}=c p_{y}^{\prime} \\
& c p_{z}=c p_{z}^{\prime} \\
& E=\gamma_{0}\left(E_{0} c P_{x}^{\prime}+E^{\prime}\right)
\end{aligned}
$$

on standard axes $S, S^{\prime}$. For example, $c P_{x}=E B_{x}=E^{\prime} \gamma_{0} d^{\prime} B_{x}=E^{\prime} \gamma_{0}\left(B_{x}^{\prime}+B_{0}\right)$

$=\gamma_{0}\left(C P_{x}^{\prime}+B_{0} E^{\prime}\right)$, and $E=E^{\prime} \gamma_{0} d^{\prime}=E^{\prime} \gamma_{0}\left(B_{0} B_{x}^{\prime}+1\right)=\gamma_{0}\left(E_{0} c P_{x}^{\prime}+E^{\prime}\right)$

Referring to (5.5) and (5.6), we see that

$$
\begin{aligned}
& (c p)^{2}-E^{2}=\left(c p^{\prime}\right)^{2}-E^{\prime 2} \\
& c p \leq E \text { and } E>0 \text { iff } c p^{\prime} \leq E^{\prime} \text { and } E^{\prime}>0
\end{aligned}
$$

From these we may infer the invariance of proper energy

$$
e=e^{\prime}
$$

and the preservation under (2) of the validity condition (1.3) which reads

$$
E>0 \text { and }(c P)^{2}=E^{2}-e^{2}
$$

in terms of energy parameters $(c P, E, e)$.

The vector form of (2) on the axis $A, A^{\prime}$ is of course

$$
\begin{aligned}
c P & =c P^{\prime}+\left\{\delta_{0}\left(c P^{\prime} \cdot \Psi_{0}\right)+Y_{0} \beta_{0} E^{\prime}\right\} \Psi_{0}, \quad \delta_{0} \equiv \gamma_{0}-1 \\
E & =\gamma_{0}\left\{\beta_{0}\left(c P^{\prime} \cdot \Psi_{0}\right\}+E^{\prime}\right\}
\end{aligned}
$$


With $\bar{V}_{0}=B_{0} \Psi_{0}$, this becomes

$$
\begin{aligned}
& c P=c P^{\prime}+\left\{\Gamma_{0}\left(c^{\prime} \cdot \cdot \bar{V}_{0}\right)+\gamma_{0} E^{\prime}\right\} \bar{\gamma}_{0}, \quad \Gamma_{0} \equiv \gamma_{0}^{2} /\left(\gamma_{0}+1\right) \\
& E=\gamma_{0}\left\{\left(c P^{\prime} \cdot \bar{v}_{0}\right)+E^{\prime}\right\}
\end{aligned}
$$

Since $c P^{\prime}=c \psi^{\prime}, C P^{\prime}=c P^{\prime} \psi^{\prime}$, the di:ection transformation (9.2) may be derived more simply from the first three equations of (2). In fact, we find

$$
\begin{aligned}
& a_{x}=\gamma_{0}\left(a_{x}^{\prime}+p^{\prime}\right) /\left(c p / c p^{\prime}\right) \\
& a_{y}=a_{y}^{\prime} /\left(c p / c p^{\prime}\right) \\
& a_{z}=a_{z}^{\prime} /\left(c p / c p^{\prime}\right)
\end{aligned}
$$

where

$$
\rho^{\prime}=\beta_{0} E^{\prime} / C p^{\prime}=\beta_{0} / \beta^{\prime}
$$

Moreover $c p / c p^{\prime}$ may be evaluated from (2), since

$$
\begin{aligned}
& c p^{2}=c p_{x}^{2}+c p_{y}^{2}+c p_{z}^{2}=\gamma_{0}^{2}\left(c p_{x}^{\prime}+\beta_{o} E^{\prime}\right)^{2}+c p_{y}^{\prime 2}+c p_{z}^{\prime 2} \\
& =\gamma_{0}^{2} c p^{\prime 2}\left(a_{x}^{\prime}+p^{\prime}\right)^{2}+c p^{\prime 2}\left(1-a_{x}^{\prime 2}\right) \text {. Hence we have } \\
& c p / c p^{\prime}=\gamma_{0} D^{\prime}, \quad D^{\prime}=\left\{\left(a_{x}^{\prime}+p^{\prime}\right)^{2}+\gamma_{0}^{-2}\left(1-a_{x}^{\prime 2}\right)\right\}^{\frac{1}{2}}
\end{aligned}
$$

Comparison with (9.2) shows that necessarily

$$
c p / c p^{\prime}=\gamma_{0}\left(d^{\prime} B / B^{\prime}\right)
$$


a relation which is clear on the simpler grounds

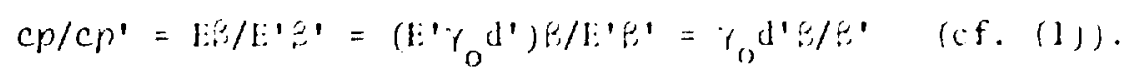

The special case $e=0, P^{\prime}=1$ of $(7-9)$ has been given in $(9.0)$.

These results, and other consequences of the (cPli) transformation are collected in 313 , where their significance becomes more apparent in a geometric sctting.

Note 1. If $\left(\mathrm{CP}_{i}, \mathrm{l}_{\mathrm{i}}\right)$ ' $\left(\mathrm{CP}_{\mathrm{i}}^{\prime}, \mathrm{l}_{\mathrm{i}}^{i}\right), \mathrm{i}=1, \ldots .$. , I under the (clli)

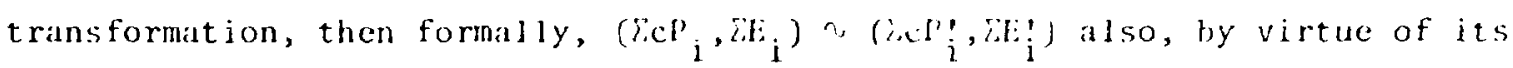
linearity. Moreover, we know $\left.(\mathrm{cP})^{2} \cdot \mathrm{E}^{2}=(\mathrm{cl})^{\prime}\right)^{2}-\mathrm{E}^{\prime 2}$ for any such corresponding pair. It follows that not only are all the $\left.(c)_{i}\right)^{2}-i_{i}^{2}$ invariant but $\left(\sum c P_{i}\right)^{2}-\left(\sum_{i}\right)^{2}$ as well. In particular, when $1=2$, we have the iden.. tity $\left(c P_{1}+c P_{2}\right)^{2}-\left(E_{1}+E_{2}\right)^{2} \equiv\left(c P_{1}\right)^{2}-E_{1}^{2}+\left(c P_{2}\right)^{2}-1:_{2}^{2}+2\left(c P_{1} \cdot c P_{2}-E_{1} E_{2}\right)$, and conclude that $\mathrm{CP}_{1} \cdot \mathrm{CP}_{2}-\mathrm{E}_{1} \mathrm{E}_{2}$ is an invariant also. For example, to obtain the energy $E_{1}^{\prime}$ of a particle 1 , in the rest frame $\Sigma$ ' of a second material particle 2 , these having known parameters $c P_{i}, E_{i}$ in $\Sigma$, one need only note that, since $c P_{2}^{\prime}=0$ and $E_{2}^{\prime}=e_{2}$ in $\Sigma^{\prime}$, one must have

$$
\begin{aligned}
& { }^{c P_{1}} c P_{2}-E_{1} E_{2}=0-E_{1}^{\prime} e_{2} \text { so that } \\
& E_{1}^{\prime}=\left(E_{1} E_{2}-c P_{1} c P_{2}\right) / e_{2} .
\end{aligned}
$$

The whole story may of course be obtained from the inverse (cPE) transformation, with the parameters

$$
\gamma_{0}=E_{2} / e_{2} \quad \beta_{0}=c p_{2} / E_{2} \quad \Psi_{0}=c P_{2} / c p_{2} .
$$


Note 2. The relativistic invariants

$$
R^{2}-c^{2} t^{2} \text { and }(c P)^{2}-E^{2}
$$

involve the same parameters as the lleisenberg uncertainty principles

$$
|C R| \cdot|\Delta P| \because h / 2 \pi=\operatorname{Ct} \Delta \mathrm{E}
$$

For the significance of this, and the invariance status of the principles in relativistic quantum mechanics, I have no reference.

Note 3. The transformation (2) provides an easy method of finding the energy and direction in $Z$. From $E=\gamma_{0}\left(\beta_{0} c p_{x}^{\prime}+E^{\prime}\right)$, one can obtain $c p=\left(E^{2} \cdot c^{2}\right)^{1 / 2}$ and hence $\psi=\left(c p_{x} / c p, c p_{y} / c p, c p_{z} / c p\right)$.

11. The force transformation. Let $(R(t), t) \sim\left(R^{\prime}\left(t^{\prime}\right), t^{\prime}\right)$ be the corresponding trajectorijes of a particle in the frames $\Sigma, \Sigma^{\prime}$. On the parallel axis $A, A^{\prime}$ we have from $(10.6)$ and $(5.8)$

$$
\begin{aligned}
c P & =c P^{\prime}+\left\{\Gamma_{o}\left(c P^{\prime} \cdot \bar{V}_{o}\right)+\gamma_{o} E^{\prime}\right\} \bar{V}_{o} \\
\tau & =\gamma_{o}\left\{\left(R^{\prime} \cdot \bar{V}_{o}\right)+\tau^{\prime}\right\}
\end{aligned}
$$

By definition, we have for the force in $\Sigma^{\prime}$,

$$
F^{\prime}=d P^{\prime} / d t^{\prime}=d\left(c P^{\prime}\right) / d \tau^{\prime}
$$

while for the corresponding force in $\Sigma$,

$$
F=d(c P) / d \tau=\left(d(c P) / d \tau^{\prime}\right) /\left(d \tau / d \tau^{\prime}\right)
$$


where

$$
\mathrm{d} \tau / \mathrm{d} \tau^{\prime}=\gamma_{0}\left[\left(\bar{V} \cdot \cdot \bar{v}_{0}\right)+1\right\}=\gamma_{0} d^{\prime}
$$

Finally, we see from (1.6) applied in $\Sigma$ ' that

$$
\mathrm{dE}^{\prime} / \mathrm{d} \tau^{\prime}=c^{-1} d E^{\prime} / d t^{\prime}=c^{-1} F^{\prime} \cdot V^{\prime}=F^{\prime} \cdot \overline{V^{\prime}}
$$

Combining all these results gives the vector form of the force transformation on the $\operatorname{axes} A, A^{\prime}$,

$$
\begin{aligned}
& F=\left(\gamma_{0} d^{\prime}\right)^{-1}\left[F^{\prime}+\left[\Gamma_{0}\left(F^{\prime} \cdot \bar{V}_{0}\right)+\gamma_{0}\left(F^{\prime} \cdot \bar{V}^{\prime}\right)\right] \bar{V}_{0}\right] \\
& d^{\prime}=1+\left(\bar{V} \cdot \cdot \bar{V}_{0}\right), \quad \Gamma_{0}=\gamma_{0}^{2} /\left(\gamma_{0}+1\right)
\end{aligned}
$$

Note 1. A remarkable consequence of (6) is the transformation to $\Sigma$ of a central force in $\Sigma^{\prime}$. Suppose at the event $\left(R^{\prime}, \tau^{\prime}\right)$ in $\Sigma^{\prime}$ a particle is subject to the central force

$$
F^{\prime}=K^{\prime} \Delta R^{\prime} /\left|\Delta R^{\prime}\right|^{3} \equiv C^{\prime} \Delta R^{\prime} ; \quad K^{\prime}<0 \text { constant }
$$

where $\Delta R^{\prime} \equiv R^{\prime}-R_{0}^{\prime}$, and $R_{0}^{\prime}$ is fixed in $\Sigma^{\prime}$. If $\Sigma^{\prime}$ moves with constant velocity $v_{0}$ in $\Sigma$, the force in $\Sigma$ is seen to be

$$
F=C^{\prime} \gamma_{0}\left[d \Delta R^{\prime}+\left\{d \Gamma_{0}\left(\Delta R^{\prime} \cdot \bar{V}_{0}\right)+\gamma_{0} d\left(\bar{V}^{\prime} \cdot \Delta R^{\prime}\right)\right\} \bar{V}_{0}\right]
$$

using the relation $\left(\gamma_{0} d^{\prime}\right)\left(\gamma_{0} d\right)=1$ of (8.5). 
Our object is to write (8) entirely in terms of $\sum$ variables. To this end, we consider two corresponding events

$$
(R, \tau) \sim\left(R^{\prime}, \tau^{\prime}\right) \quad\left(R_{0}, \tau\right) \sim\left(K_{0}^{\prime}, \tau^{\prime \prime}\right)
$$

where $R_{o}^{\prime}$ is conistant, and $R, R_{c}$ are the $\Sigma$-positions of the particle and the force center observed at the same time in $\Sigma$. For these two events, we have from the inverse of $(5.8)$

$$
\begin{aligned}
& R^{\prime}=R+\left\{\Gamma_{0}\left(R \cdot \bar{V}_{0}\right)-\gamma_{0} \tau\right\} \bar{V}_{0} \\
& R_{o}^{\prime}=R_{0}+\left\{\Gamma_{0}\left(R_{0} \cdot \bar{V}_{0}\right)-\gamma_{0} \tau\right\} \bar{V}_{0}
\end{aligned}
$$

and therefore

$$
\Delta R^{\prime}=\Delta R+\left\{\Gamma_{0}\left(\Delta R \cdot \bar{V}_{0}\right)\right\} \bar{V}_{0}
$$

where $\Delta R=R-R_{0}$ at $\tau$ in $\Sigma$.

Substitution of (9) for the first $\Delta R^{\prime}$ in (8) gives

$$
F=C^{\prime} \gamma_{0}\left[d \Delta R+\left[d \Gamma_{0}\left(\left(\Delta R+\Delta R^{\prime}\right) \cdot \bar{V}_{0}\right)+\gamma_{0} d\left(\bar{V}^{\prime} \cdot \Delta R^{\prime}\right)\right\} \bar{V}_{0}\right]
$$

We reach our goal by showing that the scalar $S$ in brackets here is actual1y

$$
S=\bar{V} \cdot \Delta R
$$

From the inverse of ( 8.17$)$ we obtain 


$$
\left(\gamma_{0} d\right) \bar{V} \cdot=\bar{V}+\left\{\Gamma_{0}\left(\bar{V} \cdot \bar{V}_{0}\right)-\gamma_{0}\right\} \bar{V}_{0}
$$

Hence it follows from (9) that the last term of $S$ may be written

$$
\begin{aligned}
& \bar{V} \cdot\left(\Delta R+\Gamma_{0}\left(\Delta R \cdot \bar{V}_{0}\right) \bar{V}_{0}\right)+\left\{\Gamma_{0}\left(\bar{V} \cdot \bar{V}_{0}\right)-\gamma_{0}\right\}\left(\Delta R \cdot \cdot \bar{V}_{0}\right) \\
& =\bar{V} \cdot \Delta R+\Gamma_{0}\left(\left(\Delta R+\Delta R^{\prime}\right) \cdot \bar{V}_{0}\right)\left(\bar{V}_{0} \cdot \bar{V}_{0}\right)-\gamma_{0}\left(\Delta R^{\prime} \cdot \bar{V}_{0}\right)
\end{aligned}
$$

Thus for (11) it will suffice to prove

$$
d \Gamma_{0}\left(\left(\Delta R+\Delta R^{\prime}\right) \cdot \bar{V}_{0}\right)+\Gamma_{0}\left(\left(\Delta R+\Delta R^{\prime}\right) \cdot \bar{V}_{0}\right)\left(\bar{V} \cdot \bar{v}_{0}\right)-\gamma_{0}\left(\Delta R^{\prime} \cdot \bar{V}_{0}\right)=0
$$

But $d=1-\left(\bar{V} \cdot \bar{V}_{0}\right)$, so this is equivalent to

$$
\Gamma_{0}\left(\Delta R+\Delta R^{\prime}\right) \cdot \bar{V}_{0}=\gamma_{0}\left(\Delta R^{\prime} \cdot \bar{V}_{0}\right)
$$

or

$$
\Gamma_{0}\left(\Delta R \cdot \bar{v}_{0}\right)=\left(\gamma_{0}-\Gamma_{0}\right)\left(\Delta R^{\prime} \cdot \bar{v}_{0}\right)
$$

Since $\Gamma_{0}=\gamma_{0}^{2} /\left(\gamma_{0}+1\right)$, we have to show

$$
\gamma_{0}\left(\Delta R \cdot \bar{V}_{0}\right)=\left(\Delta R \cdot \cdot \bar{V}_{0}\right)
$$

or using (9),

$$
\begin{array}{r}
\gamma_{0}\left(\Delta R \cdot \bar{v}_{0}\right)=\Delta R \cdot \bar{v}_{0}+\Gamma_{0}\left(\Delta R \cdot \bar{v}_{0}\right) \bar{v}_{0}^{2} \\
\text { i.e., } \quad\left(\gamma_{0}-1\right)\left(\Delta R \cdot \bar{v}_{0}\right)=\frac{\gamma_{0}^{2}}{\gamma_{0}+1} \cdot\left(\Delta R \cdot \bar{v}_{0}\right) \beta_{0}^{2}
\end{array}
$$


and this is clear since $\left(\gamma_{0}^{2}-1\right)=\gamma_{0}^{2} \beta_{0}^{2}$.

Thus (11) is established, and (10) reads

$$
\begin{aligned}
F & =C^{\prime} \gamma_{0}\left[\left(1-\bar{V} \cdot \bar{V}_{0}\right) \Delta R+(\bar{V} \cdot \Delta R) \bar{V}_{0}\right] \\
& =C^{\prime} \gamma_{0}\left[\Delta R-\left(\bar{V} \cdot \bar{V}_{0}\right) \Delta R \cdot(\bar{V} \cdot \Delta R) \bar{V}_{0}\right] \\
& =C^{\prime} \gamma_{0}\left[\Delta R+\left(\bar{V}_{x}\left(\bar{V}_{0} x \Delta R\right)\right)\right]
\end{aligned}
$$

We conclude that a particle at $\left(R^{\prime}, \tau^{\prime}\right)$ in $\Sigma^{\prime}$, subject to a central force $F^{\prime}=K^{\prime} \Delta R^{\prime} /\left|\Delta R^{\prime}\right|^{3}, \Delta R^{\prime}=R^{\prime}-R_{0}^{\prime}, R_{0}^{\prime}$ fixed in $\Sigma^{\prime}$, appears in $\Sigma$ to be subject to a force

$$
\begin{aligned}
F & =\frac{K^{\prime} \gamma_{0}}{\left|\Delta R^{\prime}\right|^{3}}\left[\Delta R+\bar{V}_{X}\left(\bar{V}_{0} x \Delta R\right)\right] \\
\Delta R^{\prime} & \equiv \Delta R+\Gamma_{0}\left(\Delta R \cdot \bar{V}_{0}\right) \bar{V}_{0}
\end{aligned}
$$

$\Delta R=R-R_{0}$ being the $\Sigma$ vector from center of force to particle when both are observed simultaneously in $\Sigma$.

Although relativity does not recognize an instantaneously transmitted force, two approximate cases are of interest.

(a) If $q_{o}$ is a fixed charge at $R_{0}^{\prime}$ in $\Sigma^{\prime}$, and the particle at $\left(R^{\prime}, \tau^{\prime}\right)$ has charge $q$, then $K^{\prime}=q q_{o}$ in Coulomb's 1 aw, and the $\sum$ force would appear as

$$
F=\gamma_{0}\left[\frac{q q_{o}}{\left|\Delta R^{\prime}\right|^{3}} \Delta R+q \bar{V} \times H\right]
$$

where $\quad H=q_{0} \bar{v}_{0} \times \Delta R /\left|\Delta R^{\prime}\right|^{3}$ 
If we take $\gamma_{0} \simeq 1$ and $\Delta R^{\prime} \simeq \Delta R$, it is apparent that the first term in (13) represents the Coulomb force, while the second is the Lorentz force on $q$ at $(R, \tau)$ due to its velocity $V$ in a r.agnetic field $H$. Moreover, the equation for $H$ in (14) expresses the Biot-Savart law for the magnetic field at $(R, \tau)$ due to the charge $q_{0}$ with velocity $v_{0}$ at $\left(R_{0}, \tau\right)$.

(b) If $m_{0}$ is a mass fixed at $R_{0}^{\prime}$ in $\Sigma^{\prime}$, and the particle at $\left(R^{\prime}, \tau^{\prime}\right)$ has mass $M^{\prime}$, then $K^{\prime}=-G M^{\prime} m_{0}$ in Newton's 1aw. Moreover, transformation of mass from $\Sigma^{\prime}$ to $\Sigma$ involves

$$
M^{\prime}=M\left(\gamma_{0} d\right) \quad m_{0}=M_{0} / \gamma_{0}
$$

Hence the gravitational force in $\Sigma^{\prime}$ appears in $\Sigma$ as

$$
F=-\gamma_{0}\left(1-\bar{V} \cdot \bar{V}_{0}\right) \cdot \frac{G M_{0}}{\left|\Delta R^{2}\right|^{3}}\left[\Delta R+\bar{V}_{x} \times\left(\bar{V}_{0} x \Delta R\right)\right]
$$

in curious analogy to the result for Coulomb force. This aspect of gravity does not seem to have been explored. It may be noted that the second part of the force lies in the plane of $v_{0}$ and $R-R_{0}$, and would tend to produce rotation of $\mathrm{M}$ about $\mathrm{M}_{0}$.

12. Energy transformation and Doppler effect. We consider here the corresponding trajectories of an immaterial particle, as they appear on standard axes $S, S^{\prime}$ in the frames $\Sigma, \Sigma^{\prime}$ (Fig. 5.1). According to (10.1) the (postulated) law of transformation for the energy of such a particle is

$$
E=E^{\prime} \gamma_{0} d^{\prime}, \quad d^{\prime}=1+\beta_{0} a_{x}^{\prime}
$$


while its direction transformation $\Psi=\left(a_{x}, a_{y}, a_{z}\right) S^{\sim} \psi^{\prime}=\left(a_{x}^{\prime}, a_{y}^{\prime}, a_{z}^{\prime}\right) S^{\prime}$ is given by $(9.6)$ as

$$
\begin{aligned}
& a_{x}=\gamma_{0}\left(a_{x}^{\prime}+\beta_{0}\right) / \gamma_{0} d^{\prime} \\
& a_{y}=a_{y}^{\prime} / \gamma_{0} d^{\prime} \\
& a_{z}=a_{z}^{\prime} / \gamma_{0} d^{\prime}
\end{aligned}
$$

Assuming $\mathrm{h}$ invariant, and remembering the relations $\mathrm{E}=\mathrm{h} \nu, \lambda=\mathrm{c} / \nu$ $(m=0)$, we obtain from (1) the wave length transformation

$$
\lambda=\lambda^{\prime} / \gamma_{0}\left(1+\beta_{0} a_{x}^{\prime}\right)
$$

which expresses the "relativistic Doppler effect."

For example, a photon of energy $E^{\prime}$ and direction $\Psi^{\prime}=(0,1,0)_{S^{\prime}}$ in $\Sigma^{\prime}$ appears in $\Sigma$ with energy $E=E^{\prime} \gamma_{0}>E^{\prime}$, and direction $\Psi=\left(\beta_{0}, \gamma_{0}^{-1}, 0\right)_{S}$, its wave length being $\lambda=\lambda^{\prime} / \gamma_{0}<\lambda^{\prime}$ (Transverse Doppler effect).

On the other hand, if its direction in $\Sigma^{\prime}$ is $\Psi^{\prime}=(-1,0,0)_{S^{\prime}}$ opposite to the direction of $\Sigma^{\prime}$ in $\Sigma$, we have the situation of a receding source, of great importance in astronomy, and we find that in $\Sigma$

$$
E=E^{\prime} \gamma_{0}\left(1-\beta_{0}\right)<E^{\prime}, \quad \lambda=\lambda^{\prime} \gamma_{0}\left(1+\beta_{0}\right)>\lambda^{\prime}
$$

the direction being of course $\Psi=(-1,0,0) \mathrm{S}$.

The accompanying "red shift" is seen to be

$$
z \equiv\left(\lambda-\lambda^{\prime}\right) / \lambda^{\prime}=\gamma_{0}\left(1+\beta_{0}\right)-1=\left(\frac{1+\beta_{0}}{1-\beta_{0}}\right)^{\frac{1}{2}}-1=\beta_{0}+\frac{1}{2} \beta_{0}^{2}+\ldots
$$


If a source at $0^{\prime}$ in $\Sigma^{\prime}$ emits a ray of $N^{\prime}$ such photons per sec and hence an energy $E^{\prime}=N^{\prime} E^{\prime} \mathrm{erg} / \mathrm{sec}$, it follows from (4) and (6.7) that the rate of arrival of energy at 0 in $\Sigma$ is

$$
E=E^{\prime} \gamma_{0}^{2}\left(1-\beta_{0}\right)^{2}=E^{\prime}\left(1-\beta_{0}\right) /\left(1+\beta_{0}\right)<E^{\prime} \mathrm{erg} / \mathrm{sec}
$$

independent of the distance to the source.

Note 1. (Flux-now/distance-now relation.) Suppose a distant isotropic source of photons at $0^{\prime}$ in its own rest frame $\Sigma^{\prime}$, moving with constant velocity $v_{0}=\left(v_{0}, 0,0\right)_{S}$ in $\Sigma$, is emitting a constant number $N^{\prime}$ of photons/sec, each of energy $E^{\prime}$, its (rest) luminosity being $L^{\prime}=N^{\prime} E^{\prime}$. Such photons, emitted at time $t^{\prime}$ in $\Sigma^{\prime}$, appear in $\Sigma$ at

$$
x=\gamma_{0} \beta_{0} c t^{\prime}, \quad t=\gamma_{0} t^{\prime}
$$

and are received at 0 in $\Sigma$ at time

$$
T=t+x / c=\gamma_{0}\left(1+\beta_{0}\right) t^{\prime}
$$

(cf. (6.6)). The $\Sigma$ event of arrival $(0, T)$ corresponds to a $\Sigma$ ' event $\left(X^{\prime}, T^{\prime}\right)$ where

$$
X^{\prime}=-\gamma_{0} \beta_{0} c T
$$

The $\Sigma^{\prime}$ numerical flux at this distance from the fixed source at $O^{\prime}$ is $N^{\prime} / 4 \pi X^{2}$. Now if $A$ is the area of the detector at 0 , perpendicular to the 
motion, then the corresponding area in $\Sigma^{\prime}$ at the event of reception is $A^{\prime}=A$. The number of photons/sec leaving $0^{\circ}$ in an essentially parallel beam so as to hit $A^{\prime}$ is $\left(N^{\prime} / 4 \pi X{ }^{2}\right) A^{\prime}$, and by $(6.7)$, the number/sec received at 0 in $\Sigma$ is $\left(N^{\prime} / 4 \pi X{ }^{2}\right) A^{\prime} \cdot \gamma_{0}\left(1-\beta_{0}\right)$. Dividing by $A=A^{\prime}$ gives

$$
\left(N^{1} / 4 \pi X^{2}\right) \gamma_{0}\left(1-\beta_{0}\right) \text { photons } / \mathrm{sec} \mathrm{cm}^{2}
$$

received at 0 , each photon carrying the diminished energy $F=E^{\prime} \gamma_{0} \cdot\left(1-\beta_{0}\right)$, by (4). Hence the energy flux detected at $(0, T)$ is the product

$$
\phi=\left(L ' / 4 \pi X^{\prime}\right) \gamma_{0}^{2}\left(1-\beta_{0}\right)^{2} \mathrm{erg} / \mathrm{sec} \mathrm{cm}^{2}
$$

But at time $T$ in $\Sigma$, the source will be at a distance $s=\beta_{0} c T=-\gamma_{0}^{-1} X^{\prime}$ by (7), whence

$$
X^{\prime}=-\gamma_{0} s
$$

Thus from (8) we obtain

$$
\phi=\left(L 1 / 4 \pi s^{2}\right)\left(1-\beta_{0}\right)^{2} \mathrm{erg} / \mathrm{sec} \mathrm{cm}^{2}
$$

for the energy flux arriving at 0 when the receding source is at distances from 0 , the observed photons exhibiting a Doppler red shift

$$
z=\left(\frac{1+\beta_{0}}{1-\beta_{0}}\right)^{\frac{1}{2}}-1
$$


Solving the latter equation for $B_{0}$ shows that

$$
1-\beta_{0}=2 /(1+\zeta), \quad \zeta \equiv(1+z)^{2}
$$

Hence (9) can be written in the forma

$$
\phi=L^{\prime} /\left(\left(4 \pi s^{2}\right) \frac{1}{4}(1+\zeta)^{2}\right) \operatorname{erg} / \sec (M p c)^{2}
$$

where $s$ is in Mpc.

Defining the corresponding apparent and absolute magnitudes $m, M$ in the usual way $(4.12)$ we find that

$$
m=M+25-5 \log 2+5 \log s(1+\zeta), \quad \zeta \equiv(1+z)^{2}
$$

with $s$ in Mpc. This relates the observables $m, z$, with the absolute magnitude $M$ and the distance $s$ of the source at the time of observation.

Note 2. (Hubble's Law and the red shift) As noted in \$4, Hubble's law states that light from galaxies at distances $s \ll s=3000$ Mpc exhibits a red shift

$$
z \simeq s / S
$$

where $S=c / H$, and $H$ is Hubble's constant

$$
H=3.24 \times 10^{-18} \mathrm{sec}^{-1}=100(\mathrm{Km} / \mathrm{sec}) / \mathrm{Mpc}
$$


If due to radial recession, then for small $z$, (5) shows that

$$
z \simeq B_{0}
$$

whence

$$
\Delta / S \simeq z \simeq B_{0}=v_{0} / c
$$

and

$$
s / v_{0} \simeq S / c=1 / H \simeq 10^{10} \mathrm{Y}
$$

a time common to all "nearby" galaxies. This is roughly the basis for the "big bang" cosmology. Note the significance of units in the relation

$$
v_{0} \simeq H s, \quad H=100(\mathrm{~km} / \mathrm{sec}) / \mathrm{Mpc}, \quad(s<<s)
$$

with $s$ in Mpc. (For $s=S, v_{0}=c$, a poor reason for calling $S$ the radius of the observable universe.)

Note 3. (The function $\mathrm{m}(\mathrm{z})$ for $\operatorname{smal1} \mathrm{z}$ ) Under the assumption $s=\mathrm{Sz}$, $\mathrm{S}=3000 \mathrm{Mpc}$, the apparent magnitude formula (12) becomes

$$
m=M+40.88+5(\log \varepsilon)\{\ln z+\ln (1+\zeta)\}, \quad \zeta=(1+z)^{2} .
$$

Introducing the variable $w=\log \mathrm{kz}, \mathrm{k}=3 \times 10^{5}$ as in $\xi 4$, it is easy to verify that

$$
\begin{aligned}
& d m / d w=5\left\{1+\frac{2 z(1+z)}{1+\zeta}\right\} \geq 5, \quad d m / d w+5 \text { as } z \rightarrow 0 \\
& d^{2} m / d w^{2}=\frac{10 z(1+2 z+\zeta)}{(\log \varepsilon)(1+\zeta)^{2}} \geq 0, \quad d^{2} m / d w^{2} \rightarrow 0 \text { as } z \rightarrow 0
\end{aligned}
$$


The limits agree with the observations, but the inequalities are both reversed from those of $\$ 4$.

Under the alternative assumption $s=\mathrm{SB}_{0}$ in (13), the magnitude (12) reads

$$
m=M+40.88+5(\log \varepsilon)(\ln (\zeta-1))
$$

which has the same slope properties as (15).

13. The momentum ellipsoid. We saw in $\$ 10$ that corresponding momenta are related by the transformation

$$
c p_{x}=\gamma_{0}\left(c p_{x}^{\prime}+B_{0} E^{\prime}\right) \quad c p_{y}=c p_{y}^{\prime} \quad c p_{z}=c p_{z}^{\prime}
$$

when $c P=c P \Psi, \quad C P^{\prime}=c P^{\prime} \Psi^{\prime}$ are referred to standard axes $S, S^{\prime}$. This may be visualized geometrically in the following way. For a given $c P^{\prime}$, construct on axes $S^{\prime}$ the auxiliary point $Q^{\prime}=\left(X^{\prime}, Y^{\prime}, Z^{\prime}\right)_{S}$, as indicated in Fig. 1, with

$$
\begin{aligned}
& X^{\prime}=\gamma_{0} c p_{X}^{\prime}=\gamma_{0}\left(c p^{\prime} \cos \psi^{\prime}\right)=\left(\gamma_{0} c p^{\prime}\right) \cos \psi^{\prime}, \\
& Y^{\prime}=c p_{y^{\prime}}^{\prime}, Z^{\prime}=c p_{z}^{\prime} .
\end{aligned}
$$

Since $\left(X / N_{0}\right)^{2}+Y^{\prime 2}+Z^{\prime 2}=c p_{X}^{\prime 2}+c p_{y}^{\prime 2}+c p_{z}^{\prime 2}$, it is clear that, when $c P^{\prime}$ ranges over a sphere of fixed radius $c P^{\prime}$, the point $Q^{\prime}$ will range over the ellipsoid of revolution

$$
X^{\prime 2} /\left(\gamma_{0} c p^{\prime}\right)^{2}+Y^{\prime 2} / c p^{\prime 2}+Z^{\prime 2} / c p^{\prime 2}=1
$$


If we now set up, with parallel axes $S$, a space for $c P$, with origin $O_{1}^{\prime}=\left(-\gamma_{0} \beta_{0} E^{\prime}, 0,0\right) S^{\prime}$, the point $Q^{\prime}$, referred to the axes $S$, has the coordinates required of $\mathrm{CP}$ in $(1)$, and hence the vector $C_{1}^{\prime} Q^{\prime}$ represents the vector $c P$ corresponding to $\mathrm{cP}^{\prime}$. Note from the figure the obvious relation

$$
c p / c p^{\prime}=\sin \Psi^{\prime} / \sin \Psi
$$

In the mechanics of scattering of a particle with fixed energy parameters $\left(c P^{\prime}, E^{\prime}, e\right)$ in a frame $\Sigma^{\prime}$, the corresponding $\Sigma$ parameters may of course be obtained directly from (10.2). But it is also important to have formulas relating $c p$ and $a_{x}=\cos \psi$ with the angle $\psi^{\prime}$ in $\Sigma^{\prime}$, as exhibited in Fig. 1 . Moreover, the $\sum$ magnitude $\mathrm{ep}$ as an explicit function of $a_{x}$, and $a_{x}^{\prime}$ as a function of $a_{x}$ are sometimes required. These formulas we derive now. We first recall from $\$ 10$ the equations (9), (8), (7):

$$
c p=c p^{\prime} \gamma_{0} D^{\prime}, \quad D^{\prime}=\left\{\left(a_{x}^{\prime}+\rho^{\prime}\right)^{2}+\gamma_{0}^{-2}\left(1-a_{x}^{\prime 2}\right)\right\}^{\frac{1}{2}}
$$

where

$$
\begin{aligned}
& \rho^{\prime}=\beta_{0} E^{\prime} / C p^{\prime}=\beta_{0} / B^{\prime} \\
& a_{x}=\gamma_{0}\left(a_{x}^{\prime}+\rho^{\prime}\right) /\left(c p / c p^{\prime}\right)=\gamma_{0}\left(a_{x}^{\prime}+\rho^{\prime}\right) / \gamma_{0} D^{\prime}
\end{aligned}
$$

These relations allow computation of $c p, a_{x}$ as functions of $a_{x}^{\prime}$. Letting $R=c p / c p^{\prime}$ temporarily, (5) and (7) may be written as

$$
\begin{aligned}
R^{2} & =\gamma_{0}^{2}\left(a_{x}^{\prime}+\rho^{\prime}\right)^{2}+1-a_{x}^{t^{2}} \\
a_{x} R & =\gamma_{0}\left(a_{x}^{\prime}+\rho^{\prime}\right)
\end{aligned}
$$




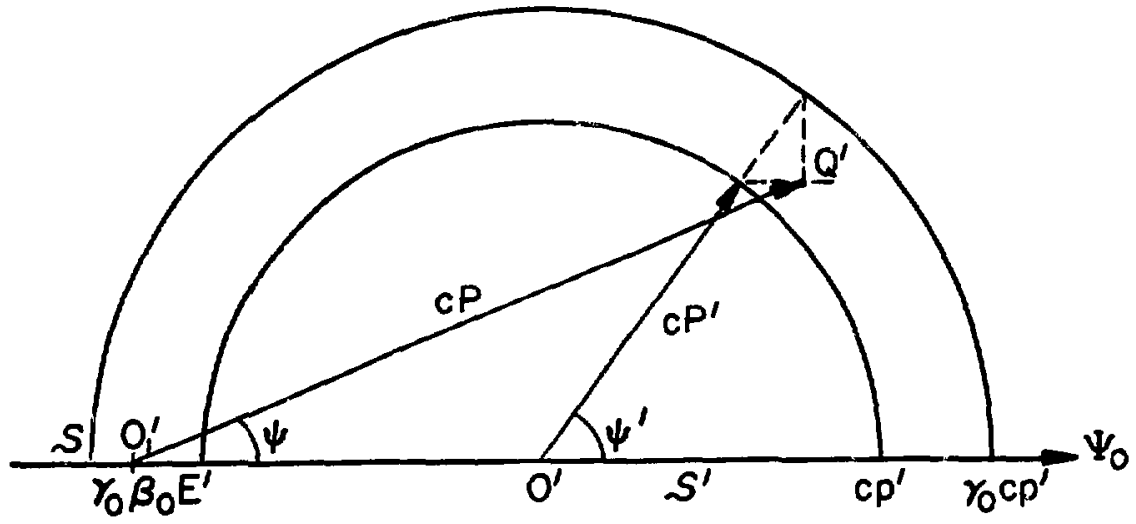

Fig. 1 .

The Momentum Ellipsoid Construction

Case I. 
From these two equations we may eliminate $a_{x}^{\prime}$ to obtain

$$
\left(\gamma_{0} / \rho^{\prime}\right)\left(1-\beta_{0}^{2} a_{x}^{2}\right) R^{2}-2 a_{x} R-\left(\gamma_{0} / \rho^{\prime}\right)\left(1-\rho^{\prime 2}\right)=0
$$

Solving for $R$ shows that $c p$ as a function of $a_{x}$ is

$$
c p=c p^{\prime} \cdot \rho \cdot\left\{a_{x}+\left(a_{x}^{2}+K\right)^{\frac{1}{2}}\right\} / \gamma_{0}\left(1-\beta_{0}^{2} a_{x}^{2}\right)
$$

where

$$
K=\gamma_{o}^{2}\left(1-\beta_{0}^{2} a_{x}^{2}\right)\left(\frac{1}{\rho^{\prime 2}}-1\right)
$$

Note here that $c p^{\prime} \cdot \rho^{\prime}=c p^{\prime} \cdot\left(\beta_{0} E^{\prime} / c p^{\prime}\right)=\beta_{0} E^{\prime}$.

It is now easy to obtain $a_{x}^{\prime}$ as a function of $a_{x}$ from the above equation $a_{x} R=\gamma_{0}\left(a_{x}^{\prime}+\rho^{\prime}\right)$ since $R$ is already known from (8). In fact

$$
a_{x}^{\prime}=\rho^{\prime}\left\{-1+\frac{a_{x}\left[a_{x} \pm\left(a_{x}^{2}+K\right)^{\frac{1}{2}}\right]}{\gamma_{0}^{2}\left(1-B_{0}^{2} a_{x}^{2}\right)}\right\}
$$

In computations where only the k.e. $k^{\prime}$ is known in $\Sigma^{\prime}$, one should recal1 the relations of $\S 1$ for a material particle:

$$
\gamma^{\prime}=1+\left(k^{\prime} / e\right), \quad \beta^{\prime}=\left(1-\gamma^{\prime}-2\right)^{\frac{1}{2}}, \quad E^{\prime}=\gamma^{\prime} e, \quad c p^{\prime}=E^{\prime} \beta^{\prime} .
$$

To obtain $k$ from $c p$ in $\Sigma$, one may use the equations $k=e(\gamma-1), \gamma=\left\{1+\left(\frac{c p}{e}\right)^{2}\right\}^{\frac{1}{2}}$. For an immaterial particle we have simply $c p^{\prime}=k^{\prime}, k=c p$.

It is clear from Fig. 1 that, as $\psi^{\prime}$ varies in any plane from $0^{\circ}$ to $180^{\circ}$, the behavior of $\psi$ will depend on the position of $O_{1}$ relative to the ellipsoid 
$E$, as indicated by the value of the fundamental ratio

$$
\rho^{\prime}=\beta_{0} / B^{\prime}=\beta_{0} E^{\prime} / C P^{\prime}=\gamma_{0} \beta_{0} E^{\prime} / \gamma_{C} c P^{\prime}
$$

There are three essentially different cases, each with its individual implications for the basic scattering formulas (5-10).

Case $I . \rho^{\prime}<1\left(K>0, \beta_{0}<\beta^{\prime}, 0_{1}^{\prime}\right.$ is inside $\left.E\right) \psi$ ranges from $0^{\circ}$ to $180^{\circ}$, with $1 \geq a_{x} \geq-1$. The $(+)$ sign is mandatory in (8) and (10), $c p$ being uniquely determined by $a_{x}$.

Under this case falls the behavior of an immaterial particle with $\beta^{\prime}=1$. Since photon scattering is important, we list the related formulas for $\rho^{\prime}=\beta_{0}$

$$
\begin{aligned}
& c p=c p^{\prime} \gamma_{0} D^{\prime}, \quad D^{\prime}=1+\beta_{0} a_{x}^{\prime}=d^{\prime} \\
& a_{x}=\gamma_{0}\left(a_{x}^{\prime}+\beta_{0}\right) / \gamma_{0} D^{\prime} \\
& c p=\gamma_{0}^{-1} c p^{\prime} /\left(1-B_{0} a_{x}\right) \\
& a_{x}^{\prime}=\left(a_{x}-\beta_{0}\right) /\left(1-\beta_{0} a_{x}\right)
\end{aligned}
$$

Case II. $\rho^{\prime}=1\left(K=0, \beta_{0}=\beta^{\prime}, 0_{1}^{\prime}\right.$ is on $\left.E\right) . \quad \psi$ ranges from $0^{\circ}$ to $90^{\circ}$ with $i \geq a x \geq 0$. The $(+)$ sign in (8) and (10) is again required, $c p$ being a single valued function of $a_{x}$. In this very special case, the scattering formulas are

$$
\begin{aligned}
& c p=c p^{\prime} \gamma_{0}^{D^{\prime}}, \quad D^{\prime}=\left\{\left(1+a_{x}^{1}\right)\left[2-\beta_{0}^{2}\left(1-a_{x}^{\prime}\right)\right]\right\}^{\frac{1}{2}} \\
& a_{x}=\left\{\left(1+a_{x}^{\prime}\right) /\left[2-\beta_{0}^{2}\left(1-a_{x}^{\prime}\right)\right]\right\}^{\frac{1}{2}}
\end{aligned}
$$




$$
\begin{aligned}
& c p=c p^{\prime}\left(2 a_{x}\right) / \gamma_{0}\left(1-\beta_{0}^{2} a_{x}^{2}\right) \\
& a_{x}^{\prime}=-1+\left[2 a_{x}^{2} / \gamma_{0}^{2}\left(1-\beta_{0}^{2} a_{x}^{2}\right)\right]
\end{aligned}
$$

Case III. $\rho^{\prime}>1\left(K<0, \beta_{0}>\beta^{\prime}, Q_{1}^{\prime}\right.$ outside $\left.E\right) . \quad \psi$ ranges from $0^{\circ}$ to a maximum $\hat{\psi}$ (opening of the tangent cone), and then back to $0^{\circ}$. Thus $1 \geq a_{x} \geq \hat{a}_{x}=\cos \hat{\psi}$, and both signs are required in (8) and (10), cp being a double valued function of $a_{x}$. Each angle $\psi<\hat{\psi}$ arrives from two distinct values of $\psi^{\prime}$, and therefore appears with two different values of $c p$. The limiting tangential value $\hat{\psi}$ is attained when $a_{x}^{2}+K=0$ in (8), and hence

$$
\hat{a}_{x}=\cos \hat{\psi}=\left\{\left(\rho^{\prime 2}-1\right) /\left(\rho^{\prime}-\beta_{0}^{2}\right)\right\}^{\frac{1}{2}}=1 /\left\{1+1 / \gamma_{0}^{2}\left(\rho^{\prime}-1\right)\right\}^{\frac{1}{2}}
$$

Using the first of these values, it appears that

$$
\gamma_{0}^{2}\left(1-\beta_{0}^{2 \wedge} a^{2}\right)=\rho^{\prime 2} /\left(\rho^{\prime 2}-\beta_{0}^{2}\right)
$$

so from (8), (13), (14) we have the corresponding value

$$
\hat{c p}=\frac{c p^{\prime} \gamma_{0}}{\rho^{\prime}}\left\{\left(\rho^{\prime}-1\right)\left(\rho^{\prime 2}-\beta_{0}^{2}\right)\right\}^{\frac{1}{2}}
$$

Similarly from (10), (13), (14) it follows that

$$
\hat{a}_{x}^{\prime}=\cos \hat{\psi}^{\prime}=-1 / \rho^{\prime}
$$

for the associated angle in $\Sigma^{\prime}$. Figure 2 shows a case of this kind. 


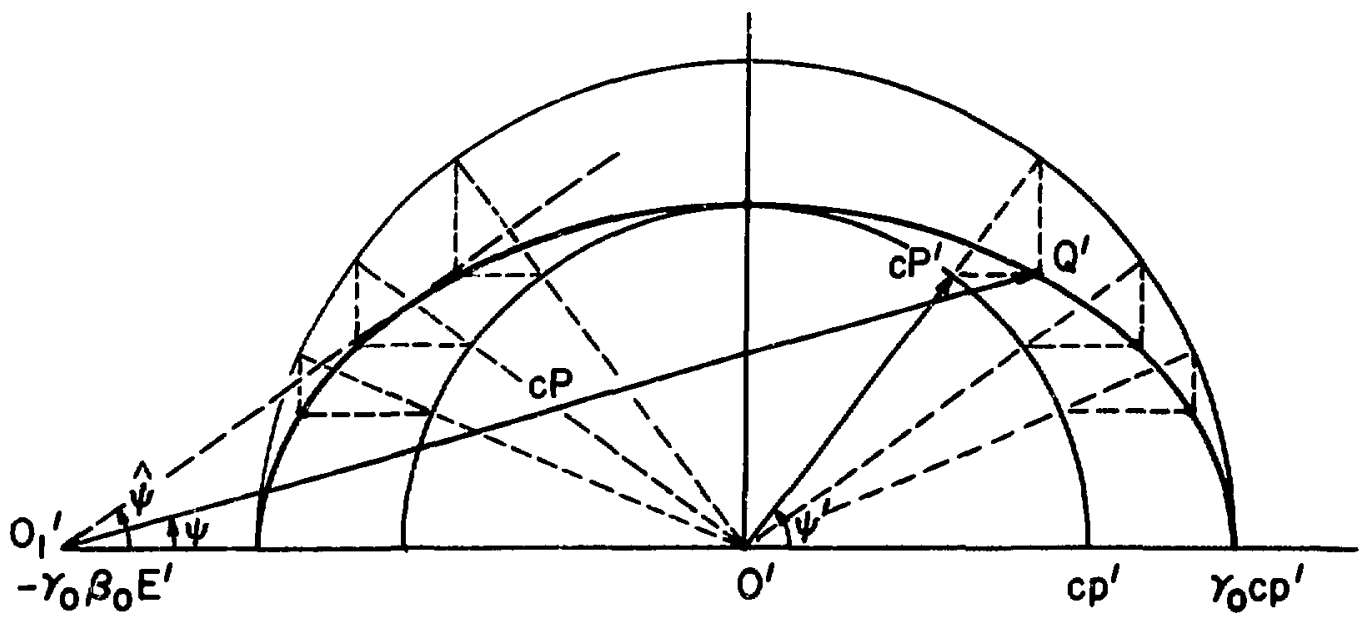

Fig. 2 .

The Momentum Ellipsoid

Case III. 
Note 1. (Geometry of the ellipse) The ellipse of Fig. 1 is of form

$$
x^{2} / a^{2}+y^{2} / b^{2}=1 \quad(b<a)
$$

where

$$
a=\gamma_{0} c p^{\prime} \quad b=c p^{\prime}
$$

and therefore has eccentricity

$$
\varepsilon=\left\{1-(b / a)^{2}\right\}^{\frac{1}{2}}=\beta_{0}<1
$$

The distance from center to focus is given by

$$
f=a \varepsilon=\gamma_{0} \beta_{0} c p^{\prime}
$$

Since $E^{\prime} B^{\prime}=c p^{\prime}$, we see that $C_{1}^{O^{\prime}}=\gamma_{0} \beta_{0} E^{\prime} \geq \gamma_{0} \beta_{0} c p^{\prime}=f$, so the point $0_{1}^{\prime}$ always lies to the left of the left focus, coinciding with it iff $B^{\prime}=1$ (immaterial particle).

In general, the ellipse (17) may be regarded as the locus of a point which maintains the fixed ratio $\varepsilon$ between its distance from the left focus and a vertical line (directrix) at distance

$$
\delta=b^{2} / a \varepsilon
$$

left of focus. With the focus as origin, the ellipse (17) has the polar form $\rho /(\delta+\rho \cos \theta) \equiv \varepsilon$

$$
\rho=\delta \varepsilon /(1-\varepsilon \cos \theta)
$$


In the momentum eliifsoid we have

$$
\delta=c p^{\prime} / \gamma_{0} \hat{p}_{0}
$$

and the polar form reads

$$
\rho=\gamma_{0}^{-1} c p^{\prime} /\left(1-\beta_{0} \cos \theta\right)
$$

For the special case of an immaterial particle just referred to, $0_{1}$ coincides with the left focus, and hence $c p$ with $\rho$ and $\psi$ with $\theta$. Thus the ellipse has the equation

$$
c p=\gamma_{0}^{-1} c p^{\prime} /\left(1-\beta_{0} \cos \psi\right)
$$

where $c p=E, c p^{\prime}=E^{\prime}$. This may be compared with the formula for $c p$ in (11c). Note 2. Since the $(R, \tau)$ and $(C P, E)$ transformations are identical, all the relations of this section may be applied to the corresponding events $(R, \tau) \sim$ $\left(R^{\prime}, \tau^{\prime}\right)$ with the obvious substitutions. In particular, for a particle undergoing the displacements $(R, \tau) \sim\left(R^{\prime}, \tau^{\prime}\right)$ with energy parameters (cP,E) $\sim\left(c P^{\prime}, E^{\prime}\right)$, the two figures are similar, since $a / b=\left(\gamma_{0} c p^{\prime}\right) / c p^{\prime}=\left(\gamma_{0} r^{\prime}\right) / r^{\prime}$, and

$$
\left|0_{1}^{\prime} 0^{\prime}\right| / b=\left(\gamma_{0} \beta_{0} E^{\prime}\right) / C p^{\prime}=\gamma_{0} \beta_{0} / \beta^{\prime}=\left(\gamma_{0} \beta_{0} \tau^{\prime}\right) / r^{\prime}
$$

Hence all directions and angles in the two figures are identical. In particular, we have from (4) the relation 
This is also clear analytically. In fact,

$$
\begin{aligned}
& c p / c p^{\prime}=E B / E^{\prime} \beta^{\prime}=\left(E^{\prime} \gamma_{0} d^{\prime}\right) B / E^{\prime} \beta^{\prime}=\gamma_{0} d^{\prime}(r / \tau) /\left(r^{\prime} / \tau^{\prime}\right) \\
& =\left(r / r^{\prime}\right) \cdot\left(\gamma_{0} d^{\prime} \tau^{\prime} / \tau\right)=r / r^{\prime} \text {, since } \\
& \tau=\gamma_{0}\left(\beta_{0} x^{\prime}+\tau^{\prime}\right)=\gamma_{0} \tau^{\prime}\left(\beta_{0}\left(x^{\prime} / \tau^{\prime}\right)+1\right)=\gamma_{0} \tau^{\prime}\left(\beta_{0} \beta^{\prime}+1\right)=\gamma_{0} \tau^{\prime} d^{\prime} .
\end{aligned}
$$

Note 3. The three cases I, II, and III may also be distinguished by the inequalities $\beta_{0}^{2} E^{\prime 2} \lesseqgtr c p^{\prime 2}=E^{\prime 2}-e^{2}$ or $\gamma_{o} e \lesseqgtr E^{\prime}$

Note 4. Equation (8) gives $c p$ as a function of $a_{x}$, double-valued in Case III $\left(\rho^{\prime}>1\right)$. Its always single valued inverse, expressing $a_{x}$ in terms of cp, can be obtained very simply from the original (cPE) transformation (10.2) thus. By eliminating $\beta_{0} c p_{x}^{\prime}$ from the equations

$$
\begin{aligned}
c p_{X} & =\gamma_{0}\left(c p_{X}^{\prime}+\beta_{0} E^{\prime}\right) \\
E & =\gamma_{0}\left(\beta_{0} c p_{x}^{\prime}+E^{\prime}\right)
\end{aligned}
$$

one obtains $E-\beta_{0} c p_{x}=\gamma_{0}^{-1} E^{\prime}$, or

$$
a_{x}=\left(E-\gamma_{0}^{-1} E^{\prime}\right) / \beta_{0} c p
$$

where $E=\left(c p^{2}+e^{2}\right)^{\frac{1}{2}}$. Indeed if one introduces the parameter $\rho^{\prime}=\beta_{0} E^{\prime} / c p^{\prime}$ in place of $E^{\prime}$ in (27) and notes that $e^{2} / c p^{\prime 2}=-1+\left(\rho^{\prime 2} / \beta_{0}^{2}\right)$ solution for $R=c p / c p^{\prime}$ leads to the quadratic from which (8) was obtained. Also, if we specialize (27) to the case $e=0, \Sigma^{\prime}=c p^{\prime}, E=c p$, we obtain again the polar equation (25). 
Example 1. A frame $\Sigma$ ' moves with speed $3 c / 5$ in $\Sigma$. A particle of rest energy 3 and k.e. 2 in $\Sigma$ ' is traveling at an angle of $60^{\circ}$ from standard axis $O X^{\prime}$. One may find its $\sum$ parameters $c p, a_{x}$ from (5-7) as follows.

$$
\begin{aligned}
& \beta_{0}=3 / 5, \quad \gamma_{0}=5 / 4 \\
& \left.k^{\prime}=2, E^{\prime}=5, c p^{\prime}=4, \rho^{\prime}=3 / 4 \text { (Case I. } \gamma_{0} c p^{\prime}=5, \gamma_{0} \beta_{0} E^{\prime}=15 / 4\right), a_{x}^{\prime}=1 / 2 \\
& D^{\prime}=\sqrt{ } 817 / 20 \\
& c p=\sqrt{ }=17 / 4 \quad a_{x}=25 / \sqrt{ } 817 . \text { The relations are shown in Fig. } 3 .
\end{aligned}
$$

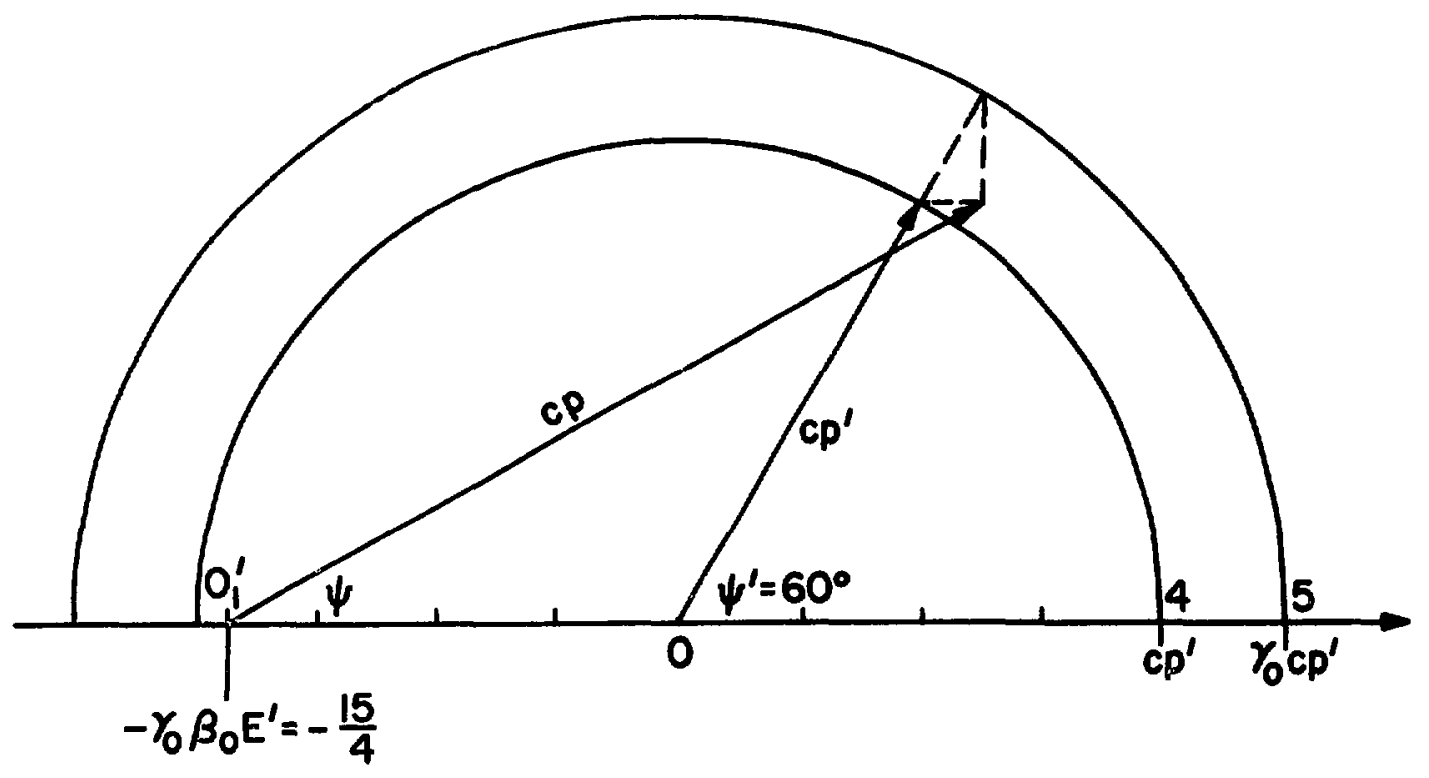

Fig. 3. An Example 
A check is provided by formulas (8-10). It may be noted that the same results may be obtained directly thus:

$$
\begin{aligned}
E & =\gamma_{0}\left(\beta_{0} c p^{\prime} \cos \psi^{\prime}+E^{\prime}\right)=31 / 4 \\
c p & =\left(E^{2}-e^{2}\right)^{\frac{1}{2}}=\sqrt{ } 817 / 4 \\
c p_{x} & =\gamma_{0}\left(c p^{\prime} \cos \psi^{\prime}+\beta_{0} E^{\prime}\right)=25 / 4 \\
a_{x} & =c p_{x} / c p=25 / \sqrt{ } 817
\end{aligned}
$$


14. Systems of particles. A system $s=S\left(e_{1}, \ldots, e_{1}\right)$ in a frame $\Sigma$ is a set of I triads $\left(C^{2}{ }_{i}, E_{i}, e_{i}\right)$ of constant, valid energy parameters $\left(E_{i}>0,\left(C P{ }_{i}\right)^{2}=E_{i}^{Z}-e_{i}^{2}\right)$ together with a set of linear trajectories $R_{i}=$ $\mathrm{R}_{i}^{0}+\bar{V}_{i} \tau$, $-\infty<\tau<\infty$, where $\bar{V}_{i}=\mathrm{cP}_{i} / \mathrm{E}_{\mathbf{i}}$, and $\tau=c t$ as usual. Thus the "mathematical object" $S$ may be considered to represent a set of I free physical particles during the time interval of its existence.

The total momentum, energy, proper energy and kinetic energy of the system are given by

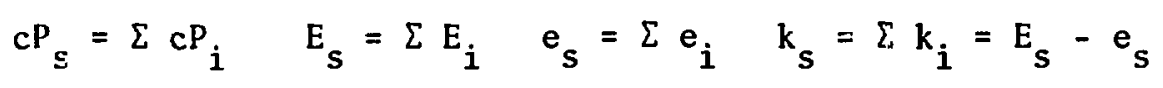

The center of mass (CM) of $S$ is defined as the point

$$
R_{s}=\sum\left(E_{i} / E_{s}\right) R_{i}
$$

and therefore

$$
\bar{v}_{s}=d_{s} / d \tau=\sum\left(E_{i} / E_{s}\right) \bar{v}_{i}
$$

indicates its velocity. Since $E_{i} \bar{V}_{i}=c P_{i}$, we infer that $\bar{V}_{s}=\sum c P_{i} / E_{s}$ and hence

$$
\mathrm{CP}_{s}=\mathrm{E}_{\mathrm{s}} \overline{\mathrm{V}}_{\mathrm{s}}
$$

A system with all velocities $V_{i}$ identical we call coherent, immaterial (with a11 $e_{i}=0$ ) if the common speed is $c$, material (with all $e_{i}>0$ ) if it is less 
It is obvious from (3) that the common velocity $v_{c}$ of a coherent system is that of its $(\mathrm{CM})$ :

$$
\bar{v}_{c}=\bar{v}_{s}
$$

Concerning systems, we prove the fundamental

Theorem 1. The totals $\mathrm{CP}_{S}, \mathrm{E}_{s}, \mathrm{e}_{s}$ of a system $\mathrm{S}$ satisfy the relations

$$
E_{s}>0 \quad\left(c P_{s}\right)^{2} \leq E_{s}^{2}-e_{s}^{2}
$$

Moreover, $\left(\mathrm{cP}_{s}\right)^{2}=E_{s}^{2}-e_{s}^{2}$ iff $S$ is coherent.

Proof. Clearly $E_{s}=\sum E_{i}>0$. The polygon and Cauchy inequalities insure that $\left|c P_{s}\right| \leq \sum\left|c P_{i}\right|=\Sigma\left(E_{i}^{2}-e_{i}^{2}\right)^{\frac{1}{2}}$

$$
\begin{aligned}
& =\Sigma\left(E_{i}-e_{i}\right)^{\frac{1}{2}}\left(\Sigma_{i}+e_{i}\right)^{\frac{1}{2}} \leq\left(\sum\left(E_{i}-e_{i}\right)\right)^{\frac{1}{2}}\left(\sum\left(E_{i}+e_{i}\right)\right)^{\frac{1}{2}} \\
& =\left(E_{s}-e_{s}\right)^{\frac{1}{2}}\left(E_{s}+e_{s}\right)^{\frac{1}{2}}=\left(E_{s}^{2}-e_{s}^{2}\right)^{\frac{1}{2}} \text {. The known }
\end{aligned}
$$

properties of the cited inequalities show that equality holds between the above extremes iff both

(a) all $\mathrm{CP}_{i}$ (equivalently ail $\overline{\mathrm{V}}_{i}$ ) are unidirectional, and

(b) $E_{i}-e_{i}=C\left(E_{i}+e_{i}\right)$ for some constant $C \geq 0$.

Clearly (b) is true iff all ratios $e_{i} / E_{i}$ are equal. But, for each $i$, $E_{i}^{2} \bar{v}_{i}^{2}=(c P)^{2}=E_{i}^{2}-e_{i}^{2}$, so (b) is also equivalent to the identity of all magnitudes $\left|\bar{V}_{i}\right|$. It follows that $\left(\mathrm{cP}_{s}\right)^{2}=E_{s}^{2}-e_{s}^{2}$ iff all vectors $\bar{v}_{i}$ are identical, i.e., $\mathrm{S}$ is coherent.

Corollary 1. For the (CM) of a system $\mathrm{S}$, one has

$$
\left|\bar{v}_{s}\right| \leq 1
$$


with equality iff $S$ is coherent immaterial.

Proof. From (4) and (6) one finds

$$
E_{s}^{2} \bar{v}_{s}^{2}=\left(\mathrm{CP}_{s}\right)^{2} \leq E_{s}^{2}-e_{s}^{2} \leq E_{s}^{2}
$$

Note 1. That $\left(\mathrm{CP}_{s}\right)^{2}=\mathrm{E}_{\mathrm{s}}^{2}-\mathrm{e}_{\mathrm{s}}^{2}$ for a coherent system may be seen directly:

$$
\begin{aligned}
E_{s}^{2} & -\left(c P_{s}\right)^{2}=E_{s}^{2}-\left(\Sigma E_{i} \bar{v}_{c}\right)^{2}=\left(\Sigma E_{i}\right)^{2}\left(1-\bar{V}_{c}^{2}\right) \\
& =\left\{\Sigma E_{i} \sqrt{ } 1-\bar{V}_{c}^{2}\right\}^{2}=\left\{\Sigma \sqrt{ } E_{i}^{2}-E_{i}^{2} \bar{v}_{c}^{2}\right\}^{2}=\left\{\Sigma \sqrt{ } E_{i}^{2}-\left(c P_{i}\right)^{2}\right\}^{2} \\
& =\left\{\Sigma e_{i}\right\}^{2}=e_{s}^{2} .
\end{aligned}
$$

15. The class of a system. Since all transmutations $A \rightarrow S$ conserve the total momentum and energy of the systems involved, it is convenient to define the class $\left\{\mathrm{CP}_{0}, \mathrm{E}_{\mathrm{o}}\right\}$ of all systems having the same totals $\mathrm{CP}_{0}, \mathrm{E}_{0}$, regardless of the number and nature of their individual proper energies $e_{i}$. A system $A$ (concurrent at an event $(R, t)$ ) being given, its totals $c_{0}, E_{0}$ define its class, and the possible systems $S$ which may result from its transmutation we shall here regard as all those in its class concurrent at $(R, t)$. The present chapter, which studies the totality of systems in a given class, is therefore of immediate relevance for Chapter III, which deals with transmutations as such, stressing the role of the initial system $A$ in determining the possible outcomes.

For the class parameters $\mathrm{CP}_{0}, \mathrm{E}_{0}$ there is first of all the validity condition of

Theorem 1. A number $E_{0}$ and vector $P_{0}$ are possible values for the total energy and momentum of some system $\mathrm{S}$, and so define a non-empty class $\left\{\mathrm{CP}_{0}, \mathrm{E}_{\mathrm{o}}\right\}$, iff 


$$
\mathrm{E}_{\mathrm{O}}>0 \text { and }\left(\mathrm{CP}_{0}\right)^{2} \leq \mathrm{E}_{0}^{2}
$$

Proof. (a) If $\mathrm{CP}_{0}, \mathrm{E}_{0}$ are the totals for some system $S$ then (l) follows from Theorem 14.1. (b) Conversely, if (1) holds, then $\mathrm{CP}_{0}, \mathrm{E}_{\mathrm{O}}$ may be regarded as totals for any system $S\left(e_{0}\right)$ of one particle, with parameters $\left(\mathrm{CP}_{0}, \mathrm{E}_{0}, e_{0}\right)$, its proper energy $e_{0} \geq 0$ being defined by the validity condition

$$
\left(\mathrm{CP}{ }_{0}\right)^{2}=E_{0}^{2}-e_{0}^{2}
$$

for a particle.

All particle parameters of $\S_{1}$ are then well-defined for such a fundamental particle $\left(C P_{0}, E_{0}, e_{0}\right)$, of class $\left\{c P_{0}, E_{0}\right\}$, and are denoted throughout by subscript 0 . In particular, its velocity, defined by $E_{0} \bar{v}_{0}=C P$ is seen from (14.4) to be the common (CM) velocity of all systems of its class.

$$
\bar{v}_{0}=\bar{v}_{s}
$$

For reasons which will become obvious, we shall call $\mathrm{v}_{\mathrm{o}}$ the class velocity and $e_{0}$ the critical energy of the class.

Corollary 1. The class velocity $v_{0}$ is the common velocity of any coherent system of class $\left\{\mathrm{CP}_{0}, \mathrm{E}_{\mathrm{o}}\right\}$, and the total momentum $\mathrm{P}_{\mathrm{O}}$ of such a system is distributed among its particles according to the relation

$$
C P_{i}=E_{i} \bar{V}_{0}=\left(E_{i} / E_{0}\right) C P_{0}
$$


Since we have defined the critical energy $e_{0}$ of the class $\left\{c P_{0}, E_{0}\right\}$ by $\left(\mathrm{CP}_{0}\right)^{2}=E_{0}^{2}-e_{0}^{2}$, the principal result of Theorem 14.1 may be stated as

Theorem 2. If $e_{s}$ is the total proper mass of a system $S$ of class $\left\{c P_{0}, E_{0}\right\}$, then necessarily

$$
e_{s} \leq e_{0}
$$

with equality iff $S$ is coherent.

Corollary 2. The coherent systems of a class $\left[\mathrm{cP}_{0}, \mathrm{E}_{0}\right\}$ have the greatest possible total proper energy $e_{s}$, and hence the least possible total kinetic energy $\mathrm{k}_{\mathrm{s}}$.

Pruof. For, $k_{s}=E_{0}-e_{s}$, where $e_{s} \leq e_{0}$ by Theoren 2,

Theorem 3. All systems $S$ of a given class are determined by the solutions, for the number $I \geq 1$, and the particle parameters $\left(\mathrm{cP}_{i}, E_{i}, e_{i}\right) i=1$, $\ldots$, I of the conditions
A. $\quad \sum \mathrm{cP}_{\mathbf{i}}=\mathrm{CP}_{\mathrm{o}}$
C. $\left(c P_{i}\right)^{2}=E_{i}^{2}-e_{i}^{2}$
B. $\Sigma \mathrm{E}_{\mathbf{i}}=\mathrm{E}_{\mathrm{o}}$
D. $E_{i}>0 \quad e_{i} \geq 0$

16. The two kinds of cli sses. We now have the picture of a class $\left\{\mathrm{cP}_{0}, \mathrm{E}_{0}\right\}$ of systems $S$, all with the same totals $\mathrm{cP}_{S}=\mathrm{CP}_{0}, \mathrm{E}_{\mathrm{S}}=\mathrm{E}_{\mathrm{O}}$. The class contains systems $S\left(e_{0}\right)$ of fundamental particles with parameters $\left(C P{ }_{0}, E_{0}, e_{0}\right) \cdot A 11$ systems of the class have the same (CM) velocity $\left(\bar{V}_{s}=\bar{V}_{0}\right)$, namely the velocity of a fundamental particle. Moreover, every system of the class has total proper energy $e_{s} \leq e_{0}$, being coherent (all velocities identical with $V_{0}$ ) iff $e_{s}=e_{0}$. 
There is a fundamental distinction between classes with $e_{0}=0$, and chose with $e_{0}>0$, containing fundamental particles which can be brought to rest by a Lorentz transformation. For the first type we have

Theorem 1. (a) A system $S\left(e_{i}\right)$ belongs to a class $\left\{\mathrm{cP}_{0}, \mathrm{E}_{0}\right\}$ with $e_{0}=0$ iff it is coherent immaterial. (b) Every such system has parameters satisfying the relations

$$
e_{i}=0 \quad E_{i} \equiv f_{i} E_{0} \quad c P_{i} \equiv f_{i} c P_{0} \quad I \geq 1
$$

where all $f_{i}>0$ and $\Sigma f_{i}=1$. (c) If $\left\{c P_{0}, E_{0}\right\}$ is a given class with $e_{0}=0$, it contains a (coherent immaterial) system $S\left(e_{i}\right)$ of any arbitrary number $I \geq 1$ of proper energies $e_{i}=0$.

Proof. (a) If $\mathrm{SE}\left\{\mathrm{CP}_{\mathrm{o}}, \mathrm{E}_{\mathrm{o}}\right\}$ with $\mathrm{e}_{\mathrm{o}}=0$, then $\mathrm{e}_{\mathrm{s}} \leq \mathrm{e}_{\mathrm{o}}$ implies $\mathrm{e}_{\mathrm{s}}=0$ (S immaterial) and $e_{S}=e_{0}(S$ coherent). Conversely, if $S$ is coherent immaterial, and $\left\{\mathrm{CP}_{0}, \mathrm{E}_{0}\right\}$ is its class, then $\mathrm{e}_{\mathrm{S}}=\mathrm{e}_{\mathrm{o}}$, since $\mathrm{S}$ is coherent, and $e_{0}=0$ since $e_{s}=0$. (b) By Corollary 15.1 , a coherent system of class $\left\{\mathrm{CP}_{0}, \mathrm{E}_{0}{ }^{\prime}\right.$ inust have $\mathrm{CP}_{i}=\left(\mathrm{E}_{\mathbf{i}} / \mathrm{E}_{0}\right) \mathrm{CP}_{0}$. Hence (b) follows if we define $f_{i}=E_{i} / E_{0}$. (c) Such 1 system may be defined by the relations

$$
\begin{array}{ll}
\mathrm{cP}_{i}=f_{i} \mathrm{cP}_{0} & \mathrm{e}_{i}=0 \\
E_{i}=f_{i} E_{0} & i=1, \ldots, I
\end{array}
$$

where the $f_{i}$ are arbitrary with $f_{i}>0, \Sigma f_{i}=1$. These parameters obviously satisfy Theorem 15.3. Note that $\left(\mathrm{CF}_{0}\right)^{2}=\mathrm{E}_{0}^{2}-0^{2}$.

Theorem 2. (a) A system $S$ is coherent material iff it belongs to a class $\left\{\mathrm{cP}_{0}, \mathrm{E}_{\mathrm{o}}\right\}$ with $\mathrm{e}_{0}>0$ and has $\mathrm{e}_{\mathrm{s}}=\mathrm{e}_{\mathrm{o}}$. (b) The parameters of such a 
systen satisfy the relations

$$
e_{i}>0 \quad E_{i}=\left(e_{i} / e_{0}\right) E_{0} \quad c P_{i}=\left(e_{i} / e_{0}\right) c P_{0} \quad I \geq 1
$$

where $\Sigma e_{i}=e_{0} \cdot$ (c) If $\left\{\mathrm{CP}_{0}{ }^{2} \mathrm{E}_{0}\right\}$ is a given class with $e_{0}>0$, and $e_{i}>0$ are any $I \geq 1$ given rest energies of sum $e_{0}$, then the class contains a (coherent diaterial) system $S\left(e_{i}\right)$ with the given I rest energies $e_{i}$.

Proof. (a) If $\operatorname{Se}\left\{\mathrm{cP} \mathrm{O}_{\mathrm{O}}, \mathrm{E}_{\mathrm{o}}\right\}$ with $\mathrm{e}_{\mathrm{O}}>0$ and has $\mathrm{e}_{\mathrm{s}}=\mathrm{e}_{\mathrm{o}}$ then $\mathrm{S}$ is coherent since $e_{S}=e_{0}$, and material since $e_{0}>0$. Conversely, if $S$ is coherent material, and $\left\{\mathrm{CP}_{0}, \mathrm{E}_{0}\right\}$ is its class, then $\mathrm{e}_{\mathrm{s}}=\mathrm{e}_{\mathrm{o}}$ since $\mathrm{S}$ is coherent, and $e_{0}>0$ since $e_{s}>0$. (b) By Corollary 15.1, a coherent system has $\mathrm{CP}_{i}=\left(\mathrm{E}_{i} / \mathrm{E}_{\mathrm{o}}\right) \mathrm{CF}_{0^{\prime}} \cdot$ Since $\mathrm{S}$ is material, with the comon velocity $V_{0}$, we have $E_{i} / E_{0}=\gamma_{0} e_{i} / \Sigma \gamma_{0} e_{i}=e_{i} / e_{0}$, and (b) follows. (c) Such a system may be defined by setting

$$
\begin{aligned}
& c P_{i}=\left(e_{i} / e_{0}\right) c P_{0} \\
& E_{i}=\left(e_{i} / e_{0}\right) E_{0}
\end{aligned}
$$

These paraneters satisfy Theorem 15.3. Note that

$$
\begin{aligned}
\left(c_{i}\right)^{2} & =\left(e_{i} / e_{0}\right)^{2}\left(c P_{0}\right)^{2}=\left(e_{i} / e_{0}\right)^{2}\left(E_{0}^{2}-e_{0}^{2}\right)=\left(\left(e_{i} / e_{0}\right) E_{0}\right)^{2}-e_{i}^{2} \\
& =E_{i}^{2}-e_{i}^{2} .
\end{aligned}
$$

Summarizing, we have identified the coherent immaterial systems as the entire contents of the classes with $e_{0}=0$, and the material ones as those particular systems of the classes with $e_{0}>0$ which have the maximai total prope energy $e_{0^{\circ}}$ Every remaining system is therefore a non-coherent member of a 
class with $e_{0}>0$, and consists of at least two particles of total proper energy $e_{s}<e_{0}$. The following sections show that every class with $e_{0}>0$ does indeed contain systems $S\left(e_{i}\right)$ of any $I \geq 2$ prescribed proper energies $e_{i} \geq 0$ of $\operatorname{sum} \sum e_{i}<e_{0}$

17. The $\Sigma^{\prime}$ frame of a class. If $\Sigma^{\prime}$ is a frame moving with any constant velocity $V_{0}\left(\left|V_{0}\right|<c\right)$ in $\Sigma$, then a free particle with (constant) parameters $\left(\mathrm{CP}_{i}, E_{i}, e_{i}\right)$ in $\Sigma$ appears in $\Sigma^{\prime}$ as a free particle with parameters $\left(\mathrm{cP}_{i}^{\prime}, E_{i}^{\prime}, e_{i}\right)$, where

$$
\left[\begin{array}{r}
\mathrm{cP}_{i} \\
E_{i}
\end{array}\right]=\mathrm{L}_{0}^{-1}\left[\begin{array}{c}
\mathrm{CP}_{i} \\
E_{i}
\end{array}\right]
$$

and $L_{0}$ is the Lorentz matrix of $\$ \$ 5,10$. Hence a system $S$ in $\Sigma$ with totals $c p_{0}, E_{0}$ appears in $\Sigma^{\prime}$ as a system $S^{\prime}$ with totals $\mathrm{cP}_{0}^{\prime}, E_{0}^{\prime}$ uniquely defined by

$$
\left[\begin{array}{r}
c P_{1}^{\prime} \\
0 \\
E_{0}^{\prime}
\end{array}\right]=L_{0}^{-1}\left[\begin{array}{c}
C P_{0} \\
E_{0}
\end{array}\right]
$$

It follows that a class of systems all with the same totals, appears as a class of systems in $\Sigma^{\prime}$ with the same totals $\mathrm{CP}_{0}^{\prime}, \mathrm{E}_{0}^{\prime}, \mathrm{i}^{\mathrm{e}} \mathrm{e}$,

$$
\left\{\mathrm{CP}_{0}, \mathrm{E}_{\mathrm{O}}\right\} \sim\left\{\mathrm{CP}, \mathrm{E}_{0}\right\}
$$

Moreover, we know from (2) and $\$ 10$ that

$$
\left(C P_{0}\right)^{2}-E_{o}^{2}=\left(C P_{0}^{\prime}\right)^{2}-E_{o}^{2}
$$

and therefore the critical energy $e_{0}$ is the same for corresponding classes: 


$$
e_{0} \sim e_{0}
$$

Hence fundamental particles are mapped into fundamental particles with corresponding energy parameters

$$
\left(C P_{0}, E_{0}, e_{0}\right) \sim\left(C P_{0}^{\prime}, E_{0}^{\prime}, e_{0}\right)
$$

Since $e_{0} \sim e_{0}, e_{s} \sim e_{s}$, and $e_{o}=e_{s}$ is the condition for coherence, we infer that coherent systems correspond in corresponding classes.

That frame $\Sigma$ ' which moves in $\Sigma$ with the class velocity $v_{0}$ of a $\Sigma$-class $\left[\mathrm{CP}_{0}, \mathrm{E}_{\mathrm{o}}\right\}$ of critical energy $\mathrm{e}_{\mathrm{o}}>0$ is called the $\Sigma$, frame of the ciass. Since $\Sigma^{\prime}$ is here the rest frame of a fundamental particle of the $\Sigma$ class, we have from (5) the corresponding parameters

$$
\left(c P_{0}, E_{0}, e_{0}\right) \sim\left(0, e_{0}, e_{0}\right)
$$

for such particles, and for their classes, the relation

$$
\left\{C P_{0}, E_{0}\right\} \sim\left\{0, e_{0}\right\}
$$

Thus a $\Sigma$ system with totals $\mathrm{CP}_{0}, \mathrm{E}_{\mathrm{O}}$ appears in $\Sigma^{\prime}$ as a system of zero total momentum, and total energy $E_{0}^{\prime}=e_{0}$, its $(C M)$ being at rest.

The parameters $\gamma_{0}, \beta_{0}$ of the Lorentz transformation from $\Sigma$ to $\Sigma$ ' are those of a fundamental particle $\left(C P_{0}, E_{0}, e_{0}\right)$, namely

$$
\gamma_{0}=E_{0} / e_{0} \quad \beta_{0}=C P_{0} / E_{0}
$$


18. Systems of zero total momentum. In an arbitrary frame $\Sigma^{\prime}$, the class $\left\{0, e_{0}\right\}\left(e_{0}>0\right)$ of systems $S^{\prime}\left(e_{i}\right)$ with total "momentum" $c P P_{0}^{\prime}=0$ and total energy $E_{0}^{\prime}=e_{0}$ has critical energy $e_{0}$ itself, and fundamental particles with energy parameters $\left(0, e_{0}, e_{0}\right)$. A11 systems of the class are determined by the conditions of Theorem 15.3, namely
A. $\quad \sum \mathrm{cP}_{i}^{\prime}=0$
C. $\left(\mathrm{CP}_{i}^{\prime}\right)^{2}=\mathrm{E}_{i}^{\prime 2}-\mathrm{e}_{i}^{2}$
B. $\sum E_{i}^{\prime}=e_{0}$
D. $\mathbf{E}_{i}>0$
$e_{i} \geq 0$
I $\geq 1$

We know that those with $e_{s}=e_{0}$ are the coherent ones, here motionless, with $\mathrm{cP}_{i} \equiv 0, E_{i}^{\prime} \equiv e_{i}$, while all others must have $e_{s}<e_{0}$ and $I \geq 2$. The simplest of these are characterized in the important

Theorem 1. (a) A system $S^{\prime}\left(e_{1}, e_{2}\right)$ of class $\left\{0, e_{0}\right\}$ with $I=2$ particles, of total proper energy $e_{s}=e_{1}+e_{2}<e_{0}$, has the unique energies

$$
\begin{aligned}
& E_{1}^{\prime}=\left(e_{0}^{2}+e_{1}^{2}-e_{2}^{2}\right) / 2 e_{0} \\
& E_{2}^{\prime}=\left(e_{0}^{2}-e_{1}^{2}+e_{2}^{2}\right) / 2 e_{0}
\end{aligned}
$$

and oppositely directed momenta of equal magnitudes, given by

$$
c p_{i}^{\prime}=\left(E_{i}^{2}-e_{i}^{2}\right)^{\frac{1}{2}}
$$

The inequality $e_{1}+e_{2}<e_{0}$ alone implies that the $E_{i}^{\prime}$ defined in (2) satisfy

$$
e_{1}<E_{1}^{\prime}<e_{0}-e_{2}, \quad e_{2}<E_{2}^{\prime}<e_{0}-e_{1} .
$$


(b) For every $e_{1} \geq 0, e_{2} \geq 0$ with $e_{1}+e_{2}<e_{0}$, there exists a system $S^{\prime}\left(e_{1}, e_{2}\right)$ of class $\left\{0, e_{0}\right\}$, the $E_{i}^{\prime}$ being defined as in (2), with the direction of $\mathrm{cP}_{1}$ arbitrary.

Proof. (a) From (1A) we have $\mathrm{CP}_{1}=-\mathrm{CP}_{2}$ so

$$
\left(\mathrm{CP}_{1}\right)^{2}=\left(\mathrm{CP}_{2}\right)^{2}
$$

Hence by (1C), $\mathrm{E}_{1}^{2}-\mathrm{e}_{1}^{2}=\mathrm{E}_{2}^{2}-\mathrm{e}_{2}^{2}$ and

$$
\mathrm{E}_{1}^{2}-\mathrm{E}_{2}^{2}=\mathrm{e}_{1}^{2}-\mathrm{e}_{2}^{2}
$$

But (1B) gives

$$
E_{1}^{\prime}+E_{2}^{\prime}=e_{0}>0
$$

and division of (3) by (4) shows that

$$
E_{1}^{\prime}-E_{2}^{\prime}=\left(e_{1}^{2}-e_{2}^{2}\right) / e_{0}
$$

Solution of the linear system $(4,5)$ then yields the values of $E_{1}^{\prime}, E_{2}^{\prime}$ in (2). From $e_{1}+e_{2}<e_{0}$ follows $e_{2}^{2}<\left(e_{0}-e_{1}\right)^{2}=e_{0}^{2}-2 e_{0} e_{1}+e_{1}^{2}$, whence $e_{1}<\left(e_{0}{ }^{2}+e_{1}{ }^{2}-e_{2}^{2}\right) / 2 e_{0} \equiv E_{1}^{\prime}$. Similarly $e_{2}<E_{2}^{\prime}$. Since $E_{1}^{\prime}+E_{2}^{\prime}=e_{0}$, we have also $E_{1}^{\prime}=e_{0}-E_{2}^{\prime}<e_{0}-e_{2}$, and $E_{2}^{\prime}=e_{0}-E_{1}^{\prime}<e_{0}-e_{1}$. (Note that $e_{1}+e_{2}=e_{0}$ implies $E_{1}^{\prime}=e_{1}, E_{2}^{\prime}=e_{2}$ for a coherent motionless system.)

(b) By the last remark, we see that the $E_{1}$, as defined by (2), are positive. Indeed,

$$
E_{i}>e_{i} \geq 0, \quad i=1,2
$$


so (1D) holds. Also the $E_{i}$ as defined have sum and difference

$$
\begin{aligned}
& E_{1}^{\prime}+E_{2}^{\prime}=e_{0} \\
& E_{1}^{\prime}-E_{2}^{\prime}=\left(e_{1}{ }^{2}-e_{2}{ }^{2}\right) / e_{0}
\end{aligned}
$$

Thus (1B) holds by (6), and multiplying (6) and (7) gives

$$
E_{1}^{2}-E_{2}^{2}=e_{1}^{2}-e_{2}^{2}
$$

or

$$
\left(E_{1}^{2}-e_{1}^{2}\right)^{\frac{1}{2}}=\left(E_{2}^{\prime 2}-e_{2}^{2}\right)^{\frac{1}{2}}
$$

Hence two oppositely directed vectors $\mathrm{cP}_{1}, \mathrm{cP}_{2}$ with the common magnitude (8) satisfy $(1 \mathrm{~A}, \mathrm{C})$, and this completes the proof.

Corollary 1. The system $S\left(e_{1}, e_{2}\right)$ of Theurem 1 has the kinetic energies

$$
\begin{aligned}
& k_{1}^{\prime}=k_{s}^{\prime}\left(e_{2}+\frac{1}{2} k_{s}^{\prime}\right) / e_{0} \\
& k_{2}^{\prime}=k_{s}^{\prime}\left(e_{1}+\frac{1}{2} k_{s}^{\prime}\right) / e_{0}
\end{aligned}
$$

$$
\text { satisfying } 0<k_{i}^{\prime}<e_{0}-e_{s}=k_{s}^{\prime}
$$

where $e_{0}$ is the total energy and $k_{s}^{r}$ the total k.e. $\left(e_{0}-e_{s}\right)$ of the system. Thus the particle of greater proper energy has the greater energy but the lesser k.e.

Proof. For example, $k_{1}^{\prime}=E_{1}^{\prime}-e_{1}=\left(e_{0}^{2}+e_{1}^{2}-e_{2}^{2}\right) / 2 e_{0}-e_{1}$

$$
\begin{aligned}
& =\left(\left(e_{0}-e_{1}\right)^{2}-e_{2}^{2}\right) / 2 e_{0}=\left(e_{0}-e_{1}-e_{2}\right)\left(e_{0}-e_{1}+e_{2}\right) / 2 e_{0} \\
& =\left(e_{0}-e_{s}\right)\left(2 e_{2}+e_{0}-e_{s}\right) / 2 e_{0}=k_{s}^{\prime}\left(e_{2}+\frac{1}{2} k_{s}^{\prime}\right) / e_{0} .
\end{aligned}
$$


For the existence theorem of the next section, we require the following generalization of Theorem $1(b)$.

Theorem 2. If $\left\{0, e_{0}\right\}$ is a given class $\left(e_{0}>0\right)$, and $e_{i} \geq 0$ are any $I \geq 2$ proper energies of sum $\sum e_{i}<e_{0}$, then the class $\left\{0, e_{0}\right\}$ contains a system $S^{\prime}\left(e_{1}, \ldots, e_{1}\right)$.

Proof. Group the given $e_{i}$ in any way (there is at least one!) into two non-empty disjoint classes $\left\{e_{j}\right\},\left\{e_{k}\right\}$, each containing only positive $e_{i}$, or only zero $e_{i}$. Define

$$
e_{j}=\Sigma e_{j} \quad e_{K}=\Sigma e_{k}
$$

where

$$
e_{J}+e_{K}=\Sigma e_{i}<e_{0}
$$

By Theorem 1, there exists a two particle system $S^{\prime}\left(e_{j}, e_{K}\right)$ of class $\left\{0, e_{0}\right\}$, its parameters satisfying the relations
A. $\quad \mathrm{cP}_{\mathrm{J}}^{\prime}+\mathrm{cP}_{\mathrm{K}}^{\prime}=0$
c. $\left(\mathrm{CP}_{\mathrm{J}}^{\prime}\right)^{2}=\mathrm{E}_{\mathrm{J}}^{2}-\mathrm{e}_{\mathrm{J}}^{2},\left(\mathrm{CP} \mathrm{K}_{\mathrm{K}}\right)^{2}=\mathrm{E}_{\mathrm{K}}^{2}-\mathrm{e}_{\mathrm{K}}^{2}$
B. $E_{j}^{\prime}+E_{K}^{\prime}=e_{0}$
D. $E_{J}^{\prime}>e_{J} \geq 0, \quad E_{K}^{\prime}>e_{K} \geq 0$

Since (A), (B) hold, it suffices to produce two systems, $S^{\prime}\left(e_{j}\right)$ of class $\left\{\mathrm{cP}_{\mathrm{J}}^{\prime}, \mathrm{E}_{\mathrm{J}}^{\prime}\right\}$, and $S^{\prime}\left(\mathrm{e}_{\mathrm{K}}\right)$ of class $\left\{\mathrm{cP}_{\mathrm{K}}^{\prime}, \mathrm{E}_{\mathrm{K}}^{\prime}\right\}$. By $(\mathrm{C})$, (D) these classes are nonempty, with critical masses $e_{J}, e_{K}$, where $\Sigma e_{j}=e_{J}, \Sigma e_{k}=e_{K}$. The desired systems must therefore be coherent, and their existence is insured by part (c) of Theorem 16.1, 16.2.

Note 1. The formulas for the $E_{i}$ in Theorem 1 (a) may be obtained more simply. For example $E_{1}^{2}-e_{1}^{2}=\left(C P_{1}^{\prime}\right)^{2}=\left(C P_{2}^{\prime}\right)^{2}=E_{2}^{\prime 2}-e_{2}^{2}=\left(e_{0}-E_{1}^{\prime}\right)^{2}-e_{2}^{2}$. 
19. The main existence theorem. The systems $S\left(e_{i}\right)$ which belong to a L-slass $\left\{c P_{0}, E_{0}\right\}$ with $e_{0}=0$ have been determined as the coherent immaterial ones of Theorem 16.1.

All systems of a class $\left\{\mathrm{CP}_{0}, \mathrm{E}_{0}\right\}$ with $\mathrm{e}_{0}>0$ must have total proper mass $e_{s} \leq e_{0}$. Those with $e_{s}=e_{0}$ are characterized as the coherent material ones of Theorem 16.2. Moreover, the correspondence of $\$ 17$ provides a oneone mapping of the systems of this $\Sigma$-class on those of the class $\left\{0, e_{0}\right\}$ in the $\Sigma$ ' frame of the class, and we have seen in Theorem 18.2 that, in addition to its coherent (motionless) systems, the latter class contains non-coherent systems $S\left(e_{i}\right)$ of any number $I \geq 2$ of arbitrarily specified proper energies $e_{i} \geq 0$ of sum $\Sigma e_{i}<e_{0}$. These remarks serve to establish the principal

Theorem 1. Given a $\Sigma$-class $\left\{C P_{0}, E_{0}\right\}$ of critical energy $e_{0}>0$, and $I \geq 1$ specified proper energies $e_{i} \geq 0$ of $\operatorname{sum} \Sigma e_{i} \equiv e_{s}$, then $\left\{c P_{0}, E_{0}\right\}$ contains no system $S\left(e_{i}\right)$ unless

$$
\mathbf{e}_{\mathbf{s}} \leq \mathrm{e}_{\mathrm{o}}
$$

Moreover, (a) if $e_{s}=e_{0}$, the class contains a system $S\left(e_{i}\right)$ iff all $e_{i}>0$, and (b) if $e_{s}<e_{0}$, such a system exists iff $I \geq 2$.

Note 1. One may now infer the following generalization of the validity condition of $\$ 1$ for particles:

$A$ number $M$ and vector $P$ are possible values for the total mass and momentun of some system of specified total proper mass $m_{s}$ iff

$M>0$ and $P^{2} \leq c^{2}\left(M^{2}-m_{s}^{2}\right)$ 
20. Systems of two particles. The results of $\$ \$ 17,18$ enable us to state

Theorem 1. All systems $S\left(e_{1}, e_{2}\right)$ of $\Sigma$-class $\left\{c P_{0}, E_{0}\right\}$ with $e_{s} \equiv e_{1}+$ $e_{2}<e_{0}$ derive, via the Lorentz transformation, from corresponding systems $S^{\prime}\left(e_{1}, e_{2}\right)$ of class $\left\{0, e_{0}\right\}$ in the $\Sigma^{\prime}$ frame of the class $\left\{\mathrm{cP}_{0}, \mathrm{E}_{0}\right\}$. The latter have the unique parameters $E_{i}, c p_{i}^{\prime}$ of $\$ 18$. The values of $E_{i}, c p_{i}$ in $\Sigma$ vary only with the direction $\Psi_{1}^{\prime}$ of $\mathrm{cP}_{1}^{\prime}$ in $\Sigma^{\prime}$.

This is exactly the kind of variation studied in $\$ 13$ (for an arbitrary velocity $V_{0}$ of $\Sigma^{\prime}$ in $\Sigma$ ); all the relations of that section apply here to either particle. Figure 1 shows the ellipsoid construction for both particles of a two-particle system simultaneously. Note the the ellipsoid is common to both, since $c P_{1}^{\prime}=c P_{2}^{\prime}$, but the origins $O_{1}^{\prime}, O_{2}^{\prime}$ of momenta $c P_{1}, c P_{2}$ are at distances $\gamma_{0} \beta_{0} E_{1}^{\prime}, \gamma_{0} \beta_{0} E_{2}^{\prime}$ from $O^{\prime}$, which are equal iff $e_{1}=e_{2}$. Each particle therefore falls under its own case $(I, I I, I I I)$, and it can be shown that all 9 combinations are possible. (Note 1.)

By reflecting the construction for the second particle in the origin $O^{\prime}$ one can visualize the situation more clearly. Thus the figure displays the necessary relation $\left|c P_{1}+c P_{2}\right|=g_{1}+g_{2}=\gamma_{0} \beta_{0} E_{1}^{\prime}+\gamma_{0} \beta_{0} E_{2}^{\prime}=\gamma_{0} \beta_{0} e_{0}=c p_{0}=$ $\left|c P_{0}\right|$, and the angle of separation $\sigma=\psi_{1}+\psi_{2}$ of the $\Sigma$ trajectories.

From the equation $c P_{0}=c P_{1}+c P_{2}$, we obtain $c p_{0}^{2}=c p_{1}^{2}+c p_{2}^{2}+2 c p_{1} c p_{2}$ $\cos \sigma=\left(c p_{1}+c p_{2}\right)^{2}-4 c p_{1} c p_{2} \sin ^{2}(\sigma / 2)$ and therefore

$$
\sin (\sigma / 2)=\left\{\left(c p_{1}+c p_{2}\right)^{2}-\left(c p_{0}\right)^{2}\right\}^{\frac{1}{2}} / 2\left(c p_{1} c p_{2}\right)^{\frac{1}{2}}
$$

Note 1.. Given $e_{0}>0$ and $0<\beta_{0}<1$, there exists a system $S^{\prime}\left(c_{1}, e_{2}\right)$ of class $\left\{0, e_{0}\right\}$ for which obtains any one of the $3 \times 3=9$ possible combinations of cases I, II, III for its two particles in the momentum ellipsoid diagram. In fact, for $i=1,2$, let $b_{i}$ be chosen on $(0,1)$ with each $b_{i}$ satisfying any 


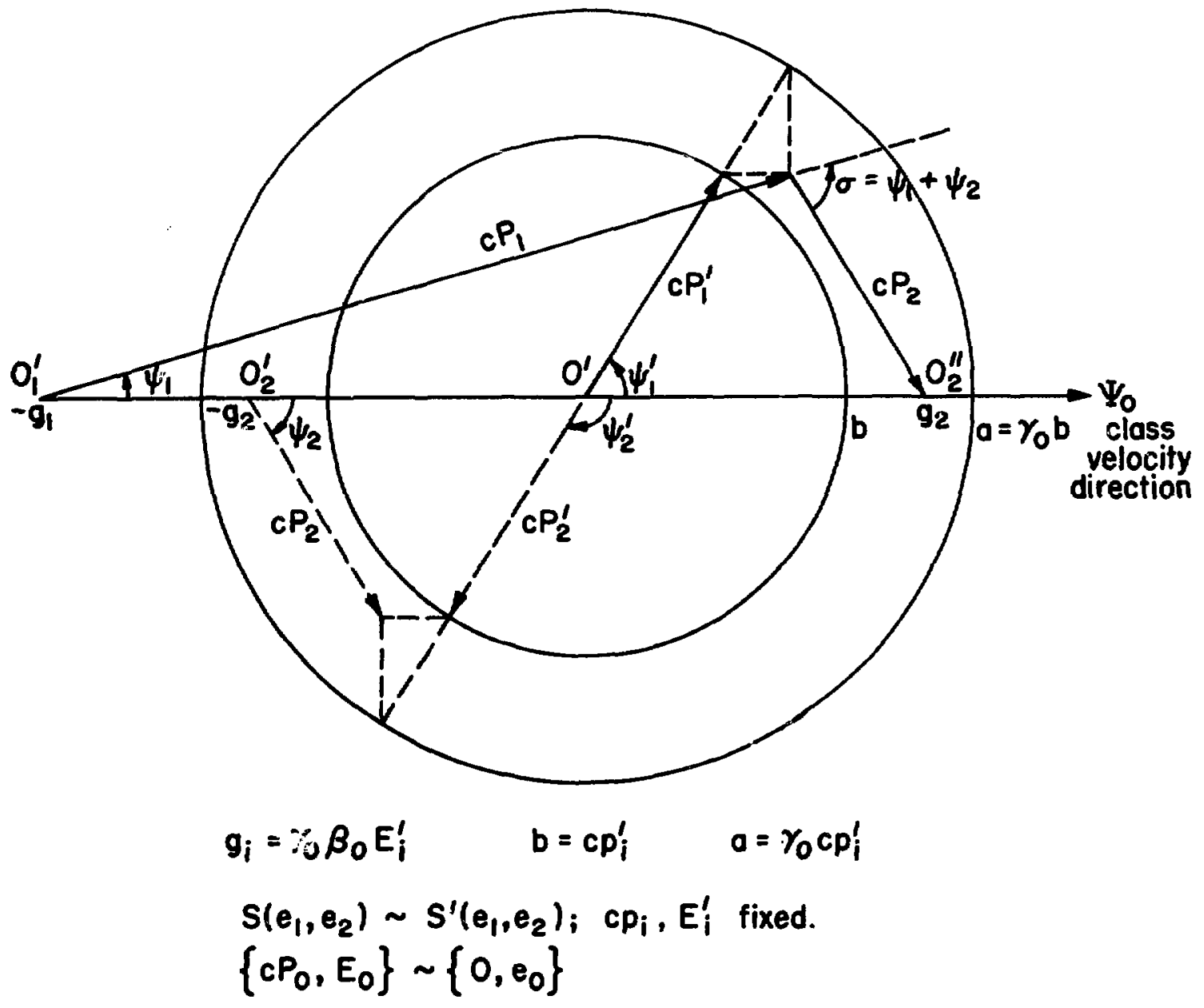

Fig. 1 .

The Two-Particle System 
one of the three inequalities $\beta_{0} \lesseqgtr b_{i}$ independently. Then define

$$
\begin{array}{ll}
E_{1}^{\prime}=b_{2} e_{0} /\left(b_{1}+b_{2}\right)>0, & E_{2}^{\prime}=b_{1} e_{0} /\left(b_{1}+b_{2}\right)>0 \\
e_{i}=E_{i}\left(1-b_{i}^{2}\right)^{\frac{1}{2}}>0, & c p_{i}^{\prime}=b_{i} E_{i}^{\prime}, \quad i=1,2 .
\end{array}
$$

One easily verifies the relations

$$
E_{i}^{\prime}+E_{2}^{\prime}=e_{0}, \quad c p_{i}^{\prime}=\left(E_{i}^{\prime 2}-e_{i}^{2}\right)^{\frac{1}{2}}, \quad c p_{i}^{\prime}=c p_{2}^{\prime}, \quad B_{i}^{\prime} \equiv c p_{i}^{\prime} / E_{i}^{\prime}=b_{i}
$$

and the above assertion follows.

Note 2. It is easy to derive the $\Sigma$ parameters of a 2-body system $S\left(e_{1}, e_{2}\right)$ of class $\left\{\mathrm{CP}_{0}, \mathrm{E}_{0}\right\}$ as functions of $\psi_{1}$ without introducing the $\Sigma$ ' frame of the class. However, their dependence on the $\Sigma^{\prime}$ angle $\psi_{1}^{\prime}$, i.s essential for understanding their behavior under cases I, II, III. For example, from $\mathrm{CP}_{1}+\mathrm{CP}_{2}=\mathrm{CP}_{0}, \mathrm{E}_{1}+\mathrm{E}_{2}=\mathrm{E}_{\mathrm{o}}$ follows at once the equations

$$
\begin{aligned}
& \left(c p_{2}\right)^{2}=\left(c p_{0}\right)^{2}+\left(c p_{1}\right)^{2}-2 c p_{0} c p_{1} \cos \psi_{1} \\
& E_{2}^{2}=E_{0}^{2}+E_{1}^{2}-2 E_{0} E_{1}
\end{aligned}
$$

Subtraction gives

$$
-e_{2}^{2}=-e_{0}^{2}-e_{1}^{2}+2\left(E_{0} E_{1}-c p_{0} c p_{1} \cos \psi_{1}\right)
$$

or

$$
E_{1}^{\prime}=\gamma_{0}\left(E_{1}-\beta_{0} c p_{1} \cos \psi_{1}\right)
$$

where we have here written $E_{1}=\left(e_{0}^{2}+e_{1}^{2}-e_{2}^{2}\right) / 2 e_{0}$ simply as an abbreviation. 
In reality it is of course the energy of 1 in $\Sigma^{\prime}$ (cf. \$18). The last equation may be put in the form $\cos \psi_{1}=\left(E_{1}-\gamma_{0}^{-1} E_{1}^{\prime}\right) / \beta_{0} c p_{1}$. One may compare this with Eq. (27) of Note 13.4.

Note 3. We consider here some properties of the $\Sigma$ separation angle $\sigma$ in the special case of equal proper energies

$$
e_{1}=e_{2}=e
$$

We have then for $i=1,2$,

$$
\begin{aligned}
& E_{i}^{\prime}=\left(e_{0} / 2\right), \quad b \equiv c p_{i}^{\prime}=\left(\left(e_{0} / 2\right)^{2}-e^{2}\right)^{\frac{1}{2}}, \quad a \equiv \gamma_{0} b=\gamma_{0} c p_{i}^{\prime} \\
& g \equiv \gamma_{0} \beta_{0} E_{i}^{\prime}=\gamma_{0} \beta_{0}\left(e_{0} / 2\right)=c p_{0} / 2 \text {, and } \rho^{\prime}=g / a . \quad \text { (Fig. 2). }
\end{aligned}
$$

The inequalities $\rho^{\prime} \leqq 1$ distinguishing the three cases are here equivalent to $e \gamma_{0} \leqq\left(e_{0} / 2\right)$.

The origins $0_{1}^{\prime}, 0_{2}^{\prime \prime}$ being symmetrically placed, it is geometrically clear from Fig. 2 and its analogues for cases II and III that the separation angle $\sigma$ has the following properties.

Case I. $\left(O_{1}^{\prime}\right.$ inside $\left.E\right) \sigma$ is least for $\psi_{1}^{\prime}=90^{\circ}$, and greatest $\left(180^{\circ}\right)$ for $\psi_{1}^{\prime}=0^{\circ}, 180^{\circ}$.

Case II. $\left(O_{1}^{\prime}\right.$ on $\left.E\right) \sigma$ is least for $\psi_{1}^{\prime}=90^{\circ}$, and greatest $\left(90^{\circ}\right)$ for $\psi_{1}^{\prime}=0^{\circ}, 180^{\circ}$.

Case III. ( $\left(O_{1}^{\prime}\right.$ outside E) $\sigma$ is greatest for $\psi_{1}^{\prime}=90^{\circ}$, and least $\left(0^{\circ}\right)$ for $\psi_{1}^{\prime}=0^{\circ}, 180^{\circ}$.

In all three cases, we have for the extremal angle $\hat{\sigma}$ occurring at $\psi_{1}^{\prime}=90^{\circ}$, 


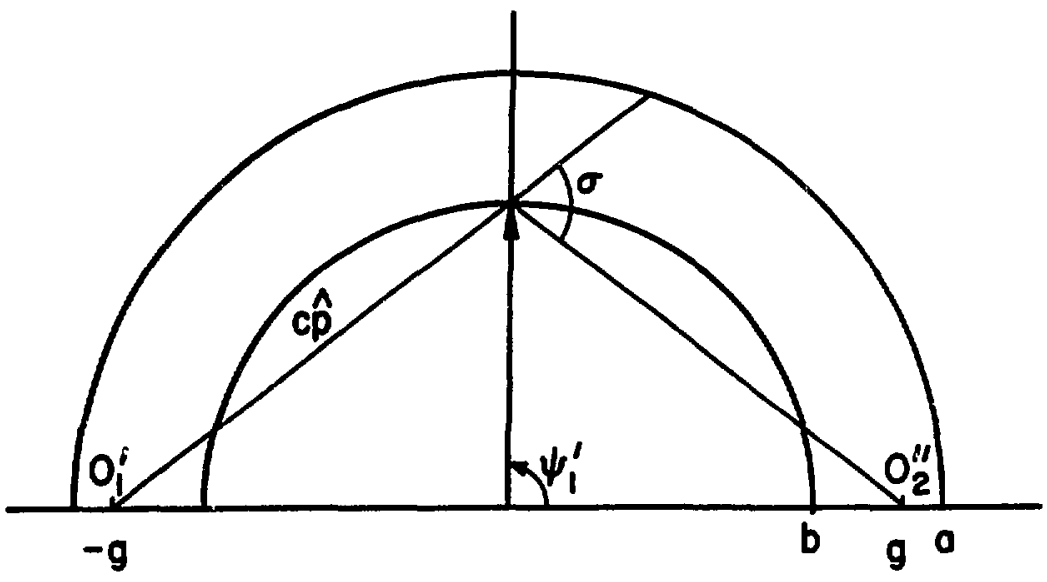

Fig. 2.

The Case $e_{1}=e_{2}$ (Case I)

$$
g=\gamma_{0} \beta_{0}\left(e_{0} / 2\right), b=c p_{i}^{\prime}, a=\gamma_{0} c p_{i}^{!}
$$




$$
\sin (\hat{\sigma} / 2)=b /\left(b^{2}+g^{2}\right)^{\frac{1}{2}}=1 /\left(1+(g / b)^{2}\right)^{\frac{1}{2}}=1 /\left(1+\left(\rho^{\prime} \gamma_{0}\right)^{2}\right)^{\frac{1}{2}}
$$

since $g / b=(g / a)(a / b)=\rho^{\prime} \gamma_{0} \cdot$

Moreover, in $\Sigma$ we see that $c p_{i}^{2}=b^{2}+g^{2}=\left(\frac{e_{0}}{2}\right)^{2}-e^{2}+\gamma_{0}^{2} \beta_{o}^{2}\left(\frac{e_{o}^{o}}{2}\right)^{2}=$ $\gamma_{0}^{2}\left(\frac{e}{2}\right)^{2}-e^{2}, i . e .$,

$$
c p_{i}=\left\{\gamma_{0}^{2}\left(-\frac{o}{2}\right)^{2}-e^{2}\right\}^{\frac{1}{2}}=\left(\left(\frac{E_{0}}{2}\right)^{2}-e^{2}\right)^{\frac{1}{2}}\left(\psi_{1}^{\prime}=90^{\circ}\right)
$$

Of geometric interest under Case $I$ is the system of two photons $\left(e \equiv 0, \rho^{\prime}=\beta_{0}<1\right)$. Since both origins $o_{1}^{\prime}, O_{2}^{\prime \prime}$ are at the foci (\$13) we see that $g=f=a \varepsilon=a \beta_{o}$, and it follows from the "string property" of the ellipse that there prevails a constant sum $c p_{1}+c p_{2} \equiv 2 a$. Hence from (1), we have for al1 $\psi^{\prime}$,

$$
\begin{aligned}
& \sin \frac{\sigma}{2}=\left(a^{2}-g^{2}\right)^{\frac{1}{2}} /\left(c p_{1} c p_{2}\right)^{\frac{1}{2}}=a\left(1-\beta_{0}^{2}\right)^{\frac{1}{2}} /\left(c p_{1} c p_{2}\right)^{\frac{1}{2}} \\
& =a \gamma_{0}^{-1} /\left(c p_{1} c p_{2}\right)^{\frac{1}{2}}=c p_{i}^{\prime} /\left(c p_{1} c p_{2}\right)^{\frac{1}{2}}=E_{i} /\left(E_{1} E_{2}\right)^{\frac{1}{2}}=\left(e_{0} / 2\right) /\left(E_{1} E_{2}\right)^{\frac{1}{2}} \\
& \geq\left(e_{0} / 2\right) /\left(\left(E_{1}+E_{2}\right) / 2\right)=e_{0} / E_{0}=1 / \gamma_{0}=\sin \frac{\sigma}{2} .
\end{aligned}
$$

The final inequality is a trivial special case of the arithmetic-geometricmean relation.

Another instance arises in the elastic scattering of a projectile on a target of equal rest mass at rest in $\Sigma$ (cf. \$30). For the $\Sigma$ ' frame of the class, the appropriate figure falls under Case II, with both $0 !$ on the ellipsoid. In such a case, $\rho^{\prime}=1$, and (2) gives for the minimum angle of separation for the scattered projectile and recoil target izrections 


$$
\sin (\hat{\sigma} / 2)=1 /\left(1+\gamma_{0}^{2}\right)^{\frac{1}{2}}
$$

or

$$
\cos (\hat{\sigma})=\left(\gamma_{0}^{2}-1\right) /\left(\gamma_{0}^{2}+1\right)
$$

Example 1. The $\Sigma$ class $\left\{\mathrm{cP}_{\mathrm{O}}=(1,2,2)_{A}, \mathrm{E}_{\mathrm{O}}=5\right\}$ has fundamental particle parameters $c p_{0}=3, \Psi_{0}=(1 / 3,2 / 3,2 / 3)_{A}, E_{0}=5, e_{0}=4, \gamma_{0}=5 / 4, \epsilon_{0}=3 / 5$. It contains no system $S\left(e_{1}, e_{2} j\right.$ with $e_{1}=2, e_{2}=3$, and none with $e_{1}=0$, $e_{2}=4$. Aside from trajectory origin, it contains exactly one such system with $e_{1}=1, e_{2}=3$. This is coherent material, with $E_{1}=5 / 4, E_{2}=15 / 4$, $\mathrm{cP}_{1}=3 / 4 \Psi_{0}, \mathrm{cP}_{2}=9 / 4 \Psi_{0}$.

The systems $s\left(e_{1}, e_{2}\right)$ with $e_{1}=0, e_{2}=2$ of the above class all derive from the corresponding systems $S^{\prime}\left(e_{1}, e_{2}\right)$ of class $\{0,4\}$ in the $\Sigma^{\prime}$ frame of the class. For $S^{\prime}$ we necessarily have $E_{1}^{\prime}=3 / 2, E_{2}^{\prime}=5 / 2$, and $c P_{i}^{\prime}=3 / 2$. In the ellipsoid figure, $\rho_{1}^{\prime}=3 / 5=\beta_{0}, \rho_{2}^{\prime}=1$ imply $o_{1}^{\prime}$ at the left focus and $O_{2}^{\prime \prime}$ on the ellipse (Cases $I$ and $\left.I I\right)$. If we specify the direction $\psi_{1}^{\prime}=(2 / 3$, $2 / 3,1 / 3)_{A^{\prime}}$ of $e_{1}$ in $\Sigma^{\prime}$, we obtain from the vectos form (10.5)

$$
\begin{aligned}
& \mathrm{cP}_{1}=(107,142,106) / 72, \quad \mathrm{CP}_{2}=(-35,2,38) / 72 \text { on } A, \\
& \mathrm{E}_{1}=23 / 8 \quad \mathrm{E}_{2}=17 / 8
\end{aligned}
$$$$
C k: \quad c P_{1}+c P_{2}=c P_{0}, \quad E_{1}+E_{2}=E_{0}, \quad E_{i}^{2}-c p_{i}^{2}=e_{i}^{2}
$$

2:. Systems of many particles. All systems $S^{\prime}\left(e_{i}\right)$ with $I \geq 2$ specified proper energies $e_{i} \geq 0$ of sum $e_{s}<e_{0}$, which belong to a class $\left\{0, e_{0}\right\}$ of an arbitrary frame $\Sigma^{\prime}$, are determined from the conditions of Theorem 15.3 , 

A. $\quad \Sigma \mathrm{cP}_{\mathbf{i}}=0$
C. $\left(\mathrm{CP}_{i}^{\prime}\right)^{2}=\mathrm{F}_{i}^{2}-\mathrm{e}_{i}^{2}$
B. $\Sigma E_{i}^{i}=e_{0}$
D. $\mathbf{E}_{\mathbf{i}}^{\mathbf{\prime}}>0$

The existence of such a system, consisting of two coherent subsystems, was proved in Theorem 18.2. Interpreting that result, with $\Sigma$ ' regarded as the $\Sigma^{\prime}$ frame of a class, we obtained a corresponding existence theorem for an arbitrary class $\left[\mathrm{CP}_{0}, \mathrm{E}_{\mathrm{o}}\right\}$ of $\Sigma$. (Theorem 19.1(b)] We now exploit the latter result to clarify the nature of those systems $S^{\prime}\left(e_{i}\right)$ of $\left\{0, e_{0}\right\}$ with $I \geq 3$ particles. These 1 ack the uniqueness properties of the two particle systems described in $\$ 18$, and we shall determine completely the energy ranges permitted for their particles. This in turn has immediate but complicated implications for an arbitrary class of the same critical energy, which will be mentioned only briefly in a later application (\$33).

Theorem 1. Let $S^{\prime}\left(e_{1}, \ldots, e_{1}\right)$ be a system of class $\left[0, e_{0}\right\}, e_{0}>0$, with $I \geq 3$ proper energies $e_{i} \geq 0$, of sum $e_{s}=\Sigma_{1}^{I} e_{i}<e_{0}$. Let $e_{1}$ be any one of the $e_{i}$, and define $e_{K}=\Sigma_{2}^{I} e_{i}$. Then there exists a two particle system $S^{\prime}\left(e_{1}, e_{K}\right)$ of class $\left\{0, e_{0}\right\}$, in which $e_{1}$ has the unique energy

$$
E_{J}^{\prime}=\left(e_{0}^{2}+e_{1}^{2}-e_{K}^{2}\right) / 2 e_{0}
$$

with

$$
e_{1}<E_{j}^{\prime}<e_{0}-e_{K}
$$

Moreover, the energy $E_{1}^{\prime}$ of $e_{1}$ in $S^{\prime}\left(e_{i}\right)$ necessarily lies on the range

$$
e_{1} \leq E_{1} \leq E_{J}^{\prime}
$$


Equality holds (a) on the left iff $e_{1}>0$ and the residual system

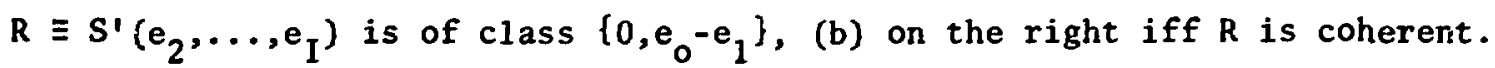

Proof. The existence of $S^{\prime}\left(e_{1}, e_{K}\right)$, and relation (1) follow from Theorem 18.1, since $e_{1}+e_{K}=e_{s}<e_{0}$. The residual system has the totals

$$
\Sigma_{2}^{I} \mathrm{cP}_{i}^{\prime}=-\mathrm{cP}_{i}^{\prime}, \quad \Sigma_{2}^{I} \mathrm{E}_{i}^{\prime}=\mathrm{e}_{0}-\mathrm{E}_{1}, \quad \Sigma_{2}^{\mathrm{I}} \mathrm{e}_{\mathrm{i}}=\mathrm{e}_{\mathrm{K}}
$$

It therefore follows from Theorem 14.1 that

$$
E_{1}^{2}-e_{1}^{2}=\left(C P_{1}\right)^{2}=\left(-C P_{1}\right)^{2} \leq\left(e_{0}-E_{1}\right)^{2}-e_{K}^{2}
$$

or equivalently,

$$
\mathrm{E}_{1} \leq\left(\mathrm{e}_{0}^{2}+\mathrm{e}_{1}^{2}-\mathrm{e}_{\mathrm{K}}^{2}\right) / 2 \mathrm{e}_{\mathrm{o}} \equiv \mathrm{E}_{\mathrm{J}}
$$

with equality iff $R$ is coherent. The statement about the lower bound of $E_{1}$ is trivial.

Corollary 1. Under the conditions of Theorem 1, the particle 1 of the system $S^{\prime}\left(e_{1}, e_{K}\right)$ has k.e.

with

$$
k_{j}^{\prime}=\frac{k_{s}^{\prime}}{e_{c}}\left(e_{K}+\frac{k_{s}^{\prime}}{2}\right)
$$

$$
0<\mathrm{k}_{\mathrm{J}}^{\prime}<\mathrm{e}_{\mathrm{o}}-\mathrm{e}_{\mathrm{s}}=\mathrm{k}_{\mathrm{s}}^{\prime}
$$

The k.e. of $e_{1}$ in $S^{\prime}\left(e_{i}\right)$ must lie on the range 


$$
0 \leq \mathrm{k}_{1}^{\prime} \leq \mathrm{k}_{\mathrm{j}}^{\prime}
$$

with the same conditions for equality.

Proof. One need only apply Corollary 18.1 in Theorem 1.

Theorem 2. Let $\left\{0, e_{0}\right\}$ be a class in $\Sigma^{\prime}$, and $e_{i} \geq 0$ any $I \geq 3$ stipulated proper energies of sum $e=\Sigma_{1}^{I} e_{i}<e_{0}$. Then, for every number $E_{i}$ and vector $\mathrm{CP}_{1}$ satisfying the validity condition

$$
E_{1}^{\prime}>0, \quad\left(C P_{1}^{\prime}\right)^{2}=E_{1}^{2}-e_{1}^{2}
$$

there exists a system $S^{\prime}\left(e_{1}, \ldots, e_{I}\right)$ in $\left\{0, e_{0}\right\}$, in which particle 1 has the parameters $\left(\mathrm{CP}_{1}, \mathrm{E}_{1}, \mathrm{e}_{1}\right)$, provided only that

$$
e_{1} \leq E_{1}<E_{J}^{\prime} \equiv\left(e_{0}^{2}+e_{1}^{2}-e_{K}^{2}\right) / 2 e_{0}
$$

If the given $E_{i}^{\prime}=E_{j}^{\prime}$, this is also true provided all $e_{2}, \ldots, e_{I}$ are of the same kind $(>0$ or $=0)$.

Proof. It suffices to produce a residual system $R=S^{\prime}\left(e_{2}, \ldots, e_{I}\right)$ with totals

$$
\Sigma_{2}^{I} c P_{i}^{\prime}=-c_{1}^{\prime}, \quad \Sigma_{2}^{I} E_{i}^{\prime}=e_{0}-E_{i}^{\prime}
$$

Since we are given in any case that $E_{i} \leq E_{j}^{\prime}<e_{0}-e_{K} \leq e_{0}$ (Theorem 18.1) we certainly know $e_{0}-E_{i}>0$. Moreover, the inequality $E_{i} \leq E_{j}^{j}$ is strictly equivalent to 


$$
\left(-c P_{1}^{\prime}\right)^{2}=\left(c P_{1}^{\prime}\right)^{2} \equiv E_{1}^{2}-e_{1}^{2} \leq\left(e_{0}-E_{1}^{\prime}\right)^{2}-e_{K}^{2}
$$

This insures that the class $\left\{-c P_{1}^{1}, e_{0}-E_{1}^{\prime}\right\}$ in which we seek the system $R$ is at least non-empty (Theorem 15.1), and moreover has a critical energy $e^{*} \geq e_{K}$, with equality iff $E_{1}^{\prime}=E_{J}^{\prime}$. But $e_{K}=\Sigma_{2}^{I} e_{i}$ is the total proper energy of the desired system $R$, and $i$ ts existence follows from Theorem 16.1 and Theorem 19.1. In detail: if $E_{1}^{\prime}<E_{J}^{\prime}$ is given, then $e^{*}>{ }{ }_{K}$, the above class has critical energy $e^{*}>0$, and contains a non-coherent system $R$ by Theorem 19.1(b) since the $e_{2}, \ldots, e_{I}$ are $I-1 \geq 2$ proper energies; if $E_{i}^{\prime}=E_{j}^{\prime}$ is given, then $e^{*}=e_{K}$, and $R$ exists as a coherent system by Theorem 16.1(c) if $e_{K}=0$, or by Theorem 19.1(a) if $e_{K}>0$, since we have stipulated in this limiting case that $e_{2}, \ldots, e_{I}$ are of the same kind.

Corollary 2. Under the conditions if Theorem 2, all values of $k_{1}$ on the range

$$
0 \leq k_{1}^{\prime} \leq k_{j}^{\prime} \equiv \frac{k_{s}^{\prime}}{e_{0}}\left(e_{K}+\frac{k_{s}^{\prime}}{2}\right) ; \quad k_{s}^{\prime}=e_{0}-e_{s}
$$

are attainable, with the same condition for the upper limit. 


\section{Chapter III \\ TRANSMUTATIONS OF SYSTEMS}

22. Transmutations. A transmutation in an event space $\Sigma$ is a localized "black box" physical process, of short duration, in which a set of free physical particles is converted into a second such set, with conservation of total eriergy and momentum. Such a process is here idealized as an event $(R, t):$

$$
A \rightarrow S
$$

at which two systems $A, S$ of the same class $\left\{\mathrm{CP}_{0}, \mathrm{E}_{\mathrm{o}}\right\}$, and concurrent at $(R, t)$, interchange "reality," A becoming "virtual" as $S$ becomes "real."

Thus the reverse process $S \rightarrow A$ does not here connote time reversal, but simply a reversed interpretation of reality for the same two mathematical objects $A$ and $S$, as indicated in Fig. 1.

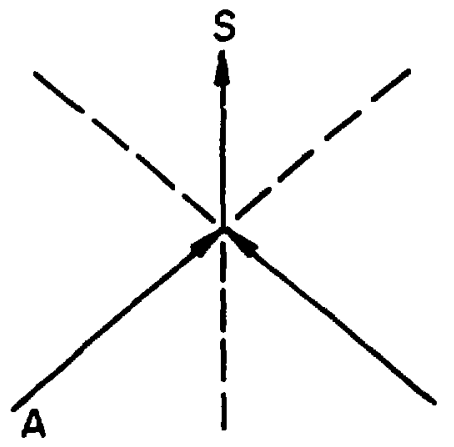

$$
A \rightarrow S
$$

(fusion)

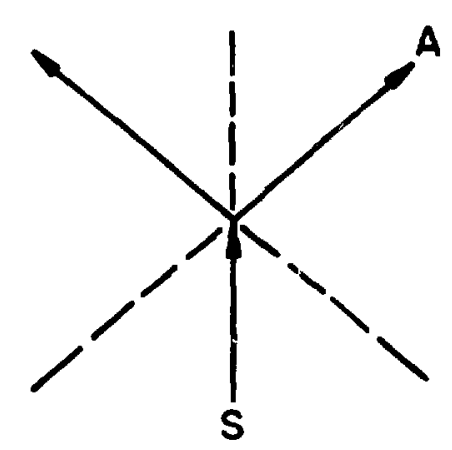

$$
\mathrm{S} \rightarrow \mathrm{A}
$$

(decoy)

Fig. 1 .

While other conservations laws (for charge, spin, ....) may impose further restrictions, we shall here regard two arbitrary systems as 
interconvertible iff they belong to the same class and are concurrent at an event.

In particular, if $S^{*}$ is a system of ciass $\left\{c P_{0}, E_{0}\right\}$, consisting of a single particle, hence with parameters $\left(\mathrm{CP}_{0}, \mathrm{E}_{0}, \mathrm{e}_{0}\right)$, and $\mathrm{A}$ and $\mathrm{S}$ are arbitrary systems of this class, concurrent with $S^{*}$ at some event $(R, t)$ of its trajectory, then the fusion $A \rightarrow S^{*}$ and the decay $S^{*} \rightarrow S$ are equally possible. Indeed, every transmutation $A \rightarrow S$ may be regarded, mathematically at least, as a composite process $A \rightarrow S^{*} \rightarrow S$, where the fundamental particle $S^{*}$ is of negligible duration.

The present chapter is, in the main, only an elaboration of Chapter II, which discussed the totality of systems belonging to a given class $\left\{\mathrm{cP}_{0}, \mathrm{E}_{0}\right\}$, that is to say, having a given total momentum $P_{0}$ and energy $E_{0} \cdot$ Here we emphasize the dependence of $\mathrm{P}_{0}$ and $E_{0}$ on the particle parameters of the initial system $A\left(e_{h}\right)$.

We state below without proof, the principal implications of Chapter II for transmutations.

Theorem 1. In any transmutation $A \rightarrow S, S$ is coherent immaterial iff A is. For such a system A, all possibie resulting systems are "coalesced," with the single trajectory of $A$, and parameters determined to the extent indicated in Theorem 16.1 .

For example, a photon of energy $E=h \nu$ can only transmute into a coherent system of immaterial particles of total energy $E$, all superimposed on its own line of flight. It cannot produce an electron-positron pair,nor a divergent set of photons.

Theorem 2. If $A \rightarrow S$ is a transmutation between systems of class $\left\{\mathrm{cP}_{0}, \mathrm{E}_{\mathrm{O}}\right\}$, both $A$ and $S$ have the same (CM) velocity, namely the class "velocity" 
$\left(\bar{V}_{0}=c P=P_{0} / E_{0}\right)$, and identical (CM) trajectories. If $\left|\bar{V}_{0}\right|<1\left(e_{0}>0\right)$, then $A \rightarrow S$ appears, in the $\Sigma^{\prime}$-frame of the class, as a transmutation $A^{\prime} \rightarrow S^{\prime}$ between corresponding systems of class $\left\{0, e_{0}\right\}$, occurring at their stationary (CM). Conservation of energy in $\Sigma$ is expressed by

$$
e_{a}+k_{a}=E_{0}=e_{s}+k_{s}
$$

and in $\Sigma^{\prime}$ by

$$
e_{a}+k_{a}^{\prime}=e_{0}=e_{s}+k_{s}^{\prime}
$$

Theorem 3. Let $A$ be a system of class $\left\{\mathrm{cP}_{0}, \mathrm{E}_{0}\right\}$ with $e_{0}>0$, and let $e_{i} \geq 0$ be any $I \geq 1$ specified proper energies. Then a transmutation $A \rightarrow S$ $\left(e_{1}, \ldots, e_{I}\right)$ is impossible unless

$$
\Sigma_{1}^{I} e_{i} \equiv e_{s} \leq e_{0}
$$

(a) If $e_{s}=e_{0}, A+S$ is possible iff all $e_{i}>0$.

(b) If $e_{S}<e_{o}, A+S$ is possible iff $I \geq 2$.

In case (a), $S$ is a completely unique coherent material system, coalesced, with the single trajectory of the (CM) of $A$, and the parameters given in Theorem 16.2. Fusion, with $I=1$, is the case of physical interest. In case (b), details on the nature of $S$ will be found in 5 \$18-21.

Note 1. We give below an outline of a general method for following a cransmutation 
$A\left(e_{h}\right)+S\left(e_{i}\right)$.

(a) From the particle parameters $e_{h}, \bar{E}_{h}$, and $\left(\mathrm{CF}_{h} j_{A}\right.$ on given $\bar{L}$ axes $A$ one obtains the total-parameters

$$
e_{a}=\Sigma e_{h}, \quad E_{0}=\Sigma E_{h}, \quad\left(c P_{0}\right)_{A}=\Sigma\left(c P_{h}\right)_{A}, \quad c p_{0}=\left|c P_{0}\right|, \quad\left(\Psi_{0}\right)_{A}=\left(c P_{0}\right)_{A} / c p_{0}
$$

The class of $A\left(e_{h}\right)$ therefore has the parameters $\mathrm{CP}_{0}, \mathrm{E}_{0}$, and $e_{0}=\left(E_{0}^{2}-c p_{0}^{2}\right)^{\frac{1}{2}} \geq e_{a}, \gamma_{0}=E_{0} / e_{0}, \beta_{0}=c p_{0} / E_{0}, \Psi_{0}$

(b) The necessary condition $e_{0} \geq \sum e_{i} \equiv e_{s}$ for formation of a proposed system $S\left(e_{i}\right)$ may be tested, although a zero cross section for the reaction makes it unnecessary. Assuming the transformation to $S\left(e_{i}\right)$ possible, in accordance with Theorem 3 , its actual occurrence, rather than that of competing reactions, depends on relative values of cross sections and chance.

(c) If indeed a non-coherent system $S\left(e_{i}\right)$ results, with $e_{0}>e_{S}$, I $\geq 2$ (the only non-trivial case), we require the $\Sigma$-parameters of its particles. It may be necessary to consider for this purpose the corresponding transmutation $A^{\prime} \rightarrow S^{\prime}$ as it appears in a frame $\Sigma^{\prime}$ moving with "velocity" $\bar{V}_{0}=\beta_{0} \psi_{0}^{\prime}$ in $\Sigma$, usually the $\Sigma^{\prime}$ frame of the class, or the rest frame of some particle of $A\left(e_{h}\right)$, and related to $\Sigma$ by the (CPE) transformation. In such cases one requires the $(\mathrm{CPE})^{-1}$ transformation to obtain $A^{\prime}$ from $A$, and (CPE) to get back from $S$ ' to $S$. These transformations involve the parameters $\gamma_{0}, \beta_{0}, \Psi_{0}$ associated with the velocity of $\Sigma^{\prime}$ in $\Sigma$. If $\Sigma^{\prime}$ is the $\Sigma$ ' frame of the class, then

$$
\gamma_{0}=E_{0} / e_{0}, \quad \beta_{0}=c p_{0} / E_{0}, \quad \Psi_{0}=\left(C P_{0}\right)_{A} / c p_{0}
$$


If $\Sigma^{\prime}$ is the rest frame of one of the (material) particles $h_{0}$ of $A$, then one uses for the (CPE) transformation the parameters

$$
\gamma_{0}=E_{h_{0}} / e_{h_{0}}, \quad \beta_{0}=c p_{h_{0}} / E_{h_{0}}, \quad\left(\Psi_{0}\right)_{A}=\left(c P_{h_{0}}\right)_{A} /\left|c p_{h_{0}}\right| .
$$

In any case, one has two alternatives for the (cPE) transformation.

(I) One may use the vector form (10.5):

$$
\begin{aligned}
& \left(c P_{h^{\prime} A^{\prime}}=\left(c P_{h}\right)_{A}+\left\{\delta_{0}\left(c P_{h} \cdot \Psi_{0}\right)-\gamma_{0} B_{0} E_{h}\right\}\left(\Psi_{0}\right) A\right. \\
& E_{h}^{\prime}=\gamma_{0}\left\{-\beta_{0}\left(c P_{h} \cdot \Psi_{0}\right)+E_{h}\right\}
\end{aligned}
$$

to obtain $A^{\prime}$ from $A$, and

$$
\begin{aligned}
& \left(\mathrm{CP}_{i}\right)_{A}=\left(\mathrm{CP}_{i}\right)_{A^{\prime}}+\left\{\hat{o}_{0}\left(\mathrm{cP} ! \cdot \Psi_{0}\right)+\gamma_{0} \beta_{0} E_{i}\right\}\left(\Psi_{0}\right)_{A} \\
& E_{i}=\gamma_{0}\left\{\beta_{0}\left(\mathrm{CP}_{i} \cdot \Psi_{0}\right)+E_{i}\right\}
\end{aligned}
$$

to get S from S'.

(II) The rotation $\delta$ defined on axes $A$ by the matrix

$$
D=\left[\begin{array}{lll}
a_{x} & -a y & -a_{z} \\
a_{y} & 1-\left(a_{y}^{2} / \Delta\right) & -a_{y} a_{z} / \Delta \\
a_{z} & -a y z z & 1-\left(a_{z}^{2} / \Delta\right)
\end{array}\right]
$$

where $\Delta=1+a_{x} \neq 0$ and $\Psi_{0}=\left(a_{x}, a_{y}, a_{z}\right)_{A}$ 
(Appendix I, Corollary 1) takes axes $A$ into a set of standard axes $S$. One can therefore obtain $\left(\mathrm{CP}_{h}\right)_{S}=\mathrm{D}^{\tau}\left(\mathrm{CP}_{h}\right)_{A}$ as the coordinates $\left(\overline{C P}_{h x}\right.$, $\left.\overline{C P}_{h y}, \overline{C P}_{h z}\right)$ of $\mathrm{CP}_{h}$ referred to $S$. The inverse of (10.2):

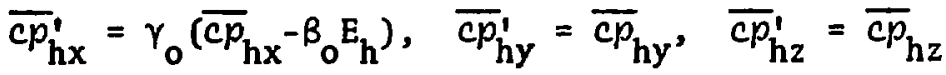

$$
\begin{aligned}
& E_{h}^{\prime}=\gamma_{0}\left(-\beta_{0}{\overline{c p_{h x}}}_{h}+E_{h}\right)
\end{aligned}
$$

then gives the system $A^{\prime}$ on standard $\Sigma^{\prime}$ axes $S^{\prime}$. From the new system $S^{\prime}$ on axes $S^{\prime}$ one next obtains the $\Sigma$ system $S$ on $S$ by means of (10.2),

$$
\begin{aligned}
& \overline{c p}_{i x}=\gamma_{0}\left(\overline{c p}_{i x}^{\prime}+\beta_{0} E_{i}^{\prime}\right), \overline{c p}_{i y}=\overline{c p}_{i y}^{\prime}, \overline{c p}_{i z}=\overline{c p}_{i z}^{\prime} \\
& E_{i}=\gamma_{0}\left(\beta_{0} \overline{c p_{i x}^{\prime}}+E_{i}^{\prime}\right)
\end{aligned}
$$

Finally, we have, relative to the original $\Sigma$ axis $A$,

$$
\left(\mathrm{CP}_{i}\right)_{A}=\mathrm{D}\left(\mathrm{CP}_{i}\right)_{S}
$$

where $D$ is defined above, and $\left(\mathrm{CP}_{i}\right)_{S}=\left(\overline{C p}_{i x}, \overline{C P}_{i y}, \overline{C P}_{i z}\right)$.

In either method, one may require the further $\Sigma$ parameters

$$
k_{i}=E_{i}-e_{i}, \quad B_{i}=c p_{i} / E_{i}, \quad\left(\Psi_{i}\right)_{A}=\left(c P_{i}\right)_{A} /\left|c P_{i}\right|
$$

and the trajectory

$$
\left(R_{i}\right)_{A}=\left(R_{i}^{0}\right)_{A}+\left(t-t_{0}\right) B_{i} c\left(\Psi_{i}\right)_{A} .
$$


In case the transmutation is studied in $\Sigma$ itself, as for example in fusion and Compton scattering on target at rest, the (cPE) transformation is of course by-passed. Fusion $(\$ 28)$, a coherent result, is trivial, since the final system $S\left(e_{0}\right)$ has the parameters $\left(\mathrm{CP}_{0}, \mathrm{E}_{0}, \mathrm{e}_{0}\right)$ of the class as obtained in (a).

(d) We have not mentioned how the resulting system is arrived at. If $e_{0}>e_{s}$, and $I \geq 2$, a non-coherent result is possible. Even in the simplest cases, it is not completely determined, and the energy, and direction referred to the axes exployed, of any particle $i$ to be followed, must be obtained by sampling physically given distributions. In cases of "non-polarized" emission about a stipulated basic direction, the direction of emission may be obtained (for Monte Carlo purposes) by the "standard device" of Appendix II.

In the case of elastic scattering on target at rest, treated in the $\Sigma^{\prime}$-frame of the class $(\xi 30)$, we have $E_{3}^{\prime}=E_{1}^{\prime}, E_{4}^{\prime}=E_{2}^{\prime}$, and only the direction $\Psi_{3}^{\prime}$ in $\Sigma '$ is required.

The above methods are illustrated in following sections.

Example 1. A photon $(h=1)$ of energy 3 and direction $(2 / 3,1 / 3,2 / 3)_{A}$ strikes a particle $(h=2)$ of rest energy $\sqrt{2}$, k.e. $2-\sqrt{2}$ and direction $(-1 / \sqrt{2}, 1 / \sqrt{2}, 0)_{A}$. The collision results in two particles $(i=3,4)$ of rest energies 1 and 2 respectively. The transmutation is treated in the $\Sigma^{\prime}$ frame of the class, and the auxiliary direction $\Omega^{\prime}=(-2 / 3,1 / 3,2 / 3)$ is chosen for location of $\Psi_{3}^{\prime}$ about the basic photon direction $\Psi_{1}^{\prime}$ in $\Sigma^{\prime}$, as explained in Appendix II. The parameters of $S\left(e_{3}, e_{4}\right)$ are required on the original axis $A$.

For $A\left(e_{1}, e_{2}\right)$ and the Lorentz parameters we have

$$
\begin{array}{llll}
\mathrm{e}_{1}=0 & \mathrm{E}_{1}=3 & \mathrm{CP} P_{1}=3 & \mathrm{CP} P_{1}=(2,1,2)_{A} \\
\mathrm{e}_{2}=\sqrt{2} & \mathrm{E}_{2}=2 & C P_{2}=\sqrt{2} & \mathrm{CP} P_{2}=(-1,1,0)_{A}
\end{array}
$$




$$
\begin{array}{llll}
e_{a}=\sqrt{ } 2 & E_{0}=5 & c P_{0}=3 & c P_{0}=(1,2,2)_{A} \\
e_{0}=4, & \gamma_{0}=5 / 4, & B_{0}=3 / 5, & \Psi_{0}=(1 / 3,2 / 3,2 / 3)_{A}
\end{array}
$$

For the proposed $S\left(e_{3}, e_{4}\right)$, we have $e_{0}>e_{s}=3, I=2$, which is possible.

Using Method (I) of Note 1, we pass to the frame $\Sigma$ ' by

(I)

$$
\begin{aligned}
& \left(\mathrm{CP}_{1}^{\prime}\right)_{A^{\prime}}=\left(\mathrm{CP}_{1}\right)_{A}+\left\{\delta_{0}\left(\mathrm{cP} P_{1} \cdot \Psi_{0}\right)-\gamma_{0} \beta_{0} E_{1}\right\}\left(\Psi_{0}\right)_{A} \\
& =\left[\begin{array}{l}
2 \\
1 \\
2
\end{array}\right]+\{(1 / 4)(8 / 3)-(3 / 4)(3)\}\left[\begin{array}{l}
1 / 3 \\
2 / 3 \\
2 / 3
\end{array}\right]=\left[\begin{array}{r}
53 / 36 \\
-2 / 36 \\
34 / 36
\end{array}\right]=\left[\begin{array}{l}
c p_{1 . x}^{\prime} \\
C p_{1 y}^{\prime} \\
C p_{1 z}^{\prime}
\end{array}\right] \\
& E_{1}^{\prime}=\gamma_{0}\left\{-\beta_{0}\left(c P_{1} \cdot \Psi_{0}\right)+E_{1}\right\}=7 / 4, \quad c p_{1}^{\prime}=7 / 4 \\
& \left(\mathrm{CP}_{2}^{\prime}\right)_{A^{\prime}}=\left[\begin{array}{r}
-1 \\
1 \\
0
\end{array}\right]+\{(1 / 4)(1 / 3)-(3 / 4)(2)\}\left[\begin{array}{l}
1 / 3 \\
2 / 3 \\
2 / 3
\end{array}\right]=\left[\begin{array}{r}
-53 / 36 \\
2 / 36 \\
-34 / 36
\end{array}\right]=\left[\begin{array}{l}
c P_{2 x}^{\prime} \\
c P_{2 y}^{\prime} \\
c p_{2 z}^{\prime}
\end{array}\right] \\
& E_{2}^{\prime}=5 / 4\{(-3 / 5)(1 / 3)+2\}=9 / 4, \quad c p_{2}^{\prime}=7 / 4
\end{aligned}
$$

Note: $\quad \mathrm{cP}_{1}^{\prime}+\mathrm{cP}_{2}^{\prime}=0, \quad \mathrm{E}_{1}^{\prime}+\mathrm{E}_{2}^{\prime}=\mathrm{e}_{\mathrm{o}}$

Standard device (App. II):

Basic direction $\Psi_{1}^{\prime}=\mathrm{CP}_{1} / C P_{1}^{\prime}=(53 / 63,-2 / 63,34 / 63) A^{\prime}$

Auxiliary direction $\Omega^{\prime}=(-2 / 3,1 / 3,2 / 3) A^{\prime}$

$$
\begin{aligned}
\Psi_{3}^{\prime}=\delta \Omega^{\prime} & =\left[\begin{array}{ccc}
53 / 63 & 2 / 63 & -34 / 63 \\
-2 / 63 & 1826 / 1827 & 17 / 1827 \\
34 / 63 & 17 / 1827 & 1538 / 1827
\end{array}\right] \cdot\left[\begin{array}{r}
-2 / 3 \\
1 / 3 \\
2 / 3
\end{array}\right] \\
& =\left[\left.\begin{array}{c}
-4988 \\
1976 \\
1121
\end{array}\right|_{A^{\prime}} / 5481\right.
\end{aligned}
$$




$$
\begin{aligned}
& E_{3}^{\prime}=\frac{e_{0}^{2}+e_{3}^{2}-e_{4}^{2}}{2 e_{0}}=13 / 8, \quad c P_{3}^{\prime}=(105)^{\frac{1}{2} / 8} \quad C P_{3}^{\prime}=c P_{3}^{\prime} \Psi_{3}^{\prime} \\
& E_{4}^{\prime}=19 / 8 \quad, \quad c p_{4}^{\prime}=(105)^{\frac{1}{2} / 8} \quad c P_{4}^{\prime}=-c P_{3}^{\prime} \\
& \quad\left(c P_{3}\right)_{A}=\left(c P_{3}^{\prime}\right)_{A^{\prime}}+\left\{\delta_{0}\left(C P_{3}^{\prime} \cdot \Psi_{0}\right)+\gamma_{0} B_{0} E_{3}^{\prime}\right\}\left(\Psi_{0}\right)_{A} \\
& E_{3}=\gamma_{0}\left\{B_{0}\left(C P_{3}^{\prime} \cdot \Psi_{0}\right)+E_{3}^{\prime}\right\}
\end{aligned}
$$

are then computable and $\left(\mathrm{CP}_{4}\right)_{A}=\left(\mathrm{CP}_{0}\right)_{A}-\left(\mathrm{CP}_{3}\right)_{A}, \quad \mathrm{E}_{4}=\mathrm{E}_{0}-\mathrm{E}_{3}$.

Some of the above steps are obviously unnecessary, but are included as a check.

Example 2. A photon $(h=1)$ of energy 4 strikes a particle $(h=2)$ of rest energy 3 and k.e. 2 at right angles, their directions on $\Sigma$ axes $A$ being

$$
\Psi_{1}=(1 / \sqrt{ } 2,1 / \sqrt{ } 2,0)_{A} \quad \Psi_{2}=(1 / \sqrt{ } 2,-1 / \sqrt{ } 2,0)_{A}
$$

The collision results in two particles $(i=3,4)$ of rest energies 2,4 respectively. The auxiliary direction $\Omega^{\prime}=(1 / \sqrt{2},-1 / 2,1 / 2)$ is chosen for location of $\Psi_{3}^{\prime}$ about the stipulated photon direction $\Psi_{1}^{\prime}$ in the $\Sigma$ ' frame of the class. Here we have

$$
\begin{array}{lllll}
e_{1}=0 & E_{1}=4 & c P_{1}=4 & \Psi_{1}=(1 / \sqrt{2}, 1 / \sqrt{ } 2,0)_{A} & c P_{1}=(2 \sqrt{2}, 2 \sqrt{2}, 0)_{A} \\
e_{2}=3 & E_{2}=5 & c P_{2}=4 & \Psi_{2}=(1 / \sqrt{ } 2,-1 / \sqrt{ } 2,0)_{A} & c P_{2}=(2 \sqrt{2},-2 \sqrt{ }, 0)_{A} \\
e_{a}=3 & E_{0}=9 & c P_{0}=4 \sqrt{ } 2 & \Psi_{0}=(1,0,0)_{A} & c P_{0}=(4 \sqrt{ }, 0,0)_{A} \\
e_{0}=7 & r_{0}=9 / 7 & B_{0}=4 \sqrt{ } / 9 & &
\end{array}
$$


Since $e_{o}>e_{S}=6, I=2$, a non-coherent system $S$ is possible. Moreover, $\Psi_{0}=X$, so the given axes are standard, and we define parallel $\Sigma^{\prime}$ axes $A^{\prime}$ in the standard configuration of Fig. 5.1.

We next compute the basic direction $\psi_{1}^{\prime}$ on axes $A^{\prime}$ :

$$
\begin{aligned}
& c p_{1 x}^{\prime}=\gamma_{0}\left(c p_{1 x}-\beta_{0} E_{1}\right)=2 \sqrt{2} / 7 \\
& E_{1}^{\prime}=\gamma_{0}\left(-\beta_{0} c p_{1 x}+E_{1}\right)=20 / 7 \\
& c p_{1}^{\prime}=\left(E_{1}^{2}-e_{1}^{2}\right)^{\frac{1}{2}}=20 / 7 \\
& a_{1 x}^{\prime}=c p_{1 x}^{\prime} / c p_{1}^{\prime}=\sqrt{ } 2 / 10 \\
& \Psi_{1}^{\prime}=\left(\sqrt{ } 2 / 10,7 \sqrt{ } 2 / 10,0_{A^{\prime}}^{\prime}\right.
\end{aligned}
$$

Applying the device of Appendix II, we define

$$
\begin{aligned}
\Omega^{\prime} & =(1 / \sqrt{ } 2,-1 / 2,1 / 2) A^{\prime} \\
D & =\left[\begin{array}{ccc}
\sqrt{ } 2 / 10 & -7 \sqrt{ } 2 / 10 & 0 \\
7 \sqrt{ } 2 / 10 & \sqrt{ } 2 / 10 & 0 \\
0 & 0 & 1
\end{array}\right]
\end{aligned}
$$

so $\Psi_{3}^{\prime}=D\left(\Omega^{\prime}\right)_{A^{\prime}}$ is the $\Sigma^{\prime}$ direction of 3 on axes $A^{\prime}$.

Since $I=2$, we have uniquely by $\$ 18$

$$
\begin{aligned}
E_{3}^{\prime} & =\left(e_{0}^{2}+e_{3}^{2}-e_{4}^{2}\right) / 2 e_{0}=37 / 14 \\
C P_{3}^{\prime} & =\left(E_{3}^{\prime 2}-e_{3}^{2}\right)^{\frac{1}{2}}=3 \sqrt{6} 65 / 14 \\
C P_{3}^{\prime} & =C P_{3}^{\prime}\left(\Psi_{3}^{\prime}\right)_{A}^{\prime}
\end{aligned}
$$


From this we obtain the $\Sigma$ energy

$$
E_{3}=\gamma_{0}\left(\beta_{0} c p_{3 x}^{\prime}+E_{3}^{\prime}\right)
$$

and the coordinates of $\mathrm{cP}_{3}$ on $\Sigma$ axes $A$ :

$$
c p_{3 x}=\left(c p_{3 x^{\prime}}^{\prime}+B_{0} E^{\prime}\right) \quad c p_{3 y}=c p_{3 y}^{\prime} \quad c p_{3 z}=c p_{3 z}^{\prime}
$$

The last two examples are quite arbitrary. Compton collision is ordinarily treated in the rest frame of the target (531).

For instance, in the present example, we should find

$$
\begin{aligned}
& c P_{1}^{\prime}=c P_{1}+\left\{\left(\gamma_{2}-1\right)\left(c P_{1} \cdot \Psi_{2}\right)-\gamma_{2} \beta_{2} E_{1}\right\} \Psi_{2}=(-2 \sqrt{2} / 3,14 \sqrt{2} / 3,0) \\
& c p_{1}^{\prime}=20 / 3 \quad \Psi_{1}=(-\sqrt{ } 2 / 10,7 \sqrt{2} / 10,0) \text { for the photon in the rest }
\end{aligned}
$$

frame of the target.

23. The Q-value. The Q-value of a (proposed) transmutation

$$
A\left(e_{h}\right) \rightarrow S\left(e_{i}\right)
$$

is defined as the intrinsic difference

$$
Q=e_{a}-e_{s}
$$

in the total proper energies of the two systems. Thus the energy conservation equation in $\Sigma$, 


$$
e_{a}+k_{a}=E_{o}=e_{s}+k_{s}
$$

is expressible in the form

$$
k_{a}+Q=k_{s}
$$

emphasizing that, in the conversion of $A$ into $S$, the "loss" in proper energy must balance the "gain" in k.e. This also makes obvious the invariance of the k.e. difference of two systems of the same class under an arbitrary Lorentz transformation. Moreover, the invariantly expressed threshold condition

$$
e_{0} \geq e_{s}
$$

is equivalent to $E_{0} \equiv e_{0} \gamma_{0} \geq e_{s} \gamma_{0}$. Since $E_{0}=e_{a}+k_{a}$, this may be written in the form

$T(\Sigma)$

$$
k_{a} \geq(-Q)+\left(\gamma_{0}-1\right) e_{s}
$$

Thus the threshold condition in $\Sigma$ states that the total k.e. $\mathrm{k}_{\mathrm{a}}$ of the initial system must suffice to supply any proper energy increase, plus the k.e. of a particle of proper energy $e_{s}$ riding at its (CM).

In the $\Sigma^{\prime}$ frame of the class, the corresponding transmutation $A^{\prime} \rightarrow S^{\prime}$ satisfies the conservation law

$$
e_{a}+k_{a}^{\prime}=e_{o}=e_{s}+k_{s}^{\prime}
$$


or

$$
\mathbf{k}^{\prime}+Q=k_{s}^{\prime}
$$

Here the threshold condition $e_{0} \geq e_{s}$ is equivalent to

$$
T\left(\Sigma^{\prime}\right) \quad k_{a}^{\prime} \geq(-Q)
$$

$$
\text { since } e_{0}=e_{a}+k_{a}^{\prime} \text {. }
$$

The conservation equation (2),

$$
e_{a}+k_{a}=e_{0}+k_{0}=e_{s}+k_{s}
$$

is formally like the law of constant potential plus kinetic energy in mechanics. Indeed, one may think of a transmutation as a procesis which raises $e_{a}$ to a maximal value $e_{0}$, and then drops it to a final level $e_{s}$, where $Q=e_{a}-e_{s}$ is the net "potential drop," as indicated in Figure 1.

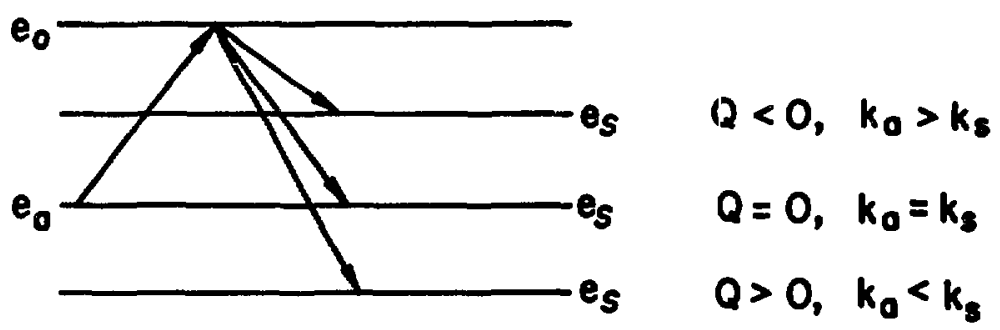

Figure 1.

Rest Mass as "Potential"

The transmutation $A \rightarrow S$ is said to be elastic in case the total kinetic energy is conserved, as well as $E_{0}$ and $P_{0^{\circ}}$ Such a change is therefore one for which we have the additional stipulation $k_{a}=k_{s}$, or 
equivalently $Q=0$. Hence the elastic nature of a collision is invariant under Lorentz transformation, and is always possible since $e_{a}<e_{0}$ and $e_{s}=e_{a}$

In practice $e_{a}=e_{s}$ signifies the identity of the individual proper energies of $A$ and $S$, and when $H=2=I$, the two participating particles preserve their individual energies and absolute momenta in the $\Sigma$ ' frame of the class, as required by Theorem 18.1 .

24. Decay. By a "decay" we mean any transmutation of form

$$
A\left(e_{0}\right) \rightarrow S\left(e_{1}, \ldots, e_{I}\right) ; \quad I \geq 2
$$

in which a single material particle is converted into a system of two or more particles. Here, the proper energy of the decaying particle is necessariiy the critical energy of its class $\left\{\mathrm{cP}_{0}, \mathrm{E}_{0}\right\}$, and the necessary condition $e_{o} \geq e_{s}$ becomes simply

T(D) $\quad Q \geq 0$

Thus the decay is prohibited if $Q<0\left(e_{0}<e_{s}\right)$ regardless of the k.e. of $e_{0}$ (why is this consistent with (23.5)?). If $Q=0$, decay is possible iff all $e_{i}>0$ with a trivial coherent result. If $Q>0$ (the only case of physical interest) the decay is always possible since we have stipulated $I \geq 2$.

The parameters $\gamma_{0}, \beta_{0}, \Psi_{0}$ of the transformation to the $\Sigma^{\prime}$ frame of the class are those of the decaying particle; in particular

$$
\gamma_{0}=E_{0} / e_{0}, \quad \beta_{0}=c p_{0} / E_{0}
$$


Moreover, the $\Sigma '$ frame is the rest frame of the particle, in which it appears stationary, with rest energy $e_{0}$, and some intrinsic life time $T^{\prime}$. We recall (56) that its apparent life span in $\Sigma$ is $T=\gamma_{0} T^{\prime}$, during which it travels a distance $\gamma_{0} \beta_{0} c T^{\prime}$ in the direction $\Psi_{0}(\xi 6)$.

In $\Sigma '$, the decay appears as an "explesion" of a particle at rest, with a conversion of proper to kinetic energy indicated by the equation

$$
0+Q=k_{s}^{\prime}
$$

Some forbidden decays. (a) For a bare nucleus $\left({ }_{Z}^{A}\right)$ of charge $+Z q$ containing A nucleons ( $Z$ protons $\mathrm{p}^{+}, \mathrm{N}=\mathrm{A}-\mathrm{Z}$ neutrons $\mathrm{n}^{\circ}$ ) the decay

$$
\left(\begin{array}{l}
\mathrm{A} \\
\mathrm{Z}
\end{array} \mathrm{Zp}^{+}+\mathrm{Nn}^{\mathrm{O}}\right.
$$

is impossible since $Q=e\left(z^{A}\right)-\left\{Z e\left(p^{+}\right)+N e\left(n^{0}\right)\right\}<0$. Here $-Q$ is called the binding energy of the nucleus.

(b) For the neutral atom $\left[Z_{Z}^{A}\right]$ with $z$ electrons $\varepsilon^{-}$in ground state about the nucleus, the decay

$$
\left[\mathrm{Z}_{\mathrm{Z}}^{\mathrm{A}}\right]+\left(\begin{array}{c}
\mathrm{A}_{\mathrm{Z}} \\
)
\end{array}+\mathrm{E}^{-}\right.
$$

is forbidden, since $Q=e\left[Z_{Z}^{A}\right]-\left\{e\left(\frac{A}{Z}\right)+Z e\left(\varepsilon^{-}\right)\right\}<0,-Q$ being the binding energy of the electrons. In nuclear reactions, this is ignored. For example in (a) one would take $Q \simeq e\left[Z^{A}\right]-\left\{Z_{e}\left[\begin{array}{l}1 \\ 1\end{array}\right]+N\left(n^{O}\right)\right\}$, where $\left[\begin{array}{l}1 \\ 1\end{array}\right]$ denotes the neutral ${ }_{1}^{1}$ atom. Table IV gives a few neutral atom proper energies in "atomic mass units" (AMU). From this one may verify the binding energy (2.225 MeV of the deuteron $\left[{ }_{1}^{2}\right]={ }_{1}^{2} \mathrm{H}$. 
(c) The decay $\mathbf{p}^{+} \rightarrow \mathrm{n}^{0}+\varepsilon^{+} \div \nu_{\varepsilon}$ of a free proton into a neutron, positron and neutrino is forbidden by the $Q$-value $Q=-1.8 \mathrm{MeV}$ (Table III), although this is the basis of positron decay in unstable nuclei.

(d) An electron decay $\varepsilon^{-}+\varepsilon^{-}+\gamma$ has $Q=0$ but violates the requirement that all product proper energies be positive.

(e) The process $\mathrm{p}^{+} \rightarrow \mathrm{e}^{+}$has $\mathrm{Q}>0$, but does not produce at least two particles.

Since we regard a transmutation $A \rightarrow S$ as possible iff $A, S$ are in the same class, its impossibility obviously implies the impossibility of $S+A$, although the latter may be true for apparently different reasons. Consider the reverse of the transmutations in (d) and (e).

25. Decay into two particles. In a decay

$$
A\left(e_{0}\right) \rightarrow S\left(e_{1}, e_{2}\right)
$$

where $k_{s}^{\prime}=Q=e_{0}-\left(e_{1}+e_{2}\right)>0$, the products emerge in opposite directions in the $\Sigma^{\prime}$ frame of the class, with the unique energies $E_{i}^{\prime}, k_{i}^{\prime}$ given in (18.2), (18.9). The relation between the systems $S\left(e_{1}, e_{2}\right) \sim S^{\prime}\left(e_{1}, e_{2}\right)$ is illustrated by Fig. 20.1. If $e_{0}$ is at rest in $\Sigma$, the frames $\Sigma, \Sigma$ ' coincide, and all parameters of $S$ are of course those of $S^{\prime}$.

I. In the sinplest case, one has $e_{1}=e_{2} \equiv e$, as in Note 20.3 , so that

$$
\begin{aligned}
& k_{s}^{\prime}=Q=e_{0}-2 e, \text { and for } i=1,2, \\
& k_{i}^{\prime}=Q / 2, \quad E_{i}^{\prime}=e_{o} / 2, \quad C P_{i}^{\prime}=\left(\left(e_{0} / 2\right)^{2}-e^{2}\right)^{\frac{1}{2}} .
\end{aligned}
$$




$$
K^{0} \rightarrow \pi^{-}+\pi^{+}
$$

$Q=218.6 \mathrm{MeV}$, and each pion has k.e. $109.3 \mathrm{MeV}$ in $\Sigma^{\prime}$.

In particular, when $e_{1}=e_{2}=0$, the decay involves a total conversion of the rest energy $e_{o}$ into kinetic energy

$$
k_{S}^{\prime}=Q=e_{0}
$$

with $k_{i}^{\prime}=E_{i}^{\prime}=c p_{i}^{\prime}=e_{0} / 2$. This is the case for which we have the simple result of (20.4),

$$
\sin \sigma / 2=e_{0} / 2\left(E_{1} E_{2}\right)^{\frac{1}{2}} \geq e_{o} / E_{0}=1 / \gamma_{0}
$$

for the angle $\sigma$ of separation between the two lines of flight in $\Sigma$.

For example, the decay in flight

$$
\pi^{0} \rightarrow \gamma+\gamma
$$

of a $135 \mathrm{MeV}$ (k.e.) neutral pion yields photons with a minimum angle of separation of $60^{\circ}$ in $\Sigma$, each having energy $E_{i}=c p_{i}=135 \mathrm{MeV}$. (cf. (20.3))

A second instance is provided by the decay of "parapositroneum:" $\left\{\varepsilon^{+} \uparrow, \varepsilon^{-}+\right\} \rightarrow \gamma \uparrow+\gamma \downarrow$. Neglecting its binding energy, each photon in $\Sigma^{\prime}$ has energy $h \nu_{i}^{\prime}=e(\varepsilon)=.5110 \mathrm{MeV}$, and wavelength $\lambda_{i}^{\prime}$ (by definition) the Compton wavelength of the electron (\$1).

Note. Although a free positron $\varepsilon^{+}$is stable, it comes to rest locally when liberated (as in pair production and positron emission) in the presence of 
matter, and may then combine with an electron $\varepsilon^{-}$to form a very unstable "double star" complex $\left\{\varepsilon^{+}, \varepsilon^{-}\right\}$called positroneum. When the component spins are opposite (the usual case) the result is parapositroneum, with the decay mode above, the photon spins also being opposite. Spin conservation is indicated by the equation $\frac{1}{2}-\frac{1}{2}=0=1-1$. The alternative result is "ortho positroneum," with the decay $\left\{\varepsilon^{+} \uparrow, \varepsilon^{-} \uparrow\right\} \rightarrow \gamma \uparrow+\gamma \uparrow+\gamma \downarrow$, and spin conservation $\frac{1}{2}+\frac{2}{2}=1=1+1-1$.

II. In another important case, one has $e_{1}>0, e_{2}=0$, with $k_{s}^{\prime}=Q=$ $e_{0}-e_{1}>0$. Here, the formula (18.9) for the k.e. of $e_{1}$ in $\Sigma$ ' becomes

$$
\mathrm{k}_{\mathrm{i}}^{\prime}=\mathrm{Q} \rho, \quad \rho=\mathrm{Q} / 2 \mathrm{e}_{\mathrm{o}}
$$

and the relations

$$
E_{i}^{\prime}=e_{1}+k_{1}^{\prime}, \quad c P_{i}^{\prime}=E_{2}^{\prime}=k_{2}^{\prime}=Q(1-\rho)
$$

provide an easy way of obtaining the remaining parameters of S'. Since $Q=e_{0}-e_{1}<e_{0}$, it is clear that $\rho<\frac{1}{2}$ and hence

$$
k_{1}^{\prime}<k_{s}^{\prime} / 2<k_{2}^{\prime}
$$

while the energy formulas

$$
\begin{aligned}
& E_{1}^{\prime}=\left(e_{0} / 2\right)\left\{1+\left(e_{1} / e_{0}\right)^{2}\right\} \\
& E_{2}^{\prime}=\left(e_{0} / 2\right)\left\{1-\left(e_{1} / e_{0}\right)^{2}\right\}
\end{aligned}
$$


show that

$$
E_{1}^{\prime}>e_{0} / 2>E_{2}^{\prime}
$$

As noted in $\$ 18$, such inequalities obtain whenever $e_{1}>e_{2}$.

The $E_{2}^{\prime}$ equation implies the relation

$$
e_{0}=k_{2}^{\prime}+\left(k_{2}^{\prime 2}+e_{1}^{2}\right)^{\frac{1}{2}}
$$

which may be used to compute the rest energy of the decaying particle from that of the material product particle, and the energy of the accompanying photon.

The decay modes of some of the "fundamental particles" (Table III) fall under Case II, for example, in the decay

$$
\Sigma^{0}+\Lambda^{0}+\gamma
$$

Taking $e_{0}=1193, e_{1}=1116$, we have $Q=77, \rho=.0323, k_{1}^{\prime}=2.487$, $E_{1}^{\prime}=1118.5, c p_{i}^{\prime}=E_{2}^{\prime}=k_{2}^{\prime}=74.5(\mathrm{a} 11 \mathrm{MeV})$.

In a nuclear decay of this type, $Q=e_{0}-e_{1}$ may be thought of as the difference in energy levels of the "same" nucleus, having rest energies $e_{0}>e_{1}$ in the two states. In such a photon emission it is interesting to compare the emitted photon wavelength $\lambda_{2}^{\prime}=c / v_{2}^{\prime}$ with the "normal" wavelength $\lambda_{0}=\mathrm{c} / \nu_{0}$, where by definition $\mathrm{h} \nu_{0}=Q$ is the difference in energy levels. Dividing the latter equation by $h v_{2}^{\prime}=k_{2}^{\prime}=Q(1-\rho)$ yields $\lambda_{2}^{\prime} / \lambda_{0}=v_{0} / v_{2}^{\prime}=1 /(1-p)$ and therefore 


$$
z^{\prime}=\left(\lambda_{2}^{\prime}-\lambda_{0}\right) / \lambda_{0}=\rho /(1-\rho)=k_{1}^{\prime} / k_{2}^{\prime}=Q /\left(Q+2 e_{1}\right)
$$

is the "red shift due to recoil" in $\Sigma$ '.

III. When $e_{1}>e_{2}>0$, the general formulas of $\$ 18$ are required. The decay modes $\Xi^{-}+\Lambda^{0}+\pi^{-}$and $\Sigma^{-} \rightarrow n^{0}+\pi^{-}$of Table III, and the classical nuclear emission of $\alpha$ particles $\left({ }_{2}^{4} \mathrm{He}\right)$ are of this kind.

Example 1. A particle of rest energy 3, traveling on $0 x$ with k.e. 2 decays in flight into two particles each of rest energy 1 , the first of which is emitted in $\Sigma^{\prime}$ at $60^{\circ}$ with $0 X^{\prime}$. Then for $A\left(e_{0}\right)+5\left(e_{1}, e_{2}\right), e_{0}=3, k_{0}=2$, $E_{0}=5, c p_{0}=4, \gamma_{0}=5 / 3, \beta_{0}=4 / 5$

$e_{i}=1, \quad E_{i}^{\prime}=3 / 2, c p_{i}^{\prime}=\sqrt{ } 5 / 2, \quad i=1,2$

$E_{1}=5 / 3(4 / 5 \cdot \sqrt{5} / 2 \cdot 1 / 2+3 / 2)=(15+2 \sqrt{5}) / 6$

$E_{2}=5 / 3(4 / 5 \cdot \sqrt{5} / 2 \cdot(-1 / 2)+3 / 2)=(15-2 \sqrt{5}) / 6$

Note that this falls under Case III of Note 20.3.

Example 2. The particle $\left(h=0, e_{0}=3, k_{0}=2\right)$ of Ex. 1 has direction $\Psi_{0}=(2 / 3,2 / 3,1 / 3)_{A}$ on $\Sigma$ axes $A$, and decays in flight into the same two particles of rest energy $e_{1}=e_{2}=1$. If, as we shall assume, the decay product 1 is emitted isotropically in the rest frame of the decaying particle, the "basic direction of emission" is ours to choose, and we shall do so in two different ways in the two methods (I), (II) (See Note 22.1 and App. II). We suppose the auxiliary direction $\Omega^{\prime}=(2 / 7,6 / 7,3 / 7)$ in either case. Here we have 


$$
\begin{aligned}
e_{0}=3 \quad E_{0} & =5 \quad c P_{0}=4 \quad \Psi_{0}=(2 / 3,2 / 3,1 / 3)_{A} \quad c P_{0}=(8 / 3,8 / 3,4 / 3)_{A} \\
\gamma_{0} & =5 / 3 \quad \beta_{0}=4 / 5
\end{aligned}
$$

The proposed system $S$ has $e_{S}=2<e_{0}=3, I=2$, so non-coherent decay is possible.

As before, we have for $S^{\prime}$ in $\Sigma^{\prime}$,

$$
e_{i}=1 \quad E_{i}^{\prime}=3 / 2 \quad c p_{i}^{\prime}=\sqrt{5} / 2, \quad i=1,2 .
$$

We now consider the two methods of Note 22.1.

(I) We choose $\Sigma^{\prime}$ axes $A^{\prime}$ parallel to the given $\Sigma$ axes $A$ and select $\mathrm{X}^{\prime}$ as the basic direction $\Psi^{\prime}$ (Transformation of $\mathrm{A}^{\prime}$ to $\mathrm{A}^{\prime}$ is unnecessary). We take the $\Sigma^{\prime}$ direction of 1 to be $\Psi_{1}^{\prime}=\Omega^{\prime}=(2 / 7,6 / 7,3 / 7){ }_{A}^{\prime}$, and so have

$$
E_{1}^{\prime}=3 / 2, \quad \mathrm{CP}_{1}^{\prime}=(\sqrt{5} / 2) \Psi_{1}^{\prime} \text { on axes } A^{\prime} .
$$

Then one computes $\mathrm{E}_{1}$ and $\left(\mathrm{cP}_{1}\right)_{A}$ by using the vector form

$$
\begin{aligned}
& \left(\mathrm{CP}_{1}\right)_{A}=\left(\mathrm{CP}_{1}^{\prime}\right)_{A^{\prime}}+\left\{\left(\gamma_{0}-1\right)\left(\mathrm{CP}_{1} \cdot \Psi_{0}\right)+\gamma_{0} \beta_{0} E^{\prime}\right\}\left(\Psi_{0}\right)_{A} \\
& E_{1}=\gamma_{0}\left\{\beta_{0}\left(\mathrm{CP}_{1} \cdot \Psi_{0}\right)+E_{1}^{\prime}\right\}
\end{aligned}
$$

The inner product required is $\left(\mathrm{CP}_{i} \cdot \Psi_{0}\right)=19 \sqrt{5 / 42}$ and the energy is $\mathrm{E}_{1}=38 \sqrt{5 / 63}+5 / 2$. We omit computation of $\left(\mathrm{cP}_{1}\right)_{A}$.

(II) We obtain a set of standard axes $S$ in $\Sigma$ by using the rotation $\delta$ of App. I, Corollary 1 , with $\delta_{1} X=\Psi_{0}$ and associated matrix 


$$
D=\left[\begin{array}{lll}
2 / 3 & -2 / 3 & -1 / 3 \\
2 / 3 & 11 / 15 & -2 / 15 \\
1 / 3 & -2 / 15 & 14 / 15
\end{array}\right]
$$

Defining standard $\Sigma^{\prime}$ axes $S^{\prime}$ paraliel to $S$, we select $\bar{X}$ ' as the basic direction of emission in $\Sigma^{\prime}$ and define

$$
\Psi_{1}^{\prime}=\Omega^{\prime}=(2 / 7,6 / 7,3 / 7)_{S}
$$

(It is not of course the same absolute direction as that in (I).)

Thus $\mathrm{E}_{1}^{\prime}=3 / 2 \quad \mathrm{CP}_{1}^{\prime}=(\sqrt{5} / 2) \Psi_{1}^{\prime}$ on $S^{\prime}$. Hence from the simple (cPE) transformation (10.2) we find

$$
\begin{aligned}
& \mathrm{E}_{1}=4 \sqrt{5} / 21+5 / 2 \\
& \mathrm{cP}_{1}=(5 \sqrt{5} / 21+2,3 \sqrt{ } 5 / 7,3 \sqrt{ } 5 / 14)_{S}
\end{aligned}
$$

on standard $\Sigma$ axes $S$. (Note the check: $E_{1}^{2}-\left(\mathrm{cP}_{1}\right)^{2}=1=\mathrm{e}_{1}^{2}$.)

The components of $\mathrm{CP}_{1}$ relative to the original $\Sigma$ axes $A$ are then obtained from

$$
\left(\mathrm{cP}_{1}\right)_{A}=\mathrm{D}\left(\mathrm{cP}_{1}\right)_{S}
$$

as in Theorem 1 (b) of App. I. The matrix D is used here only. 26. Decay into three particles. In a decay

$$
A\left(e_{0}\right) \rightarrow S\left(e_{1}, e_{2}, e_{3}\right)
$$


where $k_{S}^{\prime}=Q=e_{0}-\left(e_{1}+e_{2}+e_{3}\right)>0$, the $\Sigma$ ' energies of $S^{\prime}$ are not unique, and $a:$ shown in 521 , any one of its particles (say $i=1$ ) may have for its k.e. values on the range

$$
0<k_{1}^{\prime}<k_{j}^{\prime} \equiv \frac{k_{s}^{\prime}}{e_{0}}\left(e_{K}+\frac{1}{2} k_{s}^{\prime}\right), \quad k_{s}^{\prime}=Q
$$

where $e_{K}=e_{2}+e_{3}$. (See Corollary 21.2 for end points.)

For example, the $\Sigma^{\prime}$ energy range of any one of the three photons produced in orthopositronium decay (525) is $0<\mathrm{k}_{1} \leq .511 \mathrm{MeV}$.

Perhaps the most notable instance is the decay

$$
\mathrm{n}^{0}+\varepsilon^{-}+\mathrm{p}^{+}+\bar{\nu}_{E} \quad(\mathrm{Q}=.783 \mathrm{MeV})
$$

of the free neutron (mean life $932 \mathrm{sec}$ )

Thus the electron should have a k.e. range

$$
0 \leq k_{1}^{\prime}<\frac{.783}{939.553}(938.651)=.7822 \mathrm{MeV}
$$

and this is observed experimentally.

Note 1. A decay of form $n^{0}+\varepsilon^{-}+p^{+}$has the same positive Q-value and is also mechanically possible, but would result in a unique k.e. $k_{j}=k_{j}$ for the electron, in conflict with experiment, and would violate spin conservation, since $\pm \frac{1}{2} \neq \pm \frac{1}{2} \pm \frac{1}{2}$ for any choice of signs. (Hence the "invention" of the neutrino by Pauli, and it "discovery" by Reines and Cowan.) 
Note 2. Neutron decay is the basic process in electron emission from unstable nuclei ( $\beta$-decay),

$$
\left(\begin{array}{c}
A \\
z
\end{array}\right) \varepsilon^{-}+\left(\begin{array}{c}
A \\
z+1
\end{array}\right)+\bar{v}_{\varepsilon}
$$

e.g. in the decay of the triton

$$
\left({ }_{1}^{3} \mathrm{H}\right)+\varepsilon^{-}+\left({ }_{2}^{3} \mathrm{He}\right)+\bar{v}_{\varepsilon}, Q=.0182 \mathrm{MeV}
$$

Note 3. The analogous nuclear positron emission

$$
\left(\begin{array}{l}
A \\
Z
\end{array}\right)+\varepsilon^{+}+\left(\begin{array}{c}
A \\
Z-1
\end{array}\right)+v_{\varepsilon}
$$

is observed, although the process is forbidden to the free proton. An example is the transition

$$
\left({ }_{6}^{11} \mathrm{C}\right) \rightarrow \varepsilon^{+}+\left({ }_{5}^{11} \mathrm{~B}\right)+v_{\varepsilon^{\prime}}, \quad Q=.96 \mathrm{MeV}
$$

Note here that, for such decays, "adding" $z$ electrons to each side results in the neutral atom "reaction"

$$
\left[\begin{array}{c}
A \\
Z
\end{array}\right] \rightarrow \varepsilon^{+}+\varepsilon^{-}+\left[\begin{array}{c}
A \\
Z-1
\end{array}\right]+\nu_{\varepsilon}
$$

so that $Q \simeq e\left[\begin{array}{l}A \\ Z\end{array}\right]-2 e(\varepsilon)-e\left[\begin{array}{c}A \\ Z-1\end{array}\right]$

where $e(\varepsilon)=.511 \mathrm{MeV}$. 
27. Collisions with target at rest (TAR). Every transmutation of form $A\left(e_{h}\right)+S\left(e_{i}\right)$ in which $A$ consists of a single particle $(H=1)$ may be regarded as a "decay." All others, with $\mathrm{H} \geq 2$, are called "collisions," and we shall consider only those with $H=2$, of the form

$$
A\left(e_{1}, e_{2}\right)+S\left(e_{i}\right) ; \quad Q=e_{a}-e_{S}
$$

where particle 1 will be called the "projectile," and 2 the "target."

We study first the important special case in which the target is a (necessarily) material particle at rest in its own rest frame $\Sigma$, with the projectile moving toward it on "collision course." As a common tasis for the following sections dealing with such collisions, we consider as given an initial system $A\left(e_{1}, e_{2}\right)$, with particle parameters

$$
\begin{aligned}
& e_{1} \geq 0 \quad k_{1}>0 \quad E_{1}=e_{1}+k_{1} \quad c p_{1}=\left(E_{1}^{2}-e_{1}^{2}\right)^{\frac{1}{2}} \quad c P_{1}=c p_{1} \Psi_{1} \\
& e_{2}>0 \quad k_{2}=0 \quad E_{2}=e_{2} \quad c p_{2}=0 \quad c P_{2}=0
\end{aligned}
$$

and hence with the totals

$$
e_{a}=e_{1}+e_{2} \quad k_{a}=k_{1} \quad E_{0}=E_{1}+e_{2} \quad c p_{0}=c p_{1} \quad c P_{0}=c P_{1}
$$

The critical energy of the class $\left\{\mathrm{E}_{\mathrm{o}}, \mathrm{CP} \mathrm{P}_{\mathrm{o}}\right\}$ to which $\mathrm{A}$ belongs is therefore $e_{0}=\left(E_{0}^{2}-c p_{0}^{2}\right)^{\frac{1}{2}}$, i.e.,

$$
e_{0}=\left(e_{1}^{2}+e_{2}^{2}+2 e_{2} E_{1}\right)^{\frac{1}{2}}=\left(e_{a}^{2}+2 e_{2} k_{1}\right)^{\frac{1}{2}}=e_{a}\left(1+\frac{2 e_{2} k_{1}}{e_{a}^{2}}\right)^{\frac{1}{2}}>e_{a}
$$


The class parameters, on which the Lorentz transformation to the $\Sigma$ ' frame of the class is based are then

$$
\gamma_{0}=E_{0} / e_{0}, \quad \beta_{0}=c p_{0} / E_{0}, \quad \gamma_{0} \beta_{0}=c p_{0} / e_{0}, \quad \Psi_{0}=\Psi_{1}
$$

The basic direction for emission of products $e_{i}$ is $\Psi_{1}$, which coincides with the direction $\Psi_{0}$ of $\Sigma^{\prime}$ in $\Sigma$, and hence with the $X$-axis of standard axes $S$.

The proper energies $e_{i} \geq 0$, of sum $e_{s}=\sum e_{i}$, being stipulated for the proposed system S, it appears fron (4) that the invariantly expressed necessary condition $e_{0} \geq e_{s}$ assumes, in the target rest frame $\Sigma$, the form

$$
k_{a}=k_{1} \geq \frac{e_{a}+e_{s}}{2 e_{2}}(-Q) \equiv k_{T}
$$

where $Q=e_{a}-e_{s}$, and $k_{T}$ is the (k.e.) "threshold" for the reaction.

Since the inequality $e_{0} \geq e_{s}$ is here strictly equivalent to $k_{1} \geq k_{T}$, we may interpret the results of Theorem 22.3 in the convenient form of Theorem 1. The proper energies $e_{i}$ of $S$ being stipulated, then I. when $Q<0\left(e_{a}<e_{s}\right), A \rightarrow S$ is impossible unless $k_{1} \geq k_{T}$;

(a) if $k_{1}=k_{T}\left(e_{0}=e_{S}\right), A \rightarrow S$ is possible iff all $e_{i}>0$. (Example: fusion)

(b) if $k_{1}>k_{T}\left(e_{0}>e_{S}\right), A \rightarrow S$ is possible iff $I \geq 2$;

II. when $Q \geq 0\left(e_{\mathrm{a}} \geq e_{\mathrm{s}}\right)$, then $k_{T} \leq 0$, and its value is irrelevant, since $k_{1}>0 \geq k_{T}$ and $e_{0}>e_{s}$. Hence $A \rightarrow S$ is possible iff $I \geq 2$. (Example: elastic collision, $Q=0$.)

We observe, if $Q<0$, that $k_{T}>(-Q)$ necessarily. 
In fact, $k_{T}=\frac{e_{a}+e_{s}}{2 e_{2}}(-Q)>\frac{2 e_{a}}{2 e_{2}}(-Q) \geq(-Q)$.

In general, energy conservation requires

$$
e_{a}+k_{1}=E_{o}=e_{s}+k_{s}
$$

or

$$
k_{1}+Q=k_{s}
$$

so that

$$
k_{s} \gtreqless k_{1} \text { as } Q \gtreqless i \text {. }
$$

In the $\Sigma^{\prime}$ frame of the class, the system $A^{\prime}\left(e_{1}, e_{2}\right)$ belongs to the class $\left\{0, e_{0}\right\}$, and, by (4) and Theorem 18.1, has the energies $E_{h}^{\prime}=\left(e_{h}^{2}+e_{2} E_{1}\right) / e_{0}$, or more simply

$$
E_{2}^{\prime}=e_{2} E_{0} / e_{0}=e_{2} \gamma_{0}, \quad E_{i}^{\prime}=e_{0}-e_{2} \gamma_{0}
$$

The oppositely directed vectors $\mathrm{CP}_{h}^{\prime}$ have the equal magnitudes

$$
C p_{h}^{\prime}=\left(E_{2}^{\prime 2}-e_{2}^{2}\right)^{\frac{1}{2}}=\gamma_{0} \beta_{0} e_{2}=\beta_{0} E_{2}^{\prime}
$$

The formula $E_{2}^{\prime}=e_{2} \gamma_{0}$ reflects the physically obvious fact that the target has the same speed in $\Sigma^{\prime}$ as the $(\mathrm{CM})$ of $\mathrm{A}$ in $\Sigma$. This accounts for the relations

$$
\gamma_{0} B_{0} E_{2}^{\prime}=\gamma_{0} C P_{2}^{\prime}, \quad \rho_{2}^{\prime}=1
$$

and the position of $0_{2}^{\prime \prime}$ on the ellipsoid $E$ in Fig. 1, showing the relation between the initial systems $A, A^{\prime}$. 


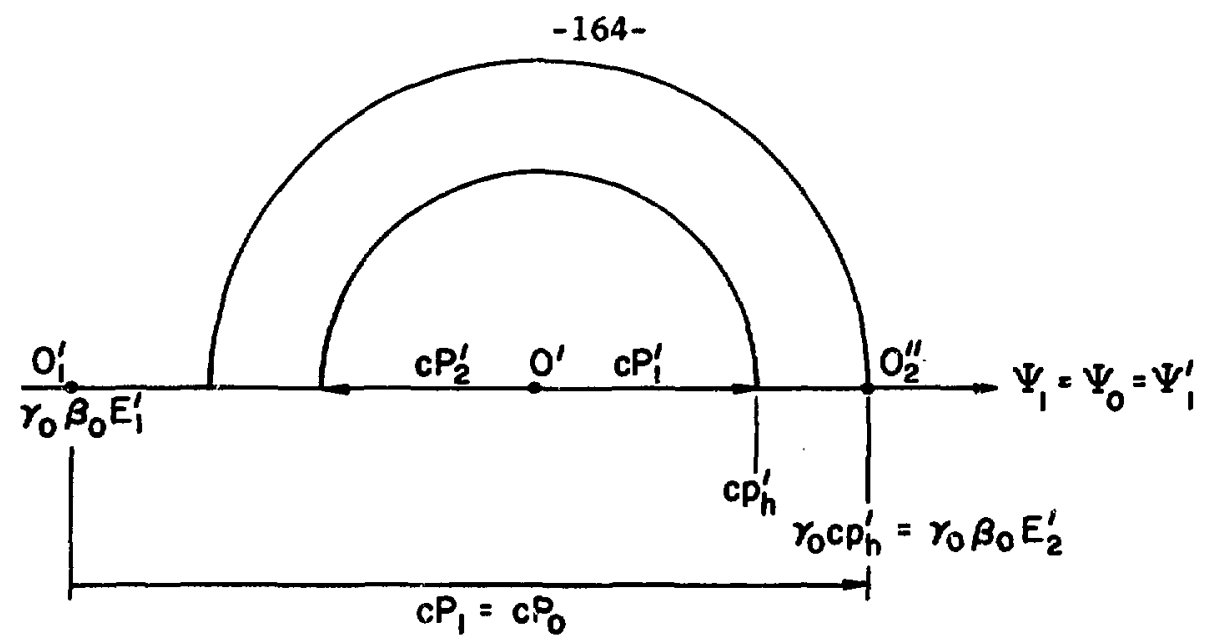

Fig. $1\left(e_{1}>e_{2}\right)$

The initial system $A$.

It may be noted here that $o_{i}^{\prime}$ falls (I) inside, (II) on, or (III) outside the ellipse $E$ according as

$$
e_{1} \leqq e_{2}
$$

i.e., in case of a projectile of proper mass "lighter" than, equal to, or "heavier" than that of the target. To see this we need only note that, in the present instance,

$$
O_{1}^{\prime} O^{\prime}=\gamma_{0} B_{0} E_{1}^{\prime} \leqq \gamma_{0} C_{1}^{\prime} P_{1}^{\prime}=0^{\prime} O_{2}^{\prime \prime}=\gamma_{0} B_{0} E_{2}^{\prime}
$$

according as $E_{1} \lesseqgtr E_{2}^{\prime}$, and the remark follows at once from the equation

$$
E_{1}^{2}-e_{1}^{2}=C p_{1}^{2}=C p_{2}^{2}=E_{2}^{2}-e_{2}^{2}
$$

Note 1. There are good reasons for considering first, in some detai1, collisions in the rest frame of the target, aside from their greater simplicity. (a) In many physical collisions, the target may be assumed essentially at rest in the laboratory frame $\Sigma$. (b) If the target is a material particle 
moving with constant velocity $v_{2} \neq 0$ in $\Sigma$, a preliminary Lorentz transformation based on $v_{2}$ will carry the colliding system into a frame in which the target is at rest, and to which the simpler theory applies. (c) The "general" methods of $\$ 34$ really require the target to be in motion, with a well-defined direction $\Psi_{2}$, specializing to the TAR case only in a limiting sense.

Example 1. A particle of rest energy 1, traveling with k.e. 4 on $0 \mathrm{X}$, strikes a motionless particle of rest energy 2 . The initial system $A$ has parameters and totals

$$
\begin{array}{lllll}
\mathrm{e}_{1}=1 & \mathrm{k}_{1}=4 & \mathrm{E}_{1}=5 & c p_{1}=2 \sqrt{6} & c P_{1}=(2 \sqrt{6}, 0,0) \\
\mathrm{e}_{2}=2 & \mathrm{k}_{2}=0 & E_{2}=2 & c p_{2}=0 & c P_{2}=(0, c, 0) \\
e_{\mathrm{a}}=3 & \mathrm{k}_{\mathrm{a}}=4 & \mathrm{E}_{0}=7 & c p_{0}=2 \sqrt{6} & c P_{0}=(2 \sqrt{6}, 0,0)
\end{array}
$$

The critical energy of its class $\left\{\mathrm{E}_{0}, \mathrm{CP} \mathrm{P}_{0}\right\}$ is $\mathrm{e}_{0}=\left(\mathrm{e}_{\mathrm{a}}^{2}+2 \mathrm{e}_{2} \mathrm{k}_{1}\right)^{\frac{1}{2}}=5$, and the pararneters governing the transformation to the $\Sigma$ ' frame of the class are

$$
\gamma_{0}=E_{0} / e_{0}=7 / 5 \quad \beta_{0}=c p_{0} / E_{0}=2 \sqrt{6} / 7
$$

The parameters of $A^{\prime}$ in $\Sigma^{\prime}$ (if required) are

$$
\begin{aligned}
& E_{2}^{\prime}=e_{2} \gamma_{0}=14 / 5 \quad c p_{1}^{\prime}=c p_{2}^{\prime}=\beta_{0} E_{2}^{\prime}=4 \sqrt{ } 6 / 5 \\
& E_{1}^{\prime}=e_{0}-E_{2}^{\prime}=11 / 5
\end{aligned}
$$

The origin $0_{1}$ of the vector $\mathrm{CP}_{1}$ is within the ellipsoid since $e_{1}<e_{2}, P_{1}<1$. 
28. Fusion (TAR, I=1). For an arbitrarily given colliding system $A\left(e_{h}\right)$, of class $\left\{C P_{0}, E_{0}\right\}$, with critical energy $e_{0}=\left(E_{0}^{2}-c p_{0}^{2}\right)^{\frac{1}{2}}>e_{a}$, a fusion $A\left(e_{h}\right) \rightarrow S(e)$ is always a mathematical possibility, provided only that the single particle resulting has a rest energy e precisely equal to $e_{0}$. While the reverse of a decay process, there is here the physical implication that the fused particle incorporate into its rest energy the given critical energy of the system to which $A$ belongs.

In a collision with target at rest, we shall therefore consider the fusion

$$
A\left(e_{1}, e_{2}\right) \rightarrow S\left(e_{0}\right)
$$

where by definition

$$
e_{0}=\left(e_{a}^{2}+2 e_{2} k_{1}\right)^{\frac{3}{2}}\left(>e_{a}\right)
$$

Technically, the Q-value of $(F)$ is then the negative number

$$
Q=e_{a}-e_{0}<0
$$

and

$$
k_{T}=\frac{e_{a}+e_{o}}{2 e_{2}}(-Q)=\frac{e_{o}^{2}-e_{a}^{2}}{2 e_{2}}=k_{1}
$$

Thus a fusion always occurs exactly "on threshold." (Theorem 27.1.)

The fused particle rides at the $\mathrm{CM}$ of $\mathrm{A}$, with the class velocity $V_{0}$ and energy $E_{0}$, indeed with all its parameters those of the class $\left\{C P_{0}, E_{0}\right\}$ of $\mathrm{A}$. 
The energy conservation equation

$$
k_{1}+Q=k_{0}\left(<k_{1}\right)
$$

indicates a conversion of kinetic to rest energy. (We recall that a coherent system has the least k.e. and greatest proper energy of all systems of its class (Corollary 15.2).

In the $\Sigma^{\prime}$ frame of the class, the two particles of $A^{\prime}$ fuse into a motionless one, with a total conversion of kinetic to rest energy:

$$
k_{a}^{\prime}+Q=k_{0}^{\prime}=0, \quad k_{a}^{\prime}=(-Q)
$$

A fusion sometimes results in a particle which possesses a (more or less) stable ground state of rest energy $e_{g}$. In such a case, one has necessarily

$$
e_{o} \geq e_{g}
$$

and the fused particle is said to be formed with an "energy of excitation"

$$
e^{*}=e_{0}-e_{g} \geq 0 .
$$

If the fusion occurs at vanishingly small incident energies $k_{1}$, as it does for the neutron capture

$$
n^{\circ}+\left({ }^{235} U\right)+\left({ }^{236} U\right) *
$$


then, since $e_{0}=\left(e_{a}^{2}+2 e_{2} k_{1}\right)^{\frac{1}{2}} \rightarrow e_{a}$ as $k_{1}+0$, necessarily $e_{a} \geq e_{g}$ and the intrinsic energy

$$
e_{m}^{*}=e_{a}-e_{g}
$$

is the "minimum energy of excitation" with which the particle can be formed (Fig. 1). In the case cited, one finds from Table IV that

$$
e_{m}^{\star}=e\left({ }^{235} U\right)+e\left(n^{0}\right)-e\left({ }^{236} U\right)=6.4 \mathrm{MeV}
$$

This is more than $\left({ }^{236} \mathrm{U}\right)$ can stand and results in fission $85 \%$ of the time. In "radiative capture" (neutron capture followed by $\gamma$ emission, a less drastic result) the excited nucleus formed in the fusion

$$
n^{0}+\left(\begin{array}{c}
A \\
Z
\end{array}\right)+\left(\begin{array}{c}
A+1 \\
Z
\end{array}\right) *
$$

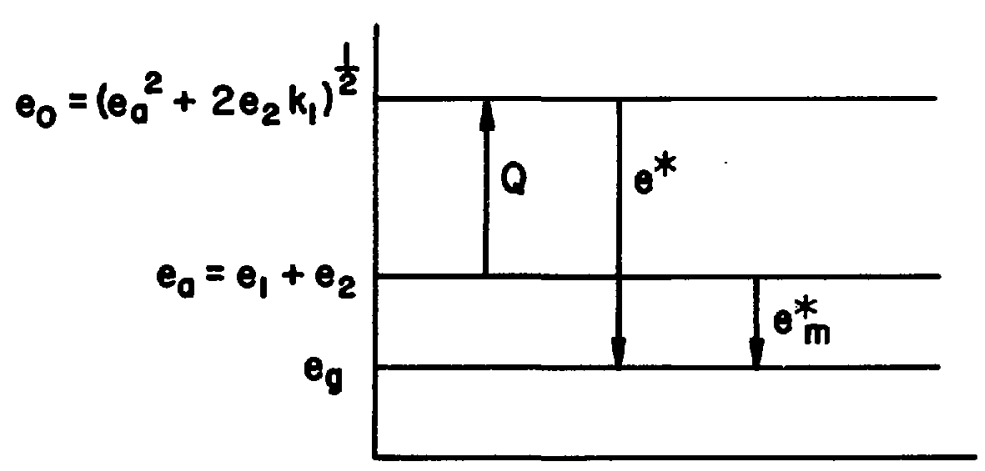

Fig. 1.

$$
A\left(e_{1}, e_{2}\right) \rightarrow S\left(e_{0}\right)
$$

has a ground state to which it may drop by emitting a photon: 


$$
\left(\begin{array}{c}
A+1 \\
Z
\end{array}\right) *\left(\begin{array}{c}
A+1 \\
Z
\end{array}\right)+\gamma
$$

The Q-value of the latter "decay" is then precisely the energy of excitation with which $\left(\begin{array}{c}A+1 \\ Z\end{array}\right)$ was formed in the fusion (12).

Nuclear $\gamma$ absorption

$$
\gamma+\left(\frac{A}{Z}\right) \rightarrow\left(A_{Z}^{A}\right) *
$$

provides a further example. Here the ground state rest energy is $e_{2}$ itself, and the energy of excitation takes the simple form

$$
e^{\star}=e_{o}-e_{g}=\left(e_{2}^{2}+2 e_{2} k_{1}\right)^{\frac{1}{2}}-e_{2}=e_{2}\left\{-1+\left(1+\frac{2 k_{1}}{e_{2}}\right)^{\frac{1}{2}}\right\}
$$

Example 1. If the collision of Example 27.1 results in fusion, the product has rest mass $e_{0}=5$, energy $E_{0}=7$, and momentum $\mathrm{CP}_{0}=(2 \sqrt{6}, 0,0)$. The $Q$-value of the reaction is $Q=e_{a}-e_{0}=-2$, and 2 units of k.e. are converted to rest energy:

$$
\begin{aligned}
& k_{a}+e_{a}=k_{0}+e_{0} \\
& 4+3=2+5
\end{aligned}
$$

29. Two particle product systems (TAR, $I=2)$. We have given the class parameters for a "target at rest" system $A\left(e_{1}, e_{2}\right)$ in 527 , and discussed in $\$ 28$ the case of fusion, where the resulting system consists of $I=1$ particle. We now consider, in a general way, transmutations of form 


$$
A\left(e_{1}, e_{2}\right) \rightarrow S\left(e_{3}, e_{4}\right)
$$

with $I=2$ non-coherent product particles, and will focus attention on $e_{3}$. Since $I=2$, we have uniquely

$$
E_{3}^{\prime}=\left(e_{0}^{2}+e_{3}^{2}-e_{4}^{2}\right) / 2 e_{0}, \quad k_{3}^{\prime}=\frac{k_{s}^{\prime}}{e_{0}}\left(e_{4}+\frac{1}{2} k_{s}^{\prime}\right)
$$

for the energies of $e_{3}$ in the $\Sigma$ ' frame of the class, where

$$
e_{0}=\left(e_{a}^{2}+2 e_{2} k_{1}\right)^{\frac{1}{2}}>e_{s}, \quad k_{s}^{\prime}=e_{0}-e_{s}=k_{a}^{\prime}+Q>0
$$

are the critical energy of the class, and the total $\Sigma^{\prime}$ k.e. of $S^{\prime}$.

Since the class $\left\{\mathrm{CP}_{0}, \mathrm{E}_{0}\right\}$, with its parameters $\gamma_{0}, \beta_{0}, \Psi_{0}=\Psi_{1}$ are given, and $E_{3}^{\prime}, C p_{3}^{\prime}=\left(E_{3}^{\prime 2}-e_{3}^{2}\right)^{\frac{1}{2}}$ are fixed, the equations of 513 are relevant, and we have for standard axes the following summary.

$$
\begin{aligned}
& c p_{3} / c p_{3}^{\prime}=\gamma_{0} D^{\prime}, \quad D^{\prime}=\left\{\left(a_{3 x}^{\prime}+\rho_{3}^{\prime}\right)^{2}+\gamma_{0}^{-2}\left(1-a_{3 x}^{\prime 2}\right)\right\}^{\frac{1}{2}} \\
& \rho_{3}^{\prime} \equiv \beta_{0} E_{3}^{\prime} / c p_{3}^{\prime}=\beta_{0} / \beta_{3}^{\prime} \\
& a_{3 x}=\gamma_{0}\left(a_{3 x}^{\prime}+\rho_{3}^{\prime}\right) / \gamma_{0} D^{\prime} \\
& c p_{3} / c p_{3}^{\prime}=\rho_{3}^{\prime}\left\{a_{3 x} \pm\left(a_{3 x}^{2}+K\right)^{\frac{1}{2}}\right\} / \gamma_{0}\left(1-\beta_{0}^{2} a_{3 x}^{2}\right) \\
& K \equiv \gamma_{0}^{2}\left(1-\beta_{0}^{2} a_{3 x}^{2}\right)\left(\frac{1}{\left.\rho_{3}^{\prime 2}-1\right)}\right. \\
& a_{3 x}^{\prime}=\rho_{3}^{\prime}\left\{-1+\frac{a_{3 x}\left[a_{3 x} \pm\left(a_{3 x}^{2}+K\right)^{\frac{1}{2}}\right]}{\gamma_{0}^{2}\left(1-\beta_{0}^{2} a_{3 x}^{2}\right]}\right\}
\end{aligned}
$$


The usual three cases for $e_{3}$ are distinguished by the inequalities $\rho_{3}^{\prime} \equiv \beta_{0} E_{3}^{\prime} / C p_{3}^{\prime} \lesseqgtr 1$ as indicated in 513. For completeness, we list the main results.

$$
\begin{aligned}
& \text { Case 1. } \quad \rho_{3}^{\prime}<1 . \quad(+) \text { in }(6),(8),-1 \leq a_{3 x} \leq 1 \\
& \text { For } e_{3}=0, \rho_{3}^{\prime}=\beta_{0},(3)-(8) \text { become } \\
& c p_{3} / c p_{3}^{\prime}=\gamma_{0}^{d^{\prime}}, \quad d^{\prime}=1+\beta_{0} a_{3 x}^{\prime} \\
& a_{3 x}=\left(a_{3 x}^{\prime}+\beta_{0}\right) / d^{\prime} \\
& c p_{3} / c p_{3}^{\prime}=1 / \gamma_{0}\left(1-\beta_{0} a_{3 x}\right) \\
& a_{3 x}^{\prime}=\left(a_{3 x}-\beta_{0}\right) /\left(1-\beta_{0} a_{3 x}\right)
\end{aligned}
$$

Case II. $\rho_{3}^{\prime}=1 . \quad(+)$ in $(6),(8), 0 \leq a_{3 x} \leq 1$.

The formulas become those of 13.12 , with the obvious insertion of index 3 . This case is not a mathematical technicality. It occurs in elastic scattering when $e_{1}=e_{2}$.

Case III. $\rho_{3}^{\prime}>1 . \quad\left( \pm\right.$ in (6), (8). $\hat{a}_{3 x} \leq a_{3 x} \leq 1$

$$
\hat{a}_{3 x}=\left(\frac{\rho_{3}^{\prime 2}-1}{\rho_{3}^{\prime 2}-\beta_{0}^{2}}\right)^{\frac{1}{2}}
$$

Further details will be found in $\$ 13$.

Analysis of case dependence. We give below the dependence of cases (for $e_{3}>0$ ) on the relative sizes of $k_{1}$ and the proper energies involved in the transmutation.

It is easy to verify the equivalence of the following inequalities: 
$\rho_{3}^{\prime} \lesseqgtr 1 ; \beta_{0} E_{3}^{\prime} \lesseqgtr c p_{3}^{\prime}=\left(E_{3}^{\prime 2}-e_{3}^{2}\right)^{\frac{1}{2}} ; e_{3} \gamma_{0} \lesseqgtr E_{3}^{\prime} ; e_{3} E_{0} \lesseqgtr e_{0} E_{3}^{\prime} ; 2 e_{3}\left(k_{1}+e_{1}+e_{2}\right) \lesseqgtr$

$e_{0}^{2}+e_{3}^{2}-e_{4}^{2}=e_{1}^{2}+2 e_{1} e_{2}+e_{2}^{2}+2 e_{2} k_{1}+e_{3}^{2}-e_{4}^{2} ; e_{4}^{2}-\left(e_{1}+e_{2}-e_{3}\right)^{2} \lesseqgtr$

$2\left(e_{2}-e_{3}\right) k_{1} ;\left\{e_{4}+\left(e_{1}+e_{2}-e_{3}\right)\right\}\left\{e_{4}-\left(e_{1}+e_{2}-e_{3}\right)\right\} \lesseqgtr 2\left(e_{2}-e_{3}\right) k_{1} ;\left(2 e_{4}+Q\right)(-Q) \leqq$

$2\left(e_{2}-e_{3}\right) k_{1}$. It follows that cases $I, I I$, and III for $e_{3}$ obtain according as

$$
\left(e_{4}+\frac{1}{2} Q\right)(-Q) \stackrel{<}{>}\left(e_{2}-e_{3}\right) k_{1}
$$

This inequality conceals a plethora of special cases, and we shall restrict the present dicussion to collisions in which

$$
e_{4}+\frac{1}{2} Q \equiv \frac{1}{2}\left(e_{1}+e_{2}+e_{4}-e_{3}\right)>0, \quad e_{2} \geq e_{3}
$$

Even now there remain many possibilities, which we outline because of their physical importance.

1. $e_{3}=e_{2}(>0$ by assumption)
a. $Q>0\left(\mathrm{e}_{1}>\mathrm{e}_{4}\right)$ Case $\mathrm{I}$.
b. $Q=0\left(e_{1}=e_{4}\right)$ Case II.
c. $Q<0\left(e_{1}<e_{4}\right)$ Case III. $\left(k_{1}>k_{T}\right.$ required $)$

2. $e_{3}<e_{2}$
a. Q $>0$ Case $I$.
b. $Q=0$ Case $I$.
c. $Q<0$. Here the criteria (10) may be written as

$$
(0<) k^{*} \equiv\left(e_{4}+\frac{1}{2} Q\right)(-Q) /\left(e_{2}-e_{3}\right) \leqq k_{1}
$$


Using the definitions $Q=e_{a}-e_{s}, e_{a}=e_{1}+e_{2}, e_{s}=e_{3}+e_{4}$, one can show that the threshold energy $k_{T}=\left(e_{a}+e_{s}\right)(-Q) / 2 e_{2}$ satisfies the relation

$$
\mathrm{k}_{\mathrm{T}} \leq \mathrm{k}^{*}
$$

with equality iff $e_{3}=0$. In fact, (13) is equivalent to $0 \leq e_{3}\left(2 e_{1}-Q\right)$, which is true since $e_{3} \geq 0, Q<0$.

Hence for $e_{3}>0$, all three cases may arise:

$$
\text { III. } k_{T} \leq k_{1}<k^{*} \quad \text { II. } k_{3}=k^{*} \quad \text { I. } k^{*}<k_{1}
$$

in the order of increasing $k_{1}$.

In the special and important case $e_{3}=0$, only case $I$ is possible. In fact, we know that whenever $e_{3}=0$, regardless of other considerations, $0_{3}^{\prime}$ is at the left focus of the ellipse and hence necessarily falls under case I.

Note that for an elastic collision with $Q=0, e_{1}=e_{3}, e_{2}=e_{4}$, the basic inequality (10) is equivalent to the condition $e_{1} \leqq e_{2}$, in agreement with \$27.

The following collisions, all but one of the type considered in this section, are historical landmarks in artificial transmutation. The MeV values of $Q$ and $k_{T}$ may be verified from Tables III and IV. 


\begin{tabular}{|c|c|c|c|}
\hline & $\overline{\mathbf{Q}}$ & $\mathrm{k}$ & \\
\hline${ }_{2}^{4} \mathrm{He}+{ }_{7}^{14} \mathrm{~N} \rightarrow{ }_{8}^{17} \mathrm{O}+{ }_{1}^{1} \mathrm{H}$ & -1.19 & 1.53 & Rutherford, 1914 \\
\hline${ }_{2}^{4} \mathrm{He}+{ }_{4}^{9} \mathrm{Be} \rightarrow{ }_{6}^{12} \mathrm{C}+\mathrm{n}^{\mathrm{O}}$ & 5.7 & - & Chadwick, 1932 \\
\hline $\mathrm{n}^{0}+{ }_{7}^{14} \mathrm{~N} \rightarrow{ }_{6}^{14} \mathrm{C}+{ }_{1}^{1} \mathrm{H}$ & .63 & - & Feather, 1932 \\
\hline${ }_{1}^{1} \mathrm{H}+{ }_{3}^{7} \mathrm{Li} \rightarrow 2\left(_{2}^{4} \mathrm{He}\right)$ & 17.3 & - & Cockcroft, Wa1ton, 1932 \\
\hline${ }_{2}^{4} \mathrm{He}+{ }_{13}^{27} \mathrm{Al} \rightarrow{ }_{15}^{30} \mathrm{P}+\mathrm{n}^{\circ}$ & -2.65 & 3.05 & Joliots, 1934 \\
\hline$\gamma+{ }_{I}^{2} H+{ }_{1}^{1} H+n^{\circ}$ & -2.225 & 2.226 & Chadwick, Goldhaber, 1935 \\
\hline $\mathrm{p}^{+}+\mathrm{p}^{+}+3 \mathrm{p}^{+}+\mathrm{p}^{-}$ & -1877 & 5630 & Segré, Chamberlain, 1954 \\
\hline $\bar{v}_{\varepsilon}+p^{+}+n^{0}+\varepsilon^{+}$ & -1.805 & 1.807 & Reines, Cowan, 1956 \\
\hline $\bar{v}_{\mu}+p^{+} \rightarrow n^{0}+\mu^{+}$ & -107 & 113 & (Brookhaven) 1962 \\
\hline
\end{tabular}


Example 1. If the collision of Example 27.1 results in two particles of equal rest mass 2 , the $\Sigma^{\prime}$ parameters of the resuiting system $S^{\prime}\left(e_{3}, e_{4}\right)$ are

$$
e_{i}=2 \quad E_{i}^{\prime}=e_{0} / 2=5 / 2, \quad c p_{i}^{\prime}=3 / 2, \quad \rho_{i}^{\prime}=\beta_{0} E_{i}^{\prime} / c p_{i}^{\prime}=10 \sqrt{ } 6 / 21>1
$$

The $Q-v a l u e$ is $Q=3-4=-1$, and the threshold is

$$
k_{T}=\frac{e_{a}+e_{s}}{2 e_{2}}(-Q)=\frac{7}{4}<k_{1}=4
$$

This inequality is equivalent to $e_{s}=4<e_{0}=5$.

30. Elastic collision (TAR, $I=2$ ). We consider in this section in general, and in the next two with $e_{1}=0$, the important case of elastic collision on target at rest, of the simple form

$$
A\left(e_{1}, e_{2}\right) \rightarrow S\left(e_{3}, e_{4}\right)
$$

where $e_{1}=e_{3} \geq 0, e_{2}=e_{4}>0$, and hence $Q=0, k_{1}=k_{3}+k_{4}$. We recal1 from $\$ 27$ the Lorentz parameters

$$
e_{0}=\left(e_{a}^{2}+2 e_{2} k_{1}\right)^{\frac{1}{2}}, \quad \gamma_{0}=E_{0} / e_{0}, \quad \beta_{0}=c p_{0} / E_{0}
$$

the $\Sigma^{\prime}$ parameters $E_{2}^{\prime}=\gamma_{0} e_{2}, c p_{h}^{\prime}=\gamma_{0} \beta_{0} e_{2}$, and the fact that $e_{2}$ is always on the ellipsoid $E$, whereas $e_{1}$ is inside, on, or outside $E$ according as $e_{1} \lesseqgtr e_{2}$.

In the corresponding collision $A^{\prime} \rightarrow S^{\prime}$ in the $\Sigma^{\prime}$ frame of the class, 
even the individual energies are unchanged:

$$
k_{1}^{\prime}=k_{3}^{\prime}, \quad k_{2}^{\prime}=k_{4}^{\prime}
$$

and indeed, $A^{\prime}$ and $S^{\prime}$ as systems of class $\left\{0, e_{0}\right\}$ wich $e_{1}+e_{2}<e_{0}$ can differ only in direction. From $\$ 27$ we therefore have at once

$$
\begin{aligned}
& E_{1}^{\prime}=e_{0}-e_{2} \cdot \gamma_{0}=E_{3}^{\prime} \quad E_{2}^{\prime}=e_{2} \gamma_{0}=E_{4}^{\prime} \\
& C P_{1}^{\prime}=C P_{3}^{\prime}=\gamma_{0} \beta_{0} e_{2}=c p_{2}^{\prime}=c p_{4}^{\prime}
\end{aligned}
$$

A computational procedure is given in the Example below. To understand the physical nature of such collisions, it is important to study them further as they appear on the standard axes $S, S^{\prime}$. In particular we consider the dependence of the final system on the physically meaningful angle $\psi_{3}^{\prime}\left(0^{\circ} \leq \psi_{3}^{\prime}<180^{\circ}\right)$ at which the projectile scatters from its line of flight in $\Sigma^{\prime}$, namely from the direction $\Psi_{1}^{\prime}=\Psi_{1}=\Psi_{0}$. The limiting cases $\psi_{3}^{\prime}=0^{\circ}$ and $\psi_{3}^{\prime}=180^{\circ}$ will be referred to as a "miss" or a "direct hit" respectively.

$$
\text { Since } c p_{4 x}^{\prime}=-c p_{3 x}^{\prime}=-\gamma_{0} \beta_{0} e_{2} \cos \psi_{3}^{\prime} \text { and } E_{4}^{\prime}=e_{2} \gamma_{0} \text {, we have for the } \Sigma
$$
parameters of the recoiling target

$$
\begin{aligned}
& c p_{4 x}=\gamma_{0}\left(c p_{4 x}^{\prime}+\beta_{0} E_{4}^{\prime}\right)=2 \gamma_{0}^{2} \beta_{0} e_{2} \sin ^{2}\left(\psi_{3}^{\prime} / 2\right) \\
& E_{4}=\gamma_{0}\left(\beta_{0} c p_{4 x}^{\prime}+E_{4}^{\prime}\right)=e_{2}\left\{1+2\left(\gamma_{0}^{2}-1\right) \sin ^{2}\left(\psi_{3}^{\prime} / 2\right)\right\} \\
& k_{4}=E_{4}-e_{2}=2 e_{2}\left(\gamma_{0}^{2}-1\right) \sin ^{2}\left(\psi_{3}^{\prime} / 2\right) \\
& c p_{4}=\left(E_{4}^{2}-e_{2}^{2}\right)^{\frac{1}{2}}
\end{aligned}
$$




$$
a_{4 x}=\cos \psi_{4}=C p_{4 x} / C p_{4}
$$

From the conservation laws we may then obtain for the $\Sigma$ parameters of the scattered projectile:

$$
\begin{aligned}
& c p_{3 x}=c p_{0}-c p_{4 x}=\gamma_{0} B_{0}\left(e_{0}-2 e_{2} \gamma_{0} \sin ^{2}\left(\psi_{3}^{1} / 2\right)\right. \\
& E_{3}=E_{0}-E_{4} \\
& k_{3}=k_{1}-k_{4}\left(=E_{3}-e_{1}\right) \\
& c p_{3}=\left(E_{3}^{2}-e_{1}^{2}\right)^{\frac{1}{2}} \\
& a_{3 x}=\cos \psi_{3}=c p_{3 x} / c p_{3}
\end{aligned}
$$

The following remarks are now in order. (cf. Fig. 1)

(1) The position of $0_{2}^{\prime \prime}$ on the ellipsoid $E$ indicates that Case II always obtains for the target, which therefore scatters forward in $\Sigma$, its angle $\psi_{4}$ ranging from a (1imiting) $90^{\circ}$ to $0^{\circ}$ as the projectile angle $\psi_{3}^{\prime}$ ranges from $0^{\circ}$ (miss) to $180^{\circ}$ (direct hit) in $\Sigma^{\prime}$. The exceptional case $C p_{4}=0 \quad\left(E_{4}=e_{2}, k_{4}=0\right)$ in (8) occurs only in the event of a miss, with the trivial result $A \equiv S$. (Note 1.) 


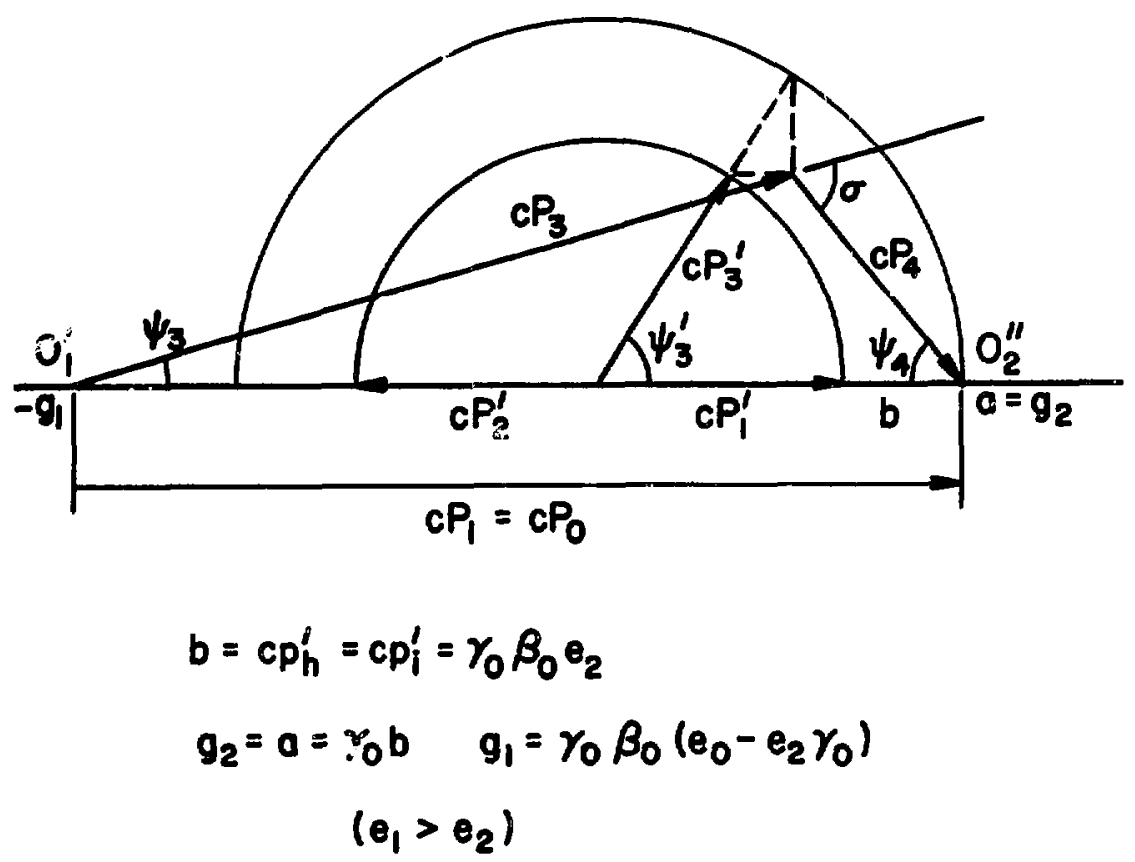

Fig. 1.

\section{Elastic collision}

(2) As we know, the dependence of the projectile angle $\psi_{3}$ on $\psi_{3}^{\prime}$ is more complex, the range of $\psi_{3}$ depending on the three possible cases.

I. $e_{1}<e_{2}\left(O_{1}^{\prime}\right.$ inside E) with $0^{\circ} \leq \psi_{3} \leq 180^{\circ}$.

II. $e_{1}=e_{2}\left(O_{1}^{\prime}\right.$ on E) with $0^{\circ} \leq \psi_{3}<90^{\circ}$, the minimum angle $\theta$ of separation being given by (20.5)

$$
\sin (\hat{\sigma} / 2)=1 /\left(1+\gamma_{0}^{2}\right)^{\frac{1}{2}}
$$


III. $e_{1}>e_{2}\left(O_{1}\right.$ outside $\left.E\right)$ with $0^{\circ} \leq \psi_{3} \leq \hat{\psi}_{3}<90^{\circ}$.

The parameters associated with the tangential angle $\hat{\psi}_{3}$ are given in (13.13), (13.15), (13.16). In the present case, we know

$$
\begin{aligned}
& \rho_{3}^{\prime 2}=\beta_{0}^{2} E_{3}^{\prime 2} / C p_{3}^{\prime 2}=\beta_{0}^{2}\left(e_{0}-e_{2} \gamma_{0}\right)^{2} / \gamma_{0}^{2} \beta_{0}^{2} e_{2}^{2} \text {. Since }\left(e_{0}-e_{2} \gamma_{0}\right)^{2}= \\
& \left(e_{1}^{2}+e_{2}^{2}+2 e_{2} E_{1}\right)-2 e_{2}\left(E_{1}+e_{2}\right)+e_{2}^{2} \gamma_{0}^{2}=e_{1}^{2}-e_{2}^{2}+e_{2}^{2} \gamma_{0}^{2} \text {, we see that } \\
& \gamma_{0}^{2}\left(\rho_{3}^{\prime 2}-1\right)=\left(\frac{e_{1}}{e_{2}}\right)^{2}-1
\end{aligned}
$$

and hence from (13.13)

$$
\hat{a}_{3 x}=\cos \hat{\psi}_{3}=\left\{1-\left(e_{2} / e_{1}\right)^{2}\right\}^{\frac{1}{2}}
$$

The exceptional case $c p_{3}=0$ in (13) $\left(E_{3}=e_{1}, k_{3}=0\right)$ occurs only in the event of a direct hit on a target of equal rest mass $\left(\psi_{3}^{\prime}=180^{\circ}, e_{1}=e_{2}\right)$ under Case II above. From (11) it appears that the target recoils with the whole k.e. $k_{4}=k_{1}$ of the projectile, which is stopped dead in its tracks, just as in classical mechanics. (Note 2.)

(3) The relations (13.5-13.10) apply here (with subscript $i=3,4$ ) to the scattered projectile and recoil target, giving the dependence of $c p_{i}$ and $a_{i x}$ on $a_{i x}^{\prime}$, and of $c p_{i}$ and $a_{i x}^{\prime}$ on $a_{i x}$. It may be noted that, in the present case,

$$
\begin{aligned}
& a_{4 x}^{\prime}=-a_{3 x}^{\prime}=-\cos \psi_{3}^{\prime} \\
& \rho_{4}^{\prime}=1 \quad \text { Case II. See }(13.12) \\
& \rho_{3}^{\prime}=\left(e_{0}-e_{2} \gamma_{0}\right) / \gamma_{0} e_{2} \leqq 1 \text { as } e_{1} \leqq e_{2} \cdot \text { Cases I, II, III. }
\end{aligned}
$$


(4) We also recall the simple relations (13.27)

$$
a_{i x}=\left(E_{i}-\gamma_{0}^{-1} E_{i}^{\prime}\right) / \beta_{0} c p_{i} ; \quad i=3,4
$$

expressing $a_{i x}$ as a single valued function of $c p_{i}$ and $E_{i}=\left(c p_{i}^{2}+e_{i}^{2}\right)^{\frac{1}{2}}$.

Substituting the present values

$$
E_{3}^{\prime}=e_{0}-\gamma_{0} e_{2} \quad E_{4}^{\prime}=\gamma_{0} e_{2}
$$

and using the relations

$$
\gamma_{0}=E_{0} / e_{0}, \quad B_{0}=c p_{1} / E_{0}, \quad E_{0}=E_{1}+e_{2}, \quad e_{0}^{2}=e_{1}^{2}+e_{2}^{2}+2 e_{2} E_{1}
$$

one may obtain from (17) the evaluations

$$
\begin{aligned}
a_{3 x} & =E_{0}\left(E_{3}-\gamma_{0}^{-1}\left(e_{0}-\gamma_{0} e_{2}\right)\right) / c p_{1} c p_{3} \\
& =\left(E_{0} E_{3}-e_{0}^{2}+E_{0} e_{2}\right) / c p_{1} c p_{3} \\
& =\left(E_{0} E_{3}-\left(e_{1}^{2}+e_{2}^{2}+2 e_{2} E_{1}\right)+\left(\left(E_{1}+e_{2}\right) e_{2}\right) / c p_{1} c p_{3}\right. \\
& =\left(E_{0} E_{3}-e_{1}^{2}-e_{2} E_{1}\right) / c p_{1} c p_{3}
\end{aligned}
$$

where

$$
\begin{aligned}
E_{3} & =\left(c p_{3}^{2}+e_{1}^{2}\right)^{\frac{1}{2}} \\
a_{4 x} & =E_{0}\left(E_{4}-\gamma_{0}^{-1}\left(\gamma_{0} e_{2}\right) / c p_{1} c p_{4}\right. \\
& =E_{0}\left(E_{4}-e_{2}\right) / c p_{1} c p_{4}=E_{0} k_{4} / c p_{1} c p_{4}
\end{aligned}
$$


where

$$
E_{4}=\left(c p_{4}^{2}-e_{2}^{2}\right)^{\frac{1}{2}}
$$

Note 1. If $c p_{4}=0$ under remark (1) above, then $E_{4}=e_{2}$ by (7), $k_{4}=0$, $\psi_{3}^{:}=0^{\circ}$ (miss) by (6), and $k_{3}=k_{1}$ by (11).

Note 2. If $\mathrm{Cp}_{3}=0$ under remark (2) above, we have at once $\mathrm{E}_{3}=\mathrm{e}_{1}$ in (12), so $k_{3}=0$, and $k_{1}=k_{4}$ in (11). Moreover, by (6),

$$
\begin{aligned}
k_{1} & =2 e_{2}\left(\gamma_{0}^{2}-1\right) \sin ^{2} \psi_{3}^{1 / 2} \\
& =\left(2 e_{2} / e_{0}^{2}\right)\left(E_{0}^{2}-e_{0}^{2}\right) \sin ^{2} \psi_{3}^{\prime / 2} \\
& =\left(2 e_{2} / e_{0}^{2}\right)\left(\left(E_{1}^{2}+2 e_{2} E_{1}+e_{2}^{2}\right)-\left(e_{1}^{2}+e_{2}^{2}+2 e_{2} E_{1}\right)\right) \sin ^{2} \psi_{3}^{\prime / 2} \\
& =\left(2 e_{2} / e_{0}^{2}\right)\left(E_{1}^{2}-e_{1}^{2}\right) \sin ^{2} \psi_{3}^{1 / 2} \\
& =\left(2 e_{2} / e_{0}^{2}\right)\left(E_{1}+e_{1}\right)\left(k_{1}\right) \sin ^{2} \psi_{3}^{\prime} / 2 . \text { Hence } \\
e_{0}^{2} & \equiv e_{1}^{2}+e_{2}^{2}+2 e_{2} E_{1}=2 e_{2}\left(E_{1}+e_{1}\right) \sin ^{2} \psi_{3}^{\prime} / 2 \leq 2 e_{2} E_{1}+2 e_{2} e_{1}
\end{aligned}
$$

and

$$
\left(e_{1}-e_{2}\right)^{2} \leq 0 \text {. Hence } e_{1}=e_{2} \text {, and } \sin ^{2} \psi_{3}^{\prime} / 2=1, \psi_{3}^{\prime}=180^{\circ}
$$

(direct hit).

Example 1. A particle of rest energy 5, k.e. 8, direction $(2 / 3,2 / 3,1 / 3)_{A}$ collides elastically with a particle of rest energy 7 , at rest in $\Sigma$. The auxiliary direction $\Omega^{\prime}=(2 / 7,6 / 7,3 / 7)$ is used (in both methods below) to locate the direction of scattering of the projectile about its $\Sigma$ ' line of flight $\Psi_{1}$. We require the final projectile parameters on $\Sigma$ axes $A$. From $A\left(e_{1}, e_{2}\right)$ we find 


$$
\begin{array}{llll}
e_{1}=5 & E_{1}=13 & c P_{1}=12 & c P_{1}=(8,8,4)_{A} \\
e_{2}=7 & E_{2}=7 & c P_{2}=0 & c P_{2}=(0,0,0)_{A} \\
e_{a}=12 & E_{0}=20 & c P_{0}=12 & c P_{0}=(3,8,4)_{A} \\
e_{0}=16 & \gamma_{0}=5 / 4 & B_{0}=3 / 5 & \Psi_{0}=(2 / 3,2 / 3,1 / 3)_{A}
\end{array}
$$

For systems $A^{\prime}\left(e_{1}, e_{2}\right), S^{\prime}\left(e_{3}, e_{4}\right)$ in $\Sigma^{\prime}$,

$$
\begin{aligned}
& E_{4}^{\prime}=E_{2}^{\prime}=\gamma_{0} e_{2}=35 / 4 \quad E_{3}^{\prime}=E_{+}^{\prime}=e_{0}-E_{2}^{\prime}=29 / 4 \\
& c P_{h}^{\prime}=\gamma_{0} B_{0} c_{2}=21 / 4=c p_{i}^{\prime}
\end{aligned}
$$

There are now two alternative methods (Note 22.1)

I. Using the parallel axes $A, A^{\prime}$ of (10.5), we find from Appendix $I$, Corollary 1 a rotation $\delta$ taking $X^{\prime}$ into $\Psi_{1}^{\prime}=\Psi_{0}=(2 / 3,2 / 3,1 / 3)_{A}$, with matrix

$$
D=\left[\begin{array}{lll}
2 / 3 & -2 / 3 & -1 / 3 \\
2 / 3 & 11 / 15 & -2 / 15 \\
1 / 3 & -2 / 15 & 14 / 15
\end{array}\right]
$$

We then take for the $\Sigma^{\prime}$ direction of the scattered projectile

$$
\left(\Psi_{3}^{\prime}\right)_{A^{\prime}}=D\left(\Omega^{\prime}\right)_{A^{\prime}}=(-11,16,8) / 21
$$

so that

$$
E_{3}^{\prime}=29 / 4 \text { and }\left(\mathrm{CP}_{3}^{\prime}\right)_{A^{\prime}}=(21 / 4)\left(\Psi_{3}^{\prime}\right)_{A^{\prime}}=(-11 / 4,4,2)_{A^{\prime}}
$$




$$
\begin{aligned}
& \left(c P_{3}\right)_{A}=\left[\begin{array}{c}
-11 / 4 \\
4 \\
2
\end{array}\right]+\{(1 / 4)(3 / 2)+(3 / 4)(29 / 4)\}\left[\begin{array}{l}
2 / 3 \\
2 / 3 \\
1 / 3
\end{array}\right]=\left[\begin{array}{l}
9 / 8 \\
63 / 8 \\
63 / 16
\end{array}\right] \\
& E_{3}=5 / 4\{(3 / 5)(3 / 2)+(29 / 4)\}=163 / 16
\end{aligned}
$$

Ck. $\quad E_{3}^{2}-c p_{3}^{2}=e_{1}^{2}$

II. One may prefer to use standard axes $S, S^{\prime}$. We first define

$$
\left(\Psi_{3}^{\prime}\right)_{S^{\prime}}=\left(\Omega^{\prime}\right)_{S^{\prime}}=(2 / 7,6 / 7,3 / 7)_{S^{\prime}}
$$

so

$$
\left(C P_{3}^{\prime}\right)_{A^{\prime}}=(21 / 4)\left(\Psi_{3}^{\prime}\right)_{S^{\prime}}=(3 / 2,9 / 2,9 / 4)_{S^{\prime}}
$$

Then (10.2) gives $\left(\mathrm{CP}_{3}\right)_{S}$ as

$$
\begin{aligned}
& \overline{c p}_{3 x}=5 / 4\left\{(3 / 2+(3 / 5)(29 / 4)\}=117 / 16, \overline{c p}_{3 y}=9 / 2, \overline{c p}_{3 z}=9 / 4,\right. \text { and } \\
& \left.E_{3}=5 / 4((3 / 5)(3 / 2)+29 / 4)\right)=163 / 16
\end{aligned}
$$

The rotation $\delta$ taking $X$ into $\Psi_{0}$ has the matrix $D$ of method $I$ (although used there for a different purpose) so we obtain

$$
\left(\mathrm{cP}_{3}\right)_{A}=\mathrm{D}\left(\mathrm{cP}_{3}\right)_{S}=(9 / 8,63 / 8,63 / 16)_{A}
$$


Note 3. Elastic collision (TAR, $I=2, e_{1}=e_{2}>0$ ). We set up some of the basic relations involved in Moller (electron-electron) scattering, in which the fundamental parameters are $e, k_{1}, \gamma_{1}$.

For the incident system $A\left(e_{1}, e_{2}\right)$, we have

$$
\begin{aligned}
& e_{1}=\mathrm{e}>0 \quad \mathrm{k}_{1}>0 \quad \gamma_{1}=1+k_{1} / \mathrm{e} \quad \mathrm{E}_{1}=\gamma_{1} \mathrm{e} \quad c p_{1}=\mathrm{e}\left(\gamma_{1}^{2}-1\right)^{\frac{1}{2}} \quad \mathrm{cP} \mathrm{P}_{1}=c p_{1} \Psi_{1} \\
& e_{2}=\mathrm{e} \quad \mathrm{k}_{2}=0 \quad \gamma_{2}=1 \quad E_{2}=\mathrm{e} \quad \mathrm{c} p_{2}=0 \quad \mathrm{cP} P_{2}=0
\end{aligned}
$$

The class of $A\left(e_{1}, e_{2}\right)$ therefore has parameters

$$
\begin{aligned}
& E_{0}=e\left(\gamma_{1}+1\right) \quad c P_{0}=c P_{1} \quad c p_{0}=e\left(\gamma_{1}^{2}-1\right)^{\frac{1}{2}} \quad e_{0}=e\left\{\left(2\left(\gamma_{1}+1\right)\right\}^{\frac{1}{2}}\right. \\
& \gamma_{0}=E_{0} / e_{0}=\left\{\left(\gamma_{1}+1\right) / 2\right\}^{\frac{1}{2}} \quad B_{0}=\left\{\left(\gamma_{0}-1\right) /\left(\gamma_{0}+1\right)\right\}^{\frac{1}{2}} \quad \psi_{0}=\Psi_{1} \cdot
\end{aligned}
$$

In the $\Sigma^{\prime}$ frame of the class, system $A^{\prime}\left(e_{1}, e_{2}\right)$ has

$$
E_{1}^{\prime}=E_{2}^{\prime}=e \gamma_{0}=e_{0} / 2=E_{3}^{\prime}=E_{4}^{\prime}, \quad c p_{h}^{\prime}=\gamma_{0} \beta_{0} e=c p_{i}^{\prime},
$$

both origins $O_{1}^{\prime}, O_{2}^{\prime \prime}$ being on the momentum ellipsoid (Case $I I, \rho_{h}^{\prime}=1=\rho_{i}^{\prime}$ ). Moreover, from the energy values it appears that

$$
\gamma_{h}^{\prime}=\gamma_{0}=\gamma_{i}^{\prime}
$$

In particular, one sees that

$$
\gamma_{1}^{\prime}=\gamma_{0}=\left\{\left(\gamma_{1}+1\right) / 2\right\}^{\frac{1}{2}}
$$


From the Lorent $z$ transformation on standard axes one obtains the $\Sigma$ parameters of the scattered projectile as functions of its $\Sigma$ ' scattering

$$
\begin{aligned}
& \cos \psi_{3}^{\prime} \equiv a_{3 x}^{\prime} \equiv a^{\prime} \\
& c p_{3 x}=\gamma_{0}\left(c p_{3}^{\prime} a^{\prime}+\beta_{0} E_{3}^{\prime}\right)=\gamma_{0}\left(\gamma_{0} \beta_{0} e a^{\prime}+\beta_{0} \gamma_{0} e\right)=\gamma_{0}^{2} \beta_{0} e\left(1+a^{\prime}\right) \\
& E_{3}=\gamma_{0}\left(\beta_{0} c p_{3}^{\prime} a^{\prime}+E_{3}^{\prime}\right)=\gamma_{0}\left(\beta_{0}^{2} \gamma_{0} e a^{\prime}+\gamma_{0} e\right)=e\left(\gamma_{0}^{2}+\left(\gamma_{0}^{2}-1\right) a^{\prime}\right) \\
& =e\left(1+\left(\gamma_{0}^{2}-1\right)\left(1+a^{\prime}\right)\right)
\end{aligned}
$$

Hence the scattered projectile has k.e. $k_{3}=e\left(\gamma_{0}^{2}-1\right)\left(1+a^{\prime}\right)$, or by (24),

$$
k_{3}=e\left(\gamma_{1}-1\right) \cdot \frac{1}{2}\left(1+a^{\prime}\right)=k_{1} \cdot \frac{1}{2}\left(1+a^{\prime}\right)
$$

Thus the recoil target has k.e.

$$
k_{4}=k_{1}-k_{3}=k_{1} \cdot 1_{2}\left(1-a^{\prime}\right)
$$

(In Moller scattering, the $\Sigma$ ' cross section is given as a function of the variable $W \equiv\left(1-a^{\prime}\right) / 2$, in terms of which $k_{3}=k_{1}(1-W), k_{4}=k_{1} W$. We do not include here a discussion of this cross section, nor methods of sampling it.)

Since $\rho_{3}^{\prime}=1$ (Case II) the direction transformation from $a^{\prime} \equiv a_{3 x}^{\prime}=\cos \psi_{3}^{\prime}$ to $a \equiv a_{3 x}=\cos \psi_{3}$ of $(9.2)$ reads

$$
a=\left(a^{\prime}+1\right) /\left\{\left(a^{\prime}+1\right)^{2}+\gamma_{0}^{-2}\left(1-a^{\prime 2}\right)\right\}^{\frac{1}{2}}
$$

and may be used to compute the $\Sigma$ deflection from a selected $\Sigma^{\prime}$ deflection. 
As an alternative, we may eliminate $a^{\prime}$ from (25) and (26) to obtain $E_{3}-B_{0} c P_{3 x}=\gamma_{0} \cdot \gamma_{0}^{-2} E_{3}^{\prime}=E_{3}^{\prime} / \gamma_{3}^{\prime}=e$, so that $c p_{3 x}=k_{3} / \beta_{0}$ and hence

$$
a=c p_{3 x} / c p_{3}=k_{3} / \beta_{o} c p_{3}
$$

where $c p_{3}=\left(E_{3}^{2}-e^{2}\right)^{\frac{1}{2}}=\left\{k_{3}\left(k_{3}+2 e\right)\right\}^{\frac{1}{2}}$, and $k_{3}$ is obtained from (27).

In Moller scattering it is important to invert (29), with the resuit $a^{\prime}=\left(a^{2}-\gamma_{0}^{2}\left(1-a^{2}\right)\right) /\left(a^{2}+\gamma_{0}^{2}\left(1-a^{2}\right)\right)$. Using (21), this gives

$$
a^{\prime}=\left(2-\left(\gamma_{1}+3\right)\left(1-a^{2}\right)\right) /\left(2+\left(\gamma_{1}-1\right)\left(1-a^{2}\right)\right)
$$

(Setting $W=\frac{1}{2}\left(1-a^{\prime}\right)$, this becomes

$$
w=\left(\gamma_{1}+1\right)\left(1-\mathrm{a}^{2}\right) /\left(2+\left(\gamma_{1}-1\right)\left(1-\mathrm{a}^{2}\right)\right)
$$

which is used to determine the range of $W$ from the range of a.)

31. Elastic collision ( $T A R, I=2, e_{1}=0$, Compton) It was first noted by Compton and Debye that the laws of elastic coliision govern the scattering of x-rays by free electrons, provided the "rays" are regarded as particles of mass $M=h \nu / c^{2}$ and absolute momentum $M c=h v / c$.

For the present section we define "Compton scattering" as any elastic collision of form

$$
A\left(e_{1}, e_{2}\right) \rightarrow S\left(e_{3}, e_{4}\right)
$$

where $e_{1}=e_{3}=0$ and $e_{2}=e_{4}>0$, in which an immaterial particle scatters from a material target, and consider under the TAR assumption an initiai system $A\left(0, e_{2}\right)$ with parameters 


$$
\begin{array}{lllll}
e_{1}=0 & k_{1}>0 & E_{1}=k_{1} & c P_{1}=k_{1} & c P_{1}=k_{1} \Psi_{1} \\
e_{2}>0 & k_{2}=0 & E_{2}=e_{2} & c p_{2}=0 & c P_{2}=0 \\
e_{a}=e_{2} & k_{a}=k_{1} & E_{0}=k_{1}+e_{2} & c P_{0}=k_{1} & c P_{0}=c P_{1}
\end{array}
$$

Note that the basic scalars are $e_{2}$ and $k_{1}$. If $e_{2}$ is taken as the unit of energy, as is customary, all formulas may be read with $e_{2}=1$. The Lorentz parameters are now

$$
e_{0}=\left(e_{2}^{2}+2 e_{2} k_{1}\right)^{\frac{1}{2}} \quad \gamma_{0}=\left(k_{1}+e_{2}\right) / e_{0} \quad \beta_{0}=k_{1} /\left(k_{1}+e_{2}\right)
$$

as seen from $(30.1)$. Further specialization of 530 to the case $e_{1}=0$ gives the following results.

$O_{2}$ is on the ellipsoid $E$ (Case II) while $O_{1}$ is inside, at its left focus (Case I). The parameters of $A^{\prime}$ and $S^{\prime}$ are

$$
\begin{aligned}
& E_{1}^{\prime}=\gamma_{0} \beta_{0} e_{2}=E_{3}^{\prime} \quad E_{2}^{\prime}=\gamma_{0} e_{2}=E_{4}^{\prime} \\
& c p_{1}^{\prime}=c p_{3}^{\prime}=\gamma_{0} \beta_{0} e_{2}=c p_{2}^{\prime}=c p_{4}^{\prime}
\end{aligned}
$$

(Since $e_{1}=0$, it is obvious that $E_{1}^{\prime}=c p_{1}^{\prime}$, which we know to be $\gamma_{0} \beta_{0} e_{2}$ from 530 . However, formally,

$$
\begin{aligned}
E_{1}^{\prime} & =e_{0}-\gamma_{0} e_{2}=e_{0}-\frac{E_{0}}{e_{0}} e_{2}=\left\{\left(e_{2}^{2}+2 e_{2} k_{1}\right)-\left(k_{1}+e_{2}\right) e_{2}\right\} / e_{0} \\
& \left.=e_{2} k_{1} / e_{0}=e_{2} c p_{0} / e_{0}=e_{2} \gamma_{0} \beta_{0}\right)
\end{aligned}
$$


The $\Sigma$ parameters of $S$ are given by $(30.4)-(30.13)$ with the $\gamma_{0}, B_{0}$ values in (1). In particular, since $\gamma_{0}^{2}-1=\gamma_{0}^{2} \beta_{0}^{2}$, we see from (30.6) that the recoil k.e. of the target is

$$
k_{4}=2 e_{2}\left(k_{1} / e_{o}\right)^{2} \sin ^{2}\left(\psi_{3}^{\prime} / 2\right)
$$

with a range

$$
0 \leq k_{4} \leq 2 e_{2}\left(k_{1} / e_{0}\right)^{2}=k_{1} /\left\{1+\left(e_{2} / 2 k_{1}\right)\right\}
$$

The energy of the scattered projectile is therefore

$$
k_{3}=k_{1}-k_{4}
$$

with a corresponding range

$$
k_{1} \geq k_{3} \geq k_{1} /\left\{1+\left(2 k_{1} / e_{2}\right)\right\}
$$

The formulas (13.11) with subscript $i=3$ govern the parameters of the scattered projectile, while (13.12) with subscript $i=4$ governs those of the recoil target. We need not repeat them all here. We give only the dependence of $a_{3 x}$ and $a_{4 x}$ on $a_{3 x}^{\prime}:$

$$
\begin{aligned}
& a_{3 x}=\left(a_{3 x}^{\prime}+\beta_{0}\right) / d^{\prime}, \quad d^{\prime}=1+\beta_{0} a_{3 x}^{\prime} \\
& a_{4 x}=\left\{\left(1-a_{3 x}^{\prime}\right) /\left[2-\beta_{0}^{2}\left(1+a_{3 x}^{\prime}\right)\right]\right\}^{\frac{1}{2}}
\end{aligned}
$$

Note the ranges $1 \geq a_{3 x} \geq-1$ and $0 \leq a_{4 x} \leq 1$ 
Finally we obtain from $(30.18),(30.19)$

$$
\begin{aligned}
a_{3 x} & =1+\left(e_{2} / k_{1}\right)-\left(e_{2} / k_{3}\right)=\beta_{0}^{-1}-\left(e_{2} / k_{j}\right) \\
a_{4 x} & =k_{4}\left\{1+\left(e_{2} / k_{1}\right)\right\} / c p_{4}= \\
& =\left\{1+\left(e_{2} / k_{1}\right)\right\} /\left\{1+\left(2 e_{2} / k_{4}\right)\right\}^{\frac{1}{2}}=1 / \beta_{0}\left\{1+\left(2 e_{2} / k_{4}\right)\right\}^{\frac{1}{2}}
\end{aligned}
$$

where

$$
k_{1} \geq k_{3} \geq k_{1} /\left\{1+\left(2 k_{1} / e_{2}\right)\right\} \text { and } k_{4}=k_{1}-k_{3} \cdot
$$

We may write (8) in either of the two equivalent forms,

$$
\begin{aligned}
& k_{3}=k_{1} /\left\{1+\left(k_{1} / e_{2}\right)\left(1-a_{3 x}\right)\right\} \\
& k_{3}=e_{2} \beta_{o} /\left(1-\beta_{o} a_{3 x}\right)
\end{aligned}
$$

Since $k_{3}=c p_{3}$, and $e_{2} \beta_{0}=\gamma_{0}^{-1}\left(e_{2} \gamma_{0} \beta_{0}\right)=\gamma_{0}^{-1} c p_{3}^{\prime}$, (11) is actually the polar equation (13.25)

$$
c p_{3}=\gamma_{0}^{-1} c p_{3}^{\prime} /\left(1-\beta_{0} \cos \psi_{3}\right)
$$

and we have come full circle.

The $\Sigma$ angles $\psi_{3}, \psi_{4}$ are correlated by the equation

$$
\tan \psi_{4}=\frac{e_{2}}{e_{2}+k_{1}} \cot \left(\psi_{3} / 2\right)
$$

This may be obtained from the self-evident momentum equations 


$$
\begin{aligned}
& \mathrm{cp}_{4} \sin \psi_{4}=c p_{3} \sin \psi_{3} \\
& c p_{4} \cos \psi_{4}=c p_{0}-c p_{3} \cos \psi_{3}
\end{aligned}
$$

Dividing the first by the second, setting $c p_{3}=k_{3}, c p_{0}=k_{1}$ and using the value of $k_{1} / k_{3}$ from (10) gives the result, since

$$
\begin{aligned}
\cot \psi_{3} / 2 & =\left\{\left(1+\cos \psi_{3}\right) /\left(1-\cos \psi_{3}\right)\right\}^{\frac{1}{2}}=\left\{\left(1-\cos ^{2} \psi_{3}\right) /\left(1-\cos \psi_{3}\right)^{2}\right\}^{\frac{1}{2}} \\
& =\sin \psi_{3} /\left(1-\cos \psi_{3}\right)>0 \text { for } 0^{\circ}<\psi_{3}<180^{\circ} .
\end{aligned}
$$

Note 1. Momentum transfer to target. It is sometimes important to know the recoil momentum of the target for a given angle $\psi_{3}$ of projectile scattering in $\Sigma$. From (10) we have

$$
k_{3}=k_{1} /\left\{1+\left(k_{1} / e_{2}\right)(1-a)\right\}, \quad a \equiv a_{3 x}=\cos \psi_{3}
$$

and hence for the k.e. of the target

$$
k_{4}=k_{1}-k_{3}=\left(k_{1} k_{3} / e_{2}\right)(1-a)
$$

Thus $c p_{4}^{2}=\left(E_{4}^{2}-e_{2}^{2}\right)=k_{4}\left(k_{4}+2 e_{2}\right)=k_{4}^{2}+2 e_{2} k_{4}$

$$
\begin{aligned}
& =\left(k_{1}-k_{3}\right)^{2}+2 k_{1} k_{3}(1-a)=k_{1}^{2}+k_{3}^{2}-2 k_{1} k_{3} a \\
& =k_{1}^{2}\left\{1+\left(k_{3} / k_{1}\right)^{2}-2\left(k_{3} / k_{1}\right) a\right\}
\end{aligned}
$$

The desired momentum of the target is therefore 


$$
c p_{4}=k_{1}\left\{1+\left(k_{3} / k_{1}\right)^{2}-2\left(k_{3} / k_{1}\right) \cos \psi_{3}\right\}^{\frac{1}{2}}
$$

where $k_{3} / k_{1}$ is given by (13).

Note 2. Wave length change of the projectile. Setting $k_{1}=h \nu_{1}=h c / \lambda_{1}$, $\mathrm{k}_{3}=h \nu_{3}=h c / \lambda_{3}$ in $(8)$, we obtain

$$
\Delta \lambda=\lambda_{3}-\lambda_{1}=2 \lambda_{2 C} \sin ^{2}\left(\psi_{3} / 2\right)
$$

where $\lambda_{2 \mathrm{C}}=\mathrm{hc} / \mathrm{e}_{2}$ is the Compton wave length of the target. For a scatter of the projectile at right angles with its line of flight

$$
\Delta \lambda=\lambda_{2 C^{*}}
$$

Note 3. In the Compton scattering of a photon on a free electron at rest in $\Sigma$, it is customary to express all energies in units of the electron rest energy $(.511 \mathrm{MeV})$, in which case all formulas of $\$ 31$ are read with $e_{2}=1$. Moreover, the Klein-Nishina differential cross section, governing the energy distribution of the scattered photon, is given in the rest frame $\Sigma$ of the electron, so that the Lorentz transformation is bypassed. The following problem illustrates the method.

A photon of energy $k_{1}=4($ i.e., $2.044 \mathrm{MeV})$ and direction $\Psi_{1}=(2 / 3,2 / 3$, ${ }^{1 / 3)_{A}}$ on given $\Sigma$ axes $A$, collides elastically with a free motionless electron. The energy $k_{z}=4 / 5$ is chosen on the range $4 \geq k_{3} \geq 4 / 9$ by sampling the $K-N$ energy distribution for $k_{1}=4$.

By (8), the corresponding deflection angle is $\psi_{3}=90^{\circ}$. The direction $\Psi_{1}$ being basic for scattering of the photon, $\Psi_{3}$ is itself the latitude angle 
for location of $\Psi_{3}$ about $\Psi_{1}$. If the longitude $\phi$, here assumed uniformly distributed, is chosen as $300^{\circ}$, then $\Omega=(0,1 / 2,-\sqrt{3} / 2)_{A}$ is the auxiliary direction about $X$, and $\left(\Psi_{3}\right)_{A}=D(\Omega)_{A}$ locates $\Psi_{3}$ about $\Psi_{1}$, where $D$ is the matrix of Example (30.1). The final photon momentum is therefore

$$
\mathrm{cP}_{3}=(4 / 5)\left(\Psi_{3}\right)_{A}
$$

on the $\Sigma$ axes $A$. If desired, one may a1so obtain

$$
k_{4}=k_{1}-k_{3}=16 / 5, \quad E_{4}=1+k_{4}=21 / 5, \quad c p_{4}=\sqrt{E_{4}^{2}}-1=4 \sqrt{26 / 5},
$$

and

$$
\mathrm{cP}_{4}=\left(\mathrm{cP}_{1}\right)_{A}-\left(\mathrm{cP}_{3}\right)_{A^{\prime}} \text {, where }\left(\mathrm{cP}_{1}\right)_{A}=4\left(\Psi_{1}\right)_{A} \text {, All energies are con- }
$$

verted to $\mathrm{MeV}$ on multiplication by .511 .

Note 4. The $\mathrm{K}-\mathrm{N}$ differential cross section governing the angular distribution of a photon scattered by a free electron at rest is

$$
s\left(k_{1}, a\right) d a=\pi r^{2}\left(k_{3} / k_{1}\right)^{2}\left\{\left(k_{1} / k_{3}\right)+\left(k_{3} / k_{1}\right)-\left(1-a^{2}\right)\right\} d a c m^{2}
$$

where $k_{1}, k_{3}$ are in units of $.511 \mathrm{MeV}$, and $a=a_{3 x}=\cos \psi_{3}, \psi_{3}$ the photon scattering angle, and $k_{3}=k_{1} /\left\{1+k_{1}(1-a)\right\}$ by (10). Introducing the variable $x=k_{3} / k_{1}$, it can be shown that this implies for $x$ a probability distribution

$$
P(x)=F(x) / F(\xi) \text { on } \xi \equiv 1 /\left(1+2 k_{1}\right) \leq x \leq 1
$$

where

$$
F(x)=f_{x}^{1}\left(x+x^{-1}+a^{2}-1\right) d x, \quad a=1+k_{1}^{-1}-\left(k_{1} x\right)^{-1} . \quad \text { For } 2 \leq k_{1} \leq 202
$$

this can be sampled for $x$ by setting a random number 


$$
\mathbf{r}=\mathrm{F}(\mathrm{x}) / \mathrm{F}(\xi)
$$

and obtaining $x=F^{-1}(r F(\xi))$, which can now be done using an approximation for the inverse function $F^{-1}$. If $k_{1}>202$ a much simpler method exists (LA-4663)

Although we make no use of it, we include the energy

cross section, defined by

$$
s\left(k_{1}, k\right) d k \equiv s\left(k_{1}, a\right) d a
$$

where

$$
\begin{gathered}
a=1+1 / k_{1}-1 / k, \quad d a / d k=1 / k^{2}\left(k_{j} \equiv k_{3}\right), \text { namely } \\
s\left(k_{1}, k\right)=\left(\pi r_{\varepsilon}^{2} / k_{1}^{2}\right)\left\{k^{-2}-\left(2 k_{1}^{-1}+2-k_{1}\right) k^{-1}+\left(k_{1}^{-2}+2 k_{1}^{-1}\right)+k_{1}^{-1} k\right\}
\end{gathered}
$$

Integration on the interval $k_{1} /\left(1+2 k_{1}\right) \leq k \leq k_{1}$ yields the total cross section

$$
s\left(k_{1}\right)=2 \pi r_{\varepsilon}^{2}\left\{2 k_{1}^{-2}+\left(1+k_{1}\right)\left(1+2 k_{1}\right)^{-2}-\left(k_{1}^{-3}+k_{1}^{-2}-2^{-1} k_{1}^{-1}\right) \ln \left(1+2 k_{1}\right)\right\}
$$


32. Elastic collision (TAR, I=2, $e_{1}=0$, polarized case). (A) A plane polarized photon may be characterized in a frame $\Sigma$ by its energy $k_{1}=$ $h \nu_{1} / m(\varepsilon) c^{2}$, direction $\Psi_{1}$, and an electric unit vector $\Delta_{1}$ in the plane $\pi_{1}$ orthogonal to $\Psi_{1}$. Its Klein-Nishina differential cross section for elastic scattering, on a free unpolarized electron at rest in $\Sigma$, into a direction within $\mathrm{d}_{2}$ of $\Psi_{2}$, with an electric vector $\Delta_{2}$, in the plane $\pi_{2}$ orthogonal to $\Psi_{2}$, at angle $\theta$ with $\Delta_{1}$, i.e., with

$$
\Delta_{2} \cdot \Delta_{1}=\cos \theta
$$

is given by

$$
s\left(\Psi_{2}, \Delta_{2}\right) d \Psi_{2}=\left(r^{2} / 2\right)\left(k_{2} / k_{1}\right)^{2}\left\{\frac{1}{2} k_{2}-1+2 \cos ^{2} \Theta\right\} d \Psi_{2} \mathrm{~cm}^{2}
$$

where $k_{2} \equiv k_{2} / k_{1}+k_{1} / k_{2}, \quad k_{2}=k_{1} /\left\{1+k_{1}\left(1-\cos \psi_{2}\right)\right\}$, and $\cos \psi_{2}=\Psi_{2} \cdot \Psi_{1}$.

For a given scatter direction $\Psi_{2}$, one defines two basic directions in the plane $\pi_{2}$ (Fig. 1),

$$
\Delta_{1}^{\perp}=\Psi_{2} \times \Delta_{1} /\left|\Psi_{2} \times \Delta_{1}\right| \quad \Delta_{1}^{\|}=\Delta_{1}^{\perp} \times \Psi_{2}
$$

Note that $\left[\Psi_{2}, \Delta_{1}^{\|}, \Delta_{1}^{\perp}\right]$ form a right handed set of axes, with $\Delta_{1}^{\perp}$ orthogonal to $\Psi_{2}, \Delta_{1}$, and $\Delta_{1}^{\|}$, which are therefore coplanar.

Hence, for an arbitrary $\Delta_{2}$ in $\pi_{2}$, we may write $\Delta_{2}=A+B$, where $A=\left(\Delta_{2} \cdot \Delta_{1}^{\|}\right) \Delta_{1}^{\|}$and $B=\left(\Delta_{2} \cdot \Delta_{1}^{\perp}\right) \Delta_{1}^{\perp}$ are its components on $\Delta_{1}^{\|}, \Delta_{1}^{\perp}$. For this $\Delta_{2}$ we next compute the corresponding 


$$
\begin{aligned}
\cos ^{2} \theta & =\left(\Delta_{1} \cdot \Delta_{2}\right)^{2}=\left(\Delta_{1} \cdot(A+B)\right)^{2}=\left(\Delta_{1} \cdot A\right)^{2} \\
& =\left(\Delta_{2} \cdot \Delta_{1}^{\|}\right)^{2}\left(\Delta_{1} \cdot \Delta_{1}^{\|}\right)^{2}=\left(\Delta_{2} \cdot \Delta_{1}^{\|}\right)^{2}\left\{1-\left(\Psi_{2} \cdot \Delta_{1}\right)^{2}\right\}
\end{aligned}
$$

The final equality results formally from the vector relations

$$
\Delta_{1} \cdot \Delta_{1}^{\|}=\Delta_{1} \cdot\left(\Delta_{1}^{\perp} \times \Psi_{2}\right)=\Delta_{1}^{\perp} \cdot\left(\Psi_{2} x \Delta_{1}\right)=\Delta_{1}^{\perp} \cdot\left(R_{2}^{\frac{1}{2}} \cdot \Delta_{1}^{\perp}\right)=R_{2}^{\frac{1}{2}}
$$

where $R_{2} \equiv\left|\Psi_{2} \times \Delta_{1}\right|^{2}=1-\left(\Psi_{2} \cdot \Delta_{1}\right)^{2}$

In these terms, (1) becomes

$$
s\left(\Psi_{2}, \Delta_{2}\right) \mathrm{d} \Psi_{2}=\left(\mathrm{r}^{2} / 2\right)\left(\mathrm{k}_{2} / \mathrm{k}_{1}\right)^{2}\left\{\frac{1}{2} \mathrm{~K}_{2}-1+2\left(\Delta_{2} \cdot \Delta_{1}^{\|}\right)^{2}\left[1-\left(\Psi_{2} \cdot \Delta_{1}\right)^{2}\right]\right\} \mathrm{d} \Psi_{2}
$$

We assume that, for a stipulated $\Psi_{2}, \Delta_{2}$ must be exactly one of the two basic vectors $\Delta_{1}^{\|}, \Delta_{1}^{\perp}$. From (3), the corresponding cross sections are

$$
\begin{aligned}
s\left(\Psi_{2}, \Delta_{1}\right) d \Psi_{2} & =\left(r^{2} / 2\right)\left(k_{2} / k_{1}\right)^{2}\left\{\frac{1}{2} K_{2}+1-2\left(\Psi_{2} \cdot \Delta_{1}\right)^{2}\right\} d \Psi_{2} \\
& \geq s\left(\Psi_{2}, \Delta_{1}^{\perp}\right) d \Psi_{2}=\left(r^{2} / 2\right)\left(k_{2} / k_{1}\right)^{2}\left\{\frac{1}{2} K_{2}-1\right\} d \Psi_{2}
\end{aligned}
$$

The sum of the two is the cross section for $\Psi_{2}$ scattering:

$$
s\left(\Psi_{2}\right) d \Psi_{2}=\left(r^{2} / 2\right)\left(k_{2} / k_{1}\right)^{2}\left\{k_{2}-2\left(\Psi_{2} \cdot \Delta_{1}\right)^{2}\right\} d \Psi_{2}
$$

Introducing spherical coordinates $\psi_{2}, \phi_{2}$ relative to axes $\bar{A}$ with $\bar{X}=\Psi_{1}, \bar{Y}=\Delta_{1}$ (Fig. 1), we write 


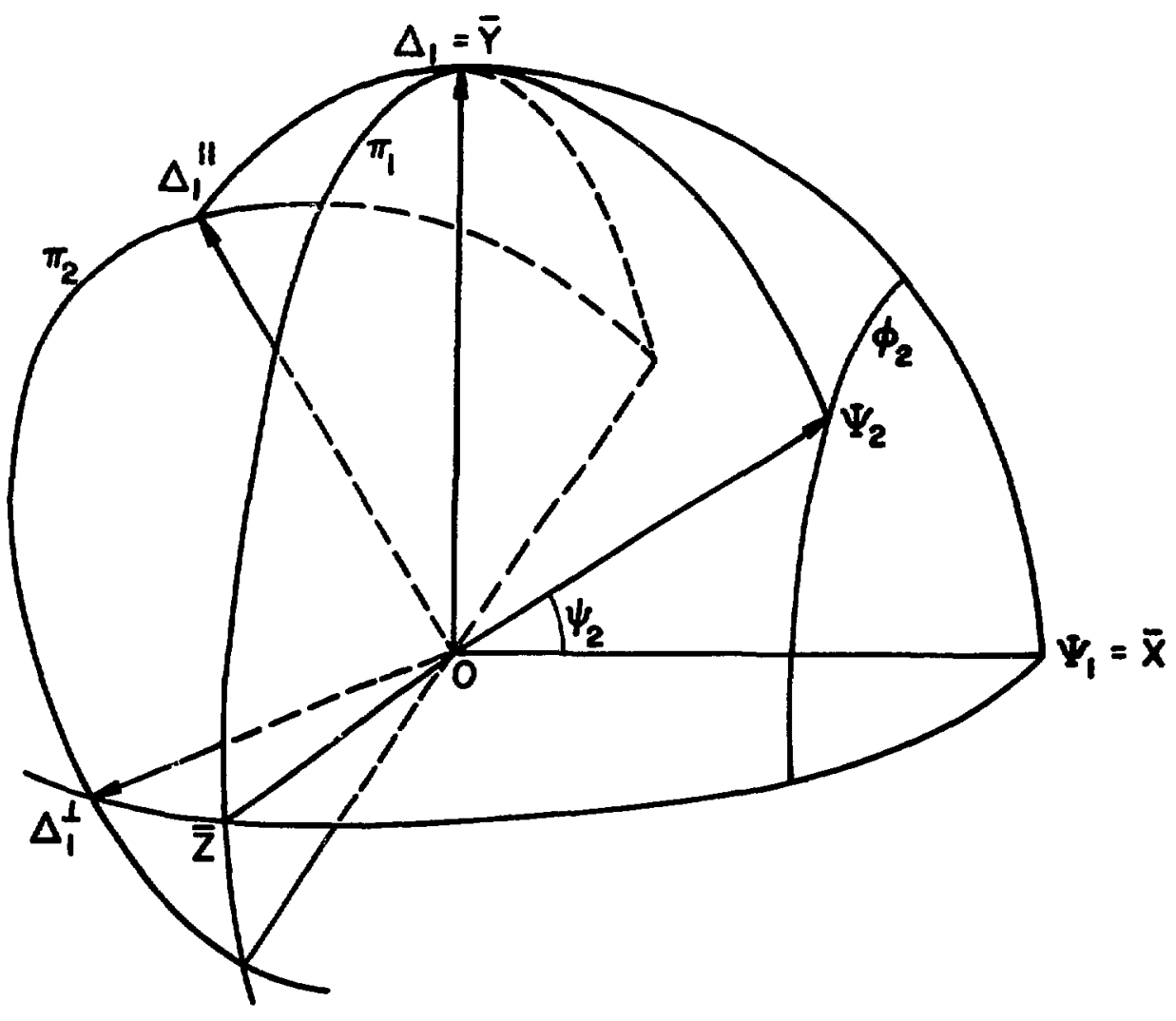

Fig. 1

Polarized Scattering 


$$
\Psi_{2}=\left(\cos \psi_{2}, \sin \psi_{2} \cos \phi_{2}, \sin \psi_{2} \sin \phi_{2}\right)_{\bar{A}}, \Delta_{1}=(0,1,0)_{\bar{A}}
$$

For these coordinates (5) reads

$$
\begin{aligned}
s\left(\Psi_{2}\right) d \Psi_{2} & \equiv s\left(\psi_{2}, \phi_{2}\right) \sin \psi_{2} d \psi_{2} d \phi_{2} \\
& =\left(r^{2} / 2\right)\left(k_{2} / k_{1}\right)^{2}\left\{k_{2}-2 \sin ^{2} \psi_{2} \cos ^{2} \phi_{2}\right\} \sin \psi_{2} d \psi_{2} d \phi_{2}
\end{aligned}
$$

where we note the non-uniformity on the $\Psi_{2}$-cone.

Integration of (7) on $0 \leq \phi_{2} \leq 2 \pi$ then gives

$$
s\left(\psi_{2}\right) \sin \psi_{2} d \psi_{2}=\left(\pi r^{2}\right)\left(k_{2} / k_{1}\right)^{2}\left\{k_{2}-\sin ^{2} \psi_{2}\right\} \sin \psi_{2} d \psi_{2}
$$

as the cross section for scattering within $\mathrm{d} \Psi_{2}$ of the $\Psi_{2}$-cone about $\Psi_{1}$. This is identical with the $\mathrm{K}-\mathrm{N}$ cross section of Note 31.4 , and the energy $\mathrm{k}_{2}$ and deflection $\psi_{2}$ may be sampled just as indicated there. (The index 3 has been replaced by 2 in the present section.)

B. Simple Monte Carlo. Granting the "either $\Delta_{1}^{\|}$or $\Delta_{1}^{1}$ " assumption, one might follow a single $\left(k_{1}, \Psi_{1}, \Delta_{1}\right)$-photon through a collision thus:

a. From $k_{1}$, obtain $k_{2}$ and $\cos \psi_{2}$ for the scattered photon as in Note 31.4 .

b. $\cos \Phi_{2}$ is then obtained from (7) by rejection technique applied to the rectangle enclosing the curve $f\left(\phi_{2}\right)=k_{2}-2 \sin ^{2} \psi_{2} \cos ^{2} \phi_{2}$, $0 \leq \phi_{2} \leq 2 \pi \quad$ (See Notes 1-4).

c. $\left(\Psi_{2}\right)_{A}$ is next computed from (6).

d. By (4), the relative probability of $\Delta_{2}=\Delta_{1}^{1}$ is $\mathrm{p}^{1}=\left(\frac{1}{2} \mathrm{~K}_{1}-1\right) /\left\{\mathrm{K}_{2}-2 \sin ^{2} \psi_{2} \cos ^{2} \phi_{2}\right\}$ from which the alternatives $\Delta_{2}=\Delta_{1}^{\perp}$ and $\Delta_{2}=\Delta_{1}^{\|}$may be sampled. 
e. The $\bar{A}$ coordinates of the selected $\Delta_{2}$ are found from (6), (2).

f. If $\left(\Psi_{1}\right)_{A},\left(\Delta_{1}\right)_{A}$ are the incident vectors on given $\Sigma$ axes $A$, then the rotation $\delta_{2}$ of App. I. Cor. 3, with matrix

$$
D_{2}=\left[\left(\Psi_{1}\right)_{A},\left(\Delta_{1}\right)_{A},\left(\Psi_{1}\right)_{A} x\left(\Delta_{1}\right)_{A}\right]
$$

takes axes $A$ into axes $\bar{A}$, on which $\Psi_{2}$ and $\Delta_{2}$ are known from (c) and (e). Hence $\left(\Psi_{2}\right)_{A}=D_{2}\left(\Psi_{2}\right)_{\bar{A}},\left(\Delta_{2}\right)_{A}=D_{2}\left(\Delta_{2}\right)_{A}$ give the direction and electric vector of the scatter photon on the original $\sum$ axes A. (App. I, Theorem $1(b)$ ).

C. Stokes Method. If one is concerned only with the changes of direction $\Psi$ of a mono-energetic photon beam, upon which its energy depends, and not with its $\Delta$-vector composition, the method of Stokes parameters is indicated. Such a beam is characterized by the parameters $k_{1}, \Psi_{1}, \Delta_{1}^{0}, Q_{1}, U_{1}$, where $k_{1}$ and $\Psi_{1}$ are its energy and direction, $\Delta_{1}^{0}$ is the "Stokes vector," and $Q_{1}, U_{1}$ the "Stokes parameters." The latter are two numbers, (reflecting the $\Delta$-composition of the beam) which suffice to determine its probability $P_{1}\left(\Psi_{2}\right) d \Psi_{2}$ of scattering direction, and also serve to determine, for that part of the bean which scatters at $\Psi_{2}$, the accompanying Stokes parameters $\mathrm{Q}_{2}, \mathrm{U}_{2}$ required for its next collision.

A version of the method due to G. I. Bell is given in part (D). The present part is an attempt to make the method plausible heuristically. Consider then a composite beam of $\left(k_{1}, \Psi_{1}\right)$-photons, $s_{1}^{i}$ denoting the fraction $\left(\Sigma S_{1}^{i}=1\right)$ having their $\Delta_{1}$ vector $\Delta_{1}^{i}(i=1, \ldots, N)$ at angle $\eta_{1}^{i}$ from a specified direction $\Delta_{1}^{0}$ (Stokes vector) in the plane $\pi_{1}$ orthogonal to $\Psi_{1}$ (Fig. 2) . 


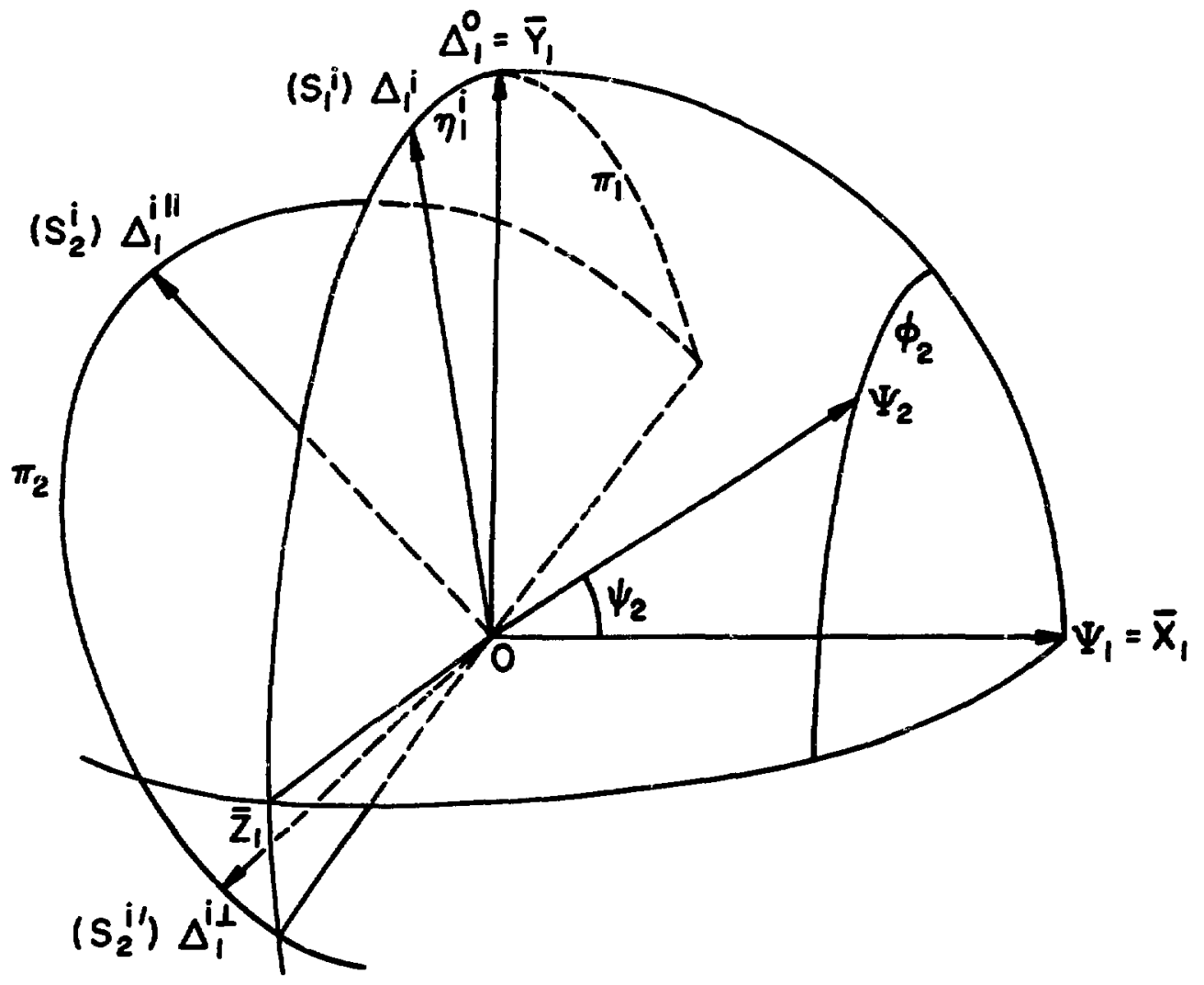

Fig. 2 .

Stokes Vector $\Delta_{1}^{0}$ 
We now fix upon a particular direction $\Psi_{2}$ of scatter with coordinates

$$
\Psi_{2}=\left(\cos \psi_{2}, \sin \psi_{2} \cos \phi_{2}, \sin \psi_{2} \sin \phi_{2}\right)_{A_{1}}
$$

on the axes $\bar{A}_{1}$, with $\bar{X}_{1}=\psi_{1}$, and $\bar{Y}_{1}=\Delta_{1}^{0}$ the given Stokes vector. On these axes we have also

$$
\Delta_{1}^{i}=\left(0, \cos n_{1}^{i}, \sin n_{1}^{i}\right)_{1}
$$

wherice

$$
\Psi_{2} \cdot \Delta_{1}^{i}=\sin \psi_{2} \cos \left(\phi_{2}-n_{1}^{i}\right)
$$

For the fixed $\Psi_{2}$, and each $\Delta_{1}^{i}$, there are then two possible resulting $\Delta$-vectors,

$$
\Delta_{1}^{i \perp}=\Psi_{2} \times \Delta_{1}^{i} /\left|\Psi_{2} \times \Delta_{1}^{i}\right| \text { and } \Delta_{1}^{i \|}=\Delta_{1}^{i \perp} \times \Psi_{2}
$$

for the scattered photon. Their associated probability density functions are seen from (4) to be

$$
\begin{aligned}
& \mathrm{p}\left(\Psi_{2}, \Delta_{1}^{i \|}\right) \mathrm{d} \Psi_{2}=\left\{\frac{1}{2} \mathrm{~K}_{2}+1-2\left(\Psi_{2} \cdot \Delta_{1}^{i}\right)^{2}\right\} \mathrm{d} \Psi_{2} / D_{2} \\
& \mathrm{p}\left(\Psi_{2}, \Delta_{1}^{i \perp}\right) \mathrm{d} \Psi_{2}=\left\{\frac{1}{2} \mathrm{~K}_{2}-1\right\} \mathrm{d} \Psi_{2} / D_{2}
\end{aligned}
$$

where $D_{2}=s_{1} /\left(r^{2} / 2\right)\left(k_{2} / k_{1}\right)^{2}$ is a function of $\psi_{2}$, and $s_{1}$ is the total cross section at incident energy $k_{1}$.

Hence the probability of a beam photon having $\Delta_{1}=\Delta_{1}^{i}$, scattering to $\Psi_{2}$, and having $\Delta_{2}=\Delta_{1}^{i \|}$ or $\Delta_{2}=\Delta_{1}^{i \perp}$ is 


$$
\mathrm{s}_{1}^{\mathrm{i}} \mathrm{p}\left(\Psi_{2}, \Delta_{1}^{\mathrm{i} l}\right) \mathrm{d} \Psi_{2} \quad \text { or } \quad \mathrm{s}_{1}^{\mathrm{i}} \mathrm{p}\left(\Psi_{2}, \Delta_{1}^{\mathrm{i} \perp}\right) \mathrm{d} \Psi_{2}
$$

The sum of the two is the probability of a beam photon having $\Delta_{1}=\Delta_{1}^{i}$ and scattering to $\Psi_{2}$ namely (cf. (9)),

$$
\begin{aligned}
& s_{1}^{i}\left\{k_{2}-2\left(\Psi_{2} \cdot \Delta_{1}^{i}\right)\right\} d \Psi_{2} / D_{2} \\
& \quad=s_{1}^{i}\left\{k_{2}-2 \sin ^{2} \Psi_{2} \cos ^{2}\left(\phi_{2}-\eta_{1}^{i}\right)\right\} d \Psi_{2} / D_{2}
\end{aligned}
$$

Suming this on $i$ yields the total probability of a beam photon scattering to $\Psi_{2}$,

$$
\begin{aligned}
& P_{1}\left(\Psi_{2}\right) d \Psi_{2}=\left\{K_{2}-2 \sin ^{2} \Psi_{2} \sum s_{1}^{i} \cos ^{2}\left(\phi_{2}-\eta_{1}^{i}\right)\right\} d \Psi_{2} / D_{2} \\
& \quad=\left\{K_{2}-\sin ^{2} \Psi_{2}-\sin ^{2} \Psi_{2} \sum s_{1}^{i} \cos 2\left(\phi_{2}-\eta_{1}^{i}\right)\right\} d \Psi_{2} j D_{2}
\end{aligned}
$$

Therefore we may write

$$
\begin{aligned}
& P_{1}\left(\Psi_{2}\right) d \Psi_{2}=\rho_{2}\left(\Psi_{2}\right) d \Psi_{2} / D_{2} \\
& \rho_{2}\left(\Psi_{2}\right)=k_{2}-\sin ^{2} \Psi_{2}-\sin ^{2} \Psi_{2} Q_{2}\left(\phi_{2}\right) \\
& Q_{2}\left(\phi_{2}\right)=Q_{1} \cos 2 \phi_{2}+U_{1} \sin 2 \phi_{2} \\
& Q_{1}=\Sigma s_{1}^{i} \cos 2 n_{1}^{i} \quad U_{1}=\Sigma s_{1}^{i} \sin 2 n_{1}^{i}
\end{aligned}
$$

Here, $Q_{1}$ and $U_{1}$ are the Stokes parameters, serving to determine 
$P_{1}\left(\Psi_{2}\right) d \Psi_{2}$ for the composite beam. For later use, we also define here a companion to $Q_{2}\left(\phi_{2}\right)$, namely

$$
U_{2}\left(\phi_{2}\right)=-Q_{1} \sin 2 \phi_{2}+U_{1} \cos 2 \phi_{2}
$$

It remains to compute the Stokes parameters for the $\Psi_{2}$ scattered beam. Its fractional composition or $\Delta_{2}$ vectors is obtained as follows. The probabilities in (12) give the fraction of photons in the $\Psi_{1}$ beam which belonged to its i-th component and scattered to $\Psi_{2}$, and their sum over $i$ is the total fraction scattered to $\Psi_{2}$, namely $P_{1}\left(\Psi_{2}\right) d \Psi_{2}=\rho_{2}\left(\Psi_{2}\right) d \Psi_{2} / D_{2}$, as in (14). Dividing (12) by (14) and using (11) shows the $\Psi_{2}$ scattered beam to have the $\Delta_{2}$ fractional composition

$$
\begin{aligned}
& s_{2}^{i}=\left(s_{1}^{i} / \rho_{2}\left(\Psi_{2}\right)\left\{\frac{1}{2} K_{2}+1-2\left(\Psi_{2} \cdot \Delta_{1}^{i}\right)^{2}\right\}\right. \\
& S_{2}^{i_{1}}=\left(S_{1}^{i} / \rho_{2}\left(\Psi_{2}\right)\right)\left\{\frac{1}{2} K_{2}-1\right\}
\end{aligned}
$$

arising from $\Delta_{1}^{\prime \prime}$ and $\Delta_{1}^{\perp}$ components of the $\Psi_{1}$ beam, where $\Sigma\left(S_{2}^{i}+S_{2}^{i}\right)=1$.

Now the argument establishing (14-17) was quite general, and we may interpret it for the next scattering, once we have referred the $\Delta_{2}$-vectors to a basic Stokes vector $\Delta_{2}^{0}$ in their plane $\pi_{2}$. This we take to be the vector

$$
\begin{aligned}
\Delta_{2}^{\circ} & =\left(\cos \left(\psi_{2}+90^{\circ}\right), \sin \left(\psi_{2}+90^{\circ}\right) \cos \phi_{2}, \sin \left(\psi_{2}+90^{\circ}\right) \sin \phi_{2}\right) \bar{A}_{1} \\
& =\left(-\sin \psi_{2}, \cos \psi_{2} \cos \phi_{2}, \cos \psi_{2} \sin \phi_{2}\right) \bar{A}_{1}
\end{aligned}
$$

referred so the $\Sigma$ axes $A_{1}$. Note that $\Delta_{2}^{0}$ depends on $\Psi_{2}$ alone, its position in the plane $\pi_{2}$ being understood in Fig. 2 . 
Accordingly, we define $\eta_{2}^{i}$ and $r_{1}^{i},=\eta_{2}^{i}+90^{\circ}$ as the angles from $\Delta_{2}^{0}$ to the vectors $e_{1}^{i l l}$ and $e_{1}^{i 1}$, respectively. The new source then appears as in Fig. 3, which is the exact analogue of Fig. 2.

It is clear that we may write at once the probability for $\Psi_{3}$-scatter of the new $\Psi_{2}$ beam in the form of $(14-17)$ :

$$
\begin{aligned}
& \mathrm{F}_{2}\left(\Psi_{3}\right) \mathrm{d} \Psi_{3}=\rho_{3}\left(\Psi_{3}\right) \mathrm{d} \Psi_{3} / D_{3} \\
& \rho_{3}\left(\Psi_{3}\right)=K_{3}-\sin ^{2} \psi_{3}-\sin ^{2} \psi_{3} Q_{3}\left(\phi_{3}\right) \\
& Q_{3}\left(\phi_{3}\right)=Q_{2} \cos 2 \phi_{3}+U_{2} \sin 2 \phi_{3} \\
& Q_{2}=\sum s_{2}^{i} \cos 2 n_{2}^{i}+\sum s_{2}^{i} \cos 2 n_{2}^{i} \\
& =\Sigma\left(s_{2}^{i},-s_{2}^{i}\right) \cos 2 n_{2}^{i} \\
& u_{2}=\Sigma s_{2}^{i} \sin 2 n_{2}^{i}+\sum s_{2}^{i}, \sin 2 n_{2}^{i} \\
& =\Sigma\left(s_{2}^{i},-s_{2}^{i}\right) \sin 2 n_{2}^{i}
\end{aligned}
$$

The $Q_{2}, U_{2}$ are the Stokes parameters for the new $\Psi_{2}$ beam, determining the probability $P_{2}\left(\Psi_{3}\right) d \Psi_{3}$ for the next scattering. From (19), we see that

$$
s_{2}^{i},-s_{2}^{i}=-2 s_{1}^{i} R_{2}^{i} / \rho_{2}\left(\Psi_{2}\right)
$$

where we have defined (cf. (9))

$$
R_{2}^{i}=1-\left(\Psi_{2} \cdot \Delta_{1}^{i}\right)^{2} \equiv\left|\Psi_{2} \times \Delta_{1}^{i}\right|^{2}=1-\sin ^{2} \Psi_{2} \cos ^{2}\left(\phi_{2}-\eta_{1}^{i}\right)
$$




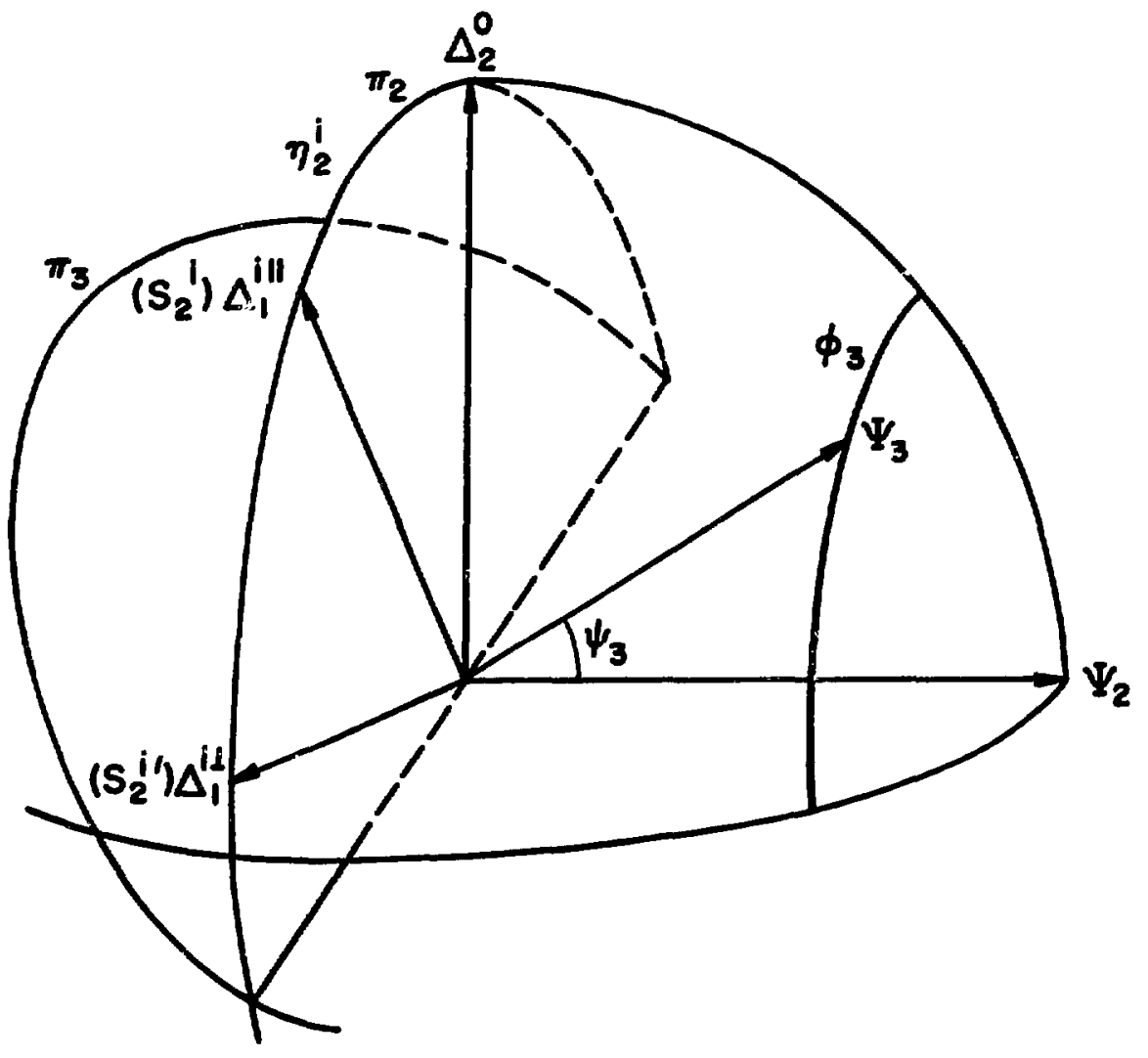

Fig. 3.

The $\Psi_{2}$ beam 
Hence the new Stokes parameters in (24) may be written as

$$
\begin{aligned}
& Q_{2}=\left(-2 / \rho_{2}\left(\Psi_{2}\right)\right) \Sigma S_{1}^{i} R_{2}^{i} \cos 2 n_{2}^{i}, \\
& U_{2}=\left(-2 / \rho_{2}\left(\psi_{2}\right)\right) \Sigma s_{1}^{i} R_{2}^{i} \sin 2 n_{2}^{i},
\end{aligned}
$$

Now it is a remarkable fact that $Q_{2}, U_{2}$ are related to the previous $Q_{1}, U_{1}$ by functions depending only on $\psi_{2}$ and $\phi_{2}$. To show how this comes about, we must first relate the angles $\eta_{2}^{i}$ and $\eta_{1}^{i}$. In fact,

$$
\cos \eta_{2}^{i}, \equiv \Delta_{2}^{0} \cdot \Delta_{1}^{i \perp} \equiv \Delta_{2}^{0} \cdot\left(\Psi_{2} \times \Delta_{1}^{i}\right) /\left(R_{2}^{i}\right)^{\frac{1}{2}} \equiv \Delta_{1}^{i} \cdot\left(\Delta_{2}^{0} \times \Psi_{2}\right) /\left(R_{2}^{i}\right)^{\frac{1}{2}}
$$

where we have used (10) and (25). From the coordinates of $\Psi_{2}, \Delta_{1}^{i}, \Delta_{2}^{0}$ on axes $A_{1}$ in (8), (8a), (20) one computes $\Delta_{2}^{0} \times \Psi_{2}=\left(0, \sin \phi_{2},-\cos \phi_{2}\right) A_{1}$, and hence the relation

$$
\cos \eta_{2}^{i_{1}}=\sin \left(\phi_{2}-\eta_{1}^{i}\right) /\left(R_{2}^{i}\right)^{\frac{1}{2}}
$$

From this and (25) we see that

$$
\begin{aligned}
R_{2}^{i} \cos 2 n_{2}^{i}, & \equiv R_{2}^{i}\left(2 \cos ^{2} \eta_{2}^{i}-1\right)=2 \sin ^{2}\left(\phi_{2}-\eta_{1}^{i}\right)-1+\sin ^{2} \psi_{2} \cos ^{2}\left(\phi_{2}-\eta_{1}^{i}\right) \\
& \equiv 1-\left(1+\cos ^{2} \psi_{2}\right) \cos ^{2}\left(\phi_{2}-\eta_{1}^{i}\right) \\
& \equiv 1-\frac{1}{2}\left(1+\cos ^{2} \psi_{2}\right)\left(1+\cos 2\left(\phi_{2}-\eta_{1}^{i}\right)\right) \\
& \equiv \frac{1}{2}\left\{\sin ^{2} \psi_{2}-\left(1+\cos ^{2} \psi_{2}\right)\left(\cos 2 \phi_{2} \cos 2 \eta_{1}^{i}+\sin 2 \phi_{2} \sin 2 \eta_{1}^{i}\right)\right.
\end{aligned}
$$

Substitution in (26) then gives $Q_{2}=$ 
$-206-$

$$
\begin{aligned}
& \left(-1 / \rho_{2}\left(\Psi_{2}\right)\right)\left\{\sin ^{2} \psi_{2}-\left(1+\cos ^{2} \psi_{2}\right)\left(\cos 2 \phi_{2} \sum s_{1}^{i} \cos 2 \eta_{1}^{i}+\sin 2 \phi_{2} \sum s_{1}^{i} \sin \eta_{1}^{i}\right)\right\} \\
& =\left\{-\sin ^{2} \psi_{2}+\left(1+\cos ^{2} \psi_{2}\right)\left(Q_{1} \cos 2 \phi_{2}+U_{1} \sin 2 \phi_{2}\right)\right\} / \rho_{2}\left(\Psi_{2}\right) \\
& =\left\{-\sin ^{2} \psi_{2}+\left(1+\cos ^{2} \psi_{2}\right) Q_{2}\left(\phi_{2}\right)\right\} / \rho_{2}\left(\Psi_{2}\right)
\end{aligned}
$$

which expresses $Q_{2}$ in terms of $Q_{1}, U_{1}$ and $\psi_{2}, \phi_{2}$.

For $U_{2}$ we require $\sin n_{2}^{i_{1}}=\sin \left(n_{2}^{i}+90^{\circ}\right)=\cos n_{2}^{i}=$

$$
\begin{aligned}
& \Delta_{2}^{0} \cdot \Delta_{1}^{i \|}=\Delta_{2}^{0} \cdot\left(\Delta_{1}^{i \perp} \times \Psi_{2}\right)=\Delta_{1}^{i \perp} \cdot\left(\Psi_{2} x \Delta_{2}^{0}\right)=\left(\Psi_{2} x \Delta_{1}^{i}\right) \cdot\left(\Psi_{2} x \Delta_{2}^{0}\right) /\left(R_{2}^{i}\right)^{\frac{1}{2}} \\
& =\left(\Delta_{1}^{i} x \Psi_{2}\right) \cdot\left(\Delta_{2}^{0} \times \Psi_{2}\right) /\left(R_{2}^{i}\right)^{\frac{1}{2}} \text {. We have found }\left(\Delta_{2}^{0} x \Psi_{2}\right)=\left(0, \sin \phi_{2},-\cos \phi_{2}\right) \bar{A}_{1}
\end{aligned}
$$

above, and (8), (Ba) show that

$$
\begin{aligned}
& \left(\Delta_{1}^{i} x \Psi_{2}\right)=\left({ }^{*}, \cos \psi_{2} \sin \eta_{1}^{i},-\cos \psi_{2} \cos \eta_{1}^{i}\right)_{A_{1}} \text {, so that } \\
& \quad \sin \eta_{2}^{i},=\cos \psi_{2} \cos \left(\phi_{2}-\eta_{1}^{i}\right) /\left(R_{2}^{i}\right)^{\frac{1}{2}} .
\end{aligned}
$$

This and (27) imply that

$$
\begin{aligned}
\mathrm{R}_{2}^{\mathrm{i}} \sin 2 n_{2}^{i}, & \equiv 2 \cos \psi_{2} \cos \left(\phi_{2}-n_{1}^{i}\right) \sin \left(\phi_{2}-n_{1}^{i}\right) \\
& \equiv \cos \psi_{2} \sin 2\left(\phi_{2}-\eta_{1}^{i}\right) \\
& =\cos \Psi_{2}\left(\sin 2 \phi_{2} \cos 2 n_{1}^{i}-\cos 2 \phi_{2} \sin 2 \pi_{1}^{i}\right)
\end{aligned}
$$

Hence from (26) we obtain

$$
\begin{aligned}
\mathrm{U}_{2} & =\left(-2 / \rho_{2}\left(\Psi_{2}\right)\right) \cos \Psi_{2}\left(\mathrm{Q}_{1} \sin 2 \phi_{2}-\mathrm{U}_{1} \cos 2 \phi_{2}\right) \\
& =2 \cos \Psi_{2} \mathrm{U}\left(\Phi_{2}\right) / \rho_{2}\left(\Psi_{2}\right)
\end{aligned}
$$


Thus (28) and (30) express the Stokes parameters $Q_{2}, U_{2}$ of the $\Psi_{2}$ scattered bean in terms of the parameters $Q_{1}, U_{1}$ of the parent $\Psi_{1}$ bean, and the spherical coordinates oi $\Psi_{2}$ relative to the axes $A_{1}$, with $\bar{X}_{1}=\Psi_{1}$, and $\bar{y}_{1}=\Delta_{1}^{0}$ the Stokes vector of $\Psi_{1}$.

D. Stokes method. Beam parameters $k, \Psi, \Delta^{0}, Q, U$. Initial values must be assigned at the source. A uniformly polarized beam has $Q=0$, $\mathrm{U}=0, \Delta^{0}$ arbitrary, while a "pure" beam has $Q=1, U=0$ for a suitable $\Delta$

One follows a $k_{1}, \Psi_{1}, \Delta_{1}^{0}, Q_{1}, U_{1}$ collision thus:

a. One obtains $k_{2}, \cos \psi_{2}$ as in Note 31.4 .

b. $\phi_{2}$ is found from (15) by rejection technique applied to the rectangle enclosing the curve $f\left(\phi_{2}\right)=k_{2}-\sin ^{2} \psi_{2}-$ $\sin ^{2} \psi_{2} Q_{2}\left(\phi_{2}\right)$. (See Notes 1-4.)

c. $\left(\Psi_{2}\right)_{A_{1}}$ and $\left(\Delta_{2}^{0}\right) \bar{A}_{1}$ are obtained from (8) and $(20)$, on the axes $\bar{A}_{1}$ d. The new direction $\Psi_{2}$ and Stokes vector $\Delta_{2}^{0}$ on the original axes $A$ are

$$
\left(\Psi_{2}\right)_{A}=D_{2}\left(\Psi_{2}\right)_{A_{1}}, \quad\left(\Delta_{2}^{0}\right)_{A}=D_{2}\left(\Delta_{2}^{0}\right)_{A_{1}}
$$

where

$$
D_{2}=\left[\begin{array}{lll}
\left(\Psi_{1}\right)_{A}, & \left.\left(\Delta_{1}^{0}\right)_{A}, \quad\left(\Psi_{1}\right)_{A} \times\left(\Delta_{1}^{0}\right)_{A}\right]
\end{array}\right.
$$

(See App. I, Theorem 1(b) and Corollary 3.)

e. New Stokes parameters $Q_{2}, U_{2}$ for the $\Psi_{2}$ scattered beam are computed from (28), (30).

These steps are seen to produce the new parameters $k_{2}, \Psi_{2}, \Delta_{2}^{0}, Q_{2}, U_{2}$ 
Note 1. If a probability density function $p(x) d x$ on $[a, b]$ is of form $p(x) d x=K f(x) d x$ where $K>0$ is a constant, and $m \leq f(x) \leq M$, then a correctly distributed $x$ is obtained by successively "throwing" pairs of random numbers $\left(r, r^{\prime}\right)$ on $(0,1]$ and accepting the first $x \equiv a+(b-a) r$ for which $r^{\prime} \leq \frac{f(x)}{M}$, the "efficiency" of the method being

$$
E=\int_{a}^{b} f(x) d x / M(b-a) \geq \int_{a}^{b}(f(x)-m) d x /(M-m)(b-a)
$$

Note 2. For the function $f\left(\phi_{2}\right)=k_{2}-\angle \sin ^{2} \psi_{2} \cos ^{2} \phi_{2}$ on $[0,2 \pi]$, we have $M=K_{2}, m=K_{2}-2 \sin ^{2} \psi_{2}, b-a=2 \pi$, and an efficiency

$$
E \geq \int_{0}^{2 \pi}\left(1-\cos ^{2} \phi_{2}\right) d \phi_{2} / 2 \pi=\int_{0}^{2 \pi} \sin ^{2} \phi_{2} d \phi_{2} / 2 \pi=\frac{1}{2} \text {. }
$$

Note 3. For the function

$$
f\left(\phi_{2}\right)=K_{2}-\sin ^{2} \psi_{2}-\sin ^{2} \psi_{2}\left\{Q_{1} \cos 2 \phi_{2}+U_{1} \sin 2 \phi_{2}\right\} \text { on }[0,2 \pi]
$$

we may write $f\left(\phi_{2}\right)=A-B \cos 2\left(\phi_{2}-\phi_{0}\right)$, where $A=k_{2}-\sin ^{2} \psi_{2}$ and $B=\sin ^{2} \psi_{2}\left(Q_{1}^{2}+U_{1}^{2}\right)^{\frac{1}{2}}$, with $\phi_{0}$ defined by $\cos 2 \phi_{0}=Q_{1} /\left(Q_{1}^{2}+U_{1}^{2}\right)^{\frac{1}{2}}$, $\sin 2 \phi_{0}=U_{1} /\left(Q_{1}^{2}+U_{1}^{2}\right)^{\frac{1}{2}}, 0 \leq \phi_{0} \leq 180^{\circ}$.

From this form, we see that $M=A+B, m=A-B$, and again $E \geq \frac{1}{2}$.

Note 4. In applying the method of Note 1 to the functions of Notes 2, 3, it should be observed that, instead of "throwing" $\phi_{2}=2 \pi r$ and then computing $\cos ^{2} \phi_{2}$ or $\cos 2 \phi_{2}$, sin $2 \phi_{2}$, one may use von Neumann's device of "throwing" directly for the $\cos \phi_{2}$, sin $\phi_{2}$ of a uniformly distributed angle $\phi_{2}$ on $(0,2 \pi)$, and then computing $\cos ^{2} \phi_{2}$, or $\cos 2 \phi_{2}\left(=\cos ^{2} \phi_{2}-\sin ^{2} \phi_{2}\right), \sin 2 \phi_{2}\left(=2 \sin \phi_{2}\right.$ $\cos \phi_{2}$ ). The device referred to (itself a rejection technique) goes as follows: Of a sequence of random pairs $\left(r_{1}, r_{2}\right)$ one accepts the first for which $S=x^{2}+y^{2} \leq 1$, where $x=2 r_{1}-1, y=r_{2}$, and sets $\cos \phi_{2}=\left(x^{2}-y^{2}\right) / S$, 
$\sin \phi_{2}=2 x y / S$. The efficiency here is $\pi / 4$. Note that $\cos \phi_{2}, \sin \phi_{2}$ are themselves required in subsequent computation.

Note 5. The argument in part (C) does not require that the vectors $\Delta_{1}^{i}$ be distinct, e.g., in one $90^{\circ}$ scattering all $\Delta$-vectors present in a beam collapse to four, at most.

Note 6. The "straightforward" method of part (B) based on the "either $\Delta_{1}^{\| \prime}$ or $\Delta_{1}^{1}$ " assumption has not been tried and may not work. Neve:-theless, as shown in $(C)$, the assumption seems to lead to the correct $Q, U$ transformation.

Note 7. It is easy to verify that the $\psi_{2}$-marginal density of $P_{1}\left(\dddot{Y}_{2}\right) d \Psi_{2}$ in (13) is the density defined by the $\mathrm{K}-\mathrm{N}$ cross section of Note 31.4. This justifies step (a) of part (D).

Note 8. $90^{\circ}-90^{\circ}$ scattering. As an exercise in the use of Stokes parameters, we consider an initial uniformly polarized beam with parameters

$$
\mathrm{k}_{1}=\mathrm{k}_{0}, \quad \Psi_{1}=(1,0,0)_{A} \quad \Delta_{1}^{0}=(0,1,0)_{A} \quad Q_{1}=0 \quad \mathrm{U}_{1}=0
$$

which on first collision scatters into the direction $\Psi_{2}=(0,0,1)_{A}$ and then on second collision scatters into the XY-plane. Following the method of part (D), we obtain

$$
\begin{aligned}
& \text { a. } \psi_{2}=90^{\circ} \text { by assumption, } \cos \psi_{2}=0, k_{2}=k_{0} /\left(1+k_{0}\right), \\
& K_{2}=k_{2} / k_{1}+k_{1} / k_{2}=\left(2+2 k_{0}+k_{0}^{2}\right) /\left(1+k_{0}\right) \\
& \text { b. } \phi_{2}=90^{\circ} \text { by assumption. } \\
& \text { c. } \Psi_{2}=(0,0,1)_{A_{1}}, \Delta_{2}^{o}=(-1,0,0)_{A} A_{1} \\
& \text { d. } D_{2}=I, \quad \Psi_{2}=(0,0,1)_{A}, \quad \Delta_{2}^{0}=(-1,0,0)_{A}
\end{aligned}
$$


e. From (15-17), $Q_{1}=0, \mathrm{U}_{1}=0, \mathrm{Q}_{2}\left(\phi_{2}\right)=0, \rho_{2}\left(\Psi_{2}\right)=$

$$
\begin{aligned}
& \mathrm{K}_{2}-1=\left(1+\mathrm{k}_{0}+\mathrm{k}_{0}^{2}\right) /\left(1+\mathrm{k}_{0}\right), \text { and from }(28),(30), \\
& Q_{2}=-1 / \mathrm{\rho}_{2}\left(\Psi_{2}\right)=-\left(1+\mathrm{k}_{0}\right) /\left(1+\mathrm{k}_{0}+\mathrm{k}_{0}^{2}\right), \quad U_{2}=0
\end{aligned}
$$

Advancing subscripts we assign to the once scattered beam the new parameters

$$
k_{1}=k_{0} /\left(1+k_{0}\right), \Psi_{1}=(0,0,1)_{A}, \Delta_{1}^{0}=(-1,0,0)_{A}, \quad Q_{1}=-\left(1+k_{0}\right) /\left(1+k_{0}+k_{0}^{2}\right), U_{1}=0
$$

and repeat the procedure:

$$
\begin{aligned}
& a^{\prime} \cdot \psi_{2}=90^{\circ} \text { by assumption, } \cos \psi_{2}=0, k_{2}=k_{0} /\left(1+2 k_{0}\right) \\
& \mathrm{K}_{2}=\left(2+6 \mathrm{k}_{\mathrm{o}}+5 \mathrm{k}_{\mathrm{o}}^{2}\right) /\left(1+\mathrm{k}_{0}\right)\left(1+2 \mathrm{k}_{\mathrm{o}}\right) \\
& b^{\prime} \text {. From (15-17), we have } Q_{2}\left(\phi_{2}\right)=Q_{1} \cos 2 \phi_{2} \text {, so } \\
& \rho_{2}\left(\Psi_{2}\right)=K_{2}-1-Q_{1} \cos 2 \phi_{2}=K_{2}-1+Q_{1}\left(1-2 \cos ^{2} \phi_{2}\right) \\
& =K_{2}-1+Q_{1}-2 Q_{1} \cos ^{2} \phi_{2}
\end{aligned}
$$

Substitution of $K_{2}$ and $Q_{1}$ shows that

$$
\rho_{2}\left(\Psi_{2}\right)=A\left(B+\cos ^{2} \phi_{2}\right)
$$

where

$$
\begin{aligned}
& A=2\left(1+k_{0}\right) /\left(1+k_{0}+k_{0}^{2}\right) \\
& B=k_{0}^{2}\left(2+4 k_{0}+3 k_{0}^{2}\right) / 2\left(1+k_{0}\right)^{2}\left(1+2 k_{0}\right)
\end{aligned}
$$

This serves to define the probability density of $\phi_{2}$ in the XY-plane of the twice scattered beam, $\phi_{2}$ being measured counterclockwise from the original $\mathrm{X}$-axis, and is the basis for the classical Barkla experiment. 
33. Pair production. (TAR, I=3). As a final example of collision with target at rest, we consider the case of "pair production," in which a sufficiently energetic phnton interacts with a charged particle, the transformation, of form

$$
\begin{aligned}
& A\left(e_{1}, e_{2}\right) \rightarrow S\left(e_{3}, e_{4}, e_{5}\right) \\
& e_{1}=0 \quad e_{2}>0 \quad e_{3}=e_{2} \quad e_{4}=e_{5}=e(\varepsilon)
\end{aligned}
$$

resulting in the recoil of the unexcited target, and the formation of a positron, electron pair $\varepsilon^{+}, \varepsilon^{-}$, each with rest energy $.511 \mathrm{MeV}$. The reaction is seen to have a $Q$ value

$$
Q=-2 e(\varepsilon)=-1.022 \mathrm{MeV}
$$

and consequently an energy threshold condition

$$
h v_{1} \equiv k_{1} \geq k_{T}=\frac{e_{2}+e(\varepsilon)}{e_{2}}(2 e(\varepsilon))=2 e(\varepsilon)\left\{1+\frac{e(\varepsilon)}{e_{2}}\right\}
$$

which is here sufficient as well as necessary. Note that $k_{T} \simeq 2 e(\varepsilon)$ for a nuclear target (the usual case), whereas $k_{T}=4 e(\varepsilon)$ for a target electron. We shall assume $k_{1}>k_{T}$, with a non-coherent result.

The initial system $A\left(e_{1}, e_{2}\right)$ being identical with that of 531 , all particle parameters of $A$, and of $A^{\prime}$ in the $\Sigma^{\prime}$ frame of the class, as well as the parameters of the class itself, are already given there.

The product system $S^{\prime}\left(e_{3}, e_{4}, e_{5}\right)$, of class $\left\{0, e_{0}\right\}$ in $\Sigma^{\prime}$, has a total k.e. $k_{s}^{\prime}=e_{0}-\left(e_{2}+2 e(\varepsilon)\right)>0$, where $e_{0}=\left\{e_{2}^{2}+2 e_{2} k_{1}\right\}^{\frac{3}{2}}$. The sharing of this among the three product particles is of course not unique. The target, for example, may recoil in $\Sigma^{\prime}$ with any k.e. on the range 


$$
0 \leq k_{3}^{\prime} \leq k_{j}^{\prime}=\frac{k^{\prime} s}{e_{0}}\left\{2 e(\varepsilon)+\frac{1}{2} k_{s}^{\prime}\right]
$$

as shown in Corollary 21.2. The limiting case $k_{3}=k_{j}^{\prime}$ would demand the coalesced emission of the charged pair in the direction opposite to that of the recoil target, which is physically impossible. However, their nearly parallel emission in $\Sigma$ is indeed observed. If the target were left motionless in $\Sigma^{\prime}$, with $k_{3}^{\prime}=0$, the pair would then form a two-particle system of class $\left\{0, e_{0}-e_{2}\right\}$, having the unique energies $k_{i}=k_{s} ! 2, i=4,5$, and oppositely directed momenta of equal magnitude, with $c p_{i}^{i}=\left(E_{i}^{2}-e^{2}(\varepsilon)\right)^{\frac{1}{2}}$, $E_{i}=\left(e_{0}-e_{2}\right) / 2$

34. Collisions with target in motion. Finally, we turn to collisions $A\left(e_{1}, e_{2}\right) \rightarrow S\left(e_{i}\right)$ in which both particles of a non-coherent system $A$ are in motion in $\Sigma$, with parameters

$$
k_{h}>0, E_{h}>e_{h} \geq 0, c p_{h}=\left(E_{h}^{2}-e_{h}^{2}\right)^{\frac{1}{2}}, c P_{h}=c p_{h} \Psi_{h} ; h=1,2
$$

The totals of $A$ are then

$$
e_{a}=e_{1}+e_{2} \quad k_{a}=k_{1}+k_{2} E_{0}=E_{1}+E_{2} \quad c P_{0}=c P_{1}+c P_{2}=c P_{0} \Psi_{0}
$$

We first show how all icalar parameters may be obtained in terms of the $e_{h}, E_{h}$ and the angle $\sigma$ of separation of the $\Sigma$ lines of flight:

$$
\cos \sigma=\Psi_{1} \cdot \Psi_{2} ; \quad 0 \leq \sigma \leq 180^{\circ}
$$

$$
\text { Thus } \begin{aligned}
c p_{0}^{2} & =\left|c P_{1}+c p_{2}\right|^{2}=c p_{1}^{2}+c p_{2}^{2}+2 c p_{1} c p_{2} \cos \sigma \\
& =E_{1}^{2}-e_{1}^{2}+E_{2}^{2}-e_{2}^{2}+2 c p_{1} c p_{2} \cos \sigma
\end{aligned}
$$




$$
\begin{aligned}
& =\left(E_{1}+E_{2}\right)^{2}-2_{1}^{2}-e_{2}^{2}-2\left(E_{1} E_{2}-c p_{1} c p_{2} \cos \sigma\right), \text { and hence } \\
& c p_{0}^{2}=E_{0}^{2}-\left(e_{1}^{2}+e_{2}^{2}+2 E_{\sigma}^{2}\right)
\end{aligned}
$$

where

$$
\mathrm{E}_{\sigma}^{2} \equiv \mathrm{E}_{1} \mathrm{E}_{2}-\mathrm{c} p_{1} \mathrm{c} p_{2} \cos \sigma>\mathrm{e}_{1} \mathrm{e}_{2} \geq 0
$$

It follows that the critical energy of the class is

$$
e_{0}=\left(e_{1}^{2}+e_{2}^{2}+2 E_{\sigma}^{2}\right)^{\frac{1}{2}}=\left(e_{a}^{2}+2\left(E_{\sigma}^{2}-e_{1} e_{2}\right)\right)^{\frac{1}{2}}>e_{a}
$$

and the Lorentz parameters are as usual

$$
\gamma_{0}=E_{0} / e_{0} \quad \beta_{0}=c p_{0} / E_{0} \quad \Psi_{0}=c P_{0} / c p_{0}
$$

From (6) we see that the threshold condition $e_{0} \geq e_{s}$ for the proposed transmutation here takes the form

$$
\mathrm{E}_{\sigma}^{2} \geq \mathrm{e}_{1} \mathrm{e}_{2}+\frac{\mathrm{e}_{\mathrm{a}}+\mathrm{e}_{\mathrm{s}}}{2}(-\mathrm{Q})
$$

where $Q=e_{a}-e_{s}$ is the Q-value of the reaction. We shall assume $e_{o}>e_{s}$ and $I \geq 2$, so that a non-coherent result $S\left(e_{i}\right)$ is possible.

If the collision $A^{\prime}\left(e_{1}, e_{2}\right) \rightarrow S^{\prime}\left(e_{i}\right)$ is to be studied in the $\Sigma^{\prime}$ frame of the class, we may require the (unique) parameters

$$
E_{h}^{\prime}=\left(e_{h}^{2}+E_{\sigma}^{2}\right) / e_{o}, \quad c p_{h}^{\prime}=\left(E_{\sigma}^{4}-e_{1}^{2} e_{2}^{2}\right)^{\frac{1}{2}} / e_{o} \quad h=1,2
$$


The angles $\bar{\psi}_{1}, \bar{\psi}_{1}^{\prime}$ which $\mathrm{CP}_{1}$ and $\mathrm{CP}_{1}^{\prime}$ make with the direction $\Psi_{0}$ of the class velocity may also be needed. The first of these is obtained from

$$
\begin{aligned}
\cos \tilde{\psi}_{1} & =\mathrm{cP}_{0} \cdot \mathrm{cP}{ }_{1} / c p_{0} c p_{1}=\left(c P_{1}+c P_{2}\right) c P_{1} / c p_{0} c p_{1} \\
& =\left\{\left(c p_{1}\right)^{2}+c p_{1} c p_{2} \cos \sigma\right\} / c p_{0} c p_{1}
\end{aligned}
$$

Using this, we may find $\cos \bar{\psi}_{1}$ from the Lorentz transformation:

$\overline{c p}_{1 \mathrm{x}}=\gamma_{\mathrm{o}}\left(\overline{c p}_{1 \mathrm{x}}-\bar{B}_{\mathrm{o}} \mathrm{E}_{1}\right)=\left(\mathrm{E}_{\mathrm{o}} c p_{1} \cos \bar{\psi}_{1}-c p_{0} \mathrm{E}_{1}\right) / \mathrm{e}_{0}$, giving $c p_{0} e_{0} c p_{1}^{\prime} \cos \bar{\psi}_{1}^{\prime}=$ $E_{0}\left(c p_{0} c p_{1} \cos \bar{\psi}_{1}\right)-\left(c p_{0}\right)^{2} E_{1}$. Hence by $(10), c p_{0}\left(e_{0} c p_{1}^{1}\right) \cos \Psi_{1}=$ $E_{0}\left\{\left(c p_{1}\right)^{2}+c p_{1} c p_{2} \cos \sigma\right\}-\left(E_{0}^{2}-e_{o}^{2}\right) E_{1}=E_{o}\left(E_{1}^{2}-e_{1}^{2}\right)+E_{o}\left(E_{1} E_{2}-E_{\sigma}^{2}\right)-E_{o}^{2} E_{1}+$ $\left(e_{1}^{2}+e_{2}^{2}+2 \varepsilon_{\sigma}^{2}\right) E_{1}=e_{2}^{2} E_{1}+e_{1}^{2}\left(E_{1}-E_{0}\right)+E_{0} E_{1}\left(E_{1}+E_{2}-E_{0}\right)+E_{\sigma}^{2}\left(-E_{0}+2 E_{1}\right)$ $=e_{2}^{2} E_{1}-e_{1}^{2} E_{2}+E_{\sigma}^{2}\left(E_{1}-E_{2}\right)$

Hence from this and (9) we obtain

$$
\cos \bar{\psi}_{1}=\left\{e_{2}^{2} E_{1}-e_{1}^{2} E_{2}+E_{\sigma}^{2}\left(E_{1}-E_{2}\right)\right\} / c p_{0}\left(E_{\sigma}^{4}-e_{1}^{2} e_{2}^{2}\right)^{\frac{1}{2}}
$$

Some procedures are given below for various kinds of collision with target (2) in motion, as well as projectile (1). 
Method I. Given $e_{h}, E_{h}, c_{h}$ on $\Sigma$ axes $A$; collision treated in $\Sigma^{\prime}$ frame of class; $\Psi_{1}$ specified as basic direction for non-polarized emission in $\Sigma$ ', auxiliary $\Sigma^{\prime}$ direction $\Omega^{\prime}$ obtained by sampling (App. II).

1. $\mathrm{CP}_{0}=\mathrm{cP} \mathrm{P}_{1}+\mathrm{cP} \mathrm{P}_{2}$ on $\mathrm{A}, \mathrm{CP} \mathrm{P}_{\mathrm{o}}=\left|\mathrm{cP} \mathrm{o}_{0}\right|$

2. $\Psi_{0}=c P_{0} / C p_{0}$ class direction on $A$.

3. $e_{0}=\left(E_{0}^{2}-c p_{0}^{2}\right)^{\frac{1}{2}}$. Test $e_{0}>e_{s}$.

4. $\left(\mathrm{CP}_{1}\right)_{S}=\mathrm{D}^{\tau}\left(\mathrm{CP}_{1}\right)_{A}$ gives components of $\mathrm{CP}_{i}$ on standard $\Sigma$ axes $S$, where $D$ is matrix of App. I, Corollary 1 based on rotation $\delta$ taking $X$ into $\Psi_{0}=\left(a_{o x}, a_{o y}, a_{o z}\right)_{A}$ of step 2 .

5. $\left(c P_{I}^{\prime}\right)_{S^{\prime}}=\left[\begin{array}{l}\left(E_{0} \overline{c p}_{1 x}-c p_{0} E_{1}\right) / e_{0} \\ \overline{c p}_{1 y} \\ \overline{c p_{1 z}}\end{array}\right]$

gives $S^{\prime}$ components of $\mathrm{cP}_{1}^{\prime}$ in $\Sigma^{\prime}$.

6. $\left(\Psi_{1}\right)_{S^{\prime}}=\left(\mathrm{CP}_{1}^{\prime}\right)_{S^{\prime}} / \mathrm{CP}_{1}^{\prime}$ is the basic direction for emission in $\Sigma^{\prime}$, where $c p_{1}^{\prime}=\left|\left(\mathrm{cP}_{1}^{\prime}\right)_{S},\right|$.

7. If collision is elastic, set $c p_{3}^{\prime}=c p_{1}^{\prime}, E_{3}^{\prime}=E_{1}^{\prime}$ where $E_{1}^{\prime}=$ $\left(-c p_{0} \overline{C p}_{1 x}+E_{0} E_{1}\right) / e_{0}$. In any case, we must suppose $E_{3}^{\prime}, c p_{3}^{\prime}=$ $\left(E_{3}^{2}-e_{3}^{2}\right)^{\frac{1}{2}}$, and auxiliary direction $\Omega^{\prime}$ of the particle (3) to be followed as physically determined in $\Sigma^{\prime}$ at this point.

8. The direction of 3 on $\Sigma^{\prime}$ axes $S^{\prime}$ is given by

$$
\left(\Psi_{3}^{\prime}\right)_{S^{\prime}}=\tilde{D}\left(\Omega^{\prime}\right)_{S^{\prime}}
$$


where $\tilde{D}$ is no: the matrix D of App. I, Cor. 1 based on rotation $\delta$ taking $\bar{X}^{\prime}$ into $\Psi_{i}=\left(a_{1 x}, a_{1 y}, a_{1 z}\right)_{S}$, from step 6.

9. $\left(\mathrm{CP}_{3}^{\prime}\right)_{S^{\prime}}=\mathrm{CP}_{3}^{\prime}\left(\Psi_{3}^{\prime}\right)_{S^{\prime}}$, gives the momentum of 3 on $\Sigma^{\prime}$ axes $S^{\circ}$.

10. $\overline{c p}_{3 x}=\left(E_{0} \overline{c p}_{3 x}^{\prime}+c p_{0} E_{3}^{1}\right) / e_{0}$

$\bar{c}_{3 y}=\overline{c p}_{3 y}^{\prime}$

$\overline{C P}_{3 z}=\overline{c p}_{3 z}$

$E_{3}=\left(c p_{0} \overline{c p}_{3 x}^{\prime}+E_{0} E_{3}^{\prime}\right) / e_{0}$

gives $\mathrm{E}_{3}$, and $\left(\mathrm{CP}_{3}\right)_{S}$ on standard $\sum$ axes $S$.

11. Finally $\left(\mathrm{cP}_{3}\right)_{A}=\mathrm{D}\left(\mathrm{CP}_{3}\right)_{S}$ gives the momentum of 3 on original $\sum$ axes $A$, where $D$ is the matrix of step 4 . The ideas are schematized in Fig. 1.

Method II. (Input as in Method 1.) This is a modification of Method I. Steps 1-3 are the same.

4. $\Psi_{1}=c P_{1} / c p_{1}$ is direction of 1 on $\sum$ axes $A$. One now computes matrix $D_{2}$ (App. I, Th. 3) based on rotation $\delta_{2}$ which not only takes $X$ into $\Psi_{0}$ but has $\Psi_{1}$ in the upper half of $\delta_{2} X, \delta_{2} Y$ plane. Standard axes $S=(\bar{X}, \bar{Y}, \bar{Z})$ are thus obtained with the parallelogram of Fig. 1 contained in the $\bar{X}, \bar{Y}$ plane. The matrix $D_{2}$ is not used until the final step.

5. $C=\cos \bar{\psi}_{1}^{\prime}$ is computed from Eq. (11), and we set $s=+\left(1-c^{2}\right)^{\frac{1}{2}}$.

6. The basic direction on $\Sigma^{\prime}$ axes $S^{\prime}$ is then $\left(\Psi_{1}^{\prime}\right)_{S^{\prime}}=(C, S, 0)_{S^{\prime}}$.

7. If collision is elastic, set $c p_{3}^{\prime}=c p_{1}^{\prime}, E_{3}^{\prime}=E_{1}^{\prime}$ where now $c p_{1}^{\prime}$ and $E_{1}$ are computed from Eq. (9). Otherwise as before in step 7. 


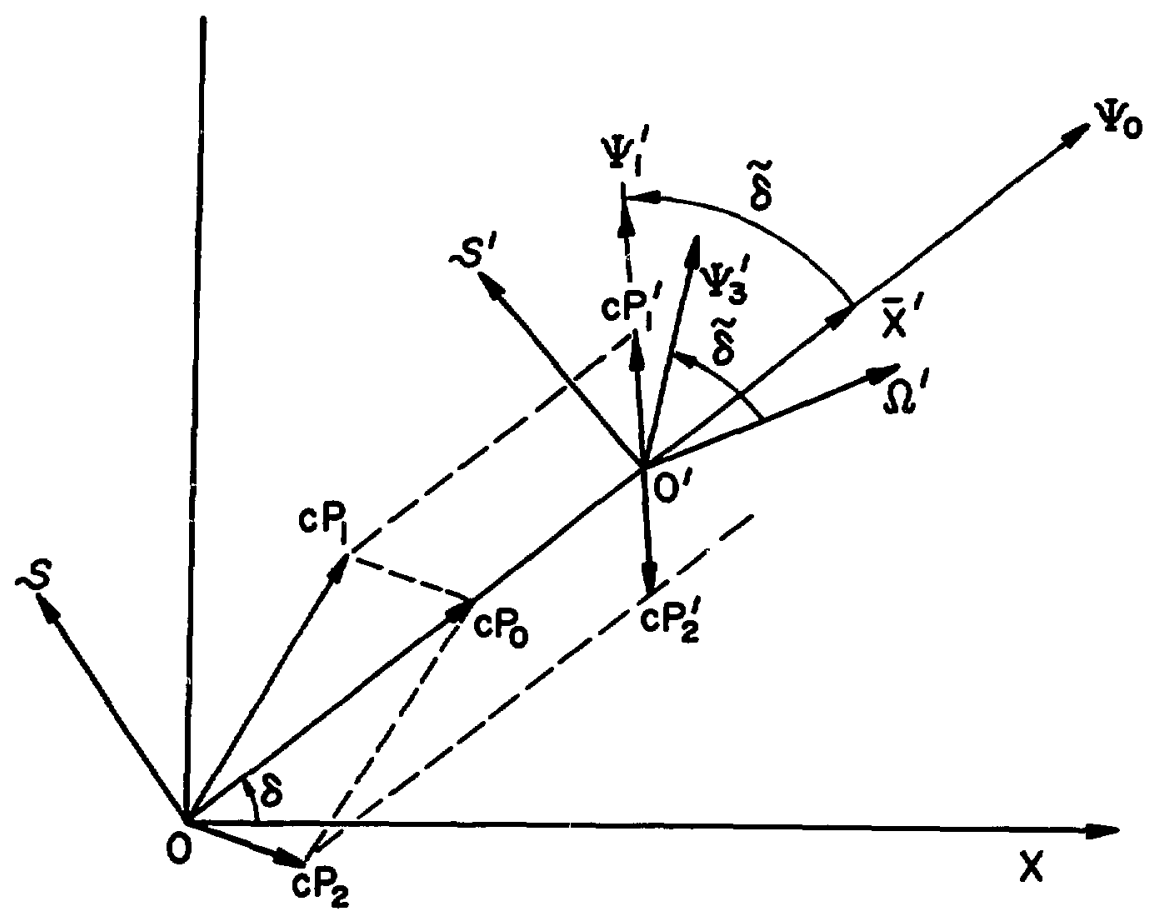

Fig. 1.

Idea of Method I. 
8. The direction of 3 on $\Sigma$ ' axes $S \cdot$ is now

$$
\begin{aligned}
& \left(\Psi_{3}^{\prime}\right)_{S},=\tilde{D}\left(\Omega^{\prime}\right)_{S}, \\
& \text { where } \tilde{D}=\left[\begin{array}{rrr}
C & -S & 0 \\
S & C & 0 \\
0 & 0 & 1
\end{array}\right] \text { is the matrix based on rotation of taking } \bar{X}^{\prime} \\
& \text { into }\left(\Psi_{1}^{\prime}\right)_{S} \cdot \cdot
\end{aligned}
$$

Steps 9-11 are the same, except that one uses the matrix $D_{2}$ in place of $D$ in step 11.

Method III. In one type of problem, the parameters $e_{1}, E_{1},\left(\mathrm{CP}_{1}\right)_{A}$ of the projectile are given, but the nature of the target is subject to chance. Suppose $e_{2}, E_{2}, C P_{2}$ suitably chosen, and that the direction $\Psi_{2}$ is in a non-polarized distribution about $\Psi_{1}$. Finally, suppose the (latitude) angle $\sigma$ of separation, $0^{\circ} \leq \sigma \leq 180^{\circ}$, and a longitude $\phi, 0^{\circ} \leq \phi<360^{\circ}$, chosen for location of $\Psi_{2}$ about the "basic direction" $\Psi_{1}$.

One may of course proceed by Methods I or II if one first specifies $\left(\Psi_{2}\right)_{A}$. This may be done (App. II) by defining $(\Omega)_{A}=(\cos \sigma, \sin \sigma \cos \phi$, $\sin \sigma \sin \phi)_{A}$ and setting

$$
\left(\Psi_{2}\right)_{A}=D(\Omega)_{A}
$$

where $D$ is the matrix of the rotation taking $X$ into $\Psi_{1}$ (App. $I$, Cor. 1).

There is another strategy available, outlined in LAMS-2360 (Metropolis, Turkevich, et al.) and slightly modified here. (See Fig. 2.)

1. We first define $\Sigma$ axes

$$
A_{1}=\left[\delta_{1} X, \delta_{1} Y, \delta_{1} Z\right]=[X, Y, Z] D_{1}
$$




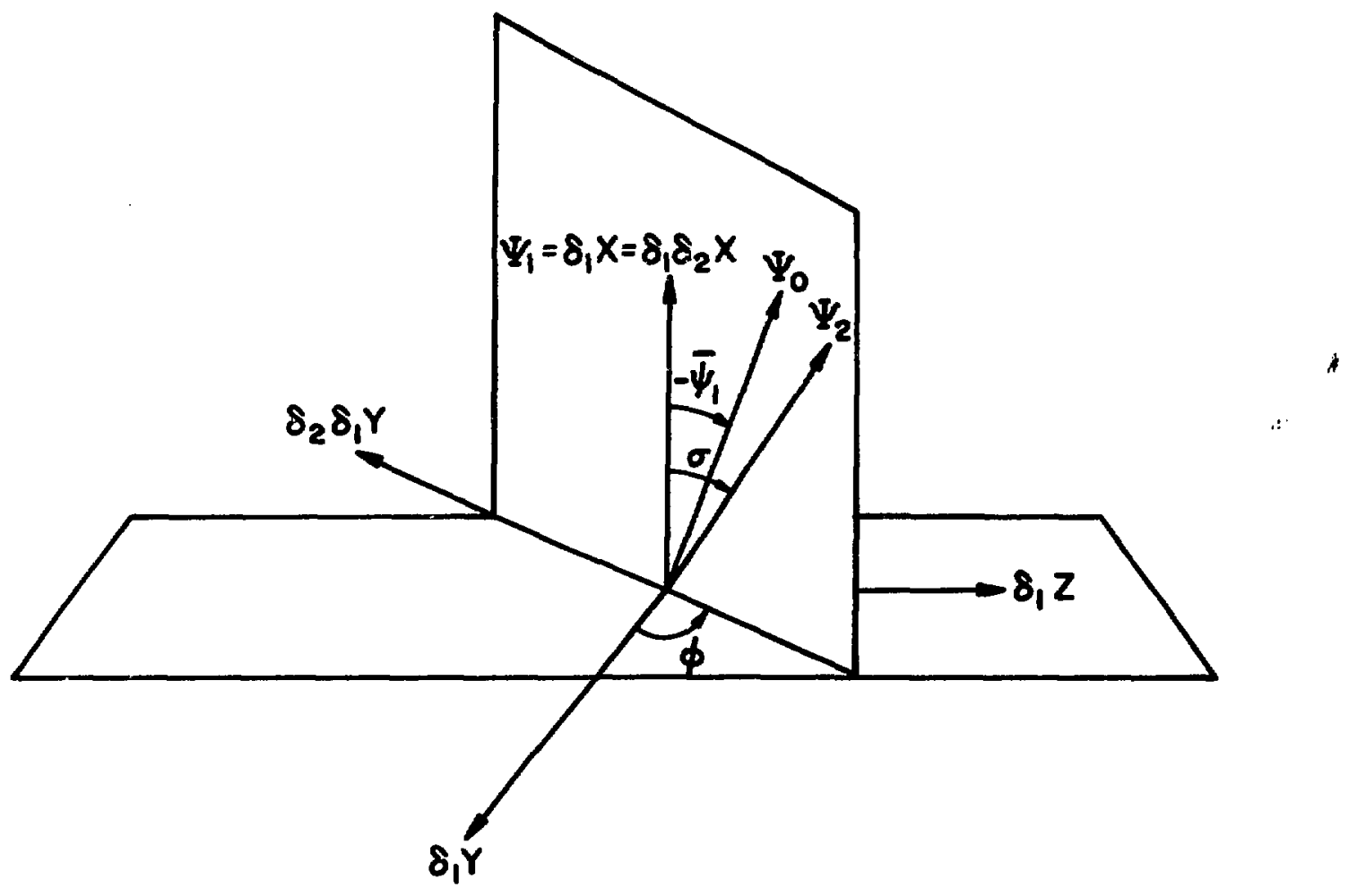

Fig. 2.

Idea of Method III. 
where $D_{1}$ is the matrix based on the rotation $\delta_{1}$ taking $X$ into $\Psi_{1}$ (App.. I, Cor. 1)

2. We now specify $\Psi_{2}$ on the axes $A_{1}$ by $\left(\Psi_{2}\right)_{A_{1}}=(\cos \sigma, \sin \sigma$ $\cos \phi, \sin \sigma \sin \phi)_{A_{1}}$, but make no use of this.

3. The rotation $\delta_{2}$, about $\Psi_{1}$, through $\phi+180^{\circ}$ is defined relative to the axes $A_{1}$ by

$$
A_{2}=\delta_{2}\left[\delta_{1} X, \delta_{1} Y, \delta_{1} Z\right]=\left[\delta_{1} X, \delta_{1} Y, \delta_{1} Z\right] D_{2}
$$

where

$$
D_{2}=\left[\begin{array}{ccc}
1 & 0 & 0 \\
0 & \cos \left(\phi-180^{\circ}\right) & -\sin \left(\phi+180^{\circ}\right) \\
0 & \sin \left(\phi+180^{\circ}\right) & \cos \left(\phi+180^{\circ}\right)
\end{array}\right]
$$

The directions $\Psi_{1}, \Psi_{0}$, and $\Psi_{2}$ now appear in the $\delta_{2} \delta_{1} x, \delta_{2} \delta_{1} Y$ plane with $\Psi_{1}=\delta_{2} \delta_{1} \mathrm{X}$, and $\Psi_{0}, \Psi_{2}$ in its lower half. (Fig. 2.)

4. The cos $\Psi_{1}$ between $\mathrm{CP}_{0}$ and $\mathrm{CP}_{1}$ is now computed from Eq. (10), and used to obtain the matrix

$$
D_{3}=\left[\begin{array}{ccc}
\cos \left(-\bar{\psi}_{1}\right) & -\sin \left(-\bar{\psi}_{1}\right) & 0 \\
\sin \left(-\bar{\psi}_{1}\right) & \cos \left(-\bar{\psi}_{1}\right) & 0 \\
0 & 0 & 1
\end{array}\right]
$$

of the rotation $\delta_{3}$, about $\delta_{2} \delta_{1} z$, through $-\psi_{1}$, which determines the final standard $\Sigma$ axes

$$
S=[\bar{X} \bar{Y} \bar{Z}]=\delta_{3}\left[\delta_{1} \delta_{2} X, \delta_{1} \delta_{2} Y, \delta_{1} \delta_{2} Z\right]=\left[\delta_{1} \delta_{2} X, \delta_{1} \delta_{2} Y, \delta_{1} \delta_{2} Z\right] \cdot D_{3}
$$


The situation is now precisely that of Method II, with $\bar{X}=\Psi_{0}$, and $\Psi_{1}$ in the upper half of the $\bar{X}, \bar{Y}$ plane. Note that no computing has yet been done except for evaluation of the three matrices $D_{i}$.

It will be necessary on the return trip to know the matrix $D_{4}$ defined by

$$
\delta_{4}[X, Y, Z]=[X, Y, Z] D_{4}
$$

where $\delta_{4}$ is the composite rotation $\delta_{3} \delta_{2} \delta_{1}$ and $[X, Y, Z]$ are the original $\Sigma$ axes $A$. Retracing our steps, we see that

$$
\begin{aligned}
S=\delta_{4} A & =\delta_{3}\left(\delta_{2} \delta_{1} A\right)=\left(\delta_{2} \delta_{1} A\right) D_{3}=\delta_{2}\left(\delta_{1} A\right) D_{3} \\
& =\left(\delta_{1} A\right) D_{2} D_{3}=A D_{1} D_{2} D_{3}=A D_{4}
\end{aligned}
$$

so we now compute the matrix

$$
D_{4}=D_{1} D_{2} D_{3}
$$

5. We next obtain $C=\cos \bar{\psi}_{1}^{\prime}$ from Eq. $(11)$, and set $s=\left(1-C^{2}\right)^{\frac{1}{2}}$.

6. The basic direction in $\Sigma^{\prime}$ is then $\left(\Psi_{1}^{\prime}\right)_{S^{\prime}}=(C, S, 0)_{S^{\prime}}$ on standard ¿' axes $S^{\prime}$ parallel to $S$.

7. If collision is elastic, set $c p_{3}^{\prime}=c p_{1}^{\prime}, E_{3}^{\prime}=E_{1}^{\prime}$, where $c p_{1}^{\prime}, E_{1}^{\prime}$ are computed from $\mathrm{Eq}$. (9). In any case, we assume $E_{3}^{\prime}, C P_{3}^{:}$and auxiliary direction $\left(\Omega^{\prime}\right)_{S}$ ' given physically.

8. Direction of 3 on $\Sigma^{\prime}$ axes $S^{\prime}$ is

$$
\left(\Psi_{3}^{\prime}\right)_{S^{\prime}}=\tilde{D}\left(\Omega^{\prime}\right)_{S^{\prime}}
$$


where

$$
Z=\left[\begin{array}{rrr}
C & -S & 0 \\
S & C & 0 \\
0 & 0 & 1
\end{array}\right]
$$

9-11 are the same as in Method I, except that in step 11 we compute $\left(\mathrm{CP}_{3}\right)_{A}=\mathrm{D}_{4}\left(\mathrm{CP}_{3}\right)_{S}$ where $\mathrm{D}_{4}=\mathrm{D}_{1} \mathrm{D}_{2} \mathrm{D}_{3}$ is the matrix of step 4 above.

Method IV. For problems with the input of Method I, there is always the alternative of the vector form of the cPE (10.5) transformation, avoiding the rotation to standard axes.

1. One computes $\mathrm{cP}_{0},\left(\Psi_{0}\right)_{A}, e_{0}$ as in Method $I$, and sets

$$
\gamma_{0}=E_{0} / e_{0}, \quad B_{0}=c p_{0} / E_{0}
$$

Then $\left(\mathrm{cP}_{1}\right)_{A^{\prime}}=\left(\mathrm{cP}_{1}\right)_{A}+\left\{\delta_{0}\left(\mathrm{cP}_{1} \cdot \Psi_{0}\right)-\gamma_{0} B_{0} E_{1}\right\}\left(\Psi_{0}\right)_{A}$

$$
E_{1}^{\prime}=\gamma_{0}\left\{-\beta_{0}\left(c P_{1} \cdot \Psi_{0}\right)+E_{1}\right\}
$$

and $\left(\Psi_{1}\right)_{A^{\prime}}=\left(C P_{1}^{\prime}\right)_{A^{\prime}} / c P_{1}^{\prime}$ is the basic direction on parallel axes $A^{\prime}$.

2. The rotation $\delta$, with matrix $D$ based on the latter unit vector, takes $X^{\prime}$ into $\Psi_{1}^{\prime}$, and hence

$$
\left(\Psi_{3}^{\prime}\right)_{A^{\prime}}=D\left(\Omega^{\prime}\right)_{A^{\prime}}
$$

(App. I, Cor. 1)

3. From stipulated $\mathrm{E}_{3}^{\prime}$ and $\mathrm{CP} \mathrm{P}_{3}^{\prime}=c \mathrm{P}_{3}^{\prime}\left(\Psi_{3}^{\prime}\right)_{A}$, the direct transformation then yields 


$$
E_{3}=\gamma_{0}\left\{\beta_{0}\left(C P P_{3} \cdot \Psi_{0}\right)+E_{3}^{\prime}\right\}
$$

and

$$
\left(\mathrm{CP}_{3}\right)_{A}=\left(\mathrm{CP}_{3}^{\prime}\right)_{A^{\prime}}+\left\{\delta_{0}\left(\mathrm{CP} P_{3}^{\cdot} \cdot \Psi_{0}\right)+\gamma_{0} B_{0} E_{3}^{\prime}\right\}\left(\Psi_{0}\right)_{A}
$$

on the original $\Sigma$ axes $A$. This method requires a minimum of "formulas" and may well surpass the others in speed.

Method V. Assumptions: $e_{h}, E_{h},{ }^{c} P_{h}$ given on $\Sigma$ axes $A$; collision treated in rest frame $\Sigma '$ of the targer $\left(e_{2}>0\right) ; \Psi_{1}^{\prime}$ specified as basic direction for non-polarized emission in $\Sigma^{\prime} ; \theta^{\prime}, \phi^{\prime}$ chosen for location of $\Psi_{3}^{\prime}$ about $\Psi_{1}^{\prime}$. The method is indicated when differential cross sections are given in the rest frame of the target, as in Compton scattering, which has already been considered. The appropriate Lorentz transformations between $\Sigma$ and $\Sigma$ ' now have the parameters of the target in $\Sigma$, namely

$$
\gamma_{0}=\gamma_{2}=E_{2} / e_{2} \quad \beta_{0}=\beta_{2}=c p_{2} / E_{2} \quad \Psi_{0}=\Psi_{2}=c P_{2} / c p_{2} .
$$

The procedure is that of Methods I or IV, with obvious modifications, the target rest frame playing the role of the $\Sigma^{\prime}$ frame of the class. The emission parameters must of course be stipulated in $\Sigma '$. Note that the formulas (9-11) are no longer valid.

Note 1. Figure 1 is only schematic. The true relations between the systems A, A' may be seen as usual from a suitable ellipsoid figure based on sphere radii

$$
c p_{h}^{\prime}, \gamma_{0} c p_{h}^{\prime} \text {, and distances } 0_{h}^{\prime 0}=\gamma_{0} \beta_{0} E_{h}^{\prime} \cdot \text { (Fig. 20.1) }
$$

In such a figure, the three cases for (1) are here distinguished by the inequalities 


$$
c p_{1} c p_{2} \cos \sigma \stackrel{\risingdotseq}{\lessgtr}\left(E_{2}-e_{1}\right) k_{1}
$$

and for (2) by

$$
c p_{1} c p_{2} \cos \sigma \leqq\left(E_{1}-e_{2}\right) k_{2}
$$

This is easily proved from the equivalent form $\gamma_{0} e_{h} \sum_{h}^{\prime}$ of Note 13.3, using $\gamma_{0}=E_{0} / e_{0}$, and the values of $E_{1}^{1}, E_{\sigma}^{2}$ from Eqs. (9) and (5).

Note 2. We have tacitly assumed in this section that $\mathrm{CP}_{0} \neq 0$, with a welldefined direction $\Psi_{0^{\circ}}$. This indeed fails in the single case $\sigma=180^{\circ}$, $c p_{1}=c p_{2}$. The frame $\Sigma$ is then identical with the $\Sigma^{\prime}$-frame of the class, and one by-passes the Lorentz transformation from $\Sigma$ to $\Sigma^{\prime}$.

Note 3. The inequality in (5) is obvious from (6), A being non-coherent. 35. Colliding beams. For a collision

$$
A\left(e_{1}, e_{2}\right) \rightarrow S\left(e_{i}\right)
$$

between two particles of equal rest energy $e_{1}=e_{2}=e$, and fixed energies $E_{1}, E_{2}$ in the lab. frame $\Sigma$, the total energy in the $\Sigma$ ' frame of the class is given by $(34.6)$ as

$$
e_{0}=\left(2 e^{2}+2 E_{1} E_{2}-2 c p_{1} c p_{2} \cos \sigma\right)^{\frac{1}{2}}
$$

This energy, which is critical for the production of new particles, as witnessed by the necessary condition

$$
e_{0} \geq e_{s}
$$

naturally reaches its maximum value

$$
e_{0}\left(E_{1}, E_{2}\right)=\left(2 e^{2}+2 E_{1} E_{2}+2 c p_{1} c p_{2}\right)^{\frac{1}{2}}
$$


in the case of a "head-on" collision, with $\sigma=180^{\circ}$.

Now suppose $E_{1}>e_{1}$ is fixed, say at the greatest energy to which such a particle can be accelerated in $\Sigma$ by present methods. Then, if the target energy varies from $E_{2}=e$ (limit case, target at rest) to $E_{2}=E_{1}$ (as in two optimal colliding beams), this $\Sigma^{\prime}$ energy rises from

$$
e_{0}\left(E_{1}, e\right)=\left(2 e^{2}+2 e E_{1}\right)^{\frac{1}{2}} \quad(T A R)
$$

to its maximal value

$$
e_{0}\left(E_{1}, E_{1}\right)=\left(2 e^{2}+2 E_{1}^{2}+2\left(E_{1}^{2}-e^{2}\right)\right)^{\frac{1}{2}}=2 E_{1} \quad \text { (C. B. ) }
$$

In the latter case, the oppositely directed momenta are of equal magnitude, and the frames $\Sigma, \Sigma^{\prime}$ coincide.

Thus a factor $e_{0}\left(E_{1}, E_{1}\right) / e_{0}\left(E_{1}, e\right)=\sqrt{ } 2 \gamma_{1} /\left(\gamma_{1}+1\right)^{\frac{1}{2}}>1$ is gained in the critical energy $e_{0}$. To appreciate this rather modest looking factor, one must ask what $\Sigma$ projectile energy $E_{1}^{*}$ would be required for collision on target at rest to achieve the same energy $2 \mathrm{E}_{1}$ in $\Sigma^{\prime}$. The answer is obviously provided by the equation

$$
\left(2 \mathrm{e}^{2}+2 \mathrm{eE}_{1}^{*}\right)^{\frac{1}{2}}=2 \mathrm{E}_{1}
$$

name1y,

$$
E_{1}^{*}=\left(2 E_{1}^{2}-e^{2}\right) / e
$$

Measuring energy in units of the rest energy e, one may verify the following table 


$\begin{array}{rrrr} & \text { TAR } & \text { C.B. } & \text { TAR } \\ E_{1} & e_{0}\left(E_{1}, e\right) & 2 E_{1} & E_{1}^{*} \\ 10 & 4.7 & 20 & 199 \\ 25 & 7.2 & 50 & 1249 \\ 50 & 10.1 & 100 & 4999 \\ 100 & 14.2 & 200 & 19999 \\ & & & 2 E_{1}^{2}-1\end{array}$




\section{Chapter IV}

\section{CROSS SECTIONS}

36. Mean free path in a gas. If in traversing a distance $d \ell$ in $\Sigma$ through a medium of total numerical density $n$ particles $/ \mathrm{cm}^{3}$, a projectile 1 of k.e. $k_{1}>0$ has probability nsdl of collision, we call $s=s\left(k_{1}\right)$ the effective cross section of the medium. This assumption is equivalent to the differential equation $\mathrm{dT} / \mathrm{T}=-n s d \ell$ for the probability $\mathrm{T}(\ell)$ of transmission without collision through distance $\ell$, i.e.,

$$
\mathrm{T}(\ell)=\mathrm{e}^{-n s \ell}
$$

Hence $P(\ell)=1-T(\ell)=1-e^{-n s \ell}$ is the probability distribution function for first collision at distance $\leq \ell$, and

$$
\mathrm{p}(\ell)=\mathrm{p}^{\prime}(\ell)=\text { nse } e^{-n s l}
$$

is the corresponding probability density for first collision on $(\ell, \ell+d \ell)$. The "mean free path" is therefore

$$
L=\ell=\int_{0}^{\infty} \ell p(\ell) d \ell=1 / n s
$$

In Monte Carlo practice, a random number $r$, uniformly distributed on $(0,1)$ determines the distance $\ell$ to first collision by means of the formula $\mathbf{r}=P(\ell), i \cdot e$,

$$
\ell=-L \ln (1-r)
$$

In the case of a composite medium, with $n_{i}=n f_{i}$ particles of cype $i$, the probability of collision on $d e$ is

$$
\sum n_{i} s_{i} d l=n\left(\sum f_{i} s_{i}\right) d l
$$


where $s_{i}$ is the cross section of type $i$, and hence

$$
\Delta=\sum \mathbf{f}_{\mathbf{i}}:{ }_{\mathbf{i}}
$$

is the effective cross section of the composite medium.

In the following, an attempt is made to obtain an effective cross section for a pure gas in terms of its k.e. distribution, and its cross section $s^{r}\left(k_{1}^{\prime}\right)$ obtaining for a projectile of $k_{. e} \cdot k_{1}^{\prime}$ traversing the gas at rest.

As a preliminary step, consider a projectile 1 of proper energy $e_{1}$, k.e. $k_{1}>0$, direction $\Psi_{1}=(1,0,0)$ traversing distance $d \ell$ through a medium of $\mathrm{n}_{i}$ identical particles 2 per $\mathrm{cm}^{3}$, all with energy $E_{2}$ and direction $\Psi_{2}$. In the common rest frame of the targets, the projectile has energies (Note 10.1)

$$
E_{1}^{\prime}=\left(E_{1} E_{2}-c p_{1} c p_{2} \cos \sigma\right) / e_{2} \equiv E_{\sigma}^{2} / e_{2}, \quad k_{1}^{\prime}=E_{1}^{\prime}-e_{1}
$$

Here, $E_{\sigma}^{2}$ is the abbreviation used in 534 , with $\cos \sigma=\Psi_{1} \bullet \Psi_{2}$. The corresponding momentum magnitude is given by

$$
c P_{1}^{\prime}=\left(E_{1}^{2}-e_{1}^{2}\right)^{\frac{1}{2}}=\left(E_{\sigma}^{4}-e_{1}^{2} e_{2}^{2}\right)^{\frac{1}{2}} / e_{2}
$$

The projectile undergoes a corresponding displacement (Note 13.2)

$$
d \ell^{\prime}=d \ell c p_{1}^{\prime} / c p_{1}
$$

through a medium at rest in $\Sigma^{\prime}$, with density $(7.3)$

$$
n !=n_{i} / \gamma_{2}, \quad \gamma_{2}=E_{2} / e_{2}
$$


We might therefore expect a probability of collision

$$
n ! s^{r}\left(k_{1}^{\prime}\right) d \ell^{\prime}
$$

on $d l^{\prime}$ in $\Sigma^{\prime}$, and hence on $d l$ in $\Sigma$. Substitution of (2), (3), (4) in (5) yields

$$
n_{i}\left\{s^{r}\left(k_{1}^{\prime}\right)\left(E_{\sigma}^{4}-e_{1}^{2} e_{2}^{2}\right)^{\frac{1}{2}} / E_{2} c p_{1}\right\} d l
$$

where we shall regard

$$
s\left(k_{1} ; k_{2}, \sigma\right)=\left\{s^{r}\left(k_{1}^{\prime}\right)\left(E_{\sigma}^{4}-e_{1}^{2} e_{2}^{2}\right)^{\frac{1}{2}} / E_{2} c p_{1}\right\}
$$

as the cross section contributed by the given medium.

Now consider the traversal of the projectile through distance $d \ell$ of an isotropic gas of $n$ particles/cm ${ }^{3}$, in a k.e. "distribution" $f\left(k_{2}\right) d k_{2}$. There are then a fraction

$$
f_{i}=f\left(k_{2}\right) d k_{2} d \Psi_{2} / 4 \pi
$$

of particles on $\left(\mathrm{k}_{2}, \mathrm{k}_{2}+\mathrm{dk} \mathrm{k}_{2}\right),\left(\Psi_{2}, \Psi_{2}+\mathrm{d} \Psi_{2}\right)$, which we regard as a submedium contributing the cross section of (7). We should therefore expect in the limit an effective cross section

$$
s=s\left(k_{1}\right)=\int S \Delta\left(k_{1} ; k_{2}, \sigma\right) f\left(k_{2}\right) d k_{2} d \Psi_{2} / 4 \pi
$$


for the gas, and hence a free path $L=1 /$ ns determining distance to first collision.

Adopting spherical coordinates $\sigma, \phi$ for location of $\Psi_{2}$ about the projectile direction $\Psi_{1}$, and setting $a=\cos \sigma$, we may write (8) in the form

$$
s=s\left(k_{1}\right)=\left(2 c p_{1}\right)^{-1} \int_{0}^{\infty} \int_{-1}^{1} s^{r}\left(k_{1}^{\prime}\right)\left(E_{\sigma}^{4}-e_{1}^{2} e_{2}^{2}\right)^{\frac{1}{2}} E_{2}^{-1} f\left(k_{2}\right) d k_{2} d a
$$

The involved dependence of the integrand on the variables $k_{2}$, a cf integration is provided by the relations

$$
\begin{aligned}
& k_{1}^{\prime}=E_{1}^{\prime}-e_{1} \\
& E_{1}^{\prime}=E_{\sigma}^{2} / e_{2} \\
& E_{\sigma}^{2}=E_{1} E_{2}-c p_{1} c p_{2} a \\
& E_{2}=k_{2}+e_{2} \\
& c p_{2}=\left(E_{2}^{2}-e_{2}^{2}\right)^{\frac{1}{2}}
\end{aligned}
$$

where $E_{1}=k_{1}+e_{1}$ and $c p_{1}=\left(E_{1}^{2}-e_{1}^{2}\right)^{\frac{1}{2}}$ are constants of the projectile. Note 1. A non-relativistic analogue of (9), which is "well known" reads

$$
\begin{aligned}
s\left(k_{1}\right) & =v_{1}^{-1} s s^{r}\left(k_{1}^{\prime}\right)\left|v_{1}-v_{2}\right| F_{2}\left(v_{2}\right) d v_{2} \\
& =\left(2 v_{1}\right)^{-1} \int_{0}^{\infty} \int_{-1}^{1} s^{r}\left(k_{1}^{\prime}\right) v_{1}^{\prime} f\left(v_{2}\right) d v_{2} d a
\end{aligned}
$$

where $v_{1}^{\prime}=\left(v_{1}^{2}+v_{2}^{2}-2 v_{1} v_{2}^{a}\right)^{\frac{3}{2}}$. In case $s^{r}\left(k_{1}^{\prime}\right)=s^{r}$ is constant, one can show that $s\left(k_{1}\right)>s^{r}$ for an arbitrary distribution $f\left(v_{2}\right)$. This answers the question 
(C. Mark) whether it is easier to cross Times Square blindfolded with traffic in motion or at rest.

For a Maxwell distribution

$$
\begin{aligned}
& f\left(v_{2}\right)=\left(4 \beta_{2}^{3} ! \sqrt{ }\right) v_{2}^{2} \exp \left(-\beta_{2}^{2} v_{2}^{2}\right) \text {, with } \\
& \beta_{2}=\left(m_{2} / 2 K T\right)^{\frac{1}{2}} \text {, one finds } \\
& s\left(k_{1}\right)=s^{r}\left\{(1 / \omega / \pi) \exp \left(-\omega^{2}\right)+\left[1+\left(1 / 2 \omega^{2}\right)\right] \operatorname{Erf}(\omega)\right\}
\end{aligned}
$$

where

$$
\omega=\beta_{2} v_{1}, \quad \operatorname{Erf}(\omega) \equiv(2 / \sqrt{ } \pi) \int_{0}^{\omega} \exp \left(-x^{2}\right) d x .
$$

The "mean free path" of kinecic theory, with particles 1 and 2 both in Maxwel1 distributions involves the mere tractable integral

$$
\bar{v}_{1}^{\prime}=\iint\left|v_{1}-v_{2}\right| F_{1}\left(V_{1}\right) F_{2}\left(V_{2}\right) d V_{1} d V_{2}=(8 K T / \pi \mu)^{\frac{1}{2}}
$$

where

$$
\mu=m_{1} m_{2} /\left(m_{1}+m_{2}\right)
$$

Note 2. The cross section (9) for the case of a photon beam reduces to

where

$$
s\left(k_{1}\right)=\left(\frac{1}{2}\right) \int_{0}^{\infty} \int_{-1}^{1} s^{r}\left(k_{1}^{\prime}\right)\left\{1-\left(c p_{2} / E_{2}\right) a\right\} f\left(k_{2}\right) d k_{2} d a
$$

$$
k_{1}^{\prime}=k_{1}\left(E_{2}-c p_{2}^{a}\right) / e_{2} \text {. Here in the (physically uninteresting) case }
$$
$s^{r}\left(k_{1}^{\prime}\right)=s^{r}$ constant, one sees that $s\left(k_{1}\right) \equiv s^{r}$ regardless of $f\left(k_{2}\right)$. Hence, for a photon crossing Times Square.... 
Note 3. Unfortunately no reference for (9) has been found, and some manifest subtleties may vitiate the result, which is offered tentatively. It should be emphasized that in any case the "effective cross section" considered here is not Lorentz invariant but is only a means to a free path, upon which depends the Monte Carlo position of first collision. In the following section we revert to standard practice, regarding (non-differential) cross sections as intrinsic properties of the target particle, as measured in its rest frame.

37. Transformation of differential cross sections. Consider the passage of a projectile 1 of $k_{\text {.e. }} k_{1}>0$, through a medium at rest in $\Sigma$, of total density $n$ particles $/ \mathrm{cm}^{3}$, of which the fraction $f_{h}$ are of type $h$. Then the Monte Car1o determination of distance to collision depends on the free path $L=1 / n s$, where $s=\Sigma f_{h} s_{h}$ is the "effective cross section," and the $s_{h}=s_{h}\left(k_{i}\right)$ are the individual rest cross sections, as discussed in 536 .

In the event of a collision, the type $h$ of particle hit depends on the probability $n_{h} s_{h} / n s=f_{h} s_{h} / s$. The collision of 1 with a definite type $h$ of particle may result in various kinds of reactions $r$, each with its own cross section $\mathrm{s}_{\mathrm{hr}}$, the probability of reaction $\mathrm{r}$ being $\mathrm{s}_{\mathrm{hr}} / \mathrm{s}_{\mathrm{h}}$.

Now, supposing that the type $h$ and reaction $r$ have been decided upon, we focus attention on the particular reaction involved,

$$
A\left(e_{1}, e_{h}\right) \rightarrow S\left(e_{i}\right)
$$

with cross section $s_{h r}$, and threshold

$$
k_{T}=\frac{e_{a}+e_{s}}{2 e_{h}}(-Q), Q=e_{a}-e_{s}
$$


I. We first suppose that the reaction ( $r$ ) results in a system $S\left(e_{i}\right)$ of $I \geq 3$ particles, of which a definite number $\mu_{j} \geq 1$ are of the type $j$. We may then speak of the probability $f(E, \Psi) d E d \Psi$ of emission of a $j$-particle on the range $(E, E+d E),(\Psi, \Psi+d \Psi)$ in $\Sigma$, the variables $E, \Psi$ being independent since $I \geq 3$. The corresponding "production" differential cross section is then

$$
\sigma(E, \Psi)=\mu_{j} s_{h r} f(E, \Psi)
$$

with

$$
\iint \sigma(E, \Psi) \mathrm{dEd} \Psi=\mu_{j} s_{h r}
$$

If $f^{\prime}\left(E^{\prime}, \Psi^{\prime}\right) d E^{\prime} d \Psi^{\prime}$ denctes the corresponding probability of $j$-particle emission in the reaction

$$
A^{\prime}\left(e_{1}, e_{h}\right)+S^{\prime}\left(e_{i}\right)
$$

as it appears in a frame $\Sigma^{\prime}$ moving at constant velocity $v_{0}=v_{0} \Psi_{0}$ relative to $\Sigma$, then the equation

$$
f^{\prime}\left(E^{\prime}, \Psi^{\prime}\right) d E^{\prime} d \Psi^{\prime}=f(E, \Psi) d E d \Psi
$$

is dictated by the invariance of $\mathrm{j}$-particle counts. Regarding $\mathrm{s}_{\mathrm{hr}}$ as invariant, the same relation is seen to govern the differential cross sections $\sigma^{\prime}$ and $\sigma$.

For the standard axes of $\$ 5$, and polar coordinates $(\psi, \phi),\left(\psi^{\prime}, \phi^{\prime}\right)$ for location of $\Psi, \Psi^{\prime}$ about $X=\Psi_{0}=X^{\prime}$, we may write (1) in the form 
$f^{\prime}\left(E^{\prime}, \psi^{\prime}, \phi^{\prime}\right) d E^{\prime} \sin \psi^{\prime} d \psi^{\prime} d \phi^{\prime}=f(E, \psi, \phi) d E \sin \psi d \psi d \phi$

Setting $a^{\prime}=\cos \psi^{\prime}, a=\cos \psi$, and noting that $\phi^{\prime}=\phi$ for standard axes, this becomes

$$
f^{\prime}\left(E^{\prime}, a^{\prime}, \phi\right) d E^{\prime} d a^{\prime}=f(E, a, \phi) d E d a
$$

It follows that

$$
f^{\prime}\left(E^{\prime}, a^{\prime}, \phi\right) d E^{\prime} d a^{\prime}=f(E, a, \phi)\left|\partial(E, a) / \partial\left(E^{\prime}, a^{\prime}\right)\right| d E^{\prime} d a^{\prime}
$$

where the barred factor denotes the absolute value of the Jacobian

$$
J=\operatorname{det}\left[\begin{array}{ll}
\partial E / \partial E^{\prime} & \partial a / \partial E^{\prime} \\
\partial E / \partial a^{\prime} & \partial a / \partial a^{\prime}
\end{array}\right]
$$

of the transformation $E=E\left(E^{\prime}, a^{\prime}\right), a=a\left(E^{\prime}, a^{\prime}\right)$ from $\Sigma^{\prime}$ to $\Sigma$. The latter is concealed implicitly in the (cPE) transformation

$$
\begin{aligned}
(c p) a & \left.=\gamma_{0}\left\{\left(c p^{\prime}\right) a^{\prime}+\beta_{0} E^{\prime}\right)\right\} \\
E & =\gamma_{0}\left\{\beta_{0}\left(c p^{\prime}\right) a^{\prime}+E^{\prime}\right\}
\end{aligned}
$$

where $(c p)^{2}=E^{2}-e^{2},\left(c p^{\prime}\right)^{2}=E^{\prime 2}-e^{2}$.

$$
\text { From }(c p)^{2}=E^{2}-e^{2} \text { follows } d(c p) / d E=E /(c p)
$$

and hence by the product rule,

$$
\begin{aligned}
& \frac{\partial}{\partial E^{\prime}}(c p) a=E /(c p) \cdot\left(\partial E / \partial E^{\prime}\right) \cdot a+(c p)\left(\partial a / \partial E^{\prime}\right) \\
& \frac{\partial}{\partial a},(c p) a=E /(c p) \cdot\left(\partial E / \partial a^{\prime}\right) \cdot a+(c p)\left(\partial a / \partial a^{\prime}\right)
\end{aligned}
$$


Thus multiplying the second column in (4) by (CP), and then adding to the result the multiple $E /(c p) \cdot a$ of the first, we see that

$$
(c p) J=\operatorname{det}\left[\begin{array}{ll}
\partial E^{\prime} / \partial E^{\prime} & \partial(c p) a / \partial E^{\prime} \\
\partial E^{\prime} \partial a^{\prime} & \partial(c p) a / \partial a^{\prime}
\end{array}\right]
$$

These partials are readily found from (5) to be, respectively,

$$
\begin{array}{ll}
\gamma_{0}\left\{\beta_{0}\left(E^{\prime} / C p^{\prime}\right) a^{\prime}+1\right\} & \gamma_{0}\left\{\left(E^{\prime} / c p^{\prime}\right) a^{\prime}+\beta_{0}\right\} \\
\gamma_{0}\left\{\beta_{0}\left(c p^{\prime}\right)\right\} & \gamma_{0}\left\{c p^{\prime}\right\}
\end{array}
$$

so that $(c p) J=c p^{\prime}$. Thus (3) reduces to the symmetric relation

$$
f^{\prime}\left(E^{\prime}, a^{\prime} \phi\right) d E^{\prime} d a^{\prime} / c p^{\prime}=f(E, a, \phi) d E d a / c p
$$

Analogous formulas obtain for other variables. Thus one may show in similar fashion

$$
f^{\prime}\left(c p^{\prime}, a^{\prime}, \phi\right) E^{\prime} d\left(c p^{\prime}\right) d a^{\prime} /\left(c p^{\prime}\right)^{2} \equiv f(c p, a, \phi) E d(c p) d a /(c p)^{2}
$$

II. The condition $I \geq 3$ imposed on the system $S\left(e_{i}\right)$ was required for the independence of the variables $E^{\prime}, a^{\prime}$. For a two particle system $S\left(e_{i}\right)$, $E^{\prime}$ is uniquely determined by the critical energy $e_{0}$ of the class of $A$, and the two proper energies $e_{i}(520)$, and one speaks of a probability of emission

$$
f^{\prime}\left(a^{\prime}, \phi\right) d a^{\prime} d \phi=f(a, \phi) d a d \phi
$$


of either one of the $e_{i}$, so that here

$$
f^{\prime}\left(a^{\prime}, \phi\right) d a^{\prime}=f(a, \phi)\left(\mathrm{da} / \mathrm{da} a^{\prime}\right) \mathrm{da} a^{\prime}
$$

We have derived the required formulas in $\$ 9$, namely

$$
\begin{aligned}
& a=\gamma_{0}\left(a^{\prime}+\rho^{\prime}\right) / \gamma_{0} D^{\prime}, \quad \rho^{\prime}=\beta_{0} / B^{\prime}=B_{0} E^{\prime} / c p^{\prime} \\
& \mathrm{da} / \mathrm{da} a^{\prime}=\left(1+\rho^{\prime} \mathrm{a}^{\prime}\right) / \gamma_{0}^{2} D^{, 3}
\end{aligned}
$$

where

$$
D^{\prime}=\left\{\left(a^{\prime}+\rho^{\prime}\right)^{2}+\gamma_{0}^{-2}\left(1-a^{\prime 2}\right)\right\}^{\frac{1}{2}}
$$

The parameters here refer to the particle $i$ being considered. In case it is immaterial, we have the simple version

$$
\begin{aligned}
& a=\gamma_{0}\left(a^{\prime}+\beta_{0}\right) / \gamma_{0} d^{\prime} \\
& d a / d a^{\prime}=1 / \gamma_{0}^{2} d^{\prime 2} \\
& d^{\prime}=1+\beta_{0} a^{\prime}
\end{aligned}
$$

Note 1. For a more complete discussion, including singularities, see K. G. Dedrick, Rev. Mod. Phys. 34, 1962, 429-442. 


\section{APPENDIX I}

\section{COORDINATES AND ROTATIONS}

By a set of axes in a Euclidean 3-space of vectors $R$, we mean here any right-handed ordered set $A=[X, Y, Z]$ of mutually perpendicular unit vectors $X, Y, Z$. The relation

$$
R=X X+Y y+Z z=A\left[\begin{array}{l}
x \\
y \\
z
\end{array}\right]
$$

then determines $x, y, z$ as the A-coordinates of $R$, and we write

$$
R=(x, y, z)_{A}
$$

A rotation $\delta$ (intuitively a "rigid motion" about the origin) is completely defined by its action on any set of axes $A$, as indicated by an equation of form

$$
\delta A=[\delta X, \delta Y, \delta Z]=[X, Y, Z] D=A \cdot D
$$

where $D=\left[d_{i j}\right]$ is a $3 \times 3$ matrix with $D^{-1}=D^{\tau} \equiv\left[d_{j i}\right]$, and $\operatorname{det} D=+1$. The vectors $[\delta X, \delta Y, \delta Z]$ then form a set of axes a1so, with A-coordinates given by the columns of $D$.

Theorem 1. Let $A=[X, Y, Z]$ be a set of axes, $\delta$ a rotation defined by $\delta A=A D$, and $R=(x, y, z)_{A}$ a point with A-coordinates $(x, y, z)$. Then

(a) $\delta R$ has A-coordinates $\left(x^{\prime}, y^{\prime}, z^{\prime}\right)$ given by

$$
\left[\begin{array}{l}
x \\
y \\
z
\end{array}\right]=D\left[\begin{array}{l}
x \\
y \\
z
\end{array}\right]
$$


(b) $R$ has $\delta A$-coordinates $(\bar{x}, \bar{y}, \bar{z})$ given by

$$
\left[\begin{array}{l}
\bar{x} \\
\bar{y} \\
z
\end{array}\right]=D^{\tau}\left[\begin{array}{l}
x \\
y \\
z
\end{array}\right]
$$

Proof. From $R=A\left[\begin{array}{l}x \\ y \\ z\end{array}\right]$ and $\delta A=A D$ follows
(a) $\delta R=\delta \cdot A\left|\begin{array}{l}x \\ y \\ z\end{array}\right|=A \cdot D\left|\begin{array}{l}x \\ y \\ z\end{array}\right|=A\left|\begin{array}{l}x^{\prime} \\ y^{\prime} \\ z^{\prime}\end{array}\right|$
(b) $R=A\left|\begin{array}{l}x \\ y \\ z\end{array}\right|=\delta A \cdot D^{\tau}\left|\begin{array}{l}x \\ y \\ z\end{array}\right|=\delta A\left|\begin{array}{l}\bar{x} \\ \bar{y} \\ z\end{array}\right|$

See Fig. 1.

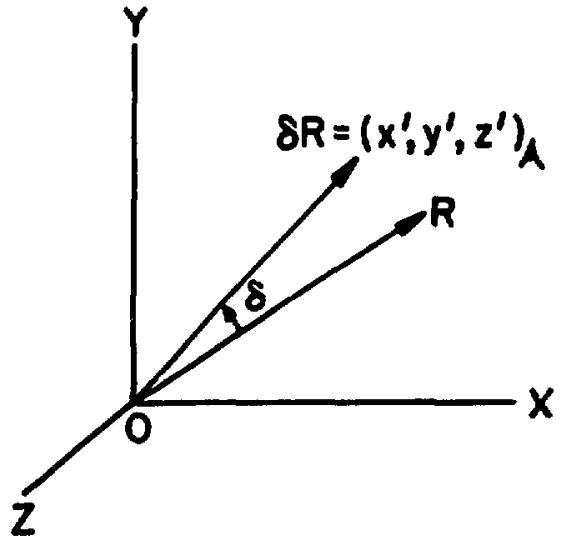

(a)

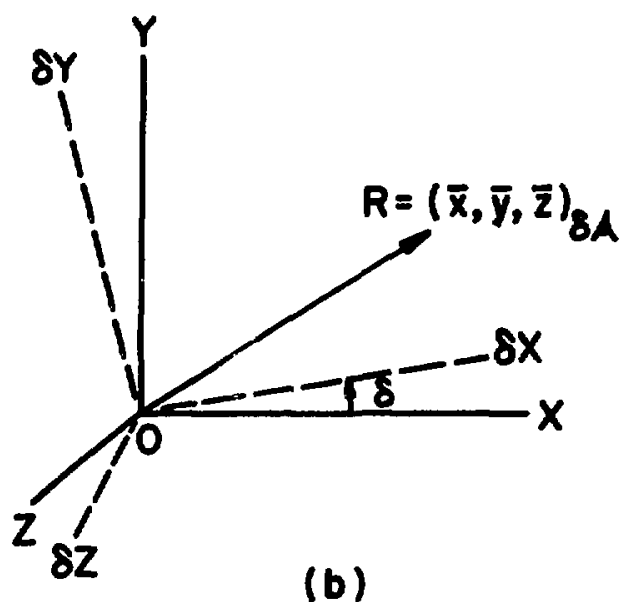

(b)

Fig. 1 .

$\delta R$ on $A$ and $R$ on $\delta A$ 
Every rotation $\delta$ may be achieved by a right-handed rotation through an angle $\theta\left(0^{\circ} \leq \theta \leq 180^{\circ}\right)$ about some fixed unit vector $\hat{\Psi}$, defined as in

Theorem 2. If a unit vector $\hat{\psi}$ has $A$-coordinates $\left(b_{x}, b_{y}, b_{z}\right)$, and $\theta$ is given on $\left[0^{\circ}, 180^{\circ}\right]$, with

$$
\mathrm{C} \equiv \cos \theta, \quad \mathrm{C}^{\prime} \equiv 1-\cos \theta, \quad \mathrm{S} \equiv \sin \theta(\geq 0)
$$

then the right-handed rotation $\delta$ about $\hat{\Psi}$ through $\theta$ is defined by $\delta A=A D$, where

$$
D=\left[\begin{array}{lll}
C+b_{x}^{2} c^{\prime} & -b_{z} s+b_{y} b_{x} c^{\prime} & b_{y} s+b_{z} b_{x} c^{\prime} \\
b_{z} s+b_{x} b_{y} c^{\prime} & c+b_{y}^{2} c^{\prime} & -b_{x} s+b_{z} b_{y} c^{\prime} \\
-b_{y} s+b_{x} b_{z} c^{\prime} & b_{x} s+b_{y} b_{z} c^{\prime} & c+b_{z} c^{\prime}
\end{array}\right]
$$

Proof. By symmetry, it suffices to verify that the first column of D gives the correct $A$-coordinates of $\delta x$. Let

$$
\begin{aligned}
& x_{\|}=(x \cdot \hat{\Psi}) \hat{\Psi}=\left(b_{x}^{2}, b_{x} b_{y}, b_{x} b_{z}\right)_{A} \\
& x_{1}=x-x_{\|}=\left(1-b_{x}^{2},-b_{x} b_{y},-b_{x} b_{z}\right)_{A}
\end{aligned}
$$

where $\left|x_{1}\right|=\left(1-b_{x}^{2}\right)^{\frac{1}{2}}$

Then the required $\delta \mathrm{X}$ is

$$
\delta \mathrm{X}=\delta\left(\mathrm{X}_{\mathrm{y}}+\mathrm{X}_{1}\right)=\mathrm{X}_{\mathrm{y}}+\delta \mathrm{X}_{1} \text {, where }\left|\delta \mathrm{X}_{1}\right|=\left|\mathrm{x}_{1}\right|
$$




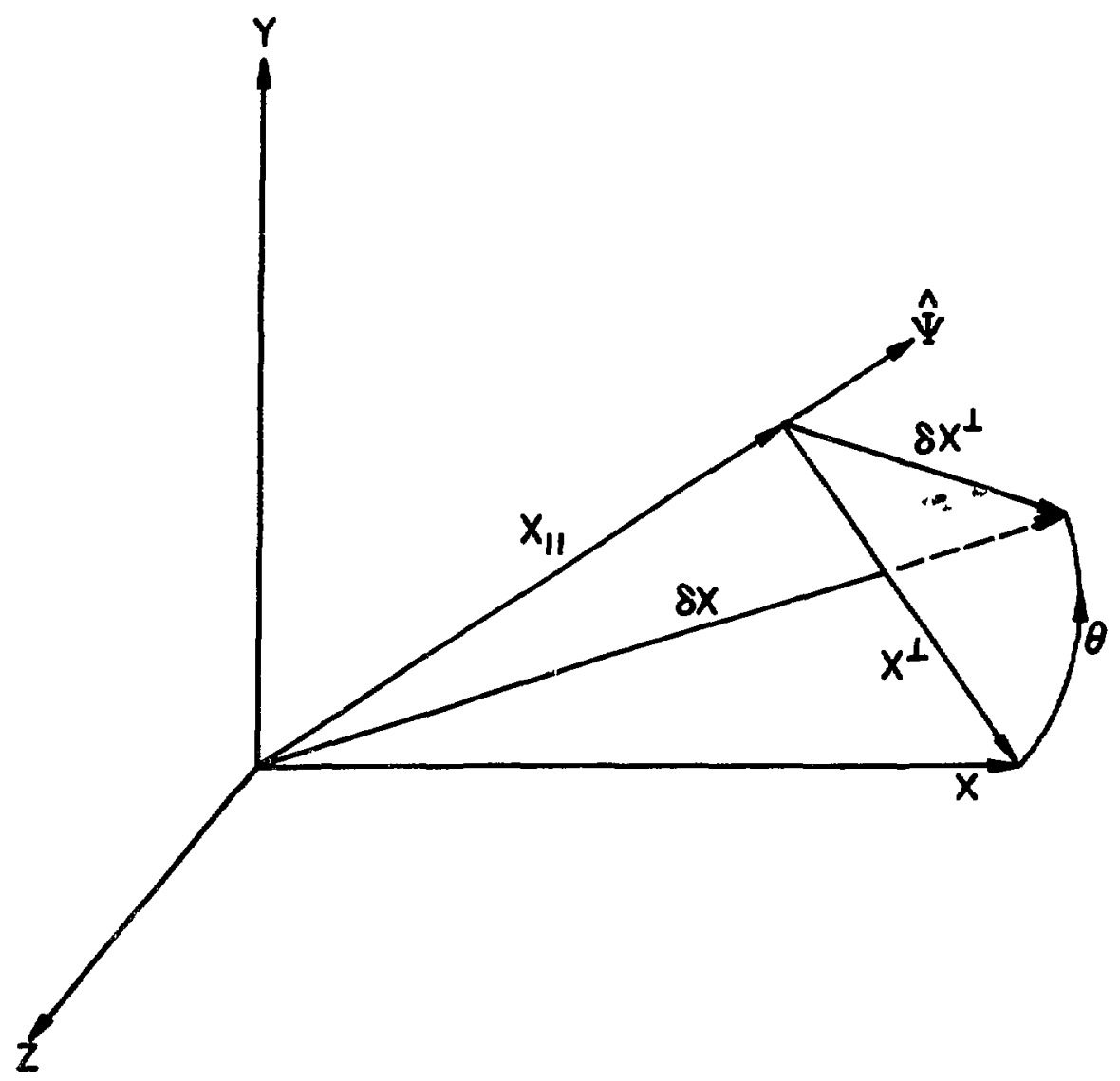

Fig. 2.

- Rotation about an axis 
It is now geometrically clear from Fig. 2 that $\delta x_{1}$ must satisfy the following (dependent) conditions.

$$
\begin{aligned}
& \hat{\psi} \cdot \delta x_{\perp}=0 \\
& x_{\perp} \cdot \delta x_{\perp}=\left|x_{\perp}\right|^{2} \mathrm{C} \\
& x_{\perp} \times \delta x_{\perp}=\left|x_{\perp}\right|^{2} s \hat{\psi}
\end{aligned}
$$

provided $x_{\perp} \neq 0, S \neq 0$. (In the trivial cases $x_{\perp}=0, \hat{\Psi}=x$, and $S=0$, $\theta=0^{\circ}$ or $180^{\circ}$, the matrix $D$ is easily seen to give the correct result. In case $\theta=180^{\circ}$, one should note that $\delta X_{1}=-X_{1}$, and hence $\delta X=X_{\|}-X_{1} \cdot$ ) Letting $\delta x_{1}=\left(p_{x}, p_{y}, p_{z}\right)_{A}$, these conditions imply

$$
\begin{aligned}
& b_{x} p_{x}+b_{y} p_{y}+b_{z} p_{z}=0 \\
& \left(1-b_{x}^{2}\right) p_{x}-b_{x} b_{y} p_{y}-b_{x} b_{z} p_{z}=\left(1-b_{x}^{2}\right) c \\
& b_{x} b_{z} p_{y}-b_{x} b_{y} p_{z}=\left(1-b_{x}^{2}\right) S b_{x}
\end{aligned}
$$

From (13), (14) we have at once

$$
p_{x}=\left(1-b_{x}^{2}\right) c
$$

Since $\left(1-b_{x}^{2}\right)^{\frac{1}{2}}=\left|x_{1}\right| \neq 0$ by agreement, we then obtain from (13), the values 


$$
p_{y}=b_{z} s-b_{x} b_{y} c, \quad p_{z}=-b_{y} s-b_{x} b_{z} c
$$

provided $b_{x} \neq 0$. If $b_{x}=0$, Eqs. (17) are seen to hold directly from the full product (12).

From (9a), (7), (16), (17), we find that

$$
\begin{aligned}
\delta x & =x_{\|}+\delta x_{I}=\left(b_{x}^{2}, b_{x} b_{y}, t_{x} b_{z}\right)_{A}+\left(p_{x}, p_{y}, p_{z}\right)_{A} \\
& =\left(C+b_{x}^{2} C^{\prime}, b_{z} S+b_{x} b_{y} C^{\prime},-b_{y} S+b_{x} b_{z} C^{\prime}\right)_{A},
\end{aligned}
$$

which verifies the first column of $D$.

Corollary 1. Let $\left(a_{x}, a_{y}, a_{z}\right)$ be the A-coordinates of a given unit vector $\Psi$. Then a rotation $\delta$ which takes $X$ into $\Psi$ is defined by $\delta A=A D$, wher 6
(a) $\quad D=\left[\begin{array}{lll}a_{x} & -a_{y} & -a_{z} \\ a_{y} & 1-\left(a_{y}^{2} / \Delta\right) & -a_{y} a_{z} / \Delta \\ a_{z} & -a y_{z} / \Delta & 1-\left(a_{z}^{2} / \Delta\right)\end{array}\right], \Delta \equiv 1+a_{x} \neq 0$
(b) $D=\left[\begin{array}{rrr}-1 & 0 & 0 \\ 0 & -1 & 0 \\ 0 & 0 & 1\end{array}\right], \Delta=1+a_{x}=0$

Proof. If $a_{x}=1$, then $\Psi=X$, and $D=I$ in (a). If $a_{x}=-1$, then $\Psi=-X$, and the $D$ in (b) defines a rotation about $Z$ which takes $X$ into $\Psi$. Otherwise $\Psi \neq \pm X, X$ and $\Psi$ determine a plane, and a right-handed rotation $\delta$, 
through an angle $\theta$ on $\left(0^{\circ}, 180^{\circ}\right)$ with $\mathrm{C}=\cos \theta=\mathrm{X} \cdot \Psi=\mathrm{a}_{\mathrm{x}}$, about its normal $\hat{\Psi}=(X \times \Psi) / S=\left(0,-a_{z}, a_{y}\right) / S, S=\sin \theta=\left(1-a_{x}^{2}\right)^{\frac{1}{2}}>0$, will have $\delta X=\Psi$ as required (Fig. 3). The matrix $D$ in (a) results from that of Theorem 2 upon making these substitutions.

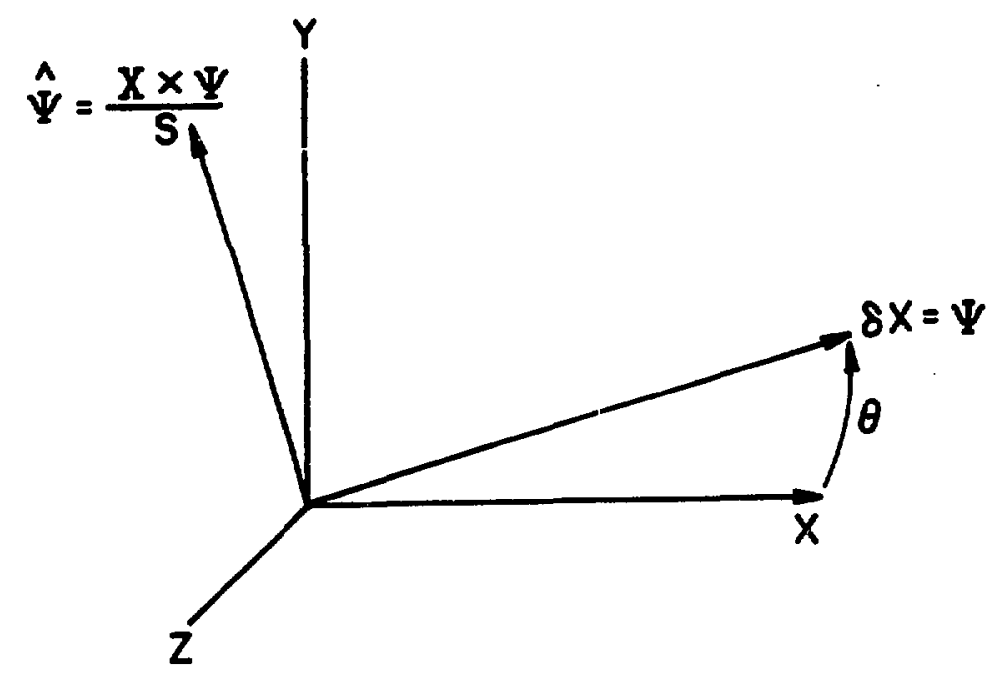

Fig. 3. A rotation taking $X$ into $\Psi$

Corollary 2. If $\Psi=\left(a_{x}, a_{y}, a_{z}\right)_{A}$ is a given unit vector then the rotation $\delta_{1}$ defined by $\delta_{1} A=A D_{1}$ where

$$
\begin{aligned}
& D_{1}=\left[\begin{array}{ccc}
a_{x} & a_{y} & a_{z} \\
a_{y} & \left(a_{y}^{2} / \Delta\right)-1 & a_{y} a_{z} / \Delta \\
a_{z} & a_{y z} a_{z} / \Delta & \left(a_{z}^{2} / \Delta\right)-1
\end{array}\right], \Delta=1+a_{x} \neq 0 \\
& D_{1}=\left[\begin{array}{rlr}
-1 & 0 & 0 \\
0 & 1 & 0 \\
0 & 0 & -1
\end{array}\right], \Delta=1+a_{x}=0
\end{aligned}
$$

also takes $X$ into $\Psi$, and moreover $D^{\tau}=D$. 
Proof. If $\delta^{*}$ is the rotation about $X$ through $180^{\circ}$, defined by $\delta * A=A D^{*}$, where

$$
D^{*}=\left[\begin{array}{rrr}
1 & 0 & 0 \\
0 & -1 & 0 \\
0 & 0 & -1
\end{array}\right]
$$

and $\delta$ is the rotation of Corollary 1 , then

$$
\delta \delta^{*} A=\delta A D^{*}=A D D^{*}=A D_{1}=\delta_{1} A \text {. Hence } \delta_{1} \text { is the rotation } \delta^{*} \text { followed }
$$
by $\delta$.

It is sometimes convenient to make a rotation which not only takes $X$ into a specified vector $\Psi$, but also has the property that a second given vector $\Psi_{1}$ lies in the new $\delta X, \delta Y$ plane. For this we have

Theorem 3. Let $\Psi=\left(a_{x}, a_{y}, a_{z}\right)_{A}, \Psi_{1}=\left(a_{1 x}, a_{1 y}, a_{1 z}\right)_{A}$ be given unit vectors, and define

$$
\mathrm{C}=\cos \psi_{1}=\psi \cdot \psi_{1}, 0^{\circ} \leq \psi_{1} \leq 180^{\circ} \text {, and } \mathrm{S}=\sin \psi_{1}=\left(1-\mathrm{C}^{2}\right)^{\frac{1}{2}} \geq 0 \text {. }
$$

Then we obtain a rotation $\delta_{2}$, defined by $\delta_{2} A=A D_{2}$, such that (a) $\delta_{2} X=\Psi$, and (b) $\Psi_{1}$ lies in the "upper half" of the $\delta_{2} X, \delta_{2} Y$ plane, with $\delta_{2} A$-coordinates $(C, S, 0)$, provided we take $D_{2}$ as the $D$ of Corollary 1 in the trivial case $\Psi_{1}= \pm \Psi$, and otherwise set

$$
D_{2}=\left[\begin{array}{lll}
a_{x} & b_{x} & c_{x} \\
a_{y} & b_{y} & c_{y} \\
a_{z} & b_{z} & c_{z}
\end{array}\right]
$$


where

$$
\begin{array}{ll}
b_{x}=\left(a_{1 x}-a_{x} c\right) / S & c_{x}=\left(a_{y} a_{1 z}-a_{z} a_{1 y}\right) / S \\
b_{y}=\left(a_{1 y}-a_{y} c\right) / S & c_{y}=\left(a_{z} a_{1 x}-a_{x} a_{1 z}\right) / S \\
b_{z}=\left(a_{1 z}-a_{z} c\right) / S & c_{z}=\left(a_{x} a_{1 y}-a_{y} a_{1 x}\right) / S
\end{array}
$$

Proof. The matrix $D_{2}$ is completely determined in the non-trivial case by the conditions

$$
\delta_{2} X=\Psi, \quad \delta_{2} Z=\Psi \times \Psi_{1} / S, \quad \delta_{2} Y=\delta_{2} Z \times \delta_{2} X \text { (Fig. 4) }
$$

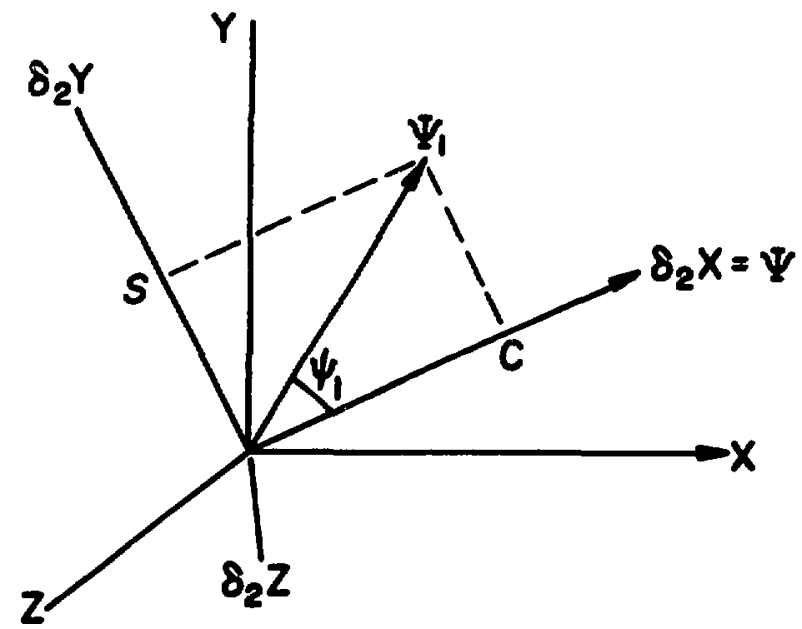

Fig. 4. Rotation into a plane

The first condition gives the first column of $D_{2}$, the second gives the third column, and the third condition then gives the second column, using the relations $a_{y}^{2}+a_{y}^{2}+a_{z}^{2}=1$, and $a_{x} a_{1 x}+a_{y} a_{1 y}+a_{z} a_{1 z}=c$.

Corollary 3. Let $\Psi=\left(a_{x}, a_{y}, a_{z}\right)_{A}, \Psi_{1}=\left(a_{1 x}, a_{1 y}, a_{1 z}\right)_{A}$ be perpendicular unit vectors. Then the rotation $\delta_{2}$, defined by $\delta_{2} A=A D_{2}$, where 


$$
D_{2}=\left[\begin{array}{lll}
a_{x} & a_{1 x} & a_{y} a_{1 z}-a_{z} a_{1 y} \\
a_{y} & a_{1 y} & a_{z} a_{1 x}-a_{x} a_{1 z} \\
a_{z} & a_{1 z} & a_{x} a_{1 y}-a_{y} a_{1 x}
\end{array}\right]
$$

is a rotation such that $\delta_{2} X=\Psi$ and $\delta_{2} Y=\Psi_{1}$.

Proof. Set $C=0, S=1$ in Theorem 3 . 


\section{APPENDIX II}

\section{A STANDARD DEVICE FOR SCATTERING}

Suppose a basic direction $\Psi=\left(a_{x}, a_{y}, a_{2}\right)_{A}$ is given relative to axes $A$ in a frame $\Sigma$, and a second direction $\Psi_{i}$, in a "non-polarized" distribution about $\Psi$, is to be chosen by sampling. This means, in effect, that the latitude angle $\theta, 0^{\circ} \leq \theta \leq 180^{\circ}$, which $\Psi_{i}$ makes with $\Psi$ may be drawn from a given distribution, and that a second longitudinal angle $\phi$, uniformly distributed on $\left[0^{\circ}, 360^{\circ}\right)$, and measured from any plane through $\Psi$, may be used to locate $\Psi_{i}$ on the cone of angular opening $\theta$ with axis $\Psi$. (Fig. 1.) Since the auxiliary direction

$$
\Omega=(\cos \theta, \sin \theta \cos \phi, \sin \theta \sin \phi)_{A}
$$

is distributed about $X$ as $\Psi_{i}$ should be about $\Psi$, it is clear that $\Psi_{i}$ may be chosen as the vector $\Psi_{i}=\delta \Omega$ where $\delta$ is any rotation which takes $\mathrm{X}$ into $\Psi$. The explicit rotation $\delta$ of AI, Cor. 1, based on the A-coordinates of $\Psi$, and having the matrix given there as $D$, will serve this purpose, and the A-coordinates of $\Psi_{i}$ are given by

$$
\left(\Psi_{i}\right)_{A}=D(\Omega)_{A}
$$

as indicated in Theorem 1 (a) of the same Appendix.

The given basic direction $\Psi$ frequently coincides with $X$, and may always be so chosen in case of isotropic scattering. In the latter case, $\cos \theta$ is equi-d1stributed on $[-1,1]$, and one simply sets

$$
\left(\Psi_{i}\right)_{A}=(\Omega)_{A}
$$




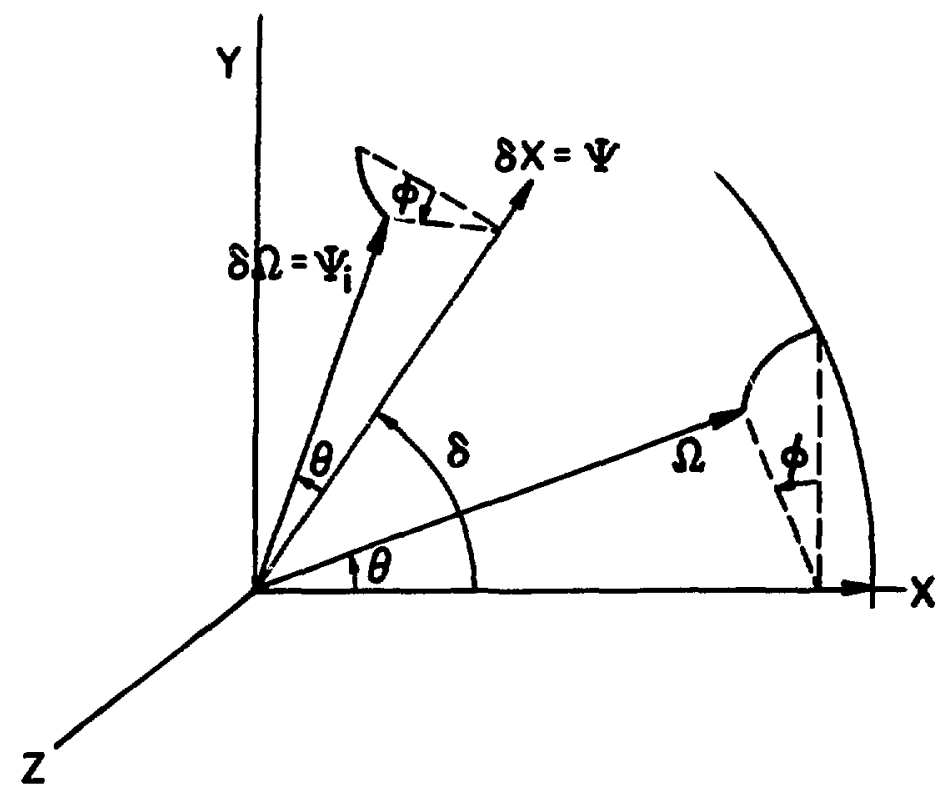

Fig. 1.

A Standard Device 


\section{APPENDIX III}

\section{A GENERAL LORENTZ TRANSFORMATION}

Let $\Sigma^{\prime}=R^{n+1}$ be an "event space" of vectors $\xi^{\prime}=\left|X_{\tau}^{X^{\prime}}\right|$, where $X^{\prime}$ is a "position" in $R^{n}$ and $\tau^{\prime} \in R^{1}$. A Lorentz transformation (L.T.) means here any non-singular 1 inear transformation $(n+1$ order matrix)

$$
T=\left[\begin{array}{ll}
A & B \\
C^{\tau} & d
\end{array}\right]
$$

of $\Sigma$ with the property

(L) $\left\|X^{\prime}\right\|=\left|\tau^{\prime}\right|$ for $\xi^{\prime}=\mid$\begin{tabular}{|l}
$X^{\prime}$ \\
$\tau^{\prime}$
\end{tabular} implies $\left\|x_{\|}\right\|=|\tau|$ for the corresponding

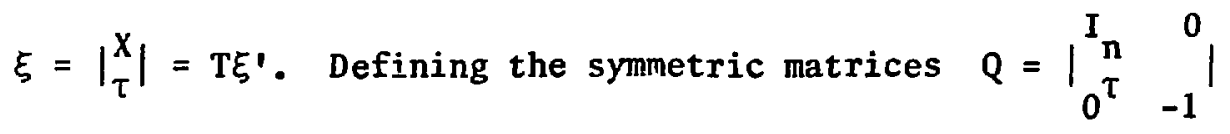
and $P=T^{\tau} Q T=\left[p_{i j}\right]$, we have the relations

$$
\begin{aligned}
& \left\|X^{\prime}\right\|^{2}-\tau \prime^{2} \equiv \sum_{1}^{n} x_{i}^{\prime 2}-\tau^{\prime 2} \equiv \xi^{\prime}{ }^{\tau} Q \xi^{\prime} \\
& \|x\|^{2}-\tau^{2} \equiv \Sigma_{1}^{n} x_{i}^{2}-\tau^{2} \equiv \xi^{\tau} Q \xi=\left(T \xi^{\prime}\right)^{\tau} Q\left(T \xi^{\prime}\right)=\xi^{\prime} \tau_{P} \xi^{\prime}
\end{aligned}
$$

Hence (L) may be expressed in the form

(L') $\xi^{\prime}{ }^{\top} \mathrm{Q} \xi^{\prime}=0$ implies $\xi^{\prime}{ }^{\top} \mathrm{P} \xi^{\prime}=0$ where $\mathrm{P}=\mathrm{T}^{\tau} \mathrm{QT}$.

Theorem 1. If $\mathrm{T}$ is a L.T., then

$$
\mathrm{T}^{\tau} \mathrm{QT}=\mathrm{qQ}
$$

and

$$
\Sigma_{1}^{n} x_{i}^{2}-\tau^{2} \equiv q\left(\Sigma_{1}^{n} x_{i}^{\prime 2}-\tau^{2}\right)
$$


-250-

where

$$
q=d^{2}-\|B\|^{2} \neq 0
$$

Conversely if $T$ is a matrix such that $T^{\tau} Q T=q Q$ with $q \neq 0$, then $T$ is a LoT.

Proof. Let $\delta_{i}$ be a position vector with $i$-th component 1 , all others

0 . Since $X^{\prime}= \pm \delta_{i}, \tau^{\prime}=1$, and $X^{\prime}=3 \delta_{i}+4 \delta_{j}, \tau^{\prime}=5$ all satisfy $\xi^{\prime}{ }^{\tau} Q \xi^{\prime} \equiv \Sigma_{1}^{n} x_{i}^{\prime 2}-\tau^{\prime 2}=0$, it follows from (L') that $\xi^{\prime}{ }^{\prime} P \xi^{\prime}=0$, and computation shows that

$$
\begin{aligned}
& p_{i, n+1}=0, p_{i i}=-p_{n+1, n+1}, i=1, \ldots, n \text { and } p_{i j}=0, i \neq j, \\
& i, j=1, \ldots, n \text {. }
\end{aligned}
$$

Hence $P=T^{\tau} Q T=-p_{n+1, n+1} Q \equiv q Q$, where $q \neq 0$ since $\operatorname{det} T \neq 0$. From $P=q Q$ and (1), (2), identity (4) follows, and the value of $q$ in (5) is obtained from (4) by substituting $X^{\prime}=0, \tau^{\prime}=1$ and $X=A 0+B \cdot 1=B, \tau=c^{\tau} 0+d \cdot 1=d$. Conversely, $T^{\tau} Q T=q Q, q \neq 0$ implies (4) and $(L)$ is a consequence.

Corollary 1. The set of all L.T.'s is a group.

Proof. This follows formally from the $N$. \& $S$. condition $T^{T} Q T=q Q, q \neq 0$.

Corollary 2. The matrix $T$ is a L.T. inf (6) $A^{\tau} A-C C^{\tau}=q I_{n}$

(7) $A^{\tau} B=d C$ where (8) $q=d^{2}-\|B\|^{2} \neq 0$.

Proof. The result follows from Theorem 1 by block multiplication.

Corollary 3. T is a L.T. Af it is of form

$$
\mathrm{T}=\mathrm{dT} \mathrm{T}_{1} \equiv \mathrm{d}\left[\begin{array}{cc}
\mathrm{A}_{1} & \mathrm{~B}_{1} \\
\mathrm{C}_{1}^{\tau} & 1
\end{array}\right], \mathrm{d} \neq 0
$$

where (9) $A_{1}^{\tau} A_{1}-C_{1} C_{1}^{\tau}=q_{1} I_{n} \quad$ (10) $A_{1}^{\tau} B_{1}=C_{1}$ and (11) $q_{1}=1-\left\|B_{1}\right\|^{2} \neq 0$. 
Proof. Suppose $d=0$ in the L.T. T. Let $X '$ be a non-zero vector (existence obvious) such that $C^{\tau} X^{\prime}=0$, and define $\tau^{\prime}=\left\|X^{\prime}\right\|$. Then $\|x \cdot\|=|\tau \cdot|$, so we must a1so have

$$
\left\|A X^{\prime}+B \tau^{\prime}\right\|=\|X\|=|\tau|=\left|C^{\tau} X^{\prime}+d \tau^{\prime}\right|=0 \text {. But a non-singular matrix } T
$$

cannot take a non-zero vector $\left.\right|_{\tau} ^{X_{j}^{\prime}} \mid$ into a zero vector $\left|{ }_{\tau}^{X}\right|$. The rest is clear from Corollary 2.

$$
\text { Corollary 4. If } T \text { is a L.T. as in Corollary } 3 \text {, then } B_{1}=0 \text { iff } C_{1}=0 \text {. }
$$

In such a case, $q_{1}=1$, and $T$ is of form

$$
T=d\left[\begin{array}{ll}
R & 0 \\
0 & 1
\end{array}\right]
$$

where $R^{\tau} R=I_{n}$, i.e., $R$ is a "rotation."

Proof. If $B_{1}=0$ then $C_{1}=0$ by (10). If $C_{1}=0$, then $A_{1}^{\tau} A_{1}=q_{1} I n, q_{1} \neq 0$ by (9) and (11); hence $A_{1}$ is non-singular and $B_{1}=0$ by (10). If $B_{1}=0=C_{1}$, then $a_{1}=1$ by (1i) and $A_{1}$ is rotation by (9).

Definition: A rocation here means any matrix such that $R^{\tau} R=I$. A "space rotation" means any matrix of form

$$
S=\left[\begin{array}{ll}
R & 0 \\
0 & 1
\end{array}\right], \quad R^{\tau_{R}}=I_{n}
$$

The set $S^{*}$ of all such $S$ is a group, and for $S \varepsilon S^{*}$, one has $S^{-1}=S^{\tau}$ and $\operatorname{det} S= \pm 1$

Corollary 5. The set of all matrices of form $d S, d \neq 0, S \varepsilon S^{*}$, is a group, consisting of precisely those L.T.'s with $B=C=0$. 
Proof. This is a consequence of Corollary 4 and Corollary 3. Having characterized the set of all L.T.'s in Corollary 3 and the subset of Corollary 5 , we turn to the non-trivial case $B \neq 0, C \neq 0$. Suppose then that $\mathrm{T}$ is a L.T. as in Corollary 3, with

$$
\left\|B_{1}\right\|=b_{0}>0 \quad\left\|c_{1}\right\|=c_{0}>0
$$

and consider the equation

$$
S_{1} T_{1} S^{\prime} \tau=\left[\begin{array}{ll}
R_{1} & 0 \\
0 & 1
\end{array}\right]\left[\begin{array}{cc}
A_{1} & B_{1} \\
C_{1}^{\tau} & 1
\end{array}\right]\left[\begin{array}{ll}
R^{\prime} & 0 \\
0 & 1
\end{array}\right]=\left[\begin{array}{ll}
R_{1} A_{1} R^{\prime} & R_{1} B_{1} \\
C_{1}^{\tau}, \tau & 1
\end{array}\right] \equiv T_{2}
$$

where $S_{1}$, $S^{\prime}$ are space rotations.

Let $R_{1}, R^{\prime}$ be rotations such that

$$
R_{1} B_{1}=b_{0} \delta_{1} \text { and } R^{\prime} C_{1}=c_{0} \delta_{1}
$$

Then $T_{2}=S_{1} T_{1} S^{T}$ is a L.T. of form

$$
T_{2}=\left[\begin{array}{ll}
A_{2} & b_{0} \delta_{1} \\
c_{0} \delta_{1} \tau & 1
\end{array}\right]
$$

which by Corollary 3 satisfies

$$
A_{2}^{\tau} A_{2}-c_{0}^{2} \delta_{1} \delta_{1}^{\tau}=q_{1} I_{n} \quad \text { (13) } \quad A_{2}^{\tau} \delta_{1}=\left(c_{0} / b_{0}\right) \delta_{1}
$$

$$
q_{1}=1-b_{0}^{2} \neq 0
$$

By (13) $A_{2}$ has the form

$$
A_{2}=\left[\begin{array}{ll}
c_{0} / b_{0} & 0^{\tau} \\
C_{2} & D_{2}
\end{array}\right]
$$

where $D_{2}$ is square of order $n-1$. From (12), (14) we conclude that in $A_{2}$, 
(15) $\left(c_{0} / b_{0}\right)^{2}+c_{2}^{\tau} c_{2}-c_{0}^{2}=q_{1}=1-b_{0}^{2} \neq 0$

(16) $\mathrm{D}_{2}^{\tau} \mathrm{C}_{2}=0^{\tau}$

(17) $D_{2}^{\tau} D_{2}=q_{1} I_{n-1}$

Since $q_{1} \neq 0$, we see from the main diagonal of (17) that

$q_{1}=1-b_{0}^{2}>0$. Defining

$$
\gamma_{0}=\left(1-b_{0}^{2}\right)^{-\frac{1}{2}}=q_{1}^{-\frac{1}{2}}
$$

we see from (17) that $\gamma_{0} D_{2}$ is a rotation $R_{n-1}$ of $R^{n-1}$ :

$$
\gamma_{0} D_{2}=R_{n-1}
$$

from (16) that $c_{2}=0$, and from (15) that $c_{0}=b_{0}$. Collecting these results, we see that

$$
\begin{aligned}
& \mathrm{T}=\mathrm{dT} \mathrm{T}_{1} \\
& \mathrm{~T}_{2}=\mathrm{S}_{1} \mathrm{~T}_{1} \mathrm{~S}^{\mathrm{T}}=\left[\begin{array}{ll}
\mathrm{A}_{2} & \mathrm{~b}_{0} \delta_{1} \\
\mathrm{~b}_{0} \delta_{1}{ }^{\tau} & 1
\end{array}\right]
\end{aligned}
$$

where

$$
A_{2}=\left[\begin{array}{ll}
1 & 0^{\tau} \\
0 & \gamma_{0}^{-1} R_{n-1}
\end{array}\right]
$$

Finally, defining the space rotations

$$
S_{2}=\left[\begin{array}{ll}
R_{2} & 0 \\
0 & 1
\end{array}\right] \quad \text { where } R_{2}=\left[\begin{array}{cc}
1 & 0 \\
0 & k_{n-1}^{\tau}
\end{array}\right]
$$

and $S=S_{2} S_{1}$, a straightforward block multiplication shows that 
-254-

$$
\mathrm{ST}_{1} S^{, \tau}=\mathrm{S}_{2}\left(\mathrm{~S}_{1} \mathrm{~T}_{1} S^{\prime}{ }^{\tau}\right)=S_{2} \mathrm{~T}_{2}=\left[\begin{array}{ll}
\mathrm{R}_{2} \mathrm{~A}_{2} & \mathrm{~b}_{0} \delta_{1} \\
\mathrm{~b}_{0} \delta_{1}{ }^{\tau} & 1
\end{array}\right]
$$

$$
\begin{aligned}
& \text { where } R_{2} A_{2}=\left[\begin{array}{ll}
1 & 0^{\tau} \\
0 & \gamma_{0}^{-1} I_{n-1}
\end{array}\right] \text {. Hence } \\
& \qquad S T_{1} S^{\prime}=\gamma_{0}^{-1}\left[\begin{array}{lll}
\gamma_{0} & 0^{\tau} & \gamma_{0} b_{0} \\
0 & I_{n-1} & 0 \\
\gamma_{0} b_{0} & 0^{\tau} & \gamma_{0}
\end{array}\right] \equiv \gamma_{0}^{-1} L_{0}
\end{aligned}
$$

Theorem 2. Every Lorentz transformation

$$
T=\left[\begin{array}{ll}
A & B \\
C^{\tau} & d
\end{array}\right], B \neq 0
$$

can be written in the form

$$
T=\left(d / \gamma_{0}\right) S^{\tau} L_{0} S^{\prime}
$$

where $S, S$ ' are space rotations, and

$$
\begin{aligned}
& L_{0}=\left[\begin{array}{lll}
\gamma_{0} & 0^{\tau} & \gamma_{0} \beta_{0} \\
0 & I_{n-1} & 0 \\
\gamma_{0} \beta_{0} & 0^{\tau} & \gamma_{0}
\end{array}\right] \\
& \beta_{0}=\|B / d\|^{2}<1, \gamma_{0}=\left(1-\beta_{0}^{2}\right)^{-\frac{1}{2}}>1 \text {. Moreover, in Theorem } 1, q=d^{2} / \gamma_{0}^{2} \text {. }
\end{aligned}
$$

Corollary 6. Given a L.T. $\xi=T E^{\prime}$ relative to coordinates $\xi, \xi^{\prime}$, the transformation relative to "standard" coordinates $\xi=S \xi, \quad \xi^{\prime}=S^{\prime} \xi^{\prime}$ has the form

$$
\xi=\left(d / \Upsilon_{0}\right) L_{0} \xi^{\prime}
$$


$-255-$

Note 1. The Gram-Schmidt process affords a construction of a rotation $R$ which takes $\delta_{1}$ into a given unit vector $\Psi_{1}$. Since $\Psi_{1} \neq 0$, we may suppose its $i-t h$ component $a_{i} \neq 0$. The set of $n$ vectors comprised of $\Psi_{1}$ and all $\delta_{j}$ with $j \neq i$ is linearly independent. The orthonormalization algorithm produces from these an orthonormal set

$$
R=\left[\Psi_{1}, \Psi_{2}, \ldots, \Psi_{n}\right]
$$

of column vectors. Regarded as a matrix $R$ we have

$$
R^{\tau} R=I_{n} \text { and } R \delta_{1}=\Psi_{1}
$$


TABLE I

SOME PHYSICAL CONSTANTS

\begin{tabular}{|c|c|c|c|c|}
\hline & 2.9979 & $\times 10^{10}$ & $\mathrm{~cm} \mathrm{sec}-1$ & speed limit \\
\hline & 6.6262 & $\times 10^{-27}$ & erg sec & Planck's constant \\
\hline & 4.8033 & $\times 10^{-10}$ & esu chg & charge quantum \\
\hline$(\varepsilon)$ & 9.1096 & $\times 10^{-28}$ & $g m$ & $\varepsilon$ rest mass \\
\hline$(\varepsilon)$ & .51100 & & $\mathrm{MeV}$ & $\varepsilon$ rest energy $\mathrm{m}(\varepsilon) \mathrm{c}^{2} /\left(\mathrm{q} 10^{14} / \mathrm{c}\right)$ \\
\hline$(\varepsilon)$ & .024263 & $\times 10^{-8}$ & $\mathrm{~cm}$ & $\varepsilon$ Compton wavelength $h / m(\varepsilon) c$ \\
\hline$(\varepsilon)$ & 2.8179 & $\times 10^{-13}$ & $\mathrm{~cm}$ & $\varepsilon$ radius $q^{2} / m(\varepsilon) c^{2}$ \\
\hline & 1.0546 & $\times 10^{-27}$ & erg sec & unit ang. mom. $h / 2 \pi$ \\
\hline & .52918 & $\times 10^{-8}$ & $\mathrm{~cm}$ & 1st Bohr rad. $h^{2} / \mathrm{m}(\varepsilon) \mathrm{q}^{2}$ \\
\hline & 9.2741 & $\times 10^{-21}$ & erg/gauss & Bohr magneton $q^{h / 2 m(\varepsilon) c}$ \\
\hline & 6.0222 & $\times 10^{23}$ & & Avogadro's no. $\left({ }^{12} \mathrm{C}\right)$ \\
\hline & 1.3806 & $\times 10^{-16}$ & $\operatorname{erg} /\left({ }^{\circ} \mathrm{K}\right)$ & Boltzmann constant \\
\hline & 8.3143 & $\times 10^{7}$ & $\operatorname{erg} /\left({ }^{\circ} \mathrm{K}\right)$ & Gas constant $\mathrm{kN}_{\mathrm{O}}\left({ }^{12} \mathrm{C}\right)$ \\
\hline & 5.6696 & $\times 10^{-5}$ & $\begin{array}{r}\mathrm{erg} / \mathrm{cm}^{2} \mathrm{sec} \\
\left({ }^{0} \mathrm{~K}\right)^{4}\end{array}$ & $\begin{array}{l}\text { Stefan-Boltzmann constant } \\
(2 / 15) \pi^{5} \mathrm{c}(\mathrm{hc})^{-3} \kappa^{4}\end{array}$ \\
\hline
\end{tabular}

UNITS

\begin{tabular}{|c|c|c|c|}
\hline $\mathbf{f}$ & $10^{-13}$ & $\mathrm{~cm}$ & (fermi) \\
\hline$\AA$ & $10^{-8}$ & $\mathrm{~cm}$ & (\&ngstrom) \\
\hline $\mathbf{Y}$ & $3.16 \times 10^{7}$ & $\sec$ & (year) \\
\hline LY & $9.46 \times 10^{17}$ & $\mathrm{~cm}$ & (cY=light year) \\
\hline $\mathrm{Hz}$ & 1 & $\sec ^{-1}$ & (Hertz) \\
\hline MHz & $10^{6}$ & $\mathrm{~Hz}$ & (Mega-Hertz) \\
\hline eV & $1.6022 \times 10^{-}$ & -12 erg & $\left(\mathrm{q} 10^{8} / \mathrm{c}\right)$ \\
\hline C & $c / 10$ & esu & (Coulomb) \\
\hline v & $10^{8} / 0$ & volt & (Vo1t) \\
\hline & $10^{7}$ & erg & (Joule) \\
\hline
\end{tabular}


TABLE II

SOME ASTRONOMICAL CONSTANTS

\begin{tabular}{|c|c|c|}
\hline G & $6.670 \times 10^{-8} \mathrm{~cm}^{3} \mathrm{gm}^{-1} \mathrm{sec}^{-2}$ & gravitation constarit \\
\hline $\mathbf{g}$ & $980.665 \mathrm{~cm} / \mathrm{sec}^{2}$ & earth gravity constant $\mathrm{GM}_{E} / \mathrm{r}_{E}^{2}$ \\
\hline H & $3.24 \times 10^{-18} \mathrm{sec}^{-1}, 100(\mathrm{~km} / \mathrm{sec}) / \mathrm{Mpc}$ & Hubble's constant \\
\hline $\mathbf{S}$ & $.978 \times 10^{10} \mathrm{LY}, 3000 \mathrm{Mpc}$ & "radius of universe" $c / H$ \\
\hline $\mathrm{T}$ & $.978 \times 10^{10} \mathrm{Y}$ & time ago of "bang" $1 / \mathrm{H}$ \\
\hline$f$ & $1.99 \mathrm{cal} / \mathrm{min} \mathrm{cm}^{2}, 1.388 \times 10^{6} \mathrm{erg} / \mathrm{sec} \mathrm{cm}^{2}$ & sun's flux at earth \\
\hline$L_{\odot}$ & $3.9 \times 10^{33} \mathrm{erg} / \mathrm{sec}$ & sun's luminosity $4 \pi(A U)^{2} f$ \\
\hline$M_{\odot}$ & $1.989 \times 10^{33} \mathrm{gm}$ & sun's mass \\
\hline$R_{0}$ & $6.96 \times 10^{10} \mathrm{~cm}$ & sun's radius \\
\hline$\phi_{\odot}$ & $6.41 \times 10^{10} \mathrm{erg} / \mathrm{sec} \mathrm{cm}^{2}$ & sun's surface flux $f\left(A U / R_{\theta}\right)^{2}$ \\
\hline$T_{\odot}$ & $5800^{\circ} \mathrm{K}$ & sun's surface temp. $\phi_{\odot}=\sigma \mathrm{T}_{\odot}^{4}$ \\
\hline$L_{G}$ & $4 \times 10^{43} \mathrm{erg} / \mathrm{sec} \sim 10^{10} L_{\odot}$ & av. galaxy luminosity \\
\hline$M_{G}$ & -20.3 & av. galaxy abs. magnitude \\
\hline no & $10^{-75} \mathrm{~cm}^{-3}$ & no. galaxies $/ \mathrm{cm}^{3}$ \\
\hline $\mathbf{Y}$ & $.316 \times 10^{8} \mathrm{sec}$ & year \\
\hline LY & $.946 \times 10^{18} \mathrm{~cm}$ & light year $=c Y$ \\
\hline $\mathbf{A U}$ & $1.496 \times 10^{13} \mathrm{~cm}$ & astron. unit $=$ radius earth orbit \\
\hline pc & $3.086 \times 10^{18} \mathrm{~cm}, 3.262 \mathrm{LY}$ & $\mathrm{AU} /(1 / 3600)(\pi / 180)$, parsec \\
\hline Mpc & $10^{6} \mathrm{pc}$ & Megaparsec \\
\hline$\Phi_{0}$ & $2.4 \times 10^{44} \mathrm{erg} / \mathrm{sec}(\mathrm{Mpc})^{2}$ & Standard flux \\
\hline cal & $4.185 \times 10^{7} \mathrm{erg}$ & calorie \\
\hline
\end{tabular}


TABLE III

SOME "FUNDAMENTAL" PARTICLES

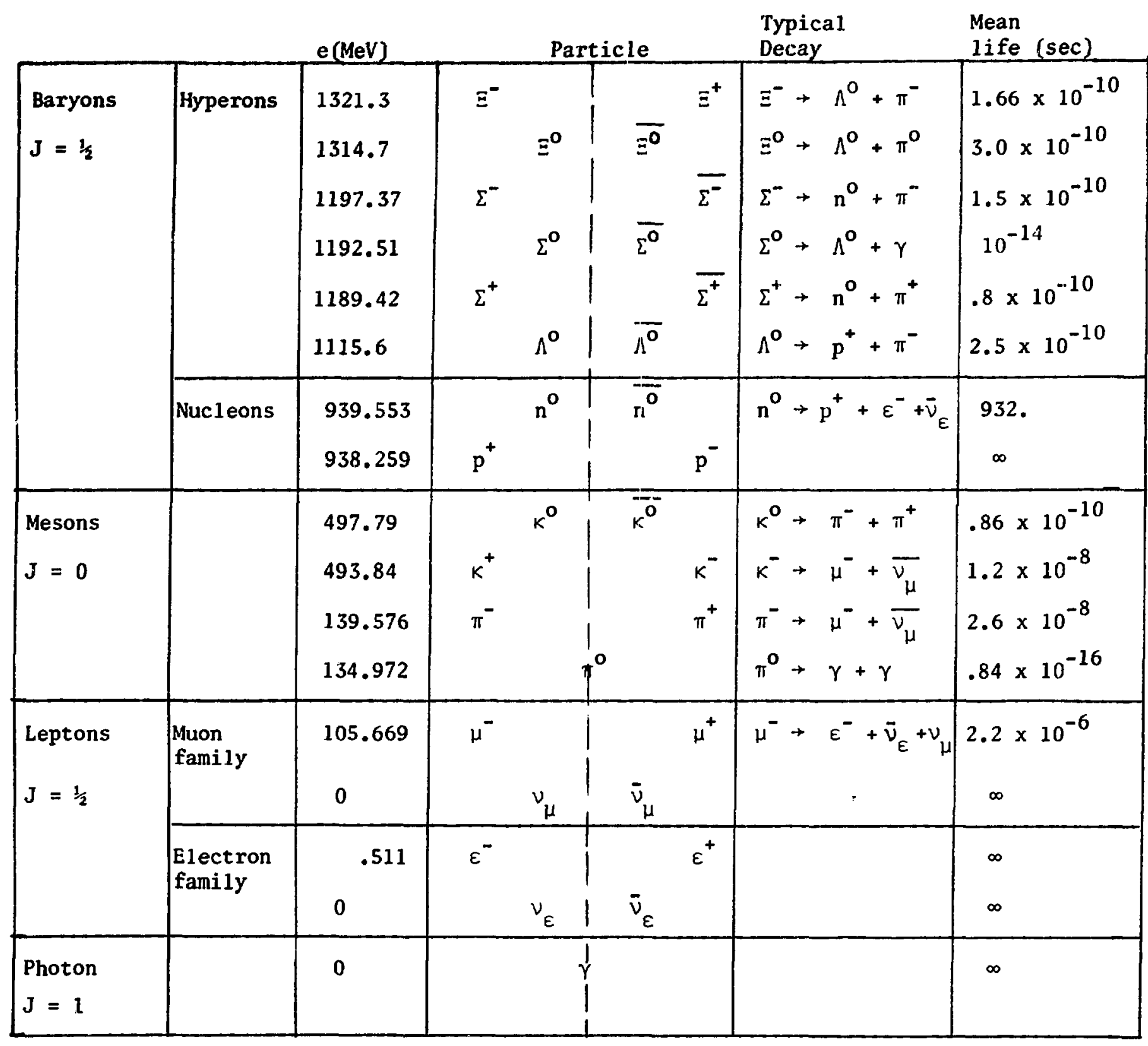


TABLE IV

SOME NEUTRAL ATOM REST MASSES

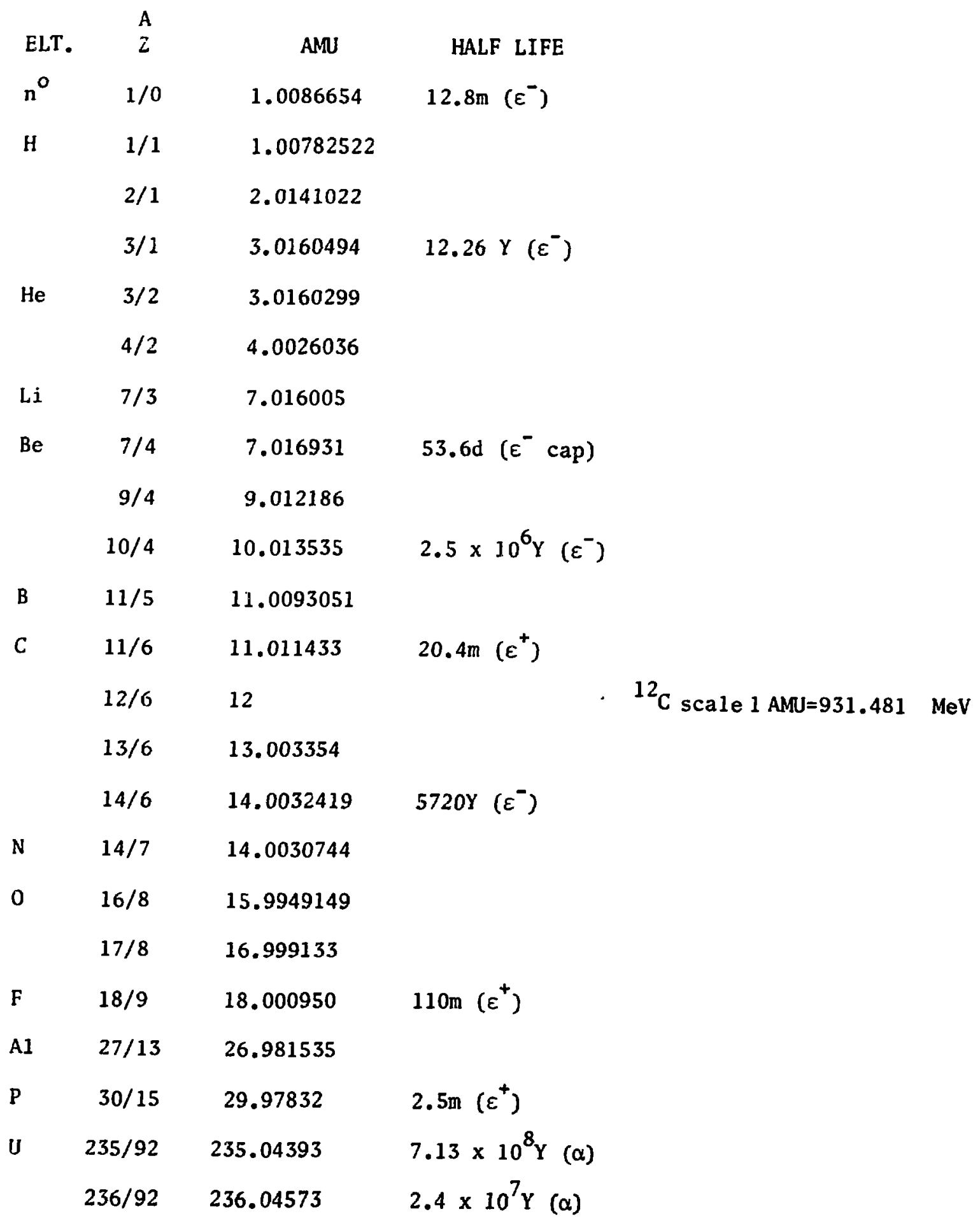




\section{TABLE V}

\section{SOME FORMULAS}

1. $\Gamma(s)=\int_{0}^{\infty} x^{s-1} e^{-x} d x=2 \int_{0}^{\infty} x^{2 s-1} e^{-x^{2}} d x, s>0$

$$
\Gamma\left(\frac{1}{2}\right)=\sqrt{ }, \quad \Gamma(n+1)=n \Gamma(n), n>0
$$

$$
\Gamma(n+1)=n !, \quad n=0,1,2, \ldots, \quad 0 ! \equiv 1
$$

2. $\zeta(s)=\sum_{\mathrm{m}=1}^{\infty} 1 / \mathrm{m}^{s}, \Delta>1$

$$
\zeta(2)=\pi^{2} / 6, \quad \zeta(3) \stackrel{\simeq}{=} 1.2021, \quad \zeta(4)=\pi^{4} / 90
$$

3. $A \cdot B=a_{x} b_{x}+a_{y} b_{y}+a_{z} b_{z}$

$$
|A|=(A \cdot A)^{\frac{1}{2}}=\left(a_{x}^{2}+a_{y}^{2}+a_{z}^{2}\right)^{\frac{1}{2}}, \quad \cos (A, B)=A \cdot B /|A| \cdot|B|
$$

4. A $\times B=\left\{\begin{array}{ccc}a_{x} & a_{y} & a_{z} \\ b_{x} & b_{y} & b_{z}\end{array}\right\}=\left[a_{y} b_{z}-a_{z} b_{y},-\left(a_{x} b_{z}-a_{z} b_{x}\right), a_{x} b_{y}-a_{y} b_{x}\right]$

$$
|A x B|=|A| \cdot|B| \sin (A, B)=|A||B|\left\{1-\cos ^{2}(A, B)\right\}^{\frac{1}{2}}
$$

5. $A \cdot(B \times C)=C \cdot(A \times B)=B \cdot(C \times A)$

6. $A \times(B \times C)=-(A \cdot B) C+(A \cdot C) B$ 